\title{
A Canonical Decomposition in Collective and Relative Variables of a Klein-Gordon Field in the Rest-Frame Wigner-Covariant Instant Form.
}

\author{
Luca Lusanna \\ Sezione INFN di Firenze \\ L.go E.Fermi 2 (Arcetri) \\ 50125 Firenze, Italy \\ E-mail:LUSANNA@FI.INFN.IT \\ and \\ Massimo Materassi \\ Dipartimento di Fisica \\ Universita' di Perugia \\ Via Elce di Sotto, \\ 06100 Perugia, Italy \\ E-mail: MATERASSI@PG.INFN.IT \\ MATERASSI@FI.INFN.IT
}

\begin{abstract}
The canonical decomposition of a real Klein-Gordon field in collective and relative variables proposed by Longhi and Materassi is reformulated on spacelike hypersurfaces. This allows to obtain the complete canonical reduction of the system on Wigner hyperplanes, namely in the rest-frame Wignercovariant instant form of dynamics. From the study of Dixon's multipoles for the energy-momentum tensor on the Wigner hyperplanes we derive the definition of the canonical center-of-mass variable for a Klein-Gordon field configuration: it turns out that the Longhi-Materassi global variable should be interpreted as a center of phase of the field configuration. A detailed study of the kinematical "external" and "internal" properties of the field configuration on the Wigner hyperplanes is done. The construction is then extended to charged Klein-Gordon fields: the centers of phase of the two real components can be combined to define a global center of phase and a collective relative variable describing the action-reaction between the two Feshbach-Villars components of the field with definite sign of energy and charge. The Dixon multipoles for both the energy-momentum and the electromagnetic current are given. Also the coupling of the Klein-Gordon field to scalar relativistic particles is studied and it is shown that in the reduced phase space, besides
\end{abstract}


the particle and field relative variables, there is also a collective relative variable describing the relative motion of the particle subsytem with respect to the field one.

Typeset using REVTEX 


\section{INTRODUCTION}

Recently a new instant form of dynamics, the rest-frame Wigner-covariant instant form, was defined in Ref. [1] as an important tool for the program of obtaining a unified description of the four interactions with a canonical reduction to the independent degrees of freedom (Dirac's observables for a generalized Coulomb gauge; see Ref. [2] for a review). This tool emerged from the study of the reformulation of ordinary theories in Minkowski spacetime on arbitrary spacelike hypersurfaces as a prerequisite to their description in curved spacetimes. Since this formulation is the classical background of the Tomonaga-Schwinger description of quantum field theory, this new instant form will also open the possibility to obtain a Wigner-covariant equal time quantization of Minkowski theories, to define a set of Tomonaga-Schwinger asymptotic states and to open new possibilities for the description of relativistic bound states, whose formulation in the framework of the standard Fock approach is still problematic (see Ref. [3] and the spurious solutions of the Bethe-Salpeter equation [4]).

In the rest-frame instant form of every isolated system there is a $3+1$ splitting of Minkowski spacetime with a foliation whose leaves, labelled by a scalar time parameter $\tau$, are the spacelike Wigner hyperplanes $\Sigma_{W \tau}$ orthogonal to the four-momentum of the isolated system (assumed timelike). In an arbitrary Lorentz frame the points of the Wigner hyperplane $\Sigma_{W \tau}$ have coordinates $z^{\mu}(\tau, \vec{\sigma})=x_{s}^{\mu}(\tau)+\epsilon_{r}^{\mu}\left(u\left(p_{s}\right)\right) \sigma^{r}$ : the point $x_{s}^{\mu}(\tau)$ is an arbitrary origin for the 3-coordinates $\vec{\sigma}$ on $\Sigma_{W \tau}$ and $p_{s}^{\mu}$, its conjugate momentum, is orthogonal to $\Sigma_{W \tau}\left[\epsilon_{r}^{\mu}\left(u\left(p_{s}\right)\right)\right.$ are three suitable spacelike four-vectors tangent to $\left.\Sigma_{W \tau}\right]$. After the canonical reduction of the isolated system to the Wigner hyperplanes we remain with four universal first class constraints [1]:

i) one, $p_{s}^{2} \approx M_{\text {sys }}^{2}$, identifying the invariant mass $M_{s y s}$ of the isolated system as the Hamiltonian for the evolution in the rest-frame time;

ii) three others defining the rest frame by the requirement that the (Wigner spin 1) total 3 -momentum of the isolated system is zero, $\vec{P}_{s y s} \approx 0$ [so that $p_{s}^{\mu} \approx M_{s y s} u^{\mu}\left(p_{s}\right)$, with the unit four-vector $U^{\mu}\left(p_{s}\right)$ describing the orientation of the Wigner hyperplanes with respect to the arbitrary Lorentz frame].

Among the physical canonical degrees of freedom there are the coordinates of a point of the Wigner hyperplane, different from its covariant origin $x_{s}^{\mu}(\tau)$ of the internal 3-coordinates, with canonical noncovariant coordinates $\tilde{x}_{s}^{\mu}(\tau)$. This point, the classical analogue of the Newton-Wigner position operator, is decoupled from the system, describes its "external" center-of-mass variable and may be interpreted as a decoupled point particle observer with his clock measuring the rest-frame time. Instead, on the Wigner hyperplane there are only physical canonical relative degrees of freedom, whose identification requires the addition of

three gauge fixing constraints $\vec{X}_{\text {sys }} \approx 0$, so that the constraints $\vec{X}_{\text {sys }} \approx 0$ and $\vec{P}_{\text {sys }} \approx 0$ are second class and may be eliminated. These constraints $\vec{X}_{\text {sys }} \approx 0$ may be interpreted as the elimination of an "internal" center-of-mass-like variable of the system, which is the gauge variable conjugated to the first class constraints $\vec{P}_{\text {sys }} \approx 0$, so not to have a double counting of the center-of-mass degrees of freedom. The constraints $\vec{X}_{\text {sys }} \approx 0$ eliminate this "internal" center-of-mass-like variable from the physical degrees of freedom forcing it to coincide with the arbitrary origin $x_{s}^{\mu}(\tau)=z^{\mu}\left(\tau, \vec{\sigma}=\vec{X}_{\text {sys }}=0\right)$ of the Wigner hyperplane.

An open problem is the identification of these constraints $\vec{X}_{\text {sys }} \approx 0$ for the various isolated 
systems. In a future paper [5] it will be studied for a system of scalar particles. Instead, in this paper we will study this probem for the real Klein-Gordon field, as a particular aspect of the more general problem of finding canonical collective and relative variables for its configurations. In Refs. [6,7] Longhi and Materassi found a set of such variables in the standard Lorentz covariant approach. In this paper we shall reformulate their solution on the Wigner hyperplanes starting from the formulation of the Klein-Gordon field on arbitrary spacelike hypersurfaces in Minkowski spacetime [8]. Then, after having expressed the energymomentum tensor of the Klein-Gordon field on the Wigner hyperplanes, where it assumes a form similar to the energy-momentum tensor of a relativistic perfect fluid, we shall study Dixon's multipoles [9] of the field. This will open the way to the identification of the "internal" center of mass $\vec{X}_{\phi}=\vec{q}_{\phi}$ of the Klein-Gordon field and to the realization that the Longhi-Materassi collective variable is not the center of mass but a "center of phase" of the field configuration.

By considering a Klein-Gordon field configuration as a relativistic extended object, we can make a detailed study of the kinematical properties of the description of such an object on Wigner hyperplanes.

Then we shall apply the same approach to the system of $\mathrm{N}$ scalar particles interacting with a real Klein-Gordon field and to the system of a complex charged Klein-Gordon field plus the electromagnetic field. In the latter case its two components with definite sign of energy and charge (identified by means of the Feshbach-Villars formalism in absence of interaction) generate two centers of phase, so that, besides the global center of phase of the complex field, there is a second collective variable describing the global action-reaction between the two components. A similar collective variable exists also for the system of $\mathrm{N}$ particles interacting with a real Klein-Gordon field.

In Section II the real Klein-Gordon field is reformulated on spacelike hypersurfaces in Minkowski spacetime, following Ref. [8], and then restricted to Wigner hyperplanes.

In Section III its modulus-phase variables on the Wigner hyperplane are defined.

In Section IV there is the definition of the collective and relative canonical variables and the inverse canonical transformation to express the original real Klein-Gordon field in terms of them. It is shown that each constant energy surface of the Klein-Gordon field is the disjoint union of symplectic manifolds. Also a set of canonical multipoles is defined for a certain class of field configurations and there are some comments on the self-interactions of the field.

In Section $\mathrm{V}$ the energy-momentum tensor of a configuration of the real Klein-Gordon field is restricted to the Wigner hyperplanes and there is a study of Dixon's multipoles on it. This suggests a definition for the relativistic center of mass of the field configuration.

In Section VI, by using the group-theoretical methods of Ref. [10], there is a complete discussion of the concepts of "external" and "internal" (with respect to the Wigner hyperplane) centers of mass of the field configuration. The Longhi-Materassi collective variable is interpreted as a "center of phase" of the field configuration.

In Section VII the isolated system of $\mathrm{N}$ scalar particles interacting with a real KleinGordon field is studied to visualize its collective variables and their interpretation.

In Section VIII the previous analysis is extended to the charged Klein-Gordon field by defining the collective and relative canonical variables for its positive and negative frequency parts with the help of the Feshbach-Villars formalism [11 13]. The two collective variables 
can be combined to give the overall "center of phase" of the field configuration plus a collective relative variable. Then there is a study of the coupling to the electromagnetic field and of the Dixon multipoles.

In the Conclusions there are some comments about the utility of the multipolar expansions of the Klein-Gordon field in general relativity and about the problems existing for the quantization of the collective and relative variables.

In Appendix A there are some notations for spacelike hypersurfaces.

In Appendix $\mathrm{B}$ there is a review of Longhi-Materassi papers.

In Appendix $\mathrm{C}$ there is a review of the Feshbach-Villars formalism and the definition of the Fourier coefficients of the Klein-Gordon field corresponding to the solutions of the squareroot Klein-Gordon equation with both positive (or negative) energy and electric charge in the free case.

In Appendix D there is the expression of the invariant mass for the charged KleinGordon field plus the electromagnetic field on the Wigner hyperplanes in terms of the Fourier coefficients defined in Appendix C. 


\section{THE REAL KLEIN-GORDON FIELD ON SPACELIKE HYPERSURFACES.}

In Ref. [8] there is the description of scalar electrodynamics on spacelike hypersurfaces. In this Section we shall review this description restricting ourselves to a real Klein-Gordon field with a self-interaction $V(\phi)$ following the scheme of Ref. [1] [see Appendix A for the notations; $\dot{\phi}(\tau, \vec{\sigma})=\partial \phi(\tau, \vec{\sigma}) / \partial \tau]$. The action of a scalar Klein-Gordon field reads

$$
\begin{aligned}
S= & \int d \tau d^{3} \sigma N(\tau, \vec{\sigma}) \sqrt{\gamma(\tau, \vec{\sigma})} \\
& \frac{1}{2}\left[g^{\tau \tau} \dot{\phi}^{2}+2 g^{\tau \check{r}} \dot{\phi} \partial_{\check{r}} \phi+g^{\check{r} \check{s}} \partial_{\check{r}} \phi \partial_{\check{s}} \phi-m^{2} \phi^{2}-2 V(\phi)\right](\tau, \vec{\sigma})= \\
= & \int d \tau d^{3} \sigma \sqrt{\gamma(\tau, \vec{\sigma})} \frac{1}{2}\left[\frac{1}{N}\left[\partial_{\tau}-N^{\check{r}} \partial_{\check{r}}\right] \phi\left[\partial_{\tau}-N^{\check{s}} \partial_{\check{s}}\right] \phi+\right. \\
+ & \left.N\left[\gamma^{\check{r} \check{s}} \partial_{\check{r}} \phi \partial_{\check{s}} \phi-m^{2} \phi^{2}-2 V(\phi)\right]\right](\tau, \vec{\sigma}) .
\end{aligned}
$$

where the configuration variables are $z^{\mu}(\tau, \vec{\sigma}), \phi(\tau, \vec{\sigma})=\tilde{\phi}(z(\tau, \vec{\sigma}))$. The main difference between the standard theory and the one on spacelike hypersurfaces is that now the configuration variables are the fields $\phi(\tau, \vec{\sigma})=\tilde{\phi}(z(\tau, \vec{\sigma}))=(\tilde{\phi} \circ z)(\tau, \vec{\sigma})$, with $\tilde{\phi}(x)$ solution of the Klein-Gordon equation. These new fields $\phi=\tilde{\phi} \circ z$ contain the nonlocal information about the $3+1$ splitting of Minkowski spacetime $M^{4}$ with a foliation of spacelike hypersurfaces $\Sigma_{\tau}$, obtained through an embedding $R \times \Sigma \rightarrow M^{4},(\tau, \vec{\sigma}) \mapsto z^{\mu}(\tau, \vec{\sigma})[\Sigma$ is an abstract 3-surface diffeomorphic to $R^{3}$ ]. The fields $\phi(\tau, \vec{\sigma})$ have a built-in definition of equal time associated with the Lorentz-scalar time parameter $\tau$ which labels the leaves of the foliation.

Since $z_{\tau}^{\mu}=N l^{\mu}+N^{\check{r}} z_{\check{r}}^{\mu}$, with the lapse and shift functions $N, N^{\check{r}}$ functionals of $z^{\mu}(\tau, \vec{\sigma})$ through the metric $g_{A B}(\tau, \vec{\sigma})$, one has $\frac{\partial}{\partial z_{\tau}^{\mu}}=l_{\mu} \frac{\partial}{\partial N}+z_{\tilde{s} \mu} \gamma^{\check{r} \check{r}} \frac{\partial}{\partial N^{\tilde{r}}}$.

The canonical momenta are

$$
\begin{aligned}
\pi(\tau, \vec{\sigma})= & \frac{\partial L}{\partial \partial_{\tau} \phi(\tau, \vec{\sigma})}=\frac{\sqrt{\gamma}(\tau, \vec{\sigma})}{N(\tau, \vec{\sigma})}\left[\dot{\phi}-N^{\check{r}} \partial_{\check{r}} \phi\right](\tau, \vec{\sigma}), \\
& \Rightarrow \dot{\phi}(\tau, \vec{\sigma})=\left[\frac{N}{\sqrt{\gamma}} \pi+N^{\check{r}} \partial_{\check{r}} \phi\right](\tau, \vec{\sigma}), \\
\rho_{\mu}(\tau, \vec{\sigma})= & -\frac{\partial L}{\partial \partial_{\tau} z^{\mu}(\tau, \vec{\sigma})}= \\
= & l_{\mu}(\tau, \vec{\sigma})\left[\frac{\sqrt{\gamma}}{2}\left[\frac{1}{N^{2}}\left(\dot{\phi}-N^{\check{r}} \partial_{\check{r}} \phi\right)^{2}-\gamma^{\check{r} \check{s}} \partial_{\check{r}} \phi \partial_{\check{s}} \phi+m^{2} \phi^{2}+2 V(\phi)\right]\right](\tau, \vec{\sigma})+ \\
& +z_{\check{s} \mu}(\tau, \vec{\sigma}) \gamma^{\check{s} \check{r}}(\tau, \vec{\sigma})\left[\frac{\sqrt{\gamma}}{N} \partial_{\check{r}} \phi\left(\dot{\phi}-N^{\check{u}} \partial_{\check{u}} \phi\right)\right](\tau, \vec{\sigma}) .
\end{aligned}
$$

We have the following primary constraints

$$
\begin{aligned}
\mathcal{H}_{\mu}(\tau, \vec{\sigma})= & \rho_{\mu}(\tau, \vec{\sigma})- \\
& \quad-l_{\mu}(\tau, \vec{\sigma})\left[\frac{\pi^{2}}{2 \sqrt{\gamma}}-\frac{\sqrt{\gamma}}{2}\left[\gamma^{\check{r} \check{s}} \partial_{\check{r}} \phi \partial_{\check{s}} \phi-m^{2} \phi^{2}-2 V(\phi)\right]\right](\tau, \vec{\sigma})- \\
- & z_{\check{s} \mu}(\tau, \vec{\sigma}) \gamma^{\check{r} \check{s}}(\tau, \vec{\sigma})\left[\pi \partial_{\check{r}} \phi\right](\tau, \vec{\sigma}) \approx 0,
\end{aligned}
$$

and the following Dirac Hamiltonian $\left[\lambda^{\mu}(\tau, \vec{\sigma})\right.$ are Dirac multiplier $]$ 


$$
H_{D}=\int d^{3} \sigma \lambda^{\mu}(\tau, \vec{\sigma}) \mathcal{H}_{\mu}(\tau, \vec{\sigma})
$$

By using the Poisson brackets

$$
\begin{aligned}
\left\{z^{\mu}(\tau, \vec{\sigma}), \rho_{\nu}\left(\tau, \vec{\sigma}^{\prime}\right)\right\} & =\eta_{\nu}^{\mu} \delta^{3}\left(\vec{\sigma}-\vec{\sigma}^{\prime}\right) \\
\left\{\phi(\tau, \vec{\sigma}), \pi\left(\tau, \vec{\sigma}^{\prime}\right)\right\} & =\delta^{3}\left(\vec{\sigma}-\vec{\sigma}^{\prime}\right)
\end{aligned}
$$

one finds that the time constancy of the primary constraints does not imply the existence of new secondary constraints. The constraints are first class with the algebra $\left\{\mathcal{H}_{\mu}(\tau, \vec{\sigma}), \mathcal{H}_{\nu}(\tau, \vec{\sigma})\right\}=0$.

The conserved Poincare' generators are

$$
\begin{aligned}
p_{s}^{\mu}(\tau) & =\int \rho^{\mu}(\tau, \vec{\sigma}) d^{3} \sigma \\
J_{s}^{\mu \nu}(\tau) & =\int\left[z^{\mu}(\tau, \vec{\sigma}) \rho^{\nu}(\tau, \vec{\sigma})-z^{\nu}(\tau, \vec{\sigma}) \rho^{\mu}(\tau, \vec{\sigma})\right] d^{3} \sigma .
\end{aligned}
$$

Following Ref. [1], we can restrict ourselves to spacelike hyperplanes $z^{\mu}(\tau, \vec{\sigma})=x_{s}^{\mu}(\tau)+$ $b_{\check{r}}^{\mu}(\tau) \sigma^{\check{r}}$, where the normal $l^{\mu}=\epsilon_{\alpha \beta \gamma}^{\mu} b_{1}^{\alpha}(\tau) b_{2}^{\beta}(\tau) b_{3}^{\gamma}(\tau)$ is $\tau$-independent [with $b_{\tau}^{\mu}=l^{\mu} ; b_{\check{A}}^{\mu}(\tau)=$ $\left(b_{\tau}^{\mu}, b_{\check{r}}^{\mu}(\tau)\right)$ is an orthonormal tetrad]. Using the results of that paper we find that $\left\{x_{s}^{\mu}, p_{s}^{\nu}\right\}^{*}=$ $-\eta^{\mu \nu}, J_{s}^{\mu \nu}=x_{s}^{\mu} p_{s}^{\nu}-x_{s}^{\nu} p_{s}^{\mu}+S_{s}^{\mu \nu}$ and that the constraints are reduced to the following ten ones at the level of Dirac brackets $\left[\right.$ now $\left.\gamma^{\check{r} \check{s}}=-\delta^{\check{r} \check{s}}\right]$

$$
\begin{aligned}
\tilde{\mathcal{H}}^{\mu}(\tau)= & \int d^{3} \sigma \mathcal{H}^{\mu}(\tau, \vec{\sigma})= \\
= & p_{s}^{\mu}-b_{\check{A}}^{\mu}(\tau) \tilde{P}_{\phi}^{\check{A}}=p_{s}^{\mu}-l^{\mu} \tilde{P}_{\phi}^{\tau}-b_{\check{r}}^{\mu}(\tau) P_{\phi}^{\check{r}}= \\
= & p_{s}^{\mu}-l^{\mu} \frac{1}{2} \int d^{3} \sigma\left[\pi^{2}+(\vec{\partial} \phi)^{2}+m^{2} \phi^{2}+2 V(\phi)\right](\tau, \vec{\sigma})- \\
- & b_{\check{r}}^{\mu}(\tau) \int d^{3} \sigma\left[\pi \partial_{\check{r}} \phi\right](\tau, \vec{\sigma}) \approx 0 \\
\tilde{\mathcal{H}}^{\mu \nu}(\tau)= & b_{\check{r}}^{\mu}(\tau) \int d^{3} \sigma \sigma^{\check{r}} \mathcal{H}^{\nu}(\tau, \vec{\sigma})-b_{\check{r}}^{\nu}(\tau) \int d^{3} \sigma \sigma^{\check{r}} \mathcal{H}^{\mu}(\tau, \vec{\sigma})= \\
= & S_{s}^{\mu \nu}-\left(b_{\check{r}}^{\mu}(\tau) l^{\nu}-b_{\check{r}}^{\nu}(\tau) l^{\mu}\right) \\
& \frac{1}{2} \int d^{3} \sigma \sigma^{\check{r}}\left[\pi^{2}+(\vec{\partial} \phi)^{2}+m^{2} \phi^{2}+2 V(\phi)\right](\tau, \vec{\sigma})- \\
- & \left(b_{\check{r}}^{\mu}(\tau) b_{\check{s}}^{\nu}(\tau)-b_{\check{r}}^{\nu}(\tau) b_{\check{s}}^{\mu}(\tau)\right) \int d^{3} \sigma \sigma^{\check{r}}\left[\pi \partial^{\check{s}} \phi\right](\tau, \vec{\sigma})= \\
= & S_{s}^{\mu \nu}-b_{\check{r}}^{\mu}(\tau) b_{\check{s}}^{\nu}(\tau) S_{\phi}^{\check{r} \check{s}}-\left(b_{\check{r}}^{\mu}(\tau) l^{\nu}-b_{\check{r}}^{\nu}(\tau) l^{\mu}\right) S_{\phi}^{\check{r} \tau} \approx 0
\end{aligned}
$$

where $\tilde{P}_{\phi}^{\check{A}}=\left(\tilde{P}_{\phi}^{\tau} ; P_{\phi}^{\check{r}}\right)$ is the (Lorentz-scalar) 4-momentum of the field configuration and $S_{\phi}^{\check{r} \check{s}}=\left.J_{\phi}^{\check{r} \check{s}}\right|_{\vec{P}_{\phi}=0}, S_{\phi}^{\tau \check{r}}=\left.J_{\phi}^{\text {or }}\right|_{\vec{P}_{\phi}=0}$ its spin tensor [see Appendix B].

The configuration variables are reduced from $z^{\mu}(\tau, \vec{\sigma}), \phi(\tau, \vec{\sigma})$, to $x_{s}^{\mu}(\tau)$, to the six independent degrees of freedom hidden in the orthonormal tetrad $b_{\tilde{A}}^{\mu}(\tau)$, to $\phi(\tau, \vec{\sigma})$, and to the associated momenta: $p_{s}^{\mu}$ conjugate to $x_{s}^{\mu}$; six degrees of freedom hidden in $S_{s}^{\mu \nu}$ as the momenta conjugate to the variables hidden in the orthonormal tetrad $b_{\check{A}}^{\mu}$ [see Ref. [1] for the associated Hanson-Regge Dirac brackets for these degrees of freedom]; $\pi(\tau, \vec{\sigma})$ conjugate to $\phi(\tau, \vec{\sigma})$. 
If we select all the configurations of the system with timelike total momentum $\left[p_{s}^{2}>0\right]$, we can restrict ourselves to the special Wigner hyperplanes $\Sigma_{W \tau}$ orthogonal to $p_{s}^{\mu}$. The procedure for doing this canonical reduction implies [1] to boost at rest the variables $b_{\tilde{A}}^{\mu}$, $S_{s}^{\mu \nu}$, with the standard Wigner boost $L^{\mu}{ }_{\nu}\left(p_{s}, \stackrel{\circ}{p}_{s}\right)$ for timelike Poincaré orbits $\left[p_{s}^{\mu}=\left(\epsilon_{s}=\eta_{s} \sqrt{p_{s}^{2}} ; \overrightarrow{0}\right)\right.$; $\left.\eta_{s}=\operatorname{sign} p_{s}^{o}\right]$ and then to add the gauge-fixings $b_{\check{A}}^{\mu}-L_{\nu=\check{A}}^{\mu}\left(p_{s}, \stackrel{\circ}{p}_{s}\right)=b_{\check{A}}^{\mu}-\epsilon_{\check{A}}^{\mu}\left(u\left(p_{s}\right)\right) \approx 0$ $\left[u_{\tilde{H}}^{\mu}\left(p_{s}\right)=p_{s}^{\mu} / \epsilon_{s}\right]$. These gauge-fixings [only six of them are independent], together with $\tilde{\mathcal{H}}^{\mu \nu}(\tau) \approx 0$, form six pairs of second class constraints. The Dirac brackets with respect to these second class constraints [implying $\tilde{\mathcal{H}}^{\mu \nu}(\tau) \equiv 0$ ] admit the following canonical basis: $\phi(\tau, \vec{\sigma}), \pi(\tau, \vec{\sigma}), \tilde{x}_{s}^{\mu}(\tau)$ [it is not a 4 -vector, but has only the covariance of the little group of timelike Poincaré orbits like the Newton-Wigner position operator], $p_{s}^{\mu}$. As shown in Ref. [1], the indices $\check{A}=(\tau, \check{r})$ are replaced by $A=(\tau, r)$; all the quantities $B^{\tau}$ are Lorentz scalars, while the quantities $\vec{B}=\left\{B^{r}\right\}$ are spin-1 Wigner 3-vectors; instead $p_{s}^{\mu}$ is a Minkowski 4 -vector giving the orientation of the Wigner hyperplane with respect to a given Lorentz frame. We get $J_{s}^{\mu \nu}=\tilde{x}_{s}^{\mu} p_{s}^{\nu}-\tilde{x}_{s}^{\nu} p_{s}^{\mu}+\tilde{S}_{s}^{\mu \nu}$ with the spin tensor $\tilde{S}_{s}^{\mu \nu}$ given in Eqs.(59) of Ref. [1].

Therefore, the final effect of these gauge-fixings is a canonical reduction to a phase space spanned only by the variables $\tilde{x}_{s}^{\mu}(\tau), p_{s}^{\mu}, \phi(\tau, \vec{\sigma}), \pi(\tau, \vec{\sigma})$, with standard Dirac brackets. Instead of $\tilde{x}_{s}^{\mu}, p_{s}^{\mu}$, describing a decoupled observer, one can use the canonical variables [1]: $\epsilon_{s}, T_{s}=p_{s} \cdot \tilde{x}_{s} / \epsilon_{s}=p_{s} \cdot x_{s} / \epsilon_{s}, \vec{k}_{s}=\vec{p}_{s} / \epsilon_{s}, \vec{z}_{s}=\epsilon_{s}\left[\overrightarrow{\tilde{x}}_{s}-\frac{\vec{p}_{s}}{p_{s}^{o}} \tilde{x}_{s}^{o}\right]\left[\vec{z}_{s}\right.$ is the noncovariant 3-coordinate corresponding to the Newton-Wigner position operator].

The only surviving four constraints are [now $\vec{P}_{\phi}=\left\{P_{\phi}^{r}\right\}$ is a spin-1 Wigner 3-vector]

$$
\begin{aligned}
\mathcal{H}(\tau) & =\epsilon_{s}-\tilde{P}_{\phi}^{\tau}= \\
& =\epsilon_{s}-\frac{1}{2} \int d^{3} \sigma\left[\pi^{2}+(\vec{\partial} \phi)^{2}+m^{2} \phi^{2}+2 V(\phi)\right](\tau, \vec{\sigma}) \approx 0, \\
\overrightarrow{\mathcal{H}}_{p}(\tau) & =\vec{P}_{\phi}=\int d^{3} \sigma[\pi \vec{\partial} \phi](\tau, \vec{\sigma}) \approx 0 .
\end{aligned}
$$

where $\tilde{P}_{\phi}^{A}=\left(\tilde{P}_{\phi}^{\tau} ; \vec{P}_{\phi}\right), \tilde{P}_{\phi}^{\tau}=P_{\phi}^{\tau}+\int d^{3} \sigma V(\phi)(\tau, \vec{\sigma})$, is the 4-momentum of the field configuration. The Dirac Hamiltonian is now $H_{D}=\lambda(\tau) \mathcal{H}+\vec{\lambda}(\tau) \cdot \overrightarrow{\mathcal{H}}_{p}$.

By defining $\bar{S}_{s}^{A B}=\epsilon_{\mu}^{A}\left(u\left(p_{s}\right)\right) \epsilon_{\nu}^{B}\left(u\left(p_{s}\right)\right) S_{s}^{\mu \nu}$, we can showRef. [1] that on the Wigner hyperplanes we have $\left.\bar{S}_{s}^{A B} \equiv J_{\phi}^{A B}\right|_{P_{\phi}^{D}=0}=S_{\phi}^{A B}\left[J_{\phi}^{A B}\right.$ is the angular momentum of the field configuration] and $\tilde{S}^{i j}=\delta^{i r} \delta^{j s} \bar{S}_{s}^{r s} \equiv \delta^{i r} \delta^{j s} \bar{S}_{\phi}^{r s}, \tilde{S}_{s}^{o i}=-\delta^{i r} \bar{S}_{s}^{r s} \delta^{s j} p_{s}^{j} /\left(p_{s}^{o}+\epsilon_{s}\right) \equiv-\delta^{i r} \bar{S}_{\phi}^{r s} \delta^{s j} p_{s}^{j} /\left(p_{s}^{o}+\epsilon_{s}\right)$, so that $J_{s}^{\mu \nu}$ becomes independent from the boosts $S_{\phi}^{\tau r}$. Therefore, the generators of the realization of the Poincaré group in the rest-frame Wigner-covariant instant form of dynamics ("external" Poincaré algebra) are $p_{s}^{\mu}\left[\right.$ or $\left.\epsilon_{s}, \vec{k}_{s}\right]$ and the Lorentz generators

$$
\begin{aligned}
& J_{s}^{i j}=\tilde{x}_{s}^{i} p_{s}^{j}-\tilde{x}_{s}^{j} p_{s}^{i}+\delta^{i r} \delta^{j s} \bar{S}_{s}^{r s}, \\
& J_{s}^{o i}=\tilde{x}_{s}^{o} p_{s}^{i}-\tilde{x}_{s}^{i} p_{s}^{o}-\frac{\delta^{i r} \bar{S}_{s}^{r s} p_{s}^{s}}{p_{s}^{o}+\epsilon_{s}}, \\
& \bar{S}_{s}^{r s} \equiv S_{\phi}^{r s}=\left.J_{\phi}^{r s}\right|_{\vec{P}_{\phi}=0}=\left.\int d^{3} \sigma\left\{\sigma^{r}\left[\pi \partial^{s} \phi\right](\tau, \vec{\sigma})-(r \leftrightarrow s)\right\}\right|_{\vec{P}_{\phi}=0} .
\end{aligned}
$$

With the gauge fixing $\chi=T_{s}-\tau \approx 0$, we can eliminate the variables $\epsilon_{s}, T_{s}$, and find that the $\tau$-evolution (in the Lorentz scalar rest-frame time $T_{s} \equiv \tau$ ) is governed by the Hamiltonian 


$$
\begin{aligned}
& H_{R}=M_{\phi}-\vec{\lambda}(\tau) \cdot \overrightarrow{\mathcal{H}}_{p}(\tau), \\
& M_{\phi}=\tilde{P}_{\phi}^{\tau}=\frac{1}{2} \int d^{3} \sigma\left[\pi^{2}+(\vec{\partial} \phi)^{2}+m^{2} \phi^{2}+2 V(\phi)\right](\tau, \vec{\sigma}),
\end{aligned}
$$

where $M_{\phi}$ is the invariant mass of the field configuration [it replaces the non relativistic Hamiltonian $H_{r e l}$ appearing in the separation of the center-of-mass motion $\left.H=\frac{\vec{P}^{2}}{2 M}+H_{r e l}\right]$.

In the gauge $\vec{\lambda}(\tau)=0$, the Hamilton equations are $\left[\triangle=-\vec{\partial}^{2}\right]$

$$
\begin{aligned}
\partial_{\tau} \phi(\tau, \vec{\sigma}) \stackrel{\circ}{=} & \pi(\tau, \vec{\sigma}), \\
\partial_{\tau} \pi(\tau, \vec{\sigma}) \stackrel{\circ}{=} & {\left[-\triangle-m^{2}\right] \phi(\tau, \vec{\sigma})-\frac{\partial V(\phi)}{\partial \phi}(\tau, \vec{\sigma}), } \\
& \Rightarrow\left[\partial_{\tau}^{2}+\triangle+m^{2}\right] \phi(\tau, \vec{\sigma}) \stackrel{\circ}{=}-\frac{\partial V(\phi)}{\partial \phi}(\tau, \vec{\sigma}) .
\end{aligned}
$$

We got a description in which the noncovariant canonical "external" center-of-mass 3variables $\vec{z}_{s}, \vec{k}_{s}$, move freely and are decoupled from the Klein-Gordon field variables $\phi(\tau, \vec{\sigma})$, $\pi(\tau, \vec{\sigma})$, living on the Wigner hyperplane and restricted by $\vec{P}_{\phi} \approx 0$. To reduce the field variables only to relative degrees of freedom one has to find an "internal" collective centerof-mass-like variable $\vec{X}_{\phi}[\phi, \pi]$ conjugate to $\vec{P}_{\phi}$, such that the gauge fixings $\vec{X}_{\phi} \approx 0$ force the position of the field "internal" collective variable $\vec{\sigma}=\vec{X}_{\phi}$ to coincide with the origin $x_{s}^{\mu}(\tau)=z^{\mu}(\tau, \vec{\sigma}=0)$ of the Wigner hyperplane. In this way we get a decoupled point particle observer (the "external" center of mass $\tilde{x}_{s}^{\mu}$ of the isolated system) whose scalar time $T_{s} \equiv \tau$ labels the evolution of the relative field variables on the Wigner hyperplanes, defined by the field configuration itself, foliating Minkowski spacetime. 


\section{FOURIER TRANSFORM AND MODULUS-PHASE VARIABLES ON THE WIGNER HYPERPLANE.}

In the rest-frame Wigner-covariant instant form on Wigner hyperplanes [in which one considers only field configurations with a timelike 4-momentum], we can define the Fourier coefficients of the fields $\phi(\tau, \vec{\sigma}), \pi(\tau, \vec{\sigma})$

$$
\left\{\begin{array}{l}
a(\tau, \vec{q})=\int d^{3} \sigma[\omega(q) \phi(\tau, \vec{\sigma})+i \pi(\tau, \vec{\sigma})] e^{i(\omega(q) \tau-\vec{q} \cdot \vec{\sigma})}, \\
a^{*}(\tau, \vec{q})=\int d^{3} \sigma[\omega(q) \phi(\tau, \vec{\sigma})-i \pi(\tau, \vec{\sigma})] e^{-i(\omega(q) \tau-\vec{q} \cdot \vec{\sigma})} \\
\phi(\tau, \vec{\sigma})=\int d \tilde{q}\left[a(\tau, \vec{q}) e^{-i(\omega(q) \tau-\vec{q} \cdot \vec{\sigma})}+a^{*}(\tau, \vec{q}) e^{+i(\omega(q) \tau-\vec{q} \cdot \vec{\sigma})}\right] \\
\pi(\tau, \vec{\sigma})=-i \int d \tilde{q} \omega(q)\left[a(\tau, \vec{q}) e^{-i(\omega(q) \tau-\vec{q} \cdot \vec{\sigma})}-a^{*}(\tau, \vec{q}) e^{+i(\omega(\vec{q}) \tau-\vec{q} \cdot \vec{\sigma})}\right]
\end{array}\right.
$$

with

$$
\omega(q)=\sqrt{m^{2}+\vec{q}^{2}}, d \tilde{q}=\frac{d^{3} q}{\Omega(q)}, \Omega(q)=(2 \pi)^{3} 2 \omega(q), \quad q=|\vec{q}|=\sqrt{\vec{q}^{2}} .
$$

Here $q_{A} \sigma^{A}=\omega(q) \tau-\vec{q} \cdot \vec{\sigma}$ is defined by using $q^{A}=\left(q^{\tau}=\omega(q) ; q^{r}\right)$ with $q^{\tau}$ Lorentz scalar and $\vec{q}$ a spin-1 Wigner 3-vector like $\vec{\sigma}$ [on arbitrary hyperplanes all the quantities $\tau, \vec{\sigma}, q^{\tau}$, $\vec{q}$, would be Lorentz scalars]. The Fourier coefficients are $\tau$-dependent only when there is a non null self interaction $V(\phi)$; they and their gradients are assumed to belong to the space $L_{2}(d \tilde{q})$.

From the Poisson brackets $\left\{\phi(\tau, \vec{\sigma}), \pi\left(\tau, \vec{\sigma}^{\prime}\right)\right\}=\delta^{3}\left(\vec{\sigma}-\vec{\sigma}^{\prime}\right)$, we get [leaving the factor $\Omega(q)$ to agree with the standard notations]

$$
\left\{a(\tau, \vec{q}), a^{*}(\tau, \vec{k})\right\}=-i \Omega(q) \delta^{3}(\vec{q}-\vec{k})
$$

The 4-momentum and angular momentum of the field configuration are $\left[\mathcal{V}\left[a, a^{*}\right]\right.$ being the contribution of the potential $V(\phi)$ when present]

$$
\begin{gathered}
\tilde{P}_{\phi}^{\tau}=P_{\phi}^{\tau}+\mathcal{V}\left[a, a^{*}\right]=\frac{1}{2} \int d^{3} \sigma\left[\pi^{2}+(\vec{\partial} \phi)^{2}+m^{2} \phi^{2}+2 V(\phi)\right](\tau, \vec{\sigma})= \\
=\int d \tilde{q} \omega(q) a^{*}(\tau, \vec{q}) a(\tau, \vec{q})+\mathcal{V}\left[a, a^{*}\right] \\
\vec{P}_{\phi}=\int d^{3} \sigma[\pi \vec{\partial} \phi](\tau, \vec{\sigma})=\int d \tilde{q} \vec{q} a^{*}(\tau, \vec{q}) a(\tau, \vec{q}) \\
J_{\phi}^{r s}=\int d^{3} \sigma\left[\pi\left(\sigma^{r} \partial^{s}-\sigma^{s} \partial^{r}\right) \phi\right](\tau, \vec{\sigma})= \\
=-i \int d \tilde{q} a^{*}(\tau, \vec{q})\left(q^{r} \frac{\partial}{\partial q^{s}}-q^{s} \frac{\partial}{\partial q^{r}}\right) a(\tau, \vec{q}) \\
J_{\phi}^{\tau r}=-\tau P_{\phi}^{r}+\frac{1}{2} \int d^{3} \sigma \sigma^{r}\left[\pi^{2}+(\vec{\partial} \phi)^{2}+m^{2} \phi^{2}\right](\tau, \vec{\sigma})= \\
=-\tau P_{\phi}^{r}+i \int d \tilde{q} \omega(q) a^{*}(\tau, \vec{q}) \frac{\partial}{\partial q^{r}} a(\tau, \vec{q}) .
\end{gathered}
$$


We want to define four variables $X_{\phi}^{A}[\phi, \pi]=\left(X_{\phi}^{\tau} ; \vec{X}_{\phi}\right)$ canonically conjugated to $P_{\phi}^{A}[\phi, \pi]=\left(P_{\phi}^{\tau} ; \vec{P}_{\phi}\right)$. First of all we make a canonical transformation to modulus-phase canonical variables

$$
\begin{aligned}
& \left\{\begin{array}{l}
a(\tau, \vec{q})=\sqrt{I(\tau, \vec{q})} e^{[i \varphi(\tau, \vec{q}]]}, \\
a^{*}(\tau, \vec{q})=\sqrt{I(\tau, \vec{q})} e^{[-i \varphi(\tau, \vec{q})]}, \\
I(\tau, \vec{q})=a^{*}(\tau, \vec{q}) a(\tau, \vec{q}), \\
\varphi(\tau, \vec{q})=\frac{1}{2 i} \ln \left[\frac{a(\tau, \vec{q})}{a^{*}(\tau, \vec{q})}\right],
\end{array}\right. \\
& \left\{I(\tau, \vec{q}), \varphi\left(\tau, \vec{q}^{\prime}\right)\right\}=\Omega(q) \delta^{3}\left(\vec{q}-\vec{q}^{\prime}\right) .
\end{aligned}
$$

In terms of the original canonical variables $\phi, \pi$, we have

$$
\begin{aligned}
& I(\tau, \vec{q})=\int d^{3} \sigma \int d^{3} \sigma^{\prime} e^{i \vec{q} \cdot\left(\vec{\sigma}-\vec{\sigma}^{\prime}\right)}[\omega(q) \phi(\tau, \vec{\sigma})-i \pi(\tau, \vec{\sigma})]\left[\omega(q) \phi\left(\tau, \vec{\sigma}^{\prime}\right)+i \pi\left(\tau, \vec{\sigma}^{\prime}\right)\right], \\
& \varphi(\tau, \vec{q})=\frac{1}{2 i} \ln \left[\frac{\int d^{3} \sigma[\omega(q) \phi(\tau, \vec{\sigma})+i \pi(\tau, \vec{\sigma})] e^{i(\omega(q) \tau-\vec{q} \cdot \vec{\sigma})}}{\int d^{3} \sigma^{\prime}\left[\omega(q) \phi\left(\tau, \vec{\sigma}^{\prime}\right)-i \pi\left(\tau, \vec{\sigma}^{\prime}\right)\right] e^{-i\left(\omega(q) \tau-\vec{q} \cdot \vec{\sigma}^{\prime}\right)}}\right]= \\
& =\omega(q) \tau+\frac{1}{2 i} \ln \left[\frac{\int d^{3} \sigma[\omega(q) \phi(\tau, \vec{\sigma})+i \pi(\tau, \vec{\sigma})] e^{-i \vec{q} \cdot \vec{\sigma}}}{\int d^{3} \sigma^{\prime}\left[\omega(q) \phi\left(\tau, \vec{\sigma}^{\prime}\right)-i \pi\left(\tau, \vec{\sigma}^{\prime}\right)\right] e^{i \vec{q} \cdot \vec{\sigma}^{\prime}}}\right] . \\
& \left\{\begin{array}{l}
\phi(\tau, \vec{\sigma})=\int d \tilde{q} \sqrt{I(\tau, \vec{q})}\left[e^{i \varphi(\tau, \vec{q})-i(\omega(q) \tau-\vec{q} \cdot \vec{\sigma})}+e^{-i \varphi(\tau, \vec{q})+i(\omega(q) \tau-\vec{q} \cdot \vec{\sigma})}\right] \\
\pi(\tau, \vec{\sigma})=-i \int d \tilde{q} \omega(q) \sqrt{I(\tau, \vec{q})}\left[e^{i \varphi(\tau, \vec{q})-i(\omega(q) \tau-\vec{q} \cdot \vec{\sigma})}-e^{-i \varphi(\tau, \vec{q})+i(\omega(q) \tau-\vec{q} \cdot \vec{\sigma})}\right] .
\end{array}\right.
\end{aligned}
$$

The Poincaré charges of the field configuration take the form

$$
\begin{gathered}
\left\{\begin{array}{l}
\tilde{P}_{\phi}^{\tau}=P_{\phi}^{\tau}+\mathcal{V}[I, \varphi]=\int d \tilde{q} \omega(q) I(\tau, \vec{q})+\mathcal{V}[I, \varphi], \\
\vec{P}_{\phi}=\int d \tilde{q} \vec{q} I(\tau, \vec{q}),
\end{array}\right. \\
\left\{\begin{array}{l}
J_{\phi}^{r s}=\int d \tilde{q} I(\tau, \vec{q})\left(q^{r} \frac{\partial}{\partial q^{s}}-q^{s} \frac{\partial}{\partial q^{r}}\right) \varphi(\tau, \vec{q}), \\
J_{\phi}^{\tau r}=-\tau P_{\phi}^{r}-\int d \tilde{q} \omega(q) I(\tau, \vec{q}) \frac{\partial}{\partial q^{r}} \varphi(\tau, \vec{q}) .
\end{array}\right.
\end{gathered}
$$

The classical analogue of the occupation number is $\left[\triangle=-\vec{\partial}^{2}\right]$

$$
\begin{aligned}
N_{\phi} & =\int d \tilde{q} a^{*}(\tau, \vec{q}) a(\tau, \vec{q})=\int d \tilde{q} I(\tau, \vec{q})= \\
& =\frac{1}{2} \int d^{3} \sigma\left[\pi \frac{1}{\sqrt{m^{2}+\triangle}}+\phi \sqrt{m^{2}+\triangle} \phi\right](\tau, \vec{\sigma}) .
\end{aligned}
$$




\section{COLLECTIVE AND RELATIVE CANONICAL VARIABLES.}

A sequence of canonical transformations from the field variables $\phi(x), \pi(x)=\frac{\partial \phi(x)}{\partial x^{o}}$ to the Fourier coefficients $a(\vec{k}), a^{*}(\vec{k})\left[k^{\mu}=\left(k^{o}=\omega(k=|\vec{k}|)=\sqrt{m^{2}+\vec{k}^{2}} ; \vec{k}\right)\right]$, then to the modulus-phase (or action-angle) variables $I(\vec{k}), \varphi(\vec{k})$, and finally to a canonical basis $X_{\phi}^{\mu}[F]$, $P_{\phi}^{\mu}, \mathcal{H}(\vec{k} \mid F], \mathcal{K}(\vec{k} \mid F]$ [depending on an arbitrary normalized weight function $F\left(P_{\phi}, k\right)$ ] was found in Ref. [6] when the real Klein-Gordon field $\phi(x)$ satisfies certain conditions. The main results of that paper are reviewed in Appendix B.

Here, we shall reformulate this approach on the Wigner hyperplane. Due to its peculiar Wigner covariance properties [1], the weight function $F\left(P_{\phi}, k\right)$ [with its generic non polynomial (for instance $F \approx e^{-P_{\phi} \cdot k}$ ) dependence upon $P_{\phi}^{\mu}$ due to manifest Lorentz covariance] may now be replaced by two separate scalar functions $\tilde{F}^{\tau}\left(P_{\phi}^{\tau}, q\right), \tilde{F}\left(\vec{P}_{\phi}, \vec{q}\right)$. Moreover, if we accept that these two functions are singular at $\vec{q}=0$, we can take them linear in $P_{\phi}^{\tau}$ and $\vec{P}_{\phi}$ respectively: $\tilde{F}^{\tau}\left(P_{\phi}^{\tau}, q\right)=P_{\phi}^{\tau} F^{\tau}(q), \tilde{F}\left(\vec{P}_{\phi}, \vec{q}\right)=-\vec{P}_{\phi} \cdot \vec{q} F(q)$ with a suitable normalization for $F^{\tau}(q)$ and $F(q)$.

From Appendix B, we see that we must require the following behaviours also on the Wigner hyperplane

$$
\begin{aligned}
& \text { i) } q \rightarrow \infty, \quad \sigma>0 \text {, } \\
& |a(\tau, \vec{q})| \rightarrow q^{-\frac{3}{2}-\sigma}, \quad|I(\tau, \vec{q})| \rightarrow q^{-3-\sigma}, \quad|\varphi(\tau, \vec{q})| \rightarrow q, \\
& \text { ii) } q \rightarrow 0, \quad \epsilon>0, \quad \eta>-\epsilon \text {, } \\
& |a(\tau, \vec{q})| \rightarrow q^{-\frac{3}{2}+\epsilon}, \quad|I(\tau, \vec{q})| \rightarrow q^{-3+\epsilon}, \quad|\varphi(\tau, \vec{q})| \rightarrow q^{\eta} .
\end{aligned}
$$

in order to have the Poincaré generators and the occupation number of Section III finite.

Let us remark that the collective and relative canonical variables can be defined in closed form only in absence of self-interactions of the field, so that in this Section we shall put $V(\phi)=\mathcal{V}[I, \varphi]=0$.

\section{A. Definition of the collective variables.}

Let us define the four functionals of the phases

$$
\begin{aligned}
& X_{\phi}^{\tau}=\int d \tilde{q} \omega(q) F^{\tau}(q) \varphi(\tau, \vec{q}), \\
& \vec{X}_{\phi}=\int d \tilde{q} \vec{q} F(q) \varphi(\tau, \vec{q}), \\
& \Rightarrow\left\{X_{\phi}^{r}, X_{\phi}^{s}\right\}=0, \quad\left\{X_{\phi}^{\tau}, X_{\phi}^{r}\right\}=0 .
\end{aligned}
$$

depending on two Lorentz scalar functions $F^{\tau}(q), F(q)$, whose form will be restricted by the following requirements implying that $X_{\phi}^{A}$ and $P_{\phi}^{A}$ are canonical variables:

$$
\left\{P_{\phi}^{\tau}, X_{\phi}^{\tau}\right\}=1,\left\{P_{\phi}^{r}, X_{\phi}^{s}\right\}=-\delta^{r s},\left\{P_{\phi}^{r}, X_{\phi}^{\tau}\right\}=0,\left\{P_{\phi}^{\tau}, X_{\phi}^{r}\right\}=0,
$$

Since $\left\{P_{\phi}^{\tau}, X_{\phi}^{\tau}\right\}=\int d \tilde{q} \omega^{2}(q) F^{\tau}(q)$ and $\left\{P_{\phi}^{r}, X_{\phi}^{s}\right\}=\int d \tilde{q} q^{r} q^{s} F(q)$, we must require the following normalizations for $F^{\tau}(q), F(q)$ 


$$
\begin{gathered}
\int d \tilde{q} \omega^{2}(q) F^{\tau}(q)=1 \\
\int d \tilde{q} q^{r} q^{s} F(q)=-\delta^{r s} .
\end{gathered}
$$

Moreover, $\left\{P_{\phi}^{r}, X_{\phi}^{\tau}\right\}=\int d \tilde{q} \omega(q) q^{r} F^{\tau}(q)$ and $\left\{P_{\phi}^{\tau}, X_{\phi}^{r}\right\}=\int d \tilde{q} \omega(q) q^{r} F(q)$, imply the following conditions

$$
\begin{gathered}
\int d \tilde{q} \omega(q) q^{r} F^{\tau}(q)=0, \\
\int d \tilde{q} \omega(q) q^{r} F(q)=0 .
\end{gathered}
$$

which are automatically satisfied because $F^{\tau}(q), F(q), q=|\vec{q}|$, are even under $q^{r} \rightarrow-q^{r}$.

A solution of Eqs.(4.4) and (4.5) is

$$
\begin{aligned}
F^{\tau}(q) & =\frac{16 \pi^{2}}{m q^{2} \sqrt{m^{2}+q^{2}}} e^{-\frac{4 \pi}{m^{2}} q^{2}}, \\
F(q) & =-\frac{48 \pi^{2}}{m q^{4}} \sqrt{m^{2}+q^{2}} e^{-\frac{4 \pi}{m^{2}} q^{2}} .
\end{aligned}
$$

The singularity in $\vec{q}=0$ requires $\varphi(\tau, \vec{q}) \rightarrow_{q \rightarrow 0} q^{\eta}, \eta>0$, [and not $\eta>-\epsilon$ as in Eq.(4.1)] for the existence of $X_{\phi}^{\tau}, \vec{X}_{\phi}$.

The analogue of the function $F\left(P_{\phi}, k\right)$ of Ref. [6] is now

$$
\begin{gathered}
\mathcal{F}\left(P_{\phi}, \vec{q}\right)=F^{\tau}(q) \omega(q) P_{\phi}^{\tau}-F(q) \vec{q} \cdot \vec{P}_{\phi}, \\
\\
\int d \tilde{q} \mathcal{F}\left(P_{\phi}, \vec{q}\right) \omega(q)=P_{\phi}^{\tau}, \\
\\
\int d \tilde{q} \mathcal{F}\left(P_{\phi}, \vec{q}\right) q^{r}=P_{\phi}^{r} .
\end{gathered}
$$

Let us remark that for field configurations $\phi(\tau, \vec{\sigma})$ such that the Fourier transform $\hat{\phi}(\tau, \vec{q})$ has compact support in a sphere centered at $\vec{q}=0$ of volume $\mathrm{V}$, we get $X_{\phi}^{\tau}=$ $-\frac{1}{V} \int \frac{d^{3} q}{\omega(q)} \varphi(\tau, \vec{q}), \vec{X}_{\phi}=\frac{1}{V} \int d^{3} q \frac{3 \vec{q}}{\vec{q}^{2}} \varphi(\tau, \vec{q})$.

\section{B. Auxiliary relative variables.}

As in Ref. [6], let us define an auxiliary relative action variable and an auxiliary relative phase variable

$$
\begin{gathered}
\hat{I}(\tau, \vec{q})=I(\tau, \vec{q})-F^{\tau}(q) P_{\phi}^{\tau} \omega(q)+F(q) \vec{q} \cdot \vec{P}_{\phi}, \\
\hat{\varphi}(\tau, \vec{q})=\varphi(\tau, \vec{q})-\omega(q) X_{\phi}^{\tau}+\vec{q} \cdot \vec{X}_{\phi} .
\end{gathered}
$$


The previous canonicity conditions on $F^{\tau}(q), F(q)$, imply

$$
\begin{gathered}
\int d \tilde{q} \omega(q) \hat{I}(\tau, \vec{q})=0 \\
\int d \tilde{q} q^{i} \hat{I}(\tau, \vec{q})=0 . \\
\int d \tilde{q} F^{\tau}(q) \omega(q) \hat{\varphi}(\tau, \vec{q}) d \tilde{q}=0 \\
\int d \tilde{q} F(q) q^{i} \hat{\varphi}(\tau, \vec{q})=0 .
\end{gathered}
$$

These auxiliary variables have the following nonzero Poisson bracket

$$
\{\hat{I}(\tau, \vec{k}), \hat{\varphi}(\tau, \vec{q})\}=\Delta(\vec{k}, \vec{q}),
$$

with

$$
\Delta(\vec{k}, \vec{q})=\Omega(k) \delta^{3}(\vec{k}-\vec{q})-F^{\tau}(k) \omega(k) \omega(q)+F(k) \vec{k} \cdot \vec{q} .
$$

The distribution $\Delta(\vec{k}, \vec{q})$ has the semigroup property

$$
\int d \tilde{q} \Delta(\vec{k}, \vec{q}) \Delta\left(\vec{q}, \vec{k}^{\prime}\right)=\Delta\left(\vec{k}, \vec{k}^{\prime}\right)
$$

and satisfies the constraints:

$$
\begin{gathered}
\int d \tilde{q} \omega(q) \Delta(\vec{q}, \vec{k})=0, \int d \tilde{q} q^{r} \Delta(\vec{q}, \vec{k})=0 \\
\int d \tilde{q} F^{\tau}(q) \omega(q) \Delta(\vec{k}, \vec{q})=0, \int d \tilde{q} q^{r} F(q) \Delta(\vec{k}, \vec{q})=0 .
\end{gathered}
$$

At this stage the canonical variables $I(\tau, \vec{q}), \varphi(\tau, \vec{q})$ for the Klein-Gordon field are replaced by the noncanonical set $X_{\phi}^{\tau}, P_{\phi}^{\tau}, \vec{X}_{\phi}, \vec{P}_{\phi}, \hat{I}(\tau, \vec{q}), \hat{\varphi}(\tau, \vec{q})$ with the Poisson brackets

$$
\left\{\begin{array}{l}
\left\{P_{\phi}^{\tau}, X_{\phi}^{\tau}\right\}=1,\left\{P_{\phi}^{r}, X_{\phi}^{s}\right\}=-\delta^{r s},\left\{P_{\phi}^{r}, X_{\phi}^{\tau}\right\}=0,\left\{P_{\phi}^{\tau}, X_{\phi}^{r}\right\}=0, \\
\left\{X_{\phi}^{r}, X_{\phi}^{s}\right\}=0,\left\{X_{\phi}^{\tau}, X_{\phi}^{r}\right\}=0,\left\{P_{\phi}^{A}, P_{\phi}^{B}\right\}=0, \quad A, B=(\tau, r), \\
\left\{P_{\phi}^{\tau}, \hat{I}(\tau, \vec{q})\right\}=0,\left\{P_{\phi}^{r}, \hat{I}(\tau, \vec{q})\right\}=0,\left\{\hat{I}(\tau, \vec{q}), X_{\phi}^{\tau}\right\}=0,\left\{\hat{I}(\tau, \vec{q}), X_{\phi}^{r}\right\}=0, \\
\left\{X_{\phi}^{r}, \hat{\varphi}(\tau, \vec{q})\right\}=0,\left\{X_{\phi}^{\tau}, \hat{\varphi}(\tau, \vec{q})\right\}=0,\left\{P_{\phi}^{r}, \hat{\varphi}(\tau, \vec{q})\right\}=0,\left\{P_{\phi}^{\tau}, \hat{\varphi}(\tau, \vec{q})\right\}=0, \\
\{\hat{I}(\tau, \vec{k}), \hat{\varphi}(\tau, \vec{q})\}=\Omega(k) \delta^{3}(\vec{k}-\vec{q})-F^{\tau}(k) \omega(k) \omega(q)+F(k) \vec{k} \cdot \vec{q} .
\end{array}\right.
$$

The generators of Lorentz group are already decomposed into two parts, the collective and the relative ones, each satisfying the Lorentz algebra and having vanishing mutual Poisson brackets 


$$
\begin{aligned}
& \left\{\begin{array}{l}
J_{\phi}^{r s}=L_{\phi}^{r s}+\hat{S}_{\phi}^{r s}, \\
L_{\phi}^{r s}=X_{\phi}^{r} P_{\phi}^{s}-X_{\phi}^{s} P_{\phi}^{r}, \\
\hat{S}_{\phi}^{r s}=\int d \tilde{q} \hat{I}(\tau, \vec{q})\left(q^{r} \frac{\partial}{\partial q^{s}}-q^{s} \frac{\partial}{\partial q^{r}}\right) \hat{\varphi}(\tau, \vec{q}),
\end{array}\right. \\
& \left\{\begin{array}{l}
J_{\phi}^{\tau r}=L_{\phi}^{\tau r}+\hat{S}_{\phi}^{\tau r}, \\
L_{\phi}^{\tau r}=\left[X_{\phi}^{\tau}-\tau\right] P_{\phi}^{r}-X_{\phi}^{r} P_{\phi}^{\tau}, \\
\hat{S}_{\phi}^{\tau r}=-\int d \tilde{q} \omega(q) \hat{I}(\tau, \vec{q}) \frac{\partial}{\partial q^{r}} \hat{\varphi}(\tau, \vec{q}) .
\end{array}\right.
\end{aligned}
$$

\section{Canonical relative variables.}

We must now find the canonical relative variables hidden inside the auxiliary ones, which are not free but satisfy Eqs.4.12) and (4.13).

As in Ref. [6], let us introduce the following differential operator $\left[\triangle_{L B}\right.$ is the LaplaceBeltrami operator of the mass shell submanifold $H_{3}^{1}$ (see Appendix B and Ref. [6,7])]

$$
\begin{aligned}
\mathcal{D}_{\vec{q}} & =3-m^{2} \triangle_{L B}= \\
& =3-m^{2}\left[\sum_{i=1}^{3}\left(\frac{\partial}{\partial q^{i}}\right)^{2}+\frac{2}{m^{2}} \sum_{i=1}^{3} q^{i} \frac{\partial}{\partial q^{i}}+\frac{1}{m^{2}}\left(\sum_{i=1}^{3} q^{i} \frac{\partial}{\partial q^{i}}\right)^{2}\right],
\end{aligned}
$$

which is a scalar on the Wigner hyperplane since it is invariant under Wigner's rotations.

Since $\omega$ (q) and $\vec{q}$ are null modes of this operator [6], we can put

$$
\begin{aligned}
\hat{I}(\tau, \vec{q}) & =\mathcal{D}_{\vec{q}} \mathbf{H}(\tau, \vec{q}), \\
\mathbf{H}(\tau, \vec{q}) & =\int d \tilde{k} \mathcal{G}(\vec{q}, \vec{k}) \hat{I}(\tau, \vec{k}),
\end{aligned}
$$

with $\mathcal{G}(\vec{q}, \vec{k})$ being the Green function of $\mathcal{D}_{\vec{q}}$ [see Refs. [6,7] for its expression]

$$
\mathcal{D}_{\vec{q}} \mathcal{G}(\vec{q}, \vec{k})=\Omega(k) \delta^{3}(\vec{k}-\vec{q}) .
$$

Like in Ref. [6], for each zero mode $f_{o}(\vec{q})$ of $\mathcal{D}_{\vec{q}}\left[\mathcal{D}_{\vec{q}} f_{o}(\vec{q})=0\right]$ for which one has $\left|\int d \tilde{q} f_{o}(\vec{q}) \hat{I}(\tau, \vec{q})\right|<\infty$, we have by integration by parts

$$
\begin{aligned}
\int d \tilde{q} \quad & f_{o}(\vec{q}) \hat{I}(\tau, \vec{q})=\int d \tilde{q} f_{o}(\vec{q}) \mathcal{D}_{\vec{q}} \mathbf{H}(\tau, \vec{q})= \\
= & -\frac{1}{2(2 \pi)^{3}} \int d^{3} q \frac{\partial}{\partial q^{r}}\left(\frac{m^{2} \delta^{r s}+q^{r} q^{s}}{\omega(q)}\left[f_{o}(\vec{q}) \frac{\partial}{\partial q^{s}} \mathbf{H}(\tau, \vec{q})-\mathbf{H}(\tau, \vec{q}) \frac{\partial}{\partial q^{s}} f_{o}(\vec{q})\right]\right) .
\end{aligned}
$$

The boundary conditions (ensuring finite Poincaré generators) 


$$
\begin{array}{ll}
\mathbf{H}(\tau, \vec{q}) \rightarrow_{q \rightarrow 0} q^{-1+\epsilon}, & \epsilon>0, \\
\mathbf{H}(\tau, \vec{q}) \rightarrow_{q \rightarrow \infty} q^{-3-\sigma}, & \sigma>0,
\end{array}
$$

imply $\int d \tilde{q} f_{o}(\vec{q}) \hat{I}(\tau, \vec{q})=0$, or

$$
\begin{aligned}
\int d \tilde{q} f_{o}(\vec{q}) I(\tau, \vec{q}) & =P_{\phi}^{\tau} \int d \tilde{q} \omega(q) f_{o}(\vec{q}) F^{\tau}(q)- \\
& -\vec{P}_{\phi} \cdot \int d \tilde{q} \vec{q} f_{o}(\vec{q}) F(q) .
\end{aligned}
$$

We shall restrict ourselves to field configurations for which $I(\tau, \vec{q}) \rightarrow_{q \rightarrow 0} q^{-3+\eta}$ with $\eta \in$ $(0,1]$ to avoid the extra condition connected with the zero modes $f_{o}(\vec{q})=v_{2,-3, l m}^{(o)}(\vec{q})$ [see the $Q_{l m}$ of Appendix B].

Instead, for the zero modes $f_{o}(\vec{q})=v_{1,-3, l m}^{(o)}(\vec{q})=q_{2}^{l} F_{1}\left(\frac{l-1}{2}, \frac{l+3}{2} ; l+\frac{3}{2} ;-q^{2}\right) Y_{l m}(\alpha, \beta)$, $[\vec{q}=q(\cos \alpha \cos \beta, \cos \alpha \sin \beta, \sin \alpha)]$ of Ref. [6] we get from Eq.(4.27): i) for $1=0,1$, the identities $P_{\phi}^{\tau}=P_{\phi}^{\tau}, \vec{P}_{\phi}=\vec{P}_{\phi}\left[v_{1,-3,00}^{(o)}\right.$ and $v_{1,-3,1 m}^{(o)}$ give $\left.f_{o}(\vec{q})=\omega(q), \vec{q}\right]$; ii) for $l \geq 2$ the conditions

$$
\begin{aligned}
P_{l m}= & \int d \tilde{q} v_{1,-3, l m}^{(o)}(\vec{q}) I(\tau, \vec{q})= \\
= & \text { const. } \int d \tilde{q} q_{2}^{l} F_{1}\left(\frac{l-1}{2}, \frac{l+3}{2} ; l+\frac{3}{2} ;-q^{2}\right) Y_{l m}(\theta, \varphi) \\
& \int d^{3} \sigma \int d^{3} \sigma^{\prime} e^{i \vec{q} \cdot\left(\vec{\sigma}-\vec{\sigma}^{\prime}\right)}[\omega(q) \phi(\tau, \vec{\sigma})-i \pi(\tau, \vec{\sigma})]\left[\omega(q) \phi\left(\tau, \vec{\sigma}^{\prime}\right)+i \pi\left(\tau, \vec{\sigma}^{\prime}\right)\right]=0 .
\end{aligned}
$$

Since $\vec{q} \cdot\left(\vec{\sigma}-\vec{\sigma}^{\prime}\right)=q \cos \theta\left|\vec{\sigma}-\vec{\sigma}^{\prime}\right|$, one can check that $P_{l m \neq 0}$ is automatically zero. Moreover, in $P_{l 0}$ the term $\omega(q)\left[\phi(\tau, \vec{\sigma}) \pi\left(\tau, \vec{\sigma}^{\prime}\right)-\phi\left(\tau, \vec{\sigma}^{\prime}\right) \pi(\tau, \vec{\sigma})\right]$ does not contribute for symmetry reasons. Therefore, the final restriction is

$$
\begin{aligned}
P_{l 0}= & \text { const. } \int d \tilde{q} q_{2}^{l} F_{1}\left(\frac{l-1}{2}, \frac{l+3}{2} ; l+\frac{3}{2} ;-q^{2}\right) Y_{l 0}(\theta, \varphi) \\
& \int d^{3} \sigma \int d^{3} \sigma^{\prime} e^{i \vec{q} \cdot\left(\vec{\sigma}-\vec{\sigma}^{\prime}\right)}\left[\left(m^{2}+q^{2}\right) \phi(\tau, \vec{\sigma}) \phi\left(\tau, \vec{\sigma}^{\prime}\right)+\pi(\tau, \vec{\sigma}) \pi\left(\tau, \vec{\sigma}^{\prime}\right)\right]=0 .
\end{aligned}
$$

These conditions on $\phi(\tau, \vec{\sigma})$ and $\pi(\tau, \vec{\sigma})=\partial_{\tau} \phi(\tau, \vec{\sigma})$ identify the class of configurations of the Klein-Gordon field for which one can define the previous canonical transformation and for which there is no ambiguity in defining a unique realization of the Poincaré group (the BMS algebra degenerates in the Poincaré algebra in this case).

We can satisfy the constraints on $\hat{\varphi}(\tau, \vec{q})$ with the definition $\left[\mathcal{D}_{\vec{q}} \omega(q)=\mathcal{D}_{\vec{q}} \vec{q}=0\right]$

$$
\begin{aligned}
\hat{\varphi}(\tau, \vec{q})= & \int d \tilde{k} \int d \tilde{k}^{\prime} \mathbf{K}(\tau, \vec{k}) \mathcal{G}\left(\vec{k}, \vec{k}^{\prime}\right) \Delta\left(\vec{k}^{\prime}, \vec{q}\right), \\
\mathbf{K}(\tau, \vec{q})= & \mathcal{D}_{\vec{q}} \hat{\varphi}(\tau, \vec{q})=\mathcal{D}_{\vec{q}} \varphi(\tau, \vec{q}), \\
& \rightarrow_{q \rightarrow \infty} q^{1-\epsilon}, \quad \epsilon>0, \quad \rightarrow_{q \rightarrow 0} q^{\eta-2}, \quad \eta>0,
\end{aligned}
$$

which also implies 


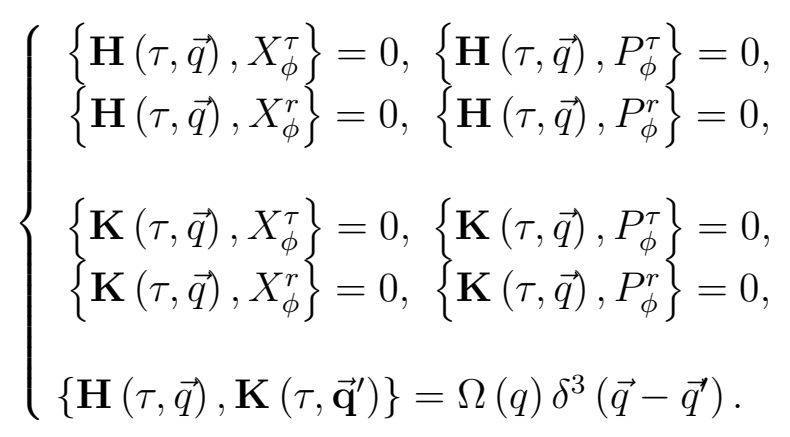

The final decomposition of Lorentz generators is

$$
\begin{gathered}
\left\{\begin{array}{l}
J_{\phi}^{r s}=L_{\phi}^{r s}+S_{\phi}^{r s}, \\
L_{\phi}^{r s}=X_{\phi}^{r} P_{\phi}^{s}-X_{\phi}^{s} P_{\phi}^{r}, \\
S_{\phi}^{r s}=\int d \tilde{k} \mathbf{H}(\tau, \vec{k})\left(k^{r} \frac{\partial}{\partial k^{s}}-k^{s} \frac{\partial}{\partial k^{r}}\right) \mathbf{K}(\tau, \vec{k}),
\end{array}\right) \\
\left\{\begin{array}{l}
J_{\phi}^{\tau r}=L_{\phi}^{\tau r}+S_{\phi}^{\tau r} \\
L_{\phi}^{\tau r}=\left(X_{\phi}^{\tau}-\tau\right) P_{\phi}^{r}-X_{\phi}^{r} P_{\phi}^{\tau} \\
S_{\phi}^{\tau r}=-\int d \tilde{q} \omega(q) \mathbf{H}(\tau, \vec{q}) \frac{\partial}{\partial q^{r}} \mathbf{K}(\tau, \vec{q}) .
\end{array}\right)
\end{gathered}
$$

\section{Field variables in terms of collective-relative variables.}

We have found the canonical transformation

$$
\begin{aligned}
I(\tau, \vec{q})= & F^{\tau}(q) \omega(q) P_{\phi}^{\tau}-F(q) \vec{q} \cdot \vec{P}_{\phi}+\mathcal{D}_{\vec{q}} \mathbf{H}(\tau, \vec{q}), \\
\varphi(\tau, \vec{q})= & \int d \tilde{k} \int d \tilde{k}^{\prime} \mathbf{K}(\tau, \vec{k}) \mathcal{G}\left(\vec{k}, \vec{k}^{\prime}\right) \Delta\left(\vec{k}^{\prime}, \vec{q}\right)+\omega(q) X_{\phi}^{\tau}-\vec{q} \cdot \vec{X}_{\phi}, \\
N_{\phi}= & P_{\phi}^{\tau} \int d \tilde{q} \omega(q) F^{\tau}(q)-\vec{P}_{\phi} \cdot \int d \tilde{q} \vec{q} F(q)+\int d \tilde{q} \mathcal{D}_{\vec{q}} \mathbf{H}(\tau, \vec{q})= \\
= & \tilde{c} \frac{P_{\phi}^{\tau}}{m}+\int d \tilde{q} \mathcal{D}_{\vec{q}} \mathbf{H}(\tau, \vec{q}), \\
& \tilde{c}=m \int d \tilde{q} \omega(q) F^{\tau}(q)=2 \int_{0}^{\infty} \frac{d q}{\sqrt{m^{2}+q^{2}}} e^{-\frac{4 \pi}{m^{2}} q^{2}}=2 e^{4 \pi} \int_{m}^{\infty} \frac{d x}{\sqrt{x^{2}-m^{2}}} e^{-\frac{4 \pi}{m^{2}} x^{2}},
\end{aligned}
$$

with the two functions $F^{\tau}(q), F(q)$ given in Eqs.(4.8). Its inverse is

$$
\begin{aligned}
P_{\phi}^{\tau} & =\int d \tilde{q} \omega(q) I(\tau, \vec{q})=\frac{1}{2} \int d^{3} \sigma\left[\pi^{2}+(\vec{\partial} \phi)^{2}+m^{2} \phi^{2}\right](\tau, \vec{\sigma}), \\
\vec{P}_{\phi} & =\int d \tilde{q} \vec{q} I(\tau, \vec{q})=\int d^{3} \sigma[\pi \vec{\partial} \phi](\tau, \vec{\sigma}),
\end{aligned}
$$




$$
\begin{aligned}
& X_{\phi}^{\tau}=\int d \tilde{q} \omega(q) F^{\tau}(q) \varphi(\tau, \vec{q})=\tau+ \\
& +\frac{1}{2 \pi i m} \int d^{3} q \frac{e^{-\frac{4 \pi}{m^{2}} q^{2}}}{q^{2} \omega(q)} \ln \left[\frac{\omega(q) \int d^{3} \sigma e^{i \vec{q} \cdot \vec{\sigma}} \phi(\tau, \vec{\sigma})+i \int d^{3} \sigma e^{i \vec{q} \cdot \vec{\sigma}} \pi(\tau, \vec{\sigma})}{\omega(q) \int d^{3} \sigma e^{-i \vec{q} \cdot \vec{\sigma}} \phi(\tau, \vec{\sigma})-i \int d^{3} \sigma e^{-i \vec{q} \cdot \vec{\sigma}} \pi(\tau, \vec{\sigma})}\right]= \\
& \stackrel{\text { def }}{=} \tau+\tilde{X}_{\phi}^{\tau}, \quad \Rightarrow \quad L_{\phi}^{\tau r}=\tilde{X}_{\phi}^{\tau} P_{\phi}^{r}-X_{\phi}^{r} P_{\phi}^{\tau} \text {, } \\
& \vec{X}_{\phi}=\int d \tilde{q} \vec{q} F(q) \varphi(\tau, \vec{q})= \\
& =\frac{2 i}{\pi m} \int d^{3} q \frac{q^{i}}{q^{4}} e^{-\frac{4 \pi}{m^{2}} q^{2}} \ln \left[\frac{\sqrt{m^{2}+q^{2}} \int d^{3} \sigma e^{i \vec{q} \cdot \vec{\sigma}} \phi(\tau, \vec{\sigma})+i \int d^{3} \sigma e^{i \vec{q} \cdot \vec{\sigma}} \pi(\tau, \vec{\sigma})}{\sqrt{m^{2}+q^{2}} \int d^{3} \sigma e^{-i \vec{q} \cdot \vec{\sigma}} \phi(\tau, \vec{\sigma})-i \int d^{3} \sigma e^{-i \vec{q} \cdot \vec{\sigma}} \pi(\tau, \vec{\sigma})}\right], \\
& \mathbf{H}(\tau, \vec{q})=\int d \tilde{k} \mathcal{G}(\vec{q}, \vec{k})\left[I(\tau, \vec{k})-F^{\tau}(k) \omega(k) \int d \tilde{q}_{1} \omega\left(q_{1}\right) I\left(\tau, \vec{q}_{1}\right)+\right. \\
& \left.+F(k) \vec{k} \cdot \int d \tilde{q}_{1} \vec{q}_{1} I\left(\tau, \vec{q}_{1}\right)\right]= \\
& =\int d^{3} \sigma_{1} d^{3} \sigma_{2}\left[\pi\left(\tau, \vec{\sigma}_{1}\right) \pi\left(\tau, \vec{\sigma}_{2}\right) \int d \tilde{k} \mathcal{G}(\vec{q}, \vec{k}) \int d \tilde{k}_{1} \triangle\left(\vec{k}, \vec{k}_{1}\right) e^{i \vec{k}_{1} \cdot\left(\vec{\sigma}_{1}-\vec{\sigma}_{2}\right)}+\right. \\
& +\phi\left(\tau, \vec{\sigma}_{1}\right) \phi\left(\tau, \vec{\sigma}_{2}\right) \int d \tilde{k} \mathcal{G}(\vec{q}, \vec{k}) \int d \tilde{k}_{1} \omega^{2}\left(k_{1}\right) \triangle\left(\vec{k}, \vec{k}_{1}\right) e^{i \vec{k}_{1} \cdot\left(\vec{\sigma}_{1}-\vec{\sigma}_{2}\right)}- \\
& -i\left(\pi\left(\tau, \vec{\sigma}_{1}\right) \phi\left(\tau, \vec{\sigma}_{2}\right)+\pi\left(\tau, \vec{\sigma}_{2}\right) \phi\left(\tau, \vec{\sigma}_{1}\right)\right) \\
& \left.\int d \tilde{k} \mathcal{G}(\vec{q}, \vec{k}) \int d \tilde{k}_{1} \omega\left(k_{1}\right) \triangle\left(\vec{k} \cdot \vec{k}_{1}\right) e^{i \vec{k}_{1} \cdot\left(\vec{\sigma}_{1}-\vec{\sigma}_{2}\right)}\right] \\
& \mathbf{K}(\tau, \vec{q})=\mathcal{D}_{\vec{q}} \hat{\varphi}(\tau, \vec{q})=\mathcal{D}_{\vec{q}} \varphi(\tau, \vec{q})= \\
& =\frac{1}{2 i} \mathcal{D}_{\vec{q}} \ln \left[\frac{\int d^{3} \sigma[\omega(q) \phi(\tau, \vec{\sigma})+i \pi(\tau, \vec{\sigma})] e^{-i \vec{q} \cdot \vec{\sigma}}}{\int d^{3} \sigma^{\prime}\left[\omega(q) \phi\left(\tau, \vec{\sigma}^{\prime}\right)-i \pi\left(\tau, \vec{\sigma}^{\prime}\right)\right] e^{i \vec{q} \cdot \vec{\sigma}^{\prime}}}\right]
\end{aligned}
$$

We get the following expression of the other canonical variables $a(\tau, \vec{q}), \phi(\tau, \vec{\sigma}), \pi(\tau, \vec{\sigma})$, in terms of the final ones

$$
\begin{aligned}
& a(\tau, \vec{q})=\sqrt{F^{\tau}(q) \omega(q) P_{\phi}^{\tau}-F(q) \vec{q} \cdot \vec{P}_{\phi}+\mathcal{D}_{\vec{q}} \mathbf{H}(\tau, \vec{q})} \\
& e^{i\left[\omega(q) X_{\phi}^{\tau}-\vec{q} \cdot \vec{X}_{\phi}\right]+i \int d \tilde{k} \int d \tilde{k}^{\prime} \mathbf{K}(\tau, \vec{k}) \mathcal{G}\left(\vec{k}, \vec{k}^{\prime}\right) \Delta\left(\vec{k}^{\prime}, \vec{q}\right)}, \\
& N_{\phi}=\tilde{c} \frac{P_{\phi}^{\tau}}{m}-+\int d \tilde{k} \mathcal{D}_{\vec{k}} \mathbf{H}(\tau, \vec{k}), \\
& \phi(\tau, \vec{\sigma})=\int d \tilde{q} \sqrt{F^{\tau}(q) \omega(q) P_{\phi}^{\tau}-F(q) \vec{q} \cdot \vec{P}_{\phi}+\mathcal{D}_{\vec{q}} \mathbf{H}(\tau, \vec{q})}
\end{aligned}
$$

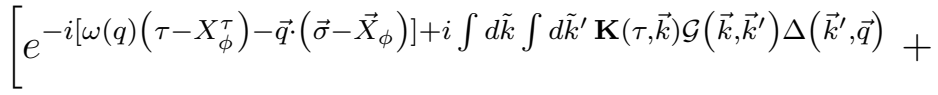

$$
\begin{aligned}
& +e^{\left.i\left[\omega(q)\left(\tau-X_{\phi}^{\tau}\right)-\vec{q} \cdot\left(\vec{\sigma}-\vec{X}_{\phi}\right)\right]-i \int d \tilde{k} \int d \tilde{k}^{\prime} \mathbf{K}(\tau, \vec{k}) \mathcal{G}\left(\vec{k}, \vec{k}^{\prime}\right) \Delta\left(\vec{k}^{\prime}, \vec{q}\right)\right]}= \\
& =2 \int d \tilde{q} \mathbf{A}_{\vec{q}}\left(\tau ; P_{\phi}^{A}, \mathbf{H}\right] \cos \left[\vec{q} \cdot \vec{\sigma}+\mathbf{B}_{\vec{q}}\left(\tau ; X_{\phi}^{A}, \mathbf{K}\right]\right],
\end{aligned}
$$




$$
\begin{aligned}
\pi(\tau, \vec{\sigma})= & -i \int d \tilde{q} \omega(q) \sqrt{F^{\tau}(q) \omega(q) P_{\phi}^{\tau}-F(q) \vec{q} \cdot \vec{P}_{\phi}+\mathcal{D}_{\vec{q}} \mathbf{H}(\tau, \vec{q})} \\
& {\left[e^{-i\left[\omega(q)\left(\tau-X_{\phi}^{\tau}\right)-\vec{q} \cdot\left(\vec{\sigma}-\vec{X}_{\phi}\right)\right]+i \int d \tilde{k} \int d \tilde{k}^{\prime} \mathbf{K}(\tau, \vec{k}) \mathcal{G}\left(\vec{k}, \vec{k}^{\prime}\right) \Delta\left(\vec{k}^{\prime}, \vec{q}\right)}-\right.} \\
& -e^{\left.+i\left[\omega(q)\left(\tau-X_{\phi}^{\tau}\right)-\vec{q} \cdot\left(\vec{\sigma}-\vec{X}_{\phi}\right)\right]-i \int d \tilde{k} \int d \tilde{k}^{\prime} \mathbf{K}(\tau, \vec{k}) \mathcal{G}\left(\vec{k}, \vec{k}^{\prime}\right) \Delta\left(\vec{k}^{\prime}, \vec{q}\right)\right]=} \\
= & -2 \int d \tilde{q} \omega(q) \mathbf{A}_{\vec{q}}\left(\tau ; P_{\phi}^{A}, \mathbf{H}\right] \sin \left[\vec{q} \cdot \vec{\sigma}+\mathbf{B}_{\vec{q}}\left(\tau ; X_{\phi}^{A}, \mathbf{K}\right]\right]
\end{aligned}
$$

$$
\begin{aligned}
\mathbf{A}_{\vec{q}}\left(\tau ; P_{\phi}^{A}, \mathbf{H}\right] & =\sqrt{F^{\tau}(q) \omega(q) P_{\phi}^{\tau}-F(q) \vec{q} \cdot \vec{P}_{\phi}+\mathcal{D}_{\vec{q}} \mathbf{H}(\tau, \vec{q})}=\sqrt{I(\tau, \vec{q})}, \\
\mathbf{B}_{\vec{q}}\left(\tau ; X_{\phi}^{A}, \mathbf{K}\right] & =-\vec{q} \cdot \vec{X}_{\phi}-\omega(q)\left(\tau-X_{\phi}^{\tau}\right)+\int d \tilde{k} d \tilde{k}^{\prime} \mathbf{K}(\tau, \vec{k}) \mathcal{G}\left(\vec{k}, \vec{k}^{\prime}\right) \triangle\left(\vec{k}^{\prime}, \vec{q}\right)= \\
& =\varphi(\tau, \vec{q})-\omega(q) \tau .
\end{aligned}
$$

The Klein-Gordon field configuration is described by:

i) its energy $P_{\phi}^{\tau}$ and the conjugate field time-variable $X_{\phi}^{\tau}$, which is equal to $\tau$ plus some kind of internal time $\tilde{X}_{\phi}^{\tau}$;

ii) the conjugate reduced canonical variables of a free point $\vec{X}_{\phi}, \vec{P}_{\phi}$;

iii) an infinite set of canonically conjugate relative variables $\mathbf{H}(\tau, \vec{q}), \mathbf{K}(\tau, \vec{q})$.

While the sets i) and ii) describe a "monopole" field configuration see Section V), which depends only on 8 degrees of freedom like a scalar particle at rest $\left[\vec{P}_{\phi} \approx 0\right]$ and with mass $\epsilon_{s} \approx \sqrt{\left(P_{\phi}^{\tau}\right)^{2}-\vec{P}_{\phi}^{2}} \approx P_{\phi}^{\tau}$, corresponding to the decoupled collective variables of the field configuration, the set iii) describes an infinite set of "canonical relative variables" with respect to the relativistic collective variables of the sets i) and ii).

The conditions $\mathbf{H}(\tau, \vec{q})=\mathbf{K}(\tau, \vec{q})=0$ select the class of field configurations, solutions of the Klein-Gordon equation, which are of the "monopole" type on the Wigner hyperplanes

$$
\begin{aligned}
\phi_{\text {mon }}(\tau, \vec{\sigma}) & =2 \int d \tilde{q} \sqrt{F^{\tau}(q) \omega(q) P_{\phi}^{\tau}-F(q) \vec{q} \cdot \vec{P}_{\phi}} \cos \left[\vec{q} \cdot\left(\vec{\sigma}-\vec{X}_{\phi}\right)-\omega(q)\left(\tau-X_{\phi}^{\tau}\right)\right] \approx \\
& \approx 2 \sqrt{P_{\phi}^{\tau}} \int d \tilde{q} \sqrt{F^{\tau}(q) \omega(q)} \cos \left[\vec{q} \cdot\left(\vec{\sigma}-\vec{X}_{\phi}\right)-\omega(q)\left(\tau-X_{\phi}^{\tau}\right)\right], \\
\pi_{\text {mon }}(\tau, \vec{\sigma}) & =-2 \int d \tilde{q} \sqrt{F^{\tau}(q) \omega(q) P_{\phi}^{\tau}-F(q) \vec{q} \cdot \vec{P}_{\phi}} \sin \left[\vec{q} \cdot\left(\vec{\sigma}-\vec{X}_{\phi}\right)-\omega(q)\left(\tau-X_{\phi}^{\tau}\right)\right] \approx \\
& \approx-2 \sqrt{P_{\phi}^{\tau}} \int d \tilde{q} \sqrt{F^{\tau}(q) \omega(q)} \sin \left[\vec{q} \cdot\left(\vec{\sigma}-\vec{X}_{\phi}\right)-\omega(q)\left(\tau-X_{\phi}^{\tau}\right)\right] .
\end{aligned}
$$

If we add the gauge-fixings $\vec{X}_{\phi} \approx 0$ to $\vec{P}_{\phi} \approx 0$ [this implies $\vec{\lambda}(\tau)=0$ in Eq. 2.10)] and go to Dirac brackets, the rest-frame instant-form Klein-Gordon canonical variables in the gauge $\tau \equiv T_{s}=p_{s} \cdot x_{s} / \epsilon_{s}$ (see the end of Section II) are [in the following formulas one has $\left.T_{s}-X_{\phi}^{\tau}=-\tilde{X}_{\phi}^{\tau}\right]$

$$
\begin{aligned}
a\left(T_{s}, \vec{q}\right)= & \sqrt{F^{\tau}(q) \omega(q) P_{\phi}^{\tau}+\mathcal{D}_{\vec{q}} \mathbf{H}\left(T_{s}, \vec{q}\right)} \\
& e^{i\left[\omega(q) \tilde{X}_{\phi}^{\tau}+\vec{q} \cdot \vec{\sigma}\right]+i \int d \tilde{k} \int d \tilde{k}^{\prime} \mathbf{K}\left(T_{s}, \vec{k}\right) \mathcal{G}\left(\vec{k}, \vec{k}^{\prime}\right) \Delta\left(\vec{k}^{\prime}, \vec{q}\right)}, \\
N_{\phi}= & \tilde{c} \frac{P_{\phi}^{\tau}}{m}+\int d \tilde{q} \mathcal{D}_{\vec{q}} \mathbf{H}\left(T_{s}, \vec{q}\right),
\end{aligned}
$$




$$
\begin{aligned}
& \phi\left(T_{s}, \vec{\sigma}\right)=\int d \tilde{q} \sqrt{F^{\tau}(q) \omega(q) P_{\phi}^{\tau}+\mathcal{D}_{\vec{q}} \mathbf{H}\left(T_{s}, \vec{q}\right)} \\
& {\left[e^{i\left[\omega(q) \tilde{X}_{\phi}^{\tau}+\vec{q} \cdot \vec{\sigma}\right]+i \int d \tilde{k} \int d \tilde{k}^{\prime} \mathbf{K}\left(T_{s}, \vec{k}\right) \mathcal{G}\left(\vec{k}, \vec{k}^{\prime}\right) \Delta\left(\vec{k}^{\prime}, \vec{q}\right)}+\right.} \\
& +e^{\left.-i\left[\omega(q) \tilde{X}_{\phi}^{\tau}+\vec{q} \cdot \vec{\sigma}\right]-i \int d \tilde{k} \int d \tilde{k}^{\prime} \mathbf{K}\left(T_{s}, \vec{k}\right) \mathcal{G}\left(\vec{k}, \vec{k}^{\prime}\right) \Delta\left(\vec{k}^{\prime}, \vec{q}\right)\right]}= \\
& =2 \int d \tilde{q} \mathbf{A}_{\vec{q}}\left(T_{s} ; P_{\phi}^{\tau}, \mathbf{H}\right] \cos \left[\vec{q} \cdot \vec{\sigma}+\mathbf{B}_{\vec{q}}\left(T_{s} ; \tilde{X}_{\phi}^{\tau}, \mathbf{K}\right]\right], \\
& \pi\left(T_{s}, \vec{\sigma}\right)=-i \int d \tilde{q} \omega(q) \sqrt{F^{\tau}(q) \omega(q) P_{\phi}^{\tau}+\mathcal{D}_{\vec{q}} \mathbf{H}\left(T_{s}, \vec{q}\right)} \\
& {\left[e^{i\left[\omega(q) \tilde{X}_{\phi}^{\tau}+\vec{q} \cdot \vec{\sigma}\right]+i \int d \tilde{k} \int d \tilde{k}^{\prime} \mathbf{K}\left(T_{s}, \vec{k}\right) \mathcal{G}\left(\vec{k}, \vec{k}^{\prime}\right) \Delta\left(\vec{k}^{\prime}, \vec{q}\right)}-\right.} \\
& -e^{\left.-i\left[\omega(q) \tilde{X}_{\phi}^{\tau}+\vec{q} \cdot \vec{\sigma}\right]-i \int d \tilde{k} \int d \tilde{k}^{\prime} \mathbf{K}\left(T_{s}, \vec{k}\right) \mathcal{G}\left(\vec{k}, \vec{k}^{\prime}\right) \Delta\left(\vec{k}^{\prime}, \vec{q}\right)\right]}= \\
& =-2 \int d \tilde{q} \omega(q) \mathbf{A}_{\vec{q}}\left(T_{s} ; P_{\phi}^{\tau}, \mathbf{H}\right] \sin \left[\vec{q} \cdot \vec{\sigma}+\mathbf{B}_{\vec{q}}\left(T_{s} ; \tilde{X}_{\phi}^{\tau}, \mathbf{K}\right]\right] \text {, }
\end{aligned}
$$

$$
\begin{aligned}
\mathbf{A}_{\vec{q}}\left(T_{s} ; P_{\phi}^{\tau}, \mathbf{H}\right]= & \sqrt{F^{\tau}(q) \omega(q) P_{\phi}^{\tau}+\mathcal{D}_{\vec{q}} \mathbf{H}\left(T_{s}, \vec{q}\right)}=\sqrt{I\left(T_{s}, \vec{q}\right)}, \\
\mathbf{B}_{\vec{q}}\left(T_{s} ; X_{\phi}^{\tau}, \mathbf{K}\right]= & \int d \tilde{k} d \tilde{k}^{\prime} \mathbf{K}\left(T_{s}, \vec{k}\right) \mathcal{G}\left(\vec{k}, \vec{k}^{\prime}\right) \triangle\left(\vec{k}^{\prime}, \vec{q}\right)+\omega(q) \tilde{X}_{\phi}^{\tau}= \\
= & \varphi\left(T_{s}, \vec{q}\right)-\omega(q) T_{s}, \\
& \Downarrow
\end{aligned}
$$$$
\frac{\partial \phi\left(T_{s}, \vec{\sigma}\right)}{\partial P_{\phi}^{\tau}}=\int d \tilde{q} F^{\tau}(q) \omega(q) \frac{\cos \left[\vec{q} \cdot \vec{\sigma}+\mathbf{B}_{\vec{q}}\left(T_{s} ; \tilde{X}_{\phi}^{\tau}, \mathbf{K}\right]\right]}{\mathbf{A}_{\vec{q}}\left(T_{s} ; P_{\phi}^{\tau}, \mathbf{K}\right]},
$$$$
\frac{\partial \phi\left(T_{s}, \vec{\sigma}\right)}{\partial X_{\phi}^{\tau}}=2 \int d \tilde{q} \omega(q) \mathbf{A}_{\vec{q}}\left(T_{s} ; P_{\phi}^{\tau}, \mathbf{H}\right] \sin \left[\vec{q} \cdot \vec{\sigma}+\mathbf{B}_{\vec{q}}\left(T_{s} ; \tilde{X}_{\phi}^{\tau}, \mathbf{K}\right]\right] \text {, }
$$$$
\frac{\delta \phi\left(T_{s}, \vec{\sigma}\right)}{\delta \mathbf{K}\left(T_{s}, \vec{q}\right)}=2 \int d \tilde{k} \mathbf{A}_{\vec{k}}\left(T_{s} ; P_{\phi}^{\tau}, \mathbf{H}\right]
$$$$
\sin \left[\vec{k} \cdot \vec{\sigma}+\mathbf{B}_{\vec{k}}\left(T_{s} ; \tilde{X}_{\phi}^{\tau}, \mathbf{K}\right]\right] \int d \tilde{k}^{\prime} \mathcal{G}\left(\vec{q}, \vec{k}^{\prime}\right) \triangle\left(\vec{k}^{\prime}, \vec{k}\right),
$$$$
\frac{\delta \phi\left(T_{s}, \vec{\sigma}\right)}{\delta \mathbf{H}\left(T_{s}, \vec{q}\right)}=\int d \tilde{k}\left(\frac{\cos \left[\vec{k} \cdot \vec{\sigma}+\mathbf{B}_{\vec{k}}\left(T_{s} ; \tilde{X}_{\phi}^{\tau}, \mathbf{K}\right]\right]}{\mathbf{A}_{\vec{k}}\left(T_{s} ; P_{\phi}^{\tau}, \mathbf{H}\right]}\right) \mathcal{D}_{\vec{k}} \delta^{3}(\vec{q}-\vec{k})=
$$$$
=-\mathcal{D}_{\vec{q}}\left(\frac{\cos \left[\vec{q} \cdot \vec{\sigma}+\mathbf{B}_{\vec{q}}\left(T_{s} ; \tilde{X}_{\phi}^{\tau}, \mathbf{K}\right]\right]}{\mathbf{A}_{\vec{q}}\left(T_{s} ; P_{\phi}^{\tau}, \mathbf{H}\right]}\right) .
$$

From Eq.(2.10), the Hamiltonian is now $M_{\phi}=P_{\phi}^{\tau}$ : it generates the following evolution in $T_{s}$

$$
\begin{aligned}
& \frac{\partial}{\partial T_{s}} X_{\phi}^{\tau} \stackrel{\circ}{=}\left\{X_{\phi}^{\tau}, P_{\phi}^{\tau}\right\}=-1, \quad \Rightarrow \quad X_{\phi}^{\tau} \stackrel{\circ}{=}-T_{s}, \\
& \frac{\partial}{\partial T_{s}} P_{\phi}^{\tau} \stackrel{\circ}{=} 0, \\
& \frac{\partial}{\partial T_{s}} \mathbf{H}\left(T_{s}, \vec{q}\right) \stackrel{\circ}{=} 0,
\end{aligned}
$$




$$
\begin{aligned}
& \frac{\partial}{\partial T_{s}} \mathbf{K}\left(T_{s}, \vec{q}\right) \stackrel{\circ}{=} 0
\end{aligned}
$$

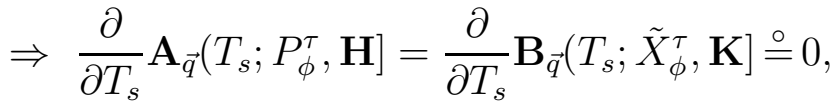

$$
\begin{aligned}
& \frac{\partial}{\partial T_{s}} \phi\left(T_{s}, \vec{\sigma}\right) \stackrel{\circ}{=}-\frac{\partial}{\partial X_{\phi}^{\tau}} \phi\left(T_{s}, \vec{\sigma}\right)=\pi\left(T_{s}, \vec{\sigma}\right), \\
& \frac{\partial}{\partial T_{s}} \pi\left(T_{s}, \vec{\sigma}\right) \stackrel{\circ}{=}-\frac{\partial}{\partial X_{\phi}^{\tau}} \pi\left(T_{s}, \vec{\sigma}\right)=-\left[\triangle+m^{2}\right] \phi\left(T_{s}, \vec{\sigma}\right), \\
& \Rightarrow\left(\frac{\partial^{2}}{\partial T_{s}^{2}}-\frac{\partial^{2}}{\partial \vec{\sigma}^{2}}+m^{2}\right) \phi\left(T_{s}, \vec{\sigma}\right) \stackrel{\circ}{=} 0 .
\end{aligned}
$$

Therefore, in the free case $\mathbf{H}\left(T_{s}, \vec{q}\right), \mathbf{K}\left(T_{s}, \vec{q}\right)$ are constants of the motion [complete integrability and Liouville theorem for the Klein-Gordon field]. Since the canonical variable $P_{\phi}^{\tau}$ is the Hamiltonian for the evolution in $T_{s} \equiv \tau$, we need the "internal" variable $X_{\phi}^{\tau}=\tau+\tilde{X}_{\phi}^{\tau}$ [i.e. the "internal time variable" $\left.\tilde{X}_{\phi}^{\tau}\right]$ to write Hamilton's equations $\frac{\partial}{\partial T_{s}} F \stackrel{\circ}{=}\left\{F, P_{\phi}^{\tau}\right\}=$ $-\frac{\partial F}{\partial X_{\phi}^{\tau}}=-\frac{\partial F}{\partial \tilde{X}_{\phi}^{\tau}}$; in the free case we have $\frac{\partial}{\partial T_{s}} \stackrel{\circ}{=}-\frac{\partial}{\partial X_{\phi}^{\tau}}$ on $\phi\left(T_{s}, \vec{\sigma}\right)\left[X_{\phi}^{\tau}, P_{\phi}^{\tau}, \mathbf{H}, \mathbf{K}\right]$ and $\pi\left(T_{s}, \vec{\sigma}\right)\left[X_{\phi}^{\tau}, P_{\phi}^{\tau}, \mathbf{H}, \mathbf{K}\right]$, so that the evolution in the time $X_{\phi}^{\tau}=T_{s}+\tilde{X}_{\phi}^{\tau}$ [which takes place inside the Wigner hyperplane and which can be interpreted as an evolution in the internal time $\tilde{X}_{\phi}^{\tau}$ ] is equal and opposite to the evolution in the rest-frame time $T_{s}$ from a Wigner hyperplane to the next one in the free case.

By adding the two second class constraints $X_{\phi}^{\tau}-T_{s}=\tilde{X}_{\phi}^{\tau} \approx 0, P_{\phi}^{\tau}-$ const. $\approx 0$, and by going to Dirac brackets, we get the rest-frame Hamilton-Jacobi formulation corresponding to the given constant value of the total energy [the field $\phi\left(T_{s}, \vec{\sigma}\right)$, which is $T_{s}$-independent since it depends only on the internal time $\tilde{X}_{\phi}^{\tau}$, now becomes also $\tilde{X}_{\phi}^{\tau}$ - independent]. In this way we find a symplectic subspace (spanned by the canonical variables $\mathbf{H}, \mathbf{K}$ ) of each constant energy $\left[P_{\phi}^{\tau}=\right.$ const.] surface of the Klein-Gordon field. Each constant energy surface is not a symplectic manifold, but in this way it turns out to be the disjoint union (over $\tilde{X}_{\phi}^{\tau}$ ) of the symplectic manifolds determined by $\tilde{X}_{\phi}^{\tau}=$ const., $P_{\phi}^{\tau}=$ const.

\section{E. A family of canonical multipoles.}

From Eqs.(4.39) we get [assuming that we can interchange the sums with the integrals]

$$
\begin{aligned}
\phi\left(T_{s}, \vec{\sigma}\right) & =2 \int d \tilde{q} \mathbf{A}_{\vec{q}}\left(T_{s} ; P_{\phi}^{\tau}, \mathbf{H}\right]\left(\cos \vec{q} \cdot \vec{\sigma} \cos \mathbf{B}_{\vec{q}}\left(T_{s} ; \tilde{X}_{\phi}^{\tau}, \mathbf{K}\right]-\right. \\
& \left.-\sin \vec{q} \cdot \vec{\sigma} \sin \mathbf{B}_{\vec{q}}\left(T_{s} ; \tilde{X}_{\phi}^{\tau}, \mathbf{K}\right]\right)= \\
& =\sum_{k=0}^{\infty}(-)^{k}\left[\frac{\sigma^{r_{1}} \ldots \sigma^{r_{2 k+1}}}{(2 k+1) !} \mathcal{T}_{\phi, O}^{r_{1} \ldots r_{2 k+1}}-\frac{\sigma^{r_{1}} \ldots \sigma^{r_{2 k}}}{(2 k) !} \mathcal{T}_{\phi, E}^{r_{1} . r_{2 k}}\right], \\
\pi\left(T_{s}, \vec{\sigma}\right) & =-2 \int d \tilde{q} \omega(q) \mathbf{A}_{\vec{q}}\left(T_{s} ; P_{\phi}^{\tau}, \mathbf{H}\right]\left(\sin \vec{q} \cdot \vec{\sigma} \cos \mathbf{B}_{\vec{q}}\left(T_{s} ; \tilde{X}_{\phi}^{\tau}, \mathbf{K}\right]+\right. \\
& \left.+\cos \vec{q} \cdot \vec{\sigma} \sin \mathbf{B}_{\vec{q}}\left(T_{s} ; \tilde{X}_{\phi}^{\tau}, \mathbf{K}\right]\right)=
\end{aligned}
$$




$$
\begin{aligned}
& =-\sum_{k=0}^{\infty}(-)^{k}\left[\frac{\sigma^{r_{1}} \ldots \sigma^{r_{2 k+1}}}{(2 k+1) !} \mathcal{T}_{\pi, O}^{r_{1} \ldots r_{2 k+1}}-\frac{\sigma^{r_{1}} \ldots \sigma^{r_{2 k}}}{(2 k) !} \mathcal{T}_{\pi, E}^{r_{1} . r_{2 k}}\right], \\
\mathcal{T}_{\phi, O}^{r_{1} \ldots r_{2 k+1}} & =2 \int d \tilde{q} q^{r_{1}} \ldots q^{r_{2 k+1}} \mathbf{A}_{\vec{q}}\left(T_{s} ; P_{\phi}^{\tau}, \mathbf{H}\right] \cos \mathbf{B}_{\vec{q}}\left(T_{s} ; \tilde{X}_{\phi}^{\tau}, \mathbf{K}\right], \\
\mathcal{T}_{\phi, E}^{r_{1} . . r_{2 k}} & =2 \int d \tilde{q} q^{r_{1}} \ldots q^{r_{2 k}} \mathbf{A}_{\vec{q}}\left(T_{s} ; P_{\phi}^{\tau}, \mathbf{H}\right] \sin \mathbf{B}_{\vec{q}}\left(T_{s} ; \tilde{X}_{\phi}^{\tau}, \mathbf{K}\right], \\
\mathcal{T}_{\pi, O}^{r_{1} \ldots r_{2 k+1}} & =2 \int d \tilde{q} \omega(q) q^{r_{1}} \ldots q^{r_{2 k+1}} \mathbf{A}_{\vec{q}}\left(T_{s} ; P_{\phi}^{\tau}, \mathbf{H}\right] \sin \mathbf{B}_{\vec{q}}\left(T_{s} ; \tilde{X}_{\phi}^{\tau}, \mathbf{K}\right], \\
\mathcal{T}_{\pi, E}^{r_{1} . . r_{2 k}} & =2 \int d \tilde{q} \omega(q) q^{r_{1} \ldots} \ldots q^{r_{2 k}} \mathbf{A}_{\vec{q}}\left(T_{s} ; P_{\phi}^{\tau}, \mathbf{H}\right] \cos \mathbf{B}_{\vec{q}}\left(T_{s} ; \tilde{X}_{\phi}^{\tau}, \mathbf{K}\right] .
\end{aligned}
$$

In this way we have defined a set of canonical multipoles of the fields $\phi\left(T_{s}, \vec{\sigma}\right), \pi\left(T_{s}, \vec{\sigma}\right)$, with respect to $\vec{\sigma}=0$, i.e. with respect to the origin $x_{s}^{\mu}(\tau)$ of the Wigner hyperplanes (a noncanonical covariant centroid) and they are expressed in terms of the final canonical basis for the Klein-Gordon field.

When the fields have a compact support $\mathrm{W}$ in momentum space, it can be shown that the only nonvanishing Poisson brackets of these multipoles are

$$
\begin{aligned}
\left\{\mathcal{T}_{\pi, O}^{r_{1} \ldots r_{2 k+1}}, \mathcal{T}_{\phi, O}^{s_{1} \ldots s_{2 h+1}}\right\} & =\mathcal{I}^{r_{1} \ldots r_{2 k+1}, s_{1} \ldots s_{2 h+1}}, \\
\left\{\mathcal{T}_{\pi, E}^{r_{1} \ldots r_{2 k}, O} \mathcal{T}_{\phi, E}^{s_{1} \ldots s_{2 h}}\right\} & =-\mathcal{I}^{r_{1} \ldots r_{2 k}, s_{1} \ldots s_{2 h}}, \\
\mathcal{I}^{r_{1} \ldots r_{m}, s_{1} \ldots s_{n}} & =2 \int_{W} d \tilde{q} \omega^{2}(q) q^{r_{1}} \cdots q^{r_{m}} q^{s_{1}} \cdots q^{s_{n}} .
\end{aligned}
$$

That is they form a closed algebra with a generalized Kronecker symbol, which could be quantized instead of the Fourier coefficients.

\section{F. Effects of self interactions $V(\phi)$.}

When there is an interaction term $V(\phi)$, the Hamiltonian becomes $M_{\phi}=\tilde{P}_{\phi}^{\tau}=P_{\phi}^{\tau}+$ $\int d^{3} \sigma V(\phi)\left(T_{s}, \vec{\sigma}\right)$ and one has [see the difference between the rest-frame time evolution and the internal time one in presence of interactions]

$$
\begin{aligned}
\frac{\partial}{\partial T_{s}} X_{\phi}^{\tau} & \stackrel{\circ}{=}-1-\frac{\partial}{\partial P_{\phi}^{\tau}} \int d^{3} \sigma V\left(\phi\left(T_{s}, \vec{\sigma}\right)\right)= \\
& =-1-\int d \tilde{k} \frac{F^{\tau}(k) \omega(k)}{\mathbf{A}_{\vec{k}}\left(T_{s} ; P_{\phi}^{\tau}, \mathbf{H}\right]} \int d^{3} \sigma V^{\prime}\left(\phi\left(T_{s}, \vec{\sigma}\right)\right) \cos \left[\vec{k} \cdot \vec{\sigma}+\mathbf{B}_{\vec{k}}\left(T_{s} ; X_{\phi}^{\tau}, \mathbf{K}\right]\right], \\
\frac{\partial}{\partial T_{s}} \tilde{P}_{\phi}^{\tau} & \stackrel{\circ}{=} 0, \\
\frac{\partial}{\partial T_{s}} P_{\phi}^{\tau} & \stackrel{\circ}{=} \frac{\partial}{\partial X_{\phi}^{\tau}} \int d^{3} \sigma V\left(\phi\left(T_{s}, \vec{\sigma}\right)\right)= \\
& =-2 \int d \tilde{k} \omega(k) \mathbf{A}_{\vec{k}}\left(T_{s} ; P_{\phi}^{\tau}, \mathbf{H}\right] \int d^{3} \sigma V^{\prime}\left(\phi\left(T_{s}, \vec{\sigma}\right)\right) \sin \left[\vec{k} \cdot \vec{\sigma}+\mathbf{B}\left(T_{s} ; X_{\phi}^{\tau}, \mathbf{K}\right]\right], \\
\frac{\partial}{\partial T_{s}} \mathbf{H}\left(T_{s}, \vec{q}\right) & \stackrel{\circ}{=} \frac{\delta}{\delta \mathbf{K}\left(T_{s}, \vec{q}\right)} \int d^{3} \sigma V\left(\phi\left(T_{s}, \vec{\sigma}\right)\right)=
\end{aligned}
$$




$$
\begin{aligned}
= & -2 \int d^{3} \sigma V^{\prime}\left(\phi\left(T_{s}, \vec{\sigma}\right)\right) \int d \tilde{k} \mathbf{A}_{\vec{k}}\left(T_{s} ; P_{\phi}^{\tau}, \mathbf{H}\right] \\
& \sin \left[\vec{k} \cdot \vec{\sigma}+\mathbf{B}_{\vec{k}}\left(T_{s} ; X_{\phi}^{\tau}, \mathbf{K}\right]\right] \int d \tilde{k}^{\prime} \mathcal{G}\left(\vec{q}, \vec{k}^{\prime}\right) \triangle\left(\vec{k}^{\prime}, \vec{k}\right), \\
\frac{\partial}{\partial T_{s}} \mathbf{K}\left(T_{s}, \vec{q}\right) \stackrel{\circ}{=} & -\frac{\delta}{\delta \mathbf{H}\left(T_{s}, \vec{q}\right)} \int d^{3} \sigma V\left(\phi\left(T_{s}, \vec{\sigma}\right)\right)= \\
= & -\int d^{3} \sigma V^{\prime}\left(\phi\left(T_{s}, \vec{\sigma}\right)\right) \mathcal{D}_{\vec{q}}\left(\frac{\cos \left[\vec{q} \cdot \vec{\sigma}+\mathbf{B}_{\vec{q}}\left(T_{s} ; X_{\phi}^{\tau}, \mathbf{K}\right]\right]}{\mathbf{A}_{\vec{q}}\left(T_{s} ; P_{\phi}^{\tau}, \mathbf{H}\right]}\right),
\end{aligned}
$$

with $V^{\prime}(\phi)=\partial V(\phi) / \partial \phi$. In particular we get

$$
\begin{aligned}
& \frac{\partial}{\partial T_{s}} \mathbf{A}_{\vec{q}}\left(T_{s} ; P_{\phi}^{\tau}, \mathbf{H}\right]=\frac{1}{2 \mathbf{A}_{\vec{q}}\left(T_{s} ; P_{\phi}^{\tau}, \mathbf{H}\right]}\left[F^{\tau}(q) \omega(q) \frac{\partial P_{\phi}^{\tau}}{\partial T_{s}}+\mathcal{D}_{\vec{q}} \frac{\partial \mathbf{H}\left(T_{s}, \vec{q}\right)}{\partial T_{s}}\right] \stackrel{\circ}{=} \\
& \stackrel{\circ}{=}-\frac{1}{\mathbf{A}_{\vec{q}}\left(T_{s} ; P_{\phi}^{\tau}, \mathbf{H}\right]} \int d^{3} \sigma V^{\prime}\left(\phi\left(T_{s}, \vec{\sigma}\right)\right) \int d \tilde{k}\left[F^{\tau}(q) \omega(q) \omega(k)+\triangle(\vec{q}, \vec{k})\right] \\
& \mathbf{A}_{\vec{k}}\left(T_{s} ; P_{\phi}^{\tau}, \mathbf{H}\right] \sin \left[\vec{k} \cdot \vec{\sigma}+\mathbf{B}_{\vec{k}}\left(T_{s} ; X_{\phi}^{\tau}, \mathbf{K}\right]\right]= \\
& =-\frac{1}{\mathbf{A}_{\vec{q}}\left(T_{s} ; P_{\phi}^{\tau}, \mathbf{H}\right]} \int d \tilde{k} \mathbf{A}_{\vec{k}}\left(T_{s} ; P_{\phi}^{\tau}, \mathbf{H}\right] \int d^{3} \sigma V^{\prime}\left(\phi\left(T_{s}, \vec{\sigma}\right)\right) \\
& \sin \left[\vec{k} \cdot \vec{\sigma}+\mathbf{B}_{\vec{k}}\left(T_{s} ; X_{\phi}^{\tau}, \mathbf{K}\right]\right]\left[\Omega(k) \delta^{3}(\vec{q}-\vec{k})+F(q) \vec{q} \cdot \vec{k}\right], \\
& \frac{\partial}{\partial T_{s}} \mathbf{B}_{\vec{q}}\left(T_{s} ; X_{\phi}^{\tau}, \mathbf{K}\right]=-\omega(q)\left(1-\frac{\partial X_{\phi}^{\tau}}{\partial T_{s}}\right)+\int d \tilde{k} d \tilde{k}^{\prime} \frac{\partial \mathbf{K}\left(T_{s}, \vec{k}\right)}{\partial T_{s}} \mathcal{G}\left(\vec{k}, \vec{k}^{\prime}\right) \triangle\left(\vec{k}^{\prime}, \vec{q}\right) \stackrel{\circ}{=} \\
& \stackrel{\circ}{=}-2 \omega(q)-\int d^{3} \sigma V^{\prime}\left(\phi\left(T_{s}, \vec{\sigma}\right)\right) \\
& \int d \tilde{k}\left[\omega(q) F^{\tau}(k) \omega(k)+\triangle(\vec{k}, \vec{q})\right] \frac{\cos \left[\vec{k} \cdot \vec{\sigma}+\mathbf{B}_{\vec{k}}\left(T_{s} ; X_{\phi}^{\tau}, \mathbf{K}\right]\right]}{\mathbf{A}_{\vec{k}}\left(T_{s} ; P_{\phi}^{\tau}, \mathbf{H}\right]}= \\
& =-2 \omega(q)-\int d \tilde{k} \frac{\Omega(k) \delta^{3}(\vec{q}-\vec{k})+F(k) \vec{q} \cdot \vec{k}}{\mathbf{A}\left(T_{s} ; P_{\phi}^{\tau}, \mathbf{H}\right]} \\
& \int d^{3} \sigma V^{\prime}\left(\phi\left(T_{s}, \vec{\sigma}\right)\right) \cos \left[\vec{k} \cdot \vec{\sigma}+\mathbf{B}_{\vec{k}}\left(T_{s} ; X_{\phi}^{\tau}, \mathbf{K}\right]\right] .
\end{aligned}
$$

For $V(\phi)=\frac{1}{n+1} \xi \phi^{n+1}$ we have:

$V^{\prime}\left(\phi\left(T_{s}, \vec{\sigma}\right)\right)=\xi \phi^{n}\left(T_{s}, \vec{\sigma}\right)=2^{n} \xi \int d \tilde{q}_{1} . . d \tilde{q}_{n} \mathbf{A}_{\vec{q}_{1}}\left(T_{s} ; P_{\phi}^{\tau}, \mathbf{H}\right] \ldots \mathbf{A}_{\vec{q}_{n}}\left(T_{s} ; P_{\phi}^{\tau}, \mathbf{H}\right] \cos \left(\vec{q}_{1} \cdot \vec{\sigma}+\right.$ $\left.\mathbf{B}_{\vec{q}_{1}}\left(T_{s} ; \tilde{X}_{\phi}^{\tau}, \mathbf{K}\right]\right) \ldots \cos \left(\vec{q}_{n} \cdot \vec{\sigma}+\mathbf{B}_{\vec{q}_{n}}\left(T_{s} ; \tilde{X}_{\phi}^{\tau}, \mathbf{K}\right]\right)$.

For the sine-Gordon case we have $V(\phi)=\frac{\mu^{4}}{\lambda}\left[\cos \left(\frac{\sqrt{\lambda}}{\mu}-1\right]\right.$ and $V^{\prime}\left(\phi\left(T_{s}, \vec{\sigma}\right)\right)=$ $\frac{\mu^{3}}{\sqrt{\lambda}} \sin \left(\frac{\sqrt{\lambda}}{\mu} \phi\left(T_{s}, \vec{\sigma}\right)\right)=\frac{\mu^{3}}{\sqrt{\lambda}} \sin \left(2 \frac{\sqrt{\lambda}}{\mu} \int d \tilde{q} \mathbf{A}_{\vec{q}}\left(T_{s} ; P_{\phi}^{\tau}, \mathbf{H}\right] \cos \left[\vec{q} \cdot \vec{\sigma}+\mathbf{B}_{\vec{q}}\left(T_{s} ; \tilde{X}_{\phi}^{\tau}, \mathbf{K}\right]\right]\right)$.

With a completely integrable interaction, it should exist a new canonical basis $\hat{\tilde{X}}_{\phi}^{\tau}, \hat{P}_{\phi}^{\tau}$, $\hat{\mathbf{H}}\left(T_{s}, \vec{q}\right), \hat{\mathbf{K}}\left(T_{s}, \vec{q}\right)$ with $\hat{\tilde{X}}_{\phi}^{\tau}$ the real "internal" time like in the free case.

For instance, in the new canonical basis of Eqs. (4.35) one can define a family of completely integrable interactions associated with the Hamiltonian $M_{\phi}=P_{\phi}^{\tau}+$ 
$\int d \tilde{q}\left[\right.$ terms quadratic in $\mathbf{H}\left(T_{s}, \vec{q}\right)$ and $\left.\mathbf{K}\left(T_{s}, \vec{q}\right)\right]$. However, their associated Lagrangian density as functionals of $\phi(\tau, \vec{\sigma})$ would be completely non local first because the canonical transformation $\phi, \pi \mapsto X_{\phi}^{A}, P_{\phi}^{A}, \mathbf{H}, \mathbf{K}$ is non local, and second because one would have to solve an integral equation to get the momenta $\pi(\tau, \vec{\sigma})$ in terms of the velocities $\dot{\phi}(\tau, \vec{\sigma})$ $\left[\frac{\partial}{\partial T_{s}} \phi\left(T_{s}, \vec{\sigma}\right) \stackrel{\circ}{=}\left\{\phi\left(T_{s}, \vec{\sigma}\right), M_{\phi}\right\}\right]$. 


\section{THE ENERGY-MOMENTUM TENSOR ON WIGNER HYPERPLANES, DIXON'S MULTIPOLES AND THE CENTER OF MASS OF A FIELD CONFIGURATION.}

Let us now look at other properties of the Klein-Gordon field on the Wigner hyperplanes. In particular we are interested in identifying which kind of collective variables describe the center of mass of a field configuration and which is their relation, if any, with the previous collective variables. In so doing, we shall consider a field configuration as a relativistic extended body and we shall study its Dixon multipoles [9].

The Euler-Lagrange equations from the action (2.1) are

$$
\begin{aligned}
& \left(\frac{\partial \mathcal{L}}{\partial z^{\mu}}-\partial_{A} \frac{\partial \mathcal{L}}{\partial z_{A}^{\mu}}\right)(\tau, \vec{\sigma})=\eta_{\mu \nu} \partial_{A}\left[\sqrt{g} T^{A B}[\phi] z_{B}^{\nu}\right](\tau, \vec{\sigma}) \stackrel{\circ}{=} 0 \\
& \left(\frac{\partial \mathcal{L}}{\partial \phi}-\partial_{A} \frac{\partial \mathcal{L}}{\partial \partial_{A} \phi}\right)(\tau, \vec{\sigma}) \stackrel{\circ}{=} 0
\end{aligned}
$$

where we introduced the energy-momentum tensor

$$
\begin{aligned}
T^{A B}(\tau, \vec{\sigma})[\phi] & =-\left[\frac{2}{\sqrt{g}} \frac{\delta S}{\delta g_{A B}}\right](\tau, \vec{\sigma})= \\
& =\left[\partial^{A} \phi \partial^{B} \phi-\frac{1}{2} g^{A B}\left(\pi^{2}-(\vec{\partial} \phi)^{2}-m^{2} \phi^{2}\right)\right](\tau, \vec{\sigma}) .
\end{aligned}
$$

When $\partial_{A}\left[\sqrt{g} z_{B}^{\mu}\right]=0$, as it happens on the Wigner hyperplanes in the gauge $T_{s}-\tau \approx 0$, $\vec{\lambda}(\tau)=0$, we get the conservation of the energy-momentum tensor $T^{A B}$, i.e. $\partial_{A} T^{A B} \stackrel{\circ}{=}$. Otherwise, there is compensation coming from the dynamics of the surface.

The conserved, manifestly Lorentz covariant, energy-momentum tensor of the KleinGordon field $T^{\mu \nu}(x)[\tilde{\phi}]=-\frac{1}{2} \eta^{\mu \nu}\left[\partial_{\alpha} \tilde{\phi}(x) \partial^{\alpha} \tilde{\phi}(x)-m^{2} \tilde{\phi}^{2}(x)\right]+\partial^{\mu} \tilde{\phi}(x) \partial^{\nu} \tilde{\phi}(x)$ becomes $T^{A B}(\tau, \vec{\sigma})[\phi]$ in coordinates adapted to the hypersurface $\Sigma_{\tau}\left[\sigma^{A}=(\tau, \vec{\sigma}), q^{A}=(\omega(q), \vec{q})\right]$, where

$$
\begin{aligned}
T^{A B}(\tau, \vec{\sigma})[\phi] & =z_{\mu}^{A}(\tau, \vec{\sigma}) z_{\nu}^{B}(\tau, \vec{\sigma}) T^{\mu \nu}(z(\tau, \vec{\sigma}))[\tilde{\phi}]= \\
& =z_{\mu}^{A}(\tau, \vec{\sigma}) z_{\nu}^{B}(\tau, \vec{\sigma}) T^{\mu \nu}(\tau, \vec{\sigma})[\phi=\tilde{\phi} \circ z]= \\
& =-\frac{1}{2} g^{A B}(\tau, \vec{\sigma})\left[\pi^{2}-(\vec{\partial} \phi)^{2}-m^{2} \phi^{2}\right](\tau, \vec{\sigma})+\partial^{A} \phi(\tau, \vec{\sigma}) \partial^{B} \phi(\tau, \vec{\sigma}) .
\end{aligned}
$$

By using the results in Ref. [1], we have the following expression for the energymomentum tensor

1) On arbitrary spacelike hypersurfaces we get

$$
\begin{aligned}
T^{\mu \nu}(\tau, \vec{\sigma})[\phi] & =-\frac{1}{2} \eta^{\mu \nu}\left[\partial_{A} \phi(\tau, \vec{\sigma}) \partial^{A} \phi(\tau, \vec{\sigma})-m^{2} \phi^{2}(\tau, \vec{\sigma})\right]+ \\
& +z_{A}^{\mu}(\tau, \vec{\sigma}) z_{B}^{\nu}(\tau, \vec{\sigma}) \partial^{A} \phi(\tau, \vec{\sigma}) \partial^{B} \phi(\tau, \vec{\sigma})= \\
& =\left[-\frac{1}{2} \eta^{\mu \nu} g^{\tau \tau}+\left(z_{\tau}^{\mu} g^{\tau \tau}+z_{u}^{\mu} g^{u \tau}\right)\left(z_{\tau}^{\nu} g^{\tau \tau}+z_{v}^{\nu} g^{v \tau}\right)\right](\tau, \vec{\sigma}) \pi^{2}(\tau, \vec{\sigma})+ \\
& +\left[-\eta^{\mu \nu} g^{\tau r}+\left(z_{\tau}^{\mu} g^{\tau \tau}+z_{u}^{\mu} g^{u \tau}\right)\left(z_{\tau}^{\nu} g^{\tau r}+z_{v}^{\nu} g^{v r}\right)+\right.
\end{aligned}
$$




$$
\begin{aligned}
& \left.+\left(z_{\tau}^{\mu} g^{\tau r}+z_{u}^{\mu} g^{u r}\right)\left(z_{\tau}^{\nu} g^{\tau \tau}+z_{v}^{\nu} g^{v \tau}\right)\right](\tau, \vec{\sigma})\left[\pi \partial_{r} \phi\right](\tau, \vec{\sigma})+ \\
& +\left[-\frac{1}{2} \eta^{\mu \nu} g^{r s}+\left(z_{\tau}^{\mu} g^{\tau r}+z_{u}^{\mu} g^{u r}\right)\left(z_{\tau}^{\nu} g^{\tau s}+z_{v}^{\nu} g^{v s}\right)\right](\tau, \vec{\sigma})\left[\partial_{r} \phi \partial_{s} \phi\right](\tau, \vec{\sigma})+ \\
& +\frac{1}{2} \eta^{\mu \nu} m^{2} \phi^{2}(\tau, \vec{\sigma}) .
\end{aligned}
$$

2) On arbitrary spacelike hyperplanes, where

$$
\begin{aligned}
z^{\mu}(\tau, \vec{\sigma}) & =x_{s}^{\mu}(\tau)+b_{u}^{\mu}(\tau) \sigma^{u}, \\
z_{r}^{\mu}(\tau, \vec{\sigma}) & =b_{r}^{\mu}(\tau), \quad z_{\tau}^{\mu}(\tau, \vec{\sigma})=\dot{x}_{s}^{\mu}(\tau)+\dot{b}_{u}^{\mu}(\tau) \sigma^{u}=l^{\mu} / \sqrt{g^{\tau \tau}}-g_{\tau r} z_{r}^{\mu}, \\
g_{\tau \tau} & \left.=\dot{x}_{s} \cdot \dot{b}_{r} \sigma^{r}\right]^{2}, \quad g_{\tau r}=b_{r \mu}\left[\dot{x}_{s}^{\mu}+\dot{b}_{s}^{\mu} \sigma^{s}\right], \\
g_{r s} & =-\delta_{r s}, \quad \gamma^{r s}=-\delta^{r s}, \quad \gamma=1, \\
g & =g_{\tau \tau}+\sum_{r} g_{\tau r}^{2}, \\
g^{\tau \tau} & =1 /\left[l_{\mu}\left(\dot{x}_{s}^{\mu}+\dot{b}_{u}^{\mu} \sigma^{u}\right)\right]^{2}, \quad g^{\tau r}=g^{\tau \tau} g_{\tau r}=b_{r \mu}\left(\dot{x}_{s}^{\mu}+\dot{b}_{u}^{\mu} \sigma^{u}\right) /\left[l_{\mu}\left(\dot{x}_{s}^{\mu}+\dot{b}_{u}^{\mu} \sigma^{u}\right)\right]^{2}, \\
g^{r s} & =-\delta^{r s}+g^{\tau \tau} g_{\tau r} g_{\tau s}=-\delta^{r s}+b_{r \mu}\left(\dot{x}_{s}^{\mu}+\dot{b}_{u}^{\mu} \sigma^{u}\right) b_{r \nu}\left(\dot{x}_{s}^{\nu}+\dot{b}_{v}^{\nu} \sigma^{v}\right) /\left[l_{\mu}\left(\dot{x}_{s}^{\mu}+\dot{b}_{u}^{\mu} \sigma^{u}\right)\right]^{2},
\end{aligned}
$$

there is no relevant simplification for $T^{\mu \nu}\left(x_{s}^{\beta}(\tau)+b_{u}^{\beta}(\tau) \sigma^{u}\right)[\phi]$.

3) On Wigner hyperplanes, where

$$
\begin{aligned}
z^{\mu}(\tau, \vec{\sigma}) & =x_{s}^{\mu}(\tau)+\epsilon_{u}^{\mu}\left(u\left(p_{s}\right)\right) \sigma^{u}, \\
z_{r}^{\mu} & =\epsilon_{r}^{\mu}\left(u\left(p_{s}\right), \quad l^{\mu}=u^{\mu}\left(p_{s}\right), \quad z_{\tau}^{\mu}=\dot{x}_{s}^{\mu}(\tau),\right. \\
g & =\left[\dot{x}_{s}(\tau) \cdot u\left(p_{s}\right)\right], \quad g_{\tau \tau}=\dot{x}_{s}^{2}, \quad g_{\tau r}=\dot{x}_{s \mu} \epsilon_{r}^{\mu}\left(u\left(p_{s}\right)\right), \quad g_{r s}=-\delta_{r s}, \\
g^{\tau \tau} & =1 /\left[\dot{x}_{s \mu} u^{\mu}\left(p_{s}\right)\right]^{2}, \quad g^{\tau r}=\dot{x}_{s \mu} \epsilon_{r}^{\mu}\left(u\left(p_{s}\right)\right) /\left[\dot{x}_{s \mu} u^{\mu}\left(p_{s}\right)\right]^{2}, \\
g^{r s} & =-\delta^{r s}+\dot{x}_{s \mu} \epsilon_{r}^{\mu}\left(u\left(p_{s}\right)\right) \dot{x}_{s \nu} \epsilon_{s}^{\nu}\left(u\left(p_{s}\right)\right) /\left[\dot{x}_{s \mu} u^{\mu}\left(p_{s}\right)\right]^{2},
\end{aligned}
$$

we get

$$
\begin{aligned}
T^{\mu \nu}\left[x_{s}^{\mu}(\tau)+\right. & \left.\epsilon_{u}^{\mu}\left(u\left(p_{s}\right)\right) \sigma^{u}\right][\phi]=\frac{1}{\left[\dot{x}_{s}(\tau) \cdot u\left(p_{s}\right)\right]^{2}}\left[-\frac{1}{2} \eta^{\mu \nu}+\right. \\
+ & {\left.\left[\dot{x}_{s}^{\mu}(\tau)+\dot{x}_{s \rho}(\tau) \epsilon_{u}^{\rho}\left(u\left(p_{s}\right)\right) \epsilon_{u}^{\mu}\left(u\left(p_{s}\right)\right)\right]\left[\dot{x}_{s}^{\nu}(\tau)+\dot{x}_{s \sigma}(\tau) \epsilon_{u}^{\sigma}\left(u\left(p_{s}\right)\right) \epsilon_{u}^{\nu}\left(u\left(p_{s}\right)\right)\right]\right] \pi^{2}(\tau, \vec{\sigma})+} \\
+ & \frac{1}{\left[\dot{x}_{s}(\tau) \cdot u\left(p_{s}\right)\right]^{2}}\left[\frac{1}{2} \eta^{\mu \nu}\left(\left[\dot{x}_{s}(\tau) \cdot u\left(p_{s}\right)\right]^{2} \delta_{r s}-\dot{x}_{s \alpha}(\tau) \epsilon_{r}^{\alpha}\left(u\left(p_{s}\right)\right) \dot{x}_{s \beta}(\tau) \epsilon_{s}^{\beta}\left(u\left(p_{s}\right)\right)\right)+\right. \\
+ & {\left[\dot{x}_{s}(\tau) \cdot u\left(p_{s}\right)\right]^{2}\left(\epsilon_{r}^{\mu}\left(u\left(p_{s}\right)\right)-\frac{\dot{x}_{s \beta}(\tau) \epsilon_{r}^{\beta}\left(u\left(p_{s}\right)\right)}{\left[\dot{x}_{s}(\tau) \cdot u\left(p_{s}\right)\right]^{2}}\left[\dot{x}_{s}^{\mu}(\tau)+\dot{x}_{s \alpha}(\tau) \epsilon_{u}^{\alpha}\left(u\left(p_{s}\right)\right) \epsilon_{u}^{\mu}\left(u\left(p_{s}\right)\right)\right]\right) } \\
& \left.\left(\epsilon_{r}^{\nu}\left(u\left(p_{s}\right)\right)-\frac{\dot{x}_{s \beta}(\tau) \epsilon_{r}^{\beta}\left(u\left(p_{s}\right)\right)}{\left[\dot{x}_{s}(\tau) \cdot u\left(p_{s}\right)\right]^{2}}\left[\dot{x}_{s}^{\nu}(\tau)+\dot{x}_{s \alpha}(\tau) \epsilon_{v}^{\alpha}\left(u\left(p_{s}\right)\right) \epsilon_{v}^{\nu}\left(u\left(p_{s}\right)\right)\right]\right)\right] \\
& {\left[\partial_{r} \phi \partial_{s} \phi\right](\tau, \vec{\sigma})+\frac{1}{2} \eta^{\mu \nu} m^{2} \phi^{2}(\tau, \vec{\sigma})+} \\
+ & \frac{1}{\left[\dot{x}_{s}(\tau) \cdot u\left(p_{s}\right)\right]^{2}}\left[-\eta^{\mu \nu} \dot{x}_{s \rho}(\tau) \epsilon_{r}^{\rho}\left(u\left(p_{s}\right)\right)-\right.
\end{aligned}
$$




$$
\begin{aligned}
- & {\left[\dot{x}_{s}^{\mu}(\tau)+\dot{x}_{s \rho}(\tau) \epsilon_{u}^{\rho}\left(u\left(p_{s}\right)\right) \epsilon_{u}^{\mu}\left(u\left(p_{s}\right)\right)\right] } \\
& \left(\epsilon_{r}^{\nu}\left(u\left(p_{s}\right)\right)-\frac{\dot{x}_{s \beta}(\tau) \epsilon_{r}^{\beta}\left(u\left(p_{s}\right)\right)}{\left[\dot{x}_{s}(\tau) \cdot u\left(p_{s}\right)\right]^{2}}\left[\dot{x}_{s}^{\nu}(\tau)+\dot{x}_{s \alpha}(\tau) \epsilon_{v}^{\alpha}\left(u\left(p_{s}\right)\right) \epsilon_{v}^{\nu}\left(u\left(p_{s}\right)\right)\right]\right)- \\
- & \left(\epsilon_{r}^{\mu}\left(u\left(p_{s}\right)\right)-\frac{\dot{x}_{s \beta}(\tau) \epsilon_{r}^{\beta}\left(u\left(p_{s}\right)\right)}{\left[\dot{x}_{s}(\tau) \cdot u\left(p_{s}\right)\right]^{2}}\left[\dot{x}_{s}^{\mu}(\tau)+\dot{x}_{s \alpha}(\tau) \epsilon_{u}^{\alpha}\left(u\left(p_{s}\right)\right) \epsilon_{u}^{\mu}\left(u\left(p_{s}\right)\right)\right]\right) \\
& {\left[\dot{x}_{s}^{\nu}(\tau)+\dot{x}_{s \rho}(\tau) \epsilon_{v}^{\rho}\left(u\left(p_{s}\right)\right) \epsilon_{v}^{\nu}\left(u\left(p_{s}\right)\right)\right]\left[\pi \partial_{r} \phi\right](\tau, \vec{\sigma}) . }
\end{aligned}
$$

Since we have

$$
\begin{aligned}
\dot{x}_{s}^{\mu}(\tau) & =-\lambda^{\mu}(\tau)=\left[u^{\mu}\left(p_{s}\right) u^{\nu}\left(p_{s}\right)-\epsilon_{r}^{\mu}\left(u\left(p_{s}\right)\right) \epsilon_{r}^{\nu}\left(u\left(p_{s}\right)\right)\right] \dot{x}_{s \nu}(\tau)= \\
& =-u^{\mu}\left(p_{s}\right) \lambda(\tau)+\epsilon_{r}^{\mu}\left(u\left(p_{s}\right)\right) \lambda_{r}(\tau), \\
\dot{x}_{s}^{2}(\tau) & =\lambda^{2}(\tau)-\vec{\lambda}(\tau)>0, \\
U_{s}^{\mu}(\tau) & =\frac{\dot{x}_{s}^{\mu}(\tau)}{\sqrt{\dot{x}_{s}^{2}(\tau)}}=\frac{-\lambda(\tau) u^{\mu}\left(p_{s}\right)+\lambda_{r}(\tau) \epsilon_{r}^{\mu}\left(u\left(p_{s}\right)\right)}{\sqrt{\lambda^{2}(\tau)-\vec{\lambda}^{2}(\tau)}},
\end{aligned}
$$

the timelike worldline described by the origin of the Wigner hyperplane is arbitrary (i.e. gauge dependent): $x_{s}^{\mu}(\tau)$ may be any covariant noncanonical centroid [the real "external" center of mass is the canonical noncovariant $\tilde{x}_{s}^{\mu}\left(T_{s}\right)=x_{s}^{\mu}\left(T_{s}\right)-\frac{1}{\epsilon_{s}\left(p_{s}^{o}+\epsilon_{s}\right)}\left[p_{s \nu} S_{s}^{\nu \mu}+\epsilon_{s}\left(S_{s}^{o \mu}+\right.\right.$ $\left.\left.S_{s}^{o \nu} \frac{p_{s \nu} p_{s}^{\mu}}{\epsilon_{s}^{2}}\right)\right]$ : it describes a decoupled point particle observer ; see Section VI].

In the gauge $T_{s}-\tau \approx 0, \vec{X}_{\phi} \approx 0$, implying $\lambda(\tau)=-1, \vec{\lambda}(\tau)=0\left[g_{\tau \tau}=1, g_{\tau r}=0\right]$, we get $\dot{x}_{s}^{\mu}\left(T_{s}\right)=u^{\mu}\left(p_{s}\right)$. Therefore, in this gauge, we have the centroid $x_{s}^{\mu}\left(T_{s}\right)=x_{s}^{\left(\vec{X}_{\phi}\right) \mu}\left(T_{s}\right)=$ $x_{s}^{\mu}(0)+T_{s} u^{\mu}\left(p_{s}\right)$, which carries the Klein-Gordon "internal" collective variable $\vec{\sigma}_{\phi}=\vec{X}_{\phi} \approx 0$ (see Section VI).

In this gauge we get the following form of the energy-momentum tensor $\left[\eta^{\mu \nu}=\right.$ $\left.u^{\mu}\left(p_{s}\right) u^{\nu}\left(p_{s}\right)-\sum_{r=1}^{3} \epsilon_{r}^{\mu}\left(u\left(p_{s}\right)\right) \epsilon_{r}^{\nu}\left(u\left(p_{s}\right)\right)\right]$

$$
\begin{aligned}
T^{\mu \nu}\left[x_{s}^{\mu}\left(T_{s}\right)+\epsilon_{u}^{\mu}\left(u\left(p_{s}\right)\right) \sigma^{u}\right][\phi] & =T^{\mu \nu}\left[x_{s}^{\mu}\left(T_{s}\right)+\epsilon_{u}^{\mu}\left(u\left(p_{s}\right)\right) \sigma^{u}\right]\left[X_{\phi}^{A}, P_{\phi}^{A}, \mathbf{H}, \mathbf{K}\right]= \\
& =\frac{1}{2} u^{\mu}\left(p_{s}\right) u^{\nu}\left(p_{s}\right)\left[\pi^{2}+(\vec{\partial} \phi)^{2}+m^{2} \phi^{2}\right]\left(T_{s}, \vec{\sigma}\right)+ \\
& +\epsilon_{r}^{\mu}\left(u\left(p_{s}\right)\right) \epsilon_{s}^{\nu}\left(u\left(p_{s}\right)\right)\left[-\frac{1}{2} \delta_{r s}\left[\pi^{2}-(\vec{\partial} \phi)^{2}-m^{2} \phi^{2}\right]+\right. \\
& \left.+\partial_{r} \phi \partial_{s} \phi\right]\left(T_{s}, \vec{\sigma}\right)- \\
& -\left[u^{\mu}\left(p_{s}\right) \epsilon_{r}^{\nu}\left(u\left(p_{s}\right)\right)+u^{\nu}\left(p_{s}\right) \epsilon_{r}^{\mu}\left(u\left(p_{s}\right)\right)\right]\left[\pi \partial_{r} \phi\right]\left(T_{s}, \vec{\sigma}\right)= \\
& =\left[\rho[\phi, \pi] u^{\mu}\left(p_{s}\right) u^{\nu}\left(p_{s}\right)+\mathcal{P}[\phi, \pi]\left[\eta^{\mu \nu}-u^{\mu}\left(p_{s}\right) u^{\nu}\left(p_{s}\right)\right]+\right. \\
& +u^{\mu}\left(p_{s}\right) q^{\nu}[\phi, \pi]+u^{\nu}\left(p_{s}\right) q^{\mu}[\phi, \pi]+ \\
& \left.+T_{a n s}^{r s t r e s s}[\phi, \pi] \epsilon_{r}^{\mu}\left(u\left(p_{s}\right)\right) \epsilon_{s}^{\nu}\left(u\left(p_{s}\right)\right)\right]\left(T_{s}, \vec{\sigma}\right), \\
\rho[\phi, \pi] & =\frac{1}{2}\left[\pi^{2}+(\vec{\partial} \phi)^{2}+m^{2} \phi^{2}\right], \\
\mathcal{P}[\phi, \pi] & =\frac{1}{2}\left[\pi^{2}-\frac{5}{3}(\vec{\partial} \phi)^{2}-m^{2} \phi^{2}\right], \\
q^{\mu}[\phi, \pi] & =-\pi \partial_{r} \phi \epsilon_{r}^{\mu}\left(u\left(p_{s}\right)\right),
\end{aligned}
$$




$$
\begin{aligned}
T_{\text {an stress }}^{r s}[\phi, \pi]= & -\left[\partial^{r} \phi \partial^{s} \phi-\frac{1}{3} \delta^{r s}(\vec{\partial} \phi)^{2}\right], \\
& \delta_{u v} T_{\text {an stress }}^{u v}[\phi, \pi]=0, \\
T_{\text {stress }}^{r s}\left(T_{s}, \vec{\sigma}\right)[\phi]= & \epsilon_{\mu}^{r}\left(u\left(p_{s}\right)\right) \epsilon_{\nu}^{s}\left(u\left(p_{s}\right)\right) T^{\mu \nu}\left[x_{s}^{\mu}\left(T_{s}\right)+\epsilon_{u}^{\mu}\left(u\left(p_{s}\right)\right) \sigma^{u}\right][\phi]= \\
= & {\left[\partial^{r} \phi \partial^{s} \phi\right]\left(T_{s}, \vec{\sigma}\right)-\frac{1}{2} \delta^{r s}\left[\pi^{2}-(\vec{\partial} \phi)^{2}-m^{2} \phi^{2}\right]\left(T_{s}, \vec{\sigma}\right), } \\
T_{\mu}^{\mu}\left[x_{s}^{\mu}\left(T_{s}\right)+\epsilon_{u}^{\mu}\left(u\left(p_{s}\right)\right) \sigma^{u}\right][\phi]= & 2\left[\pi^{2}-(\vec{\partial} \phi)^{2}-m^{2} \phi^{2}\right]\left(T_{s}, \vec{\sigma}\right), \\
T^{\mu \nu}\left[x_{s}^{\mu}\left(T_{s}\right)+\epsilon_{u}^{\mu}\left(u\left(p_{s}\right)\right) \sigma^{u}\right][\phi] u_{\nu}\left(p_{s}\right)= & \frac{1}{2}\left[\pi^{2}+(\vec{\partial} \phi)^{2}+m^{2} \phi^{2}\right]\left(T_{s}, \vec{\sigma}\right) u^{\mu}\left(p_{s}\right)+ \\
+ & {\left[\pi \partial^{r} \phi\right]\left(T_{s}, \vec{\sigma}\right) \epsilon_{r}^{\mu}\left(u\left(p_{s}\right)\right), } \\
P_{T}^{\mu}[\phi]= & \int d^{3} \sigma T^{\mu \nu}\left[x_{s}^{\mu}\left(T_{s}\right)+\epsilon_{u}^{\mu}\left(u\left(p_{s}\right)\right) \sigma^{u}\right][\phi] u_{\nu}\left(p_{s}\right)= \\
= & P_{\phi}^{\tau} u^{\mu}\left(p_{s}\right)+P_{\phi}^{r} \epsilon_{r}^{\mu}\left(u\left(p_{s}\right)\right) \approx P_{\phi}^{\tau} u^{\mu}\left(p_{s}\right) \approx p_{s}^{\mu}, \\
M_{\phi}= & P_{T}^{\mu}[\phi] u_{\mu}\left(p_{s}\right)=P_{\phi}^{\tau} .
\end{aligned}
$$

While the stress tensor of the Klein-Gordon field on the Wigner hyperplanes is $T_{\text {stress }}^{r s}\left(T_{s}, \vec{\sigma}\right)[\phi]$, from the last line of the expression of the energy-momentum tensor we see that it acquires a form reminiscent of the energy-momentum tensor of an ideal relativistic fluid as seen from a local observer at rest (Eckart decomposition; see Ref. [14] ): i) the constant normal $u^{\mu}\left(p_{s}\right)$ to the Wigner hyperplanes replaces the hydrodynamic velocity field of the fluid; ii) $\rho[\phi, \pi]\left(T_{s}, \vec{\sigma}\right]$ is the energy density; iii) $\mathcal{P}[\phi, \pi]\left(T_{s}, \vec{\sigma}\right)$ is the analogue of the pressure (sum of the thermodynamical pressure and of the non-equilibrium bulk stress or viscous pressure); iv) $q^{\mu}[\phi, \pi]\left(T_{s}, \vec{\sigma}\right)$ is the analogue of the heat flow; v) $T_{\text {an stress }}^{r s}[\phi, \pi]\left(T_{s}, \vec{\sigma}\right)$ is the shear (or anisotropic) stress tensor.

We can now study the manifestly Lorentz covariant Dixon multipoles [9] for the free real Klein-Gordon field on the Wigner hyperplanes in the gauge $\lambda(\tau)=-1, \vec{\lambda}(\tau)=0$ [so that $\dot{x}_{s}^{\mu}\left(T_{s}\right)=u^{\mu}\left(p_{s}\right), \ddot{x}_{s}^{\mu}\left(T_{s}\right)=0, x_{s}^{\mu}\left(T_{s}\right)=u^{\mu}\left(p_{s}\right) T_{s}+x_{s}^{\mu}(0)$ ] with respect to the origin $x_{s}^{\mu}\left(T_{s}\right)\left[\delta x_{s}^{\mu}(\vec{\sigma})=\epsilon_{u}^{\mu}\left(u\left(p_{s}\right)\right) \sigma^{u} ;\left(\mu_{1} . . \mu_{n}\right)\right.$ means symmetrization, while $\left[\mu_{1} . . \mu_{n}\right]$ means antisymmetrization]

$$
\begin{aligned}
t_{T}^{\mu_{1} \ldots \mu_{n} \mu \nu}\left(T_{s}\right)= & t_{T}^{\left(\mu_{1} \ldots \mu_{n}\right)(\mu \nu)}\left(T_{s}\right)= \\
= & \int d^{3} \sigma \delta x_{s}^{\mu_{1}}(\vec{\sigma}) \ldots \delta x_{s}^{\mu_{n}}(\vec{\sigma}) T^{\mu \nu}\left[x_{s}^{\mu}\left(T_{s}\right)+\epsilon_{u}^{\mu}\left(u\left(p_{s}\right)\right) \sigma^{u}\right][\phi]= \\
= & \epsilon_{r_{1}}^{\mu_{1}}\left(u\left(p_{s}\right)\right) \ldots \epsilon_{r_{n}}^{\mu_{n}}\left(u\left(p_{s}\right)\right) \\
& {\left[u^{\mu}\left(p_{s}\right) u^{\nu}\left(p_{s}\right) \frac{1}{2} \int d^{3} \sigma \sigma^{r_{1}} \ldots \sigma^{r_{n}}\left[\pi^{2}+(\vec{\partial} \phi)^{2}+m^{2} \phi^{2}\right]\left(T_{s}, \vec{\sigma}\right)+\right.} \\
+ & \epsilon_{r}^{\mu}\left(u\left(p_{s}\right)\right) \epsilon_{s}^{\nu}\left(u\left(p_{s}\right)\right) \int d^{3} \sigma \sigma^{r_{1}} \ldots \sigma^{r_{n}}\left[-\frac{1}{2} \delta_{r s}\left[\pi^{2}-(\vec{\partial} \phi)^{2}-m^{2} \phi^{2}\right]+\right. \\
+ & \left.\partial_{r} \phi \partial_{s} \phi\right]\left(T_{s}, \vec{\sigma}\right)+ \\
+ & {\left.\left[u^{\mu}\left(p_{s}\right) \epsilon_{r}^{\nu}\left(u\left(p_{s}\right)\right)+u^{\nu}\left(p_{s}\right) \epsilon_{r}^{\mu}\left(u\left(p_{s}\right)\right)\right] \int d^{3} \sigma \sigma^{r_{1}} \ldots \sigma^{r_{n}}\left[\pi \partial^{r} \phi\right]\left(T_{s}, \vec{\sigma}\right)\right]=}
\end{aligned}
$$




$$
\begin{aligned}
& =\epsilon_{r_{1}}^{\mu_{1}}\left(u\left(p_{s}\right)\right) \ldots \epsilon_{r_{n}}^{\mu_{n}}\left(u\left(p_{s}\right)\right) \epsilon_{A}^{\mu}\left(u\left(p_{s}\right)\right) \epsilon_{B}^{\nu}\left(u\left(p_{s}\right)\right) I_{T}^{r_{1} . . r_{n} A B}\left(T_{s}\right)= \\
& =\epsilon_{r_{1}}^{\mu_{1}}\left(u\left(p_{s}\right)\right) \ldots \epsilon_{r_{n}}^{\mu_{n}}\left(u\left(p_{s}\right)\right)\left[u^{\mu}\left(p_{s}\right) u^{\nu}\left(p_{s}\right) I_{T}^{r_{1} \ldots r_{n} \tau \tau}\left(T_{s}\right)+\right. \\
& +\epsilon_{r}^{\mu}\left(u\left(p_{s}\right)\right) \epsilon_{s}^{\nu}\left(u\left(p_{s}\right)\right) I_{T}^{r_{1} \ldots r_{n} r s}\left(T_{s}\right)+ \\
& \left.+\left[u^{\mu}\left(p_{s}\right) \epsilon_{r}^{\nu}\left(u\left(p_{s}\right)\right)+u^{\nu}\left(p_{s}\right) \epsilon_{r}^{\mu}\left(u\left(p_{s}\right)\right)\right] I_{T}^{r_{1} \ldots r_{n} r \tau}\left(T_{s}\right)\right]
\end{aligned}
$$

$$
\begin{aligned}
& u_{\mu_{1}}\left(p_{s}\right) \quad t_{T}^{\mu_{1} \ldots \mu_{n} \mu \nu}\left(T_{s}\right)=0 \\
& \text { For } \quad n=0 \text { (monopole) } \quad I_{T}^{\tau \tau}\left(T_{s}\right)=P_{\phi}^{\tau}, \quad I_{T}^{r \tau}\left(T_{s}\right)=P_{\phi}^{r} \text {, } \\
& t_{T}^{\mu_{1} \ldots \mu_{n} \mu}{ }_{\mu}\left(T_{s}\right)=\int d^{3} \sigma \delta x_{s}^{\mu_{1}}(\vec{\sigma}) \ldots \delta x_{s}^{\mu_{n}}(\vec{\sigma}) T_{\mu}^{\mu}\left[x_{s}^{\mu}\left(T_{s}\right)+\epsilon_{u}^{\mu}\left(u\left(p_{s}\right)\right) \sigma^{u}\right][\phi]= \\
& =\epsilon_{r_{1}}^{\mu_{1}}\left(u\left(p_{s}\right)\right) \ldots \epsilon_{r_{n}}^{\mu_{n}}\left(u\left(p_{s}\right)\right) \\
& \int d^{3} \sigma \sigma^{r_{1}} \ldots \sigma^{r_{n}}\left[2\left[\pi^{2}-(\vec{\partial} \phi)^{2}\right]-m^{2} \phi^{2}\right]\left(T_{s}, \vec{\sigma}\right)= \\
& \stackrel{\text { def }}{=} \epsilon_{r_{1}}^{\mu_{1}}\left(u\left(p_{s}\right)\right) \ldots \epsilon_{r_{n}}^{\mu_{n}}\left(u\left(p_{s}\right)\right) I_{T}^{r_{1} \ldots r_{n} A}{ }_{A}\left(T_{s}\right) \\
& I_{T}^{r_{1} r_{2} A}{ }_{A}\left(T_{s}\right)=\check{I}_{T}^{r_{1} r_{2} A}{ }_{A}\left(T_{s}\right)-\frac{1}{3} \delta^{r_{1} r_{2}} \delta_{u v} I_{T}^{u v A}{ }_{A}\left(T_{s}\right)=i_{T}^{r_{1} r_{2}}\left(T_{s}\right)-\frac{1}{2} \delta^{r_{1} r_{2}} \delta_{u v} i_{T}^{u v}\left(T_{s}\right), \\
& \check{I}_{T}^{r_{1} r_{2} A}{ }_{A}\left(T_{s}\right)=i_{T}^{r_{1} r_{2}}\left(T_{s}\right)-\frac{1}{3} \delta^{r_{1} r_{2}} \delta_{u v} i_{T}^{u v}\left(T_{s}\right), \quad \delta_{u v} \check{I}_{T}^{u v A}{ }_{A}\left(T_{s}\right)=0, \\
& i_{T}^{r_{1} r_{2}}\left(T_{s}\right)=I_{T}^{r_{1} r_{2} A}{ }_{A}\left(T_{s}\right)-\delta^{r_{1} r_{2}} \delta_{u v} I_{T}^{u v A}{ }_{A}\left(T_{s}\right)= \\
& =\int d^{3} \sigma\left(\sigma^{r_{1}} \sigma^{r_{2}}-\delta^{r_{1} r_{2}} \vec{\sigma}^{2}\right)\left[2\left[\pi^{2}-(\vec{\partial} \phi)^{2}\right]-m^{2} \phi^{2}\right]\left(T_{s}, \vec{\sigma}\right), \\
& \tilde{t}_{T}^{\mu_{1} \ldots \mu_{n}}\left(T_{s}\right)=t_{T}^{\mu_{1} \ldots \mu_{n} \mu \nu}\left(T_{s}\right) u_{\mu}\left(p_{s}\right) u_{\nu}\left(p_{s}\right)= \\
& =\epsilon_{r_{1}}^{\mu_{1}}\left(u\left(p_{s}\right)\right) \ldots \epsilon_{r_{n}}^{\mu_{n}}\left(u\left(p_{s}\right)\right) I_{T}^{r_{1} . . r_{n} \tau \tau}\left(T_{s}\right), \\
& \tilde{t}_{T}^{\mu_{1}}\left(T_{s}\right)=\epsilon_{r_{1}}^{\mu_{1}}\left(u\left(p_{s}\right)\right) \frac{1}{2} \int d^{3} \sigma \sigma^{r_{1}}\left[\pi^{2}+(\vec{\partial} \phi)^{2}+m^{2} \phi^{2}\right]\left(T_{s}, \vec{\sigma}\right)= \\
& =\epsilon_{r_{1}}^{\mu_{1}}\left(u\left(p_{s}\right)\right) I_{T}^{r_{1} \tau \tau}\left(T_{s}\right)=-P_{\phi}^{\tau} \epsilon_{r_{1}}^{\mu_{1}}\left(u\left(p_{s}\right)\right) r_{\phi}^{r_{1}}, \\
& \tilde{t}_{T}^{\mu_{1} \mu_{2}}\left(T_{s}\right)=\epsilon_{r_{1}}^{\mu_{1}}\left(u\left(p_{s}\right)\right) \epsilon_{r_{2}}^{\mu_{2}}\left(u\left(p_{s}\right)\right) I_{T}^{r_{1} r_{2} \tau \tau}\left(T_{s}\right) \\
& I_{T}^{r_{1} r_{2} \tau \tau}\left(T_{s}\right)=\hat{I}_{T}^{r_{1} r_{2} \tau \tau}\left(T_{s}\right)-\frac{1}{3} \delta^{r_{1} r_{2}} \delta_{u v} I_{T}^{u v \tau \tau}\left(T_{s}\right)=\tilde{i}_{T}^{r_{1} r_{2}}\left(T_{s}\right)-\frac{1}{2} \delta^{r_{1} r_{2}} \delta_{u v} \tilde{i}_{T}^{u v}\left(T_{s}\right), \\
& \hat{I}_{T}^{r_{1} r_{2} \tau \tau}\left(T_{s}\right)=\tilde{i}_{T}^{r_{1} r_{2}}\left(T_{s}\right)-\frac{1}{3} \delta^{r_{1} r_{2}} \delta_{u v} \tilde{i}_{T}^{u v}\left(T_{s}\right), \quad \delta_{u v} \hat{I}_{T}^{u v \tau \tau}\left(T_{s}\right)=0, \\
& \tilde{i}_{T}^{r_{1} r_{2}}\left(T_{s}\right)=I_{T}^{r_{1} r_{2} \tau \tau}\left(T_{s}\right)-\delta^{r_{1} r_{2}} \delta_{u v} I_{T}^{u v \tau \tau}\left(T_{s}\right) .
\end{aligned}
$$

The Wigner covariant multipoles $I_{T}^{r_{1} . . r_{n} \tau \tau}\left(T_{s}\right), I_{T}^{r_{1} . . r_{n} r s}\left(T_{s}\right), I_{T}^{r_{1} . . r_{n} r \tau}\left(T_{s}\right)$ are the mass, stress and momentum multipoles respectively.

The quantities $\check{I}_{T}^{r_{1} r_{2} A}{ }_{A}\left(T_{s}\right)$ and $i_{T}^{r_{1} r_{2}}\left(T_{s}\right)$ are the traceless quadrupole moment and the inertia tensor defined by Thorne in Ref. 15.

The quantities $I_{T}^{r_{1} r_{2} \tau \tau}\left(T_{s}\right)$ and $\tilde{i}_{T}^{r_{1} r_{2}}\left(T_{s}\right)$ are Dixon's definitions of quadrupole moment 
and of tensor of inertia respectively.

Moreover, Dixon's definition of "center of mass" of an extended object is $\tilde{t}_{T}^{\mu_{1}}\left(T_{s}\right)=0$ or $I_{T}^{r \tau \tau}\left(T_{s}\right)=-P_{\phi}^{\tau} r_{\phi}^{r}=0$ : therefore the quantity $\vec{r}_{\phi}$ defined in the previous equation is a noncanonical $\left[\left\{r_{\phi}^{r}, r_{\phi}^{s}\right\}=S_{\phi}^{r s}\right]$ candidate for the "internal" center of mass of the field configuration: its vanishing is a gauge fixing for $\vec{P}_{\phi} \approx 0$ and implies $x_{s}^{\mu}\left(T_{s}\right)=x_{s}^{\left(\vec{r}_{\phi}\right) \mu}\left(T_{s}\right)=$ $x_{s}^{\mu}(0)+u^{\mu}\left(p_{s}\right) T_{s}$ (see next Section).

When $I_{T}^{r \tau \tau}\left(T_{s}\right)=0$, the equations $0=\frac{d I_{T}^{r \tau \tau}\left(T_{s}\right)}{d T_{s}}=-P_{\phi}^{\tau} \frac{d r_{\phi}^{r}}{d T_{s}}=\frac{d}{d T_{s}} \int d^{3} \sigma \sigma^{r}\left[\pi^{2}+(\vec{\partial} \phi)^{2}+\right.$ $\left.m^{2} \phi^{2}\right]\left(T_{s}, \vec{\sigma}\right) \stackrel{\circ}{=}-P_{\phi}^{r}$ implies the correct momentum-velocity relation $\frac{\vec{P}_{\phi}}{P_{\phi}^{\tau}} \stackrel{\circ}{=} \frac{d \vec{r}_{\phi}}{d T_{s}} \approx 0 \dot{i}$

These multipoles, whose Poisson brackets are non-trivial, are related to the previous ones of Eqs.(4.41), with the Poisson brackets given in Eqs.(4.42), in the following way (remember that these other multipoles exist only if the Klein-Gordon fields have compact support V in momentum space)

$$
\begin{aligned}
& I_{T}^{r_{1} \ldots r_{n} \tau \tau}\left(T_{s}\right)=\frac{1}{2} \int d^{3} \sigma \sigma^{r_{1}} \ldots \sigma^{r_{n}}\left[\pi^{2}+(\vec{\partial} \phi)^{2}+m^{2} \phi^{2}\right]\left(T_{s}, \vec{\sigma}\right)= \\
& =2 \int d \tilde{q}_{1} d \tilde{q}_{2} \mathbf{A}_{\vec{q}_{1}}\left(T_{s} ; P_{\phi}^{\tau}, \mathbf{H}\right] \mathbf{A}_{\vec{q}_{2}}\left(T_{s} ; P_{\phi}^{\tau}, \mathbf{H}\right] \int d^{3} \sigma \sigma^{r_{1}} \ldots \sigma^{r_{n}} \\
& \left(\left[\omega\left(q_{1}\right) \omega\left(q_{2}\right)+\vec{q}_{1} \cdot \vec{q}_{2}\right] \sin \left(\vec{q}_{1} \cdot \vec{\sigma}+\mathbf{B}_{\vec{q}_{1}}\left(T_{s} ; \tilde{X}_{\phi}^{\tau}, \mathbf{K}\right]\right) \sin \left(\vec{q}_{2} \cdot \vec{\sigma}+\mathbf{B}_{\vec{q}_{2}}\left(T_{s} ; \tilde{X}_{\phi}^{\tau}, \mathbf{K}\right]\right)+\right. \\
& \left.+m^{2} \cos \left(\vec{q}_{1} \cdot \vec{\sigma}+\mathbf{B}_{\vec{q}_{1}}\left(T_{s} ; \tilde{X}_{\phi}^{\tau}, \mathbf{K}\right]\right) \cos \left(\vec{q}_{2} \cdot \vec{\sigma}+\mathbf{B}_{\vec{q}_{2}}\left(T_{s} ; \tilde{X}_{\phi}^{\tau}, \mathbf{K}\right]\right)\right)= \\
& =\frac{1}{2} \sum_{h, k=0}^{\infty}(-)^{h+k} \\
& \left(\frac { 1 } { ( 2 h + 1 ) ! ( 2 k + 1 ) ! } \left[V_{1}^{r_{1} \ldots r_{n} u_{1} \ldots u_{2 h+1} v_{1} \ldots v_{2 k+1}}\right.\right. \\
& {\left[\mathcal{T}_{\pi, O}^{u_{1} \ldots u_{2 h+1}} \mathcal{T}_{\pi, O}^{v_{1} \ldots v_{2 k+1}}+m^{2} \mathcal{T}_{\phi, O}^{u_{1} \ldots u_{2 h+1}} \mathcal{T}_{\phi, O}^{v_{1} \ldots v_{2 k+1}}\right]+} \\
& \left.+V_{2}^{r_{1} \ldots r_{n} u_{1} \ldots u_{2 h+1} v_{1} \ldots v_{2 k+1}} \mathcal{T}_{\phi, O}^{u_{1} \ldots u_{2 h+1}} \mathcal{T}_{\phi, O}^{v_{1} \ldots v_{2 k+1}}\right]- \\
& -\frac{1}{(2 h+1) !(2 k) !}\left[V_{1}^{r_{1} \ldots r_{n} u_{1} \ldots u_{2 h+1} v_{1} \ldots v_{2 k}}\left[\mathcal{T}_{\pi, O}^{u_{1} \ldots u_{2 h+1}} \mathcal{T}_{\pi, E}^{v_{1} \ldots v_{2 k}}+m^{2} \mathcal{T}_{\phi, O}^{u_{1} \ldots u_{2 h+1}} \mathcal{T}_{\phi, E}^{v_{1} \ldots v_{2 k}}\right]+\right. \\
& \left.+V_{2}^{r_{1} \ldots r_{n} u_{1} \ldots u_{2 h+1} v_{1} \ldots v_{2 k}} \mathcal{T}_{\phi, O}^{u_{1} \ldots u_{2 h+1}} \mathcal{T}_{\phi, E}^{v_{1} \ldots v_{2 k}}\right]- \\
& -\frac{1}{(2 h) !(2 k+1) !}\left[V_{1}^{r_{1} \ldots r_{n} u_{1} \ldots u_{2 h} v_{1} \ldots v_{2 k+1}}\left[\mathcal{T}_{\pi, E}^{u_{1} \ldots u_{2 h}} \mathcal{T}_{\pi, O}^{v_{1} \ldots v_{2 k+1}}+m^{2} \mathcal{T}_{\phi, E}^{u_{1} \ldots u_{2 h}} \mathcal{T}_{\phi, O}^{v_{1} \ldots v_{2 k+1}}\right]+\right. \\
& \left.+V_{2}^{r_{1} \ldots r_{n} u_{1} \ldots u_{2 h} v_{1} \ldots v_{2 k+1}} \mathcal{T}_{\phi, E}^{u_{1} \ldots u_{2 h}} \mathcal{T}_{\phi, O}^{v_{1} \ldots v_{2 k+1}}\right]+ \\
& +\frac{1}{(2 h) !(2 k) !}\left[V_{1}^{r_{1} \ldots r_{n} u_{1} \ldots u_{2 h} v_{1} \ldots v_{2 k}}\left[\mathcal{T}_{\pi, E}^{u_{1} \ldots u_{2 h}} \mathcal{T}_{\pi, E}^{v_{1} \ldots v_{2 k}}+m^{2} \mathcal{T}_{\phi, E}^{u_{1} \ldots u_{2 h}} \mathcal{T}_{\phi, E}^{v_{1} \ldots v_{2 k}}\right]+\right. \\
& \left.\left.+V_{2}^{r_{1} \ldots r_{n} u_{1} \ldots u_{2 h} v_{1} \ldots v_{2 k}} \mathcal{T}_{\phi, E}^{u_{1} \ldots u_{2 h}} \mathcal{T}_{\phi, E}^{v_{1} \ldots v_{2 k}}\right]\right) \\
& I_{T}^{r_{1} \ldots r_{n} r s}\left(T_{s}\right)=\int d^{3} \sigma \sigma^{r_{1}} \ldots \sigma^{r_{n}}\left[-\frac{1}{2} \delta_{r s}\left[\pi^{2}-(\vec{\partial} \phi)^{2}-m^{2} \phi^{2}\right]+\partial_{r} \phi \partial_{s} \phi\right]\left(T_{s}, \vec{\sigma}\right)= \\
& =4 \int d \tilde{q}_{1} d \tilde{q}_{2} \mathbf{A}_{\vec{q}_{1}}\left(T_{s} ; P_{\phi}^{\tau}, \mathbf{H}\right] \mathbf{A}_{\vec{q}_{2}}\left(T_{s} ; P_{\phi}^{\tau}, \mathbf{H}\right] \int d^{3} \sigma \sigma^{r_{1}} \ldots \sigma^{r_{n}} \\
& \left(-\frac{1}{2} \delta^{r s}\left[\left[\omega\left(q_{1}\right) \omega\left(q_{2}\right)-\overrightarrow{q_{1}} \cdot \overrightarrow{q_{2}}\right]\right.\right. \\
& \sin \left(\vec{q}_{1} \cdot \vec{\sigma}+\mathbf{B}_{\vec{q}_{1}}\left(T_{s} ; \tilde{X}_{\phi}^{\tau}, \mathbf{K}\right]\right) \sin \left(\overrightarrow{q_{2}} \cdot \vec{\sigma}+\mathbf{B}_{\vec{q}_{2}}\left(T_{s} ; \tilde{X}_{\phi}^{\tau}, \mathbf{K}\right]\right)-
\end{aligned}
$$




$$
\begin{aligned}
& \left.-m^{2} \cos \left(\vec{q}_{1} \cdot \vec{\sigma}+\mathbf{B}_{\vec{q}_{1}}\left(T_{s} ; \tilde{X}_{\phi}^{\tau}, \mathbf{K}\right]\right) \cos \left(\vec{q}_{2} \cdot \vec{\sigma}+\mathbf{B}_{\vec{q}_{2}}\left(T_{s} ; \tilde{X}_{\phi}^{\tau}, \mathbf{K}\right]\right)\right]+ \\
& \left.+q_{1}^{r} q_{2}^{s} \sin \left(\vec{q}_{1} \cdot \vec{\sigma}+\mathbf{B}_{\vec{q}_{1}}\left(T_{s} ; \tilde{X}_{\phi}^{\tau}, \mathbf{K}\right]\right) \sin \left(\vec{q}_{2} \cdot \vec{\sigma}+\mathbf{B}_{\vec{q}_{2}}\left(T_{s} ; \tilde{X}_{\phi}^{\tau}, \mathbf{K}\right]\right)\right)= \\
& =\sum_{h, k=0}^{\infty}(-)^{h+k} \\
& \left(\frac { 1 } { ( 2 h + 1 ) ! ( 2 k + 1 ) ! } \left[-\frac{1}{2} \delta^{r s} V_{1}^{r_{1} \ldots r_{n} u_{1} \ldots u_{2 h+1} v_{1} \ldots v_{2 k+1}}\right.\right. \\
& {\left[\mathcal{T}_{\pi, O}^{u_{1} \ldots u_{2 h+1}} \mathcal{T}_{\pi, O}^{v_{1} \ldots v_{2 k+1}}-m^{2} \mathcal{T}_{\phi, O}^{u_{1} \ldots u_{2 h+1}} \mathcal{T}_{\phi, O}^{v_{1} \ldots v_{2 k+1}}\right]+} \\
& \left.+\left(\frac{1}{2} \delta^{r s} V_{2}^{r_{1} \ldots r_{n} u_{1} \ldots u_{2 h+1} v_{1} \ldots v_{2 k+1}}+V_{3}^{r_{1} \ldots r_{n} u_{1} \ldots u_{2 h+1} v_{1} \ldots v_{2 k+1} r s}\right) \mathcal{T}_{\phi, O}^{u_{1} \ldots u_{2 h+1}} \mathcal{T}_{\phi, O}^{v_{1} \ldots v_{2 k+1}}\right]- \\
& -\frac{1}{(2 h+1) !(2 k) !}\left[-\frac{1}{2} \delta^{r s} V_{1}^{r_{1} \ldots r_{n} u_{1} \ldots u_{2 h+1} v_{1} \ldots v_{2 k}}\right. \\
& {\left[\mathcal{T}_{\pi, O}^{u_{1} \ldots u_{2 h+1}} \mathcal{T}_{\pi, E}^{v_{1} \ldots v_{2 k}}-m^{2} \mathcal{T}_{\phi, O}^{u_{1} \ldots u_{2 h+1}} \mathcal{T}_{\phi, E}^{v_{1} \ldots v_{2 k}}\right]+} \\
& \left.+\left(\frac{1}{2} \delta^{r s} V_{2}^{r_{1} \ldots r_{n} u_{1} \ldots u_{2 h+1} v_{1} \ldots v_{2 k}}+V_{3}^{r_{1} \ldots r_{n} u_{1} \ldots u_{2 h+1} v_{1} \ldots v_{2 k} r s}\right) \mathcal{T}_{\phi, O}^{u_{1} \ldots u_{2 h+1}} \mathcal{T}_{\phi, E}^{v_{1} \ldots v_{2 k}}\right]- \\
& -\frac{1}{(2 h) !(2 k+1) !}\left[-\frac{1}{2} \delta^{r s} V_{1}^{r_{1} \ldots r_{n} u_{1} \ldots u_{2 h} v_{1} \ldots v_{2 k+1}}\right. \\
& {\left[\mathcal{T}_{\pi, E}^{u_{1} \ldots u_{2 h}} \mathcal{T}_{\pi, O}^{v_{1} \ldots v_{2 k+1}}-m^{2} \mathcal{T}_{\phi, E}^{u_{1} \ldots u_{2 h}} \mathcal{T}_{\phi, O}^{v_{1} \ldots v_{2 k+1}}\right]+} \\
& \left.+\left(\frac{1}{2} \delta^{r s} V_{2}^{r_{1} \ldots r_{n} u_{1} \ldots u_{2 h} v_{1} \ldots v_{2 k+1}}+V_{3}^{r_{1} \ldots r_{n} u_{1} \ldots u_{2 h} v_{1} \ldots v_{2 k+1} r s}\right) \mathcal{T}_{\phi, E}^{u_{1} \ldots u_{2 h}} \mathcal{T}_{\phi, O}^{v_{1} \ldots v_{2 k+1}}\right]+ \\
& +\frac{1}{(2 h) !(2 k) !}\left[-\frac{1}{2} \delta^{r s} V_{1}^{r_{1} \ldots r_{n} u_{1} \ldots u_{2 h} v_{1} \ldots v_{2 k}}\left[\mathcal{T}_{\pi, E}^{u_{1} \ldots u_{2 h}} \mathcal{T}_{\pi, E}^{v_{1} \ldots v_{2 k}}-m^{2} \mathcal{T}_{\phi, E}^{u_{1} \ldots u_{2 h}} \mathcal{T}_{\phi, E}^{v_{1} \ldots v_{2 k}}\right]+\right. \\
& \left.\left.+\left(\frac{1}{2} \delta^{r s} V_{2}^{r_{1} \ldots r_{n} u_{1} \ldots u_{2 h} v_{1} \ldots v_{2 k}}+V_{3}^{r_{1} \ldots r_{n} u_{1} \ldots u_{2 h} v_{1} \ldots v_{2 k} r s}\right) \mathcal{T}_{\phi, E}^{u_{1} \ldots u_{2 h}} \mathcal{T}_{\phi, E}^{v_{1} \ldots v_{2 k}}\right]\right), \\
& I_{T}^{r_{1} \ldots r_{n} r \tau}\left(T_{s}\right)=\int d^{3} \sigma \sigma^{r_{1}} \ldots \sigma^{r_{n}}\left[\pi \partial^{r} \phi\right]\left(T_{s}, \vec{\sigma}\right)= \\
& =4 \int d \tilde{q}_{1} d \tilde{q}_{2} \mathbf{A}_{\vec{q}_{1}}\left(T_{s} ; P_{\phi}^{\tau}, \mathbf{H}\right] \mathbf{A}_{\vec{q}_{2}}\left(T_{s} ; P_{\phi}^{\tau}, \mathbf{H}\right] \omega\left(q_{1}\right) q_{2}^{r} \int d^{3} \sigma \sigma^{r_{1}} \ldots \sigma^{r_{n}} \\
& \sin \left(\vec{q}_{1} \cdot \vec{\sigma}+\mathbf{B}_{\vec{q}_{1}}\left(T_{s} ; \tilde{X}_{\phi}^{\tau}, \mathbf{K}\right]\right) \sin \left(\vec{q}_{2} \cdot \vec{\sigma}+\mathbf{B}_{\vec{q}_{2}}\left(T_{s} ; \tilde{X}_{\phi}^{\tau}, \mathbf{K}\right]\right)= \\
& =\sum_{h, k=0}^{\infty}(-)^{h+k} \\
& \left(\frac{1}{(2 h+1) !(2 k+1) !} V_{4}^{r_{1} \ldots r_{n} u_{1} \ldots u_{2 h+1} v_{1} \ldots v_{2 k+1}} \mathcal{T}_{\pi, O}^{u_{1} \ldots u_{2 h+1}} \mathcal{T}_{\phi, O}^{v_{1} \ldots v_{2 k+1}}-\right. \\
& -\frac{1}{(2 h+1) !(2 k) !} V_{4}^{r_{1} \ldots r_{n} u_{1} \ldots u_{2 h+1} v_{1} \ldots v_{2 k}} \mathcal{T}_{\pi, O}^{u_{1} \ldots u_{2 h+1}} \mathcal{T}_{\phi, E}^{v_{1} \ldots v_{2 k}}- \\
& -\frac{1}{(2 h) !(2 k+1) !} V_{4}^{r_{1} \ldots r_{n} u_{1} \ldots u_{2 h} v_{1} \ldots v_{2 k+1}} \mathcal{T}_{\pi, E}^{u_{1} \ldots u_{2 h}} \mathcal{T}_{\phi, O}^{v_{1} \ldots v_{2 k+1}}+ \\
& \left.+\frac{1}{(2 h) !(2 k) !} V_{4}^{r_{1} \ldots r_{n} u_{1} \ldots u_{2 h} v_{1} \ldots v_{2 k}} \mathcal{T}_{\pi, E}^{u_{1} \ldots u_{2 h}} \mathcal{T}_{\phi, E}^{v_{1} \ldots v_{2 k}}\right)
\end{aligned}
$$




$$
\begin{aligned}
& V_{1}^{r_{1} \ldots r_{n} u_{1} \ldots u_{h} v_{1} \ldots v_{k}}=\int_{V} d^{3} \sigma \sigma^{r_{1}} \ldots \sigma^{r_{n}} \sigma^{u_{1}} \ldots \sigma^{u_{h}} \sigma^{v_{1}} \ldots \sigma^{v_{k}} \\
& V_{2}^{r_{1} \ldots r_{n} u_{1} \ldots u_{h} v_{1} \ldots v_{k}}=\int_{V} d^{3} \sigma \sigma^{r_{1}} \ldots \sigma^{r_{n}} \vec{\partial}\left(\sigma^{u_{1}} \ldots \sigma^{u_{h}}\right) \cdot \vec{\partial}\left(\sigma^{v_{1}} \ldots \sigma^{v_{k}}\right), \\
& V_{3}^{r_{1} \ldots r_{n} u_{1} \ldots u_{h} v_{1} \ldots v_{k} r s}=\int_{V} d^{3} \sigma \sigma^{r_{1}} \ldots \sigma^{r_{n}} \partial^{r}\left(\sigma^{u_{1}} \ldots \sigma^{u_{h}}\right) \partial^{s}\left(\sigma^{v_{1}} \ldots \sigma^{v_{k}}\right), \\
& V_{4}^{r_{1} \ldots r_{n} u_{1} \ldots u_{h} v_{1} \ldots v_{k} r}=\int_{V} d^{3} \sigma \sigma^{r_{1}} \ldots \sigma^{r_{n}} \sigma^{u_{1}} \ldots \sigma^{u_{h}} \partial^{r}\left(\sigma^{v_{1}} \ldots \sigma^{v_{k}}\right) .
\end{aligned}
$$

Then there are the related Dixon multipoles

$$
\begin{aligned}
p_{T}^{\mu_{1} \ldots \mu_{n} \mu}\left(T_{s}\right)= & t_{T}^{\mu_{1} \ldots \mu_{n} \mu \nu}\left(T_{s}\right) u_{\nu}\left(p_{s}\right)=p_{T}^{\left(\mu_{1} \ldots \mu_{n}\right) \mu}\left(T_{s}\right)= \\
= & \epsilon_{r_{1}}^{\mu_{1}}\left(u\left(p_{s}\right)\right) \ldots \epsilon_{r_{n}}^{\mu_{n}}\left(u\left(p_{s}\right)\right) \epsilon_{A}^{\mu}\left(u\left(p_{s}\right)\right) I_{T}^{r_{1} . . r_{n} A \tau}\left(T_{s}\right)= \\
= & \epsilon_{r_{1}}^{\mu_{1}}\left(u\left(p_{s}\right)\right) \ldots \epsilon_{r_{n}}^{\mu_{n}}\left(u\left(p_{s}\right)\right) \int d^{3} \sigma \sigma^{r_{1}} \ldots \sigma^{r_{n}}\left[\frac{1}{2}\left[\pi^{2}+(\vec{\partial} \phi)^{2}+m^{2} \phi^{2}\right]\left(T_{s}, \vec{\sigma}\right) u^{\mu}\left(p_{s}\right)+\right. \\
+ & {\left.\left[\pi \partial^{r} \phi\right]\left(T_{s}, \vec{\sigma}\right) \epsilon_{r}^{\mu}\left(u\left(p_{s}\right)\right)\right]=} \\
= & 2 \epsilon_{r_{1}}^{\mu_{1}}\left(u\left(p_{s}\right)\right) \ldots \epsilon_{r_{n}}^{\mu_{n}}\left(u\left(p_{s}\right)\right) \\
& \int d \tilde{q}_{1} d \tilde{q}_{2} \mathbf{A}_{\vec{q}_{1}}\left(T_{s} ; P_{\phi}^{\tau}, \mathbf{H}\right] \mathbf{A}_{\vec{q}_{2}}\left(T_{s} ; P_{\phi}^{\tau}, \mathbf{H}\right] \int d^{3} \sigma \sigma^{r_{1}} \ldots \sigma^{r_{n}} \\
& {\left[u ^ { \mu } ( p _ { s } ) \left(\left(\omega\left(q_{1}\right) \omega\left(q_{2}\right)+\vec{q}_{1} \cdot \vec{q}_{2}\right)\right.\right.} \\
& s i n\left(\vec{q}_{1} \cdot \vec{\sigma}+\mathbf{B}_{\vec{q}_{1}}\left(T_{s} ; \tilde{X}_{\phi}^{\tau}, \mathbf{K}\right]\right) \sin \left(\overrightarrow{q_{2}} \cdot \vec{\sigma}+\mathbf{B}_{\vec{q}_{2}}\left(T_{s} ; \tilde{X}_{\phi}^{\tau}, \mathbf{K}\right]\right)+ \\
+ & \left.m^{2} \cos \left(\vec{q}_{1} \cdot \vec{\sigma}+\mathbf{B}_{\vec{q}_{1}}\left(T_{s} ; \tilde{X}_{\phi}^{\tau}, \mathbf{K}\right]\right) \cos \left(\vec{q}_{1} \cdot \vec{\sigma}+\mathbf{B}_{\vec{q}_{1}}\left(T_{s} ; \tilde{X}_{\phi}^{\tau}, \mathbf{K}\right]\right)\right)- \\
- & \left.\epsilon_{r}^{\mu}\left(u\left(p_{s}\right)\right) q_{2}^{r} \sin \left(\vec{q}_{1} \cdot \vec{\sigma}+\mathbf{B}_{\vec{q}_{1}}\left(T_{s} ; \tilde{X}_{\phi}^{\tau}, \mathbf{K}\right]\right) \sin \left(\vec{q}_{2} \cdot \vec{\sigma}+\mathbf{B}_{\vec{q}_{2}}\left(T_{s} ; \tilde{X}_{\phi}^{\tau}, \mathbf{K}\right]\right)\right], \\
& p_{T}^{\mu_{1} \ldots \mu_{n} \mu}\left(T_{s}\right)=0, \\
u_{\mu_{1}}\left(p_{s}\right) & \Rightarrow p_{T}^{\mu}\left(T_{s}\right)=P_{T}^{\mu}[\phi]=\epsilon_{A}^{\mu}\left(u\left(p_{s}\right)\right) P_{\phi}^{A} \approx p_{s}^{\mu}, \\
p_{T}^{\mu_{1} . \mu_{n} \mu}\left(T_{s}\right) u_{\mu}\left(p_{s}\right)= & \tilde{t}_{T}^{\mu_{1} \ldots \mu_{n}}\left(T_{s}\right)=\epsilon_{r_{1}}^{\mu_{1}}\left(u\left(p_{s}\right)\right) \ldots \epsilon_{r_{n}}^{\mu_{n}}\left(u\left(p_{s}\right)\right) I_{T}^{r_{1} . . r_{n} \tau \tau}\left(T_{s}\right) .
\end{aligned}
$$

The spin dipole is defined as

$$
\begin{aligned}
S_{T}^{\mu \nu}\left(T_{s}\right)[\phi]= & 2 p_{T}^{[\mu \nu]}\left(T_{s}\right)=2 \epsilon_{r}^{[\mu}\left(u\left(p_{s}\right)\right) \epsilon_{A}^{\nu]}\left(u\left(p_{s}\right)\right) I_{T}^{r A \tau}\left(T_{s}\right)= \\
= & {\left[\epsilon_{r}^{\mu}\left(u\left(p_{s}\right)\right) u^{\nu}\left(p_{s}\right)-\epsilon_{r}^{\nu}\left(u\left(p_{s}\right)\right) u^{\mu}\left(p_{s}\right)\right] \frac{1}{2} \int d^{3} \sigma \sigma^{r}\left[\pi^{2}+(\vec{\partial} \phi)^{2}+m^{2} \phi^{2}\right]\left(T_{s}, \vec{\sigma}\right)+} \\
+ & {\left[\epsilon_{r}^{\mu}\left(u\left(p_{s}\right)\right) \epsilon_{s}^{\nu}\left(u\left(p_{s}\right)\right)-\epsilon_{r}^{\nu}\left(u\left(p_{s}\right)\right) \epsilon_{s}^{\mu}\left(u\left(p_{s}\right)\right)\right] \int d^{3} \sigma \sigma^{r}\left[\pi \partial^{s} \phi\right]\left(T_{s}, \vec{\sigma}\right)=} \\
= & S_{s}^{\mu \nu}=\epsilon_{r}^{\mu}\left(u\left(p_{s}\right)\right) \epsilon_{s}^{\nu}\left(u\left(p_{s}\right)\right) S_{\phi}^{r s}+\left[\epsilon_{r}^{\mu}\left(u\left(p_{s}\right)\right) u^{\nu}\left(p_{s}\right)-\epsilon_{r}^{\nu}\left(u\left(p_{s}\right)\right) u^{\mu}\left(p_{s}\right)\right] S_{\phi}^{\tau r}= \\
= & \epsilon_{r}^{\mu}\left(u\left(p_{s}\right)\right) \epsilon_{s}^{\nu}\left(u\left(p_{s}\right)\right) \int d \tilde{q} \mathbf{H}\left(T_{s}, \vec{q}\right)\left(q^{r} \frac{\partial}{\partial q^{s}}-q^{s} \frac{\partial}{\partial q^{r}}\right) \mathbf{K}\left(T_{s}, \vec{q}\right)- \\
- & {\left[\epsilon_{r}^{\mu}\left(u\left(p_{s}\right)\right) u^{\nu}\left(p_{s}\right)-\epsilon_{r}^{\nu}\left(u\left(p_{s}\right)\right) u^{\mu}\left(p_{s}\right)\right] \int d \tilde{q} \omega(q) \mathbf{H}\left(T_{s}, \vec{q}\right) \frac{\partial}{\partial q^{r}} \mathbf{K}\left(T_{s}, \vec{q}\right), } \\
& u_{\mu}\left(p_{s}\right) S_{T}^{\mu \nu}\left(T_{s}\right)[\phi]=-\epsilon_{r}^{\nu}\left(u\left(p_{s}\right)\right) S_{\phi}^{\tau r}=-\tilde{t}_{T}^{\nu}\left(T_{s}\right)=P_{\phi}^{\tau} \epsilon_{r}^{\nu}\left(u\left(p_{s}\right)\right) r_{\phi}^{r},
\end{aligned}
$$


with $u_{\mu}\left(p_{s}\right) S_{T}^{\mu \nu}\left(T_{s}\right)[\phi]=0$ when $\tilde{t}_{T}^{\mu_{1}}\left(T_{s}\right)=0$ and this condition can be taken as a definition of center of mass equivalent to Dixon's one. When this condition holds, the barycentrice spin dipole is $S_{T}^{\mu \nu}\left(T_{s}\right)[\phi]=2 \epsilon_{r}^{[\mu}\left(u\left(p_{s}\right)\right) \epsilon_{s}^{\nu]}\left(u\left(p_{s}\right)\right) I_{T}^{r s \tau}\left(T_{s}\right)$, so that $I_{T}^{[r s] \tau}\left(T_{s}\right)=$ $\epsilon_{\mu}^{r}\left(u\left(p_{s}\right)\right) \epsilon_{\nu}^{s}\left(u\left(p_{s}\right)\right) S_{T}^{\mu \nu}\left(T_{s}\right)[\phi]$.

As shown in Ref. [9], if the Klein-Gordon field has a compact support $W$ on the Wigner hyperplanes $\Sigma_{W \tau}$ and if $f(x)$ is a $C^{\infty}$ complex-valued scalar function on Minkowski spacetime with compact support [so that its Fourier transform $\tilde{f}(k)=\int d^{4} x f(x) e^{i k \cdot x}$ is a slowly increasing entire analytic function on Minkowski spacetime $\left(\left|\left(x^{o}+i y^{o}\right)^{q_{o}} \ldots\left(x^{3}+i y^{3}\right)^{q_{3}} f\left(x^{\mu}+i y^{\mu}\right)\right|<\right.$ $C_{q_{o} \ldots q_{3}} e^{a_{o}\left|y^{o}\right|+\ldots+a_{3}\left|y^{3}\right|}, a_{\mu}>0, q_{\mu}$ positive integers for every $\mu$ and $\left.C_{q_{o} \ldots q_{3}}>0\right)$, whose inverse is $\left.f(x)=\int \frac{d^{4} k}{(2 \pi)^{4}} \tilde{f}(k) e^{-i k \cdot x}\right]$, we have

$$
\begin{aligned}
<T^{\mu \nu}, f>= & \int d^{4} x T^{\mu \nu}(x) f(x)= \\
= & \int d T_{s} \int d^{3} \sigma f\left(x_{s}+\delta x_{s}\right) T^{\mu \nu}\left[x_{s}\left(T_{s}\right)+\delta x_{s}(\vec{\sigma})\right][\phi]= \\
= & \int d T_{s} \int d^{3} \sigma \int \frac{d^{4} k}{(2 \pi)^{4}} \tilde{f}(k) e^{-i k \cdot\left[x_{s}\left(T_{s}\right)+\delta x_{s}(\vec{\sigma})\right]} T^{\mu \nu}\left[x_{s}\left(T_{s}\right)+\delta x_{s}(\vec{\sigma})\right][\phi]= \\
= & \int d T_{s} \int \frac{d^{4} k}{(2 \pi)^{4}} \tilde{f}(k) e^{-i k \cdot x_{s}\left(T_{s}\right)} \int d^{3} \sigma T^{\mu \nu}\left[x_{s}\left(T_{s}\right)+\delta x_{s}(\vec{\sigma})\right][\phi] \\
& \sum_{n=0}^{\infty} \frac{(-i)^{n}}{n !}\left[k_{\mu} \epsilon_{u}^{\mu}\left(u\left(p_{s}\right)\right) \sigma^{u}\right]^{n}= \\
= & \int d T_{s} \int \frac{d^{4} k}{(2 \pi)^{4}} \tilde{f}(k) e^{-i k \cdot x_{s}\left(T_{s}\right)} \sum_{n=0}^{\infty} \frac{(-i)^{n}}{n !} k_{\mu_{1} \ldots k_{\mu_{n}}} t_{T}^{\mu_{1} \ldots \mu_{n} \mu \nu}\left(T_{s}\right),
\end{aligned}
$$

and, but only for $f(x)$ analytic in W [9], we get

$$
\begin{aligned}
<T^{\mu \nu}, f>= & \left.\int d T_{s} \sum_{n=0}^{\infty} \frac{1}{n !} t_{T}^{\mu_{1} \ldots \mu_{n} \mu \nu}\left(T_{s}\right) \frac{\partial^{n} f(x)}{\partial x^{\mu_{1}} \ldots \partial x^{\mu_{n}}}\right|_{x=x_{s}\left(T_{s}\right)}, \\
& \Downarrow \\
T^{\mu \nu}(x)[\phi]= & \sum_{n=0}^{\infty} \frac{(-1)^{n}}{n !} \frac{\partial^{n}}{\partial x^{\mu_{1}} \ldots \partial x^{\mu_{n}}} \int d T_{s} \delta^{4}\left(x-x_{s}\left(T_{s}\right)\right) t_{T}^{\mu_{1} \ldots \mu_{n} \mu \nu}\left(T_{s}\right) .
\end{aligned}
$$

For a non analytic $f(x)$ we have

$$
\begin{aligned}
<T^{\mu \nu}, f> & =\left.\int d T_{s} \sum_{n=0}^{N} \frac{1}{n !} t_{T}^{\mu_{1} \ldots \mu_{n} \mu \nu}\left(T_{s}\right) \frac{\partial^{n} f(x)}{\partial x^{\mu_{1}} \ldots \partial x^{\mu_{n}}}\right|_{x=x_{s}\left(T_{s}\right)}+ \\
& +\int d T_{s} \int \frac{d^{4} k}{(2 \pi)^{4}} \tilde{f}(k) e^{-i k \cdot x_{s}\left(T_{s}\right)} \sum_{n=N+1}^{\infty} \frac{(-i)^{n}}{n !} k_{\mu_{1}} \ldots k_{\mu_{n}} t_{T}^{\mu_{1} \ldots \mu_{n} \mu \nu}\left(T_{s}\right),
\end{aligned}
$$

and, as shown in Ref. [9], from the knowledge of the moments $t_{T}^{\mu_{1} \ldots \mu_{n} \mu}\left(T_{s}\right)$ for all $n>N$ we can get $T^{\mu \nu}(x)$ and, thus, all the moments with $n \leq N$.

In Appendix C other types of Dixon's multipoles are analyzed. From this study it turns out that the multipolar expansion(5.15) may be rearranged with the help of the Hamilton equations implying $\partial_{\mu} T^{\mu \nu} \stackrel{\circ}{=} 0$, so that for analytic Klein-Gordon configurations from Eq. (C5) we get 


$$
\begin{aligned}
T^{\mu \nu}(x)[\phi] & \stackrel{\circ}{=} u^{(\mu}\left(p_{s}\right) \epsilon_{A}^{\nu)}\left(u\left(p_{s}\right)\right) \int d T_{s} \delta^{4}\left(x-x_{s}\left(T_{s}\right)\right) P_{\phi}^{A}+ \\
& +\frac{1}{2} \frac{\partial}{\partial x^{\rho}} \int d T_{s} \delta^{4}\left(x-x_{s}\left(T_{s}\right)\right) S_{T}^{\rho(\mu}\left(T_{s}\right)[\phi] u^{\nu)}\left(p_{s}\right)+ \\
& +\sum_{n=2}^{\infty} \frac{(-1)^{n}}{n !} \frac{\partial^{n}}{\partial x^{\mu_{1}} \ldots \partial x^{\mu_{n}}} \int d T_{s} \delta^{4}\left(x-x_{s}\left(T_{s}\right)\right) \mathcal{I}_{T}^{\mu_{1} . . \mu_{n} \mu \nu}\left(T_{s}\right)
\end{aligned}
$$

where for $n \geq 2 \quad \mathcal{I}_{T}^{\mu_{1} . . \mu_{n} \mu \nu}\left(T_{s}\right)=\frac{4(n-1)}{n+1} J_{T}^{\left(\mu_{1} . . \mu_{n-1}|\mu| \mu_{n}\right) \nu}\left(T_{s}\right)$, with the quantities $J_{T}^{\mu_{1} . \mu_{n} \mu \nu \rho \sigma}\left(T_{s}\right)$ being the Dixon $2^{2+n}$-pole inertial moment tensors given in Eqs.(C7) [the quadrupole and related inertia tensor are proportional to $\left.I_{T}^{r_{1} r_{2} \tau \tau}\left(T_{s}\right)\right]$.

The equations $\partial_{\mu} T^{\mu \nu} \stackrel{\circ}{=} 0$ imply the Papapetrou-Dixon-Souriau equations for the "poledipole' system $P_{T}^{\mu}\left(T_{s}\right)$ and $S_{T}^{\mu \nu}\left(T_{s}\right)[\phi]$ [see Eqs.(C1) and (C4)]

$$
\begin{aligned}
& \frac{d P_{T}^{\mu}\left(T_{s}\right)}{d T_{s}} \circ \\
&= 0 \\
& \frac{d S_{T}^{\mu \nu}\left(T_{s}\right)[\phi]}{d T_{s}} \stackrel{\circ}{=} 2 P_{T}^{[\mu}\left(T_{s}\right) u^{\nu]}\left(p_{s}\right)=2 P_{\phi}^{r} \epsilon_{r}^{[\mu}\left(u\left(p_{s}\right)\right) u^{\nu]}\left(p_{s}\right) \approx 0 .
\end{aligned}
$$




\section{EXTERNAL AND INTERNAL CANONICAL CENTER OF MASS, MOLLER'S CENTER OF ENERGY AND FOKKER-PRYCE CENTER OF INERTIA}

Let us now consider the problem of the definition of the relativistic center of mass of a Klein-Gordon field configuration, after having seen in the previous Section, Eq(5.12), Dixon's definition of this concept in the multipolar approach. Let us remark that in the approach leading to the rest-frame instant form of dynamics on Wigner's hyperplanes there is a splitting of this concept in an "external" and an "internal" one. One can either look at the isolated system from an arbitrary Lorentz frame or put himself inside the Wigner hyperplane.

From outside one finds after the canonical reduction to Wigner hyperplane that there is an origin $x_{s}^{\mu}(\tau)$ for these hyperplanes (a covariant noncanonical centroid) and a noncovariant canonical coordinate $\tilde{x}_{s}^{\mu}(\tau)$ describing an "external" decoupled point particle observer with a clock measuring the rest-frame time $T_{s}$. Associated with them there is the "external" realization (2.9) of the Poincaré group.

Instead, all the degrees of freedom of the isolated system (here the Klein-Gordon field configuration) are described by canonical variables on the Wigner hyperplane restricted by the rest-frame condition $\vec{P}_{\phi} \approx 0$, implying that an "internal" collective variable $\vec{X}_{\phi}$ is a gauge variable and that only relative variables are physical degrees of freedom (a form of weak Mach principle). Inside the Wigner hyperplane at $\tau=0$ there is another realization of the Poincaré group given by Eqs. (3.4), (3.5) with generators $P_{\phi}^{\tau}, P_{\phi}^{r}, J_{\phi}^{r s}, K_{\phi}^{r}=\left.J_{\phi}^{\tau r}\right|_{\tau=0}=$ $S_{\phi}^{\tau r}$, the "internal" Poincaré algebra. By using the methods of Ref. [10] (where there is a complete discussion of many definitions of relativistic center-of-mass-like variables) we can build the following three 'internal" (that is inside the Wigner hyperplane) Wigner 3-vectors corresponding to the 3 -vectors 'canonical center of mass' $\vec{q}_{\phi}$, 'Moller center of energy' $\vec{r}_{\phi}$ and 'Fokker-Pryce center of inertia' $\vec{y}_{\phi}$ (the analogous concepts for the relativistic N-body problem are under study [5]).

The noncanonical "internal" Møller center of energy and the associated spin 3-vector are

$$
\begin{aligned}
\vec{r}_{\phi}= & -\frac{\vec{K}_{\phi}}{P_{\phi}^{\tau}}=-\frac{1}{2 P_{\phi}^{\tau}} \int d^{3} \sigma \vec{\sigma}\left[\pi^{2}+(\vec{\partial} \phi)^{2}+m^{2} \phi^{2}\right](\tau, \vec{\sigma})= \\
= & -\frac{2}{P_{\phi}^{\tau}} \int d \tilde{q}_{1} d \tilde{q}_{2} \mathbf{A}_{\vec{q}_{1}}\left(\tau ; P_{\phi}^{A}, \mathbf{H}\right] \mathbf{A}_{\vec{q}_{2}}\left(\tau ; P_{\phi}^{A}, \mathbf{H}\right] \int d^{3} \sigma \vec{\sigma} \\
& \left(\left[\omega\left(q_{1}\right) \omega\left(q_{2}\right)+\vec{q}_{1} \cdot \vec{q}_{2}\right] \sin \left(\overrightarrow{q_{1}} \cdot \vec{\sigma}+\mathbf{B}_{\vec{q}_{1}}\left(\tau ; X_{\phi}^{A}, \mathbf{K}\right]\right) \sin \left(\overrightarrow{q_{2}} \cdot \vec{\sigma}+\mathbf{B}_{\vec{q}_{2}}\left(\tau ; X_{\phi}^{A}, \mathbf{K}\right]\right)+\right. \\
+ & \left.m^{2} \cos \left(\vec{q}_{1} \cdot \vec{\sigma}+\mathbf{B}_{\vec{q}_{1}}\left(\tau ; X_{\phi}^{A}, \mathbf{K}\right]\right) \cos \left(\vec{q}_{2} \cdot \vec{\sigma}+\mathbf{B}_{\vec{q}_{2}}\left(\tau ; X_{\phi}^{A}, \mathbf{K}\right]\right)\right), \\
\vec{\Omega}_{\phi}= & \vec{J}_{\phi}-\vec{r}_{\phi} \times \vec{P}_{\phi}, \\
& \left\{r_{\phi}^{r}, P_{\phi}^{s}\right\}=\delta^{r s}, \quad\left\{r_{\phi}^{r}, P_{\phi}^{\tau}\right\}=\frac{P_{\phi}^{r}}{P_{\phi}^{\tau}} \\
& \left\{r_{\phi}^{r}, r_{\phi}^{s}\right\}=-\frac{1}{\left(P_{\phi}^{\tau}\right)^{2}} \epsilon^{r s u} \Omega_{\phi}^{u},
\end{aligned}
$$




$$
\left\{\Omega_{\phi}^{r}, \Omega_{\phi}^{s}\right\}=\epsilon^{r s u}\left(\Omega_{\phi}^{u}-\frac{1}{\left(P_{\phi}^{\tau}\right)^{2}}\left(\vec{\Omega}_{\phi} \cdot \vec{P}_{\phi}\right) P_{\phi}^{u}\right), \quad\left\{\Omega_{\phi}^{r}, P_{\phi}^{\tau}\right\}=0 .
$$

We see that $\vec{r}_{\phi}$ coincides with Dixon's definition of Eq.(5.12).

The canonical "internal" center of mass $\left[\left\{q_{\phi}^{r}, q_{\phi}^{s}\right\}=0,\left\{q_{\phi}^{r}, P_{\phi}^{s}\right\}=\delta^{r s},\left\{J_{\phi}^{r}, q_{\phi}^{s}\right\}=\epsilon^{r s u} q_{\phi}^{u}\right]$ is

$$
\begin{aligned}
\vec{q}_{\phi}= & \vec{r}_{\phi}-\frac{\vec{J}_{\phi} \times \vec{\Omega}_{\phi}}{\sqrt{\left(P_{\phi}^{\tau}\right)^{2}-\vec{P}_{\phi}^{2}}\left(P_{\phi}^{\tau}+\sqrt{\left(P_{\phi}^{\tau}\right)^{2}-\vec{P}_{\phi}^{2}}\right)}= \\
= & -\frac{\vec{K}_{\phi}}{\sqrt{\left(P_{\phi}^{\tau}\right)^{2}-\vec{P}_{\phi}^{2}}}+\frac{\left(\vec{J}_{\phi} \times \vec{P}_{\phi}\right.}{\sqrt{\left(P_{\phi}^{\tau}\right)^{2}-\vec{P}_{\phi}^{2}}\left(P_{\phi}^{\tau}+\sqrt{\left(P_{\phi}^{\tau}\right)^{2}-\vec{P}_{\phi}^{2}}\right)}+ \\
+ & \frac{\left(\vec{P}_{\phi}\right) \vec{P}_{\phi}}{P_{\phi}^{\tau} \sqrt{\left(P_{\phi}^{\tau}\right)^{2}-\vec{P}_{\phi}^{2}}\left(P_{\phi}^{\tau}+\sqrt{\left(P_{\phi}^{\tau}\right)^{2}-\vec{P}_{\phi}^{2}}\right)}, \\
& \approx \vec{r}_{\phi} \quad \text { for } \quad \vec{P}_{\phi} \approx 0 ; \quad\left\{\vec{q}_{\phi}, P_{\phi}^{\tau}\right\}=\frac{\vec{P}_{\phi}}{P_{\phi}^{\tau}} \approx 0, \\
\vec{S}_{q \phi}= & \vec{J}_{\phi}-\vec{q}_{\phi} \times \vec{P}_{\phi}= \\
= & \frac{P_{\phi}^{\tau} \vec{J}_{\phi}}{\sqrt{\left(P_{\phi}^{\tau}\right)^{2}-\vec{P}_{\phi}^{2}}}+\frac{\vec{K}_{\phi} \times \vec{P}_{\phi}}{\sqrt{\left(P_{\phi}^{\tau}\right)^{2}-\vec{P}_{\phi}^{2}}}-\frac{\left(\vec{J}_{\phi} \cdot \vec{P}_{\phi}\right) \vec{P}_{\phi}}{\sqrt{\left(P_{\phi}^{\tau}\right)^{2}-\vec{P}_{\phi}^{2}}\left(P_{\phi}^{\tau}+\sqrt{\left(P_{\phi}^{\tau}\right)^{2}-\vec{P}_{\phi}^{2}}\right)} \approx \\
\approx & \vec{S}_{\phi}, \quad f o r \quad \vec{P}_{\phi} \approx 0, \quad\left\{\vec{S}_{q \phi}, \vec{P}_{\phi}\right\}=\left\{\vec{S}_{q \phi}, \vec{q}_{\phi}\right\}=0, \quad\left\{S_{q \phi}^{r}, S_{\phi \phi}^{s}\right\}=\epsilon^{r s u} S_{q \phi}^{u} .
\end{aligned}
$$

The "internal" noncanonical Fokker-Pryce center of inertia' $\vec{y}_{\phi}$ is

$$
\begin{gathered}
\vec{y}_{\phi}=\vec{q}_{\phi}+\frac{\vec{S}_{q \phi} \times \vec{P}_{\phi}}{\sqrt{\left(P_{\phi}^{\tau}\right)^{2}-\vec{P}_{\phi}^{2}}\left(P_{\phi}^{\tau}+\sqrt{\left(P_{\phi}^{\tau}\right)^{2}-\vec{P}_{\phi}^{2}}\right)}=\vec{r}_{\phi}+\frac{\vec{S}_{q \phi} \times \vec{P}_{\phi}}{P_{\phi}^{\tau} \sqrt{\left(P_{\phi}^{\tau}\right)^{2}-\vec{P}_{\phi}^{2}}}, \\
\vec{q}_{\phi}=\vec{r}_{\phi}+\frac{\vec{S}_{q \phi} \times \vec{P}_{\phi}}{P_{\phi}^{\tau}\left(P_{\phi}^{\tau}+\sqrt{\left(P_{\phi}^{\tau}\right)^{2}-\vec{P}_{\phi}^{2}}\right)}=\frac{P_{\phi}^{\tau} \vec{r}_{\phi}+\sqrt{\left(P_{\phi}^{\tau}\right)^{2}-\vec{P}_{\phi}^{2}} \vec{y}_{\phi}}{P_{\phi}^{\tau}+\sqrt{\left(P_{\phi}^{\tau}\right)^{2}-\vec{P}_{\phi}^{2}}}, \\
\left\{y_{\phi}^{r}, y_{\phi}^{s}\right\}=\frac{1}{P_{\phi}^{\tau} \sqrt{\left(P_{\phi}^{\tau}\right)^{2}-\vec{P}_{\phi}^{2}}} \epsilon^{r s u}\left[S_{q \phi}^{u}+\frac{\left(\vec{S}_{q \phi} \cdot \vec{P}_{\phi}\right) P_{\phi}^{u}}{\sqrt{\left(P_{\phi}^{\tau}\right)^{2}-\vec{P}_{\phi}^{2}}\left(P_{\phi}^{\tau}+\sqrt{\left(P_{\phi}^{\tau}\right)^{2}-\vec{P}_{\phi}^{2}}\right)},\right. \\
\vec{P}_{\phi} \approx 0 \Rightarrow \vec{q}_{\phi} \approx \vec{r}_{\phi} \approx \vec{y}_{\phi} .
\end{gathered}
$$

The Wigner 3-vector $\vec{q}_{\phi}$ is therefore the canonical 3-center of mass of the Klein-Gordon field [since $\vec{q}_{\phi} \approx \vec{r}_{\phi}$, it also describe that point $z^{\mu}\left(\tau, \vec{q}_{\phi}\right)=x_{s}^{\mu}(\tau)+q_{\phi}^{r} \epsilon_{r}^{\mu}\left(u\left(p_{s}\right)\right)$ where the energy of the field configuration is concentrated]. Instead, the previous variable $\vec{X}_{\phi}$ should be better named the 'center of phase' of the field configuration. There should exist a canonical transformation from the canonical basis $\tilde{X}_{\phi}^{\tau}, P_{\phi}^{\tau}, \vec{X}_{\phi}, \vec{P}_{\phi}, \mathbf{H}(\tau, \vec{k}), \mathbf{K}(\tau, \vec{k})$, to a new basis $q_{\phi}^{\tau}, P_{\phi}^{\tau^{\prime}}=\sqrt{\left(P_{\phi}^{\tau}\right)^{2}-\vec{P}_{\phi}^{2}}\left[\right.$ since $\left\{\vec{q}_{\phi}, P_{\phi}^{\tau}\right\}=\vec{P}_{\phi} / P_{\phi}^{\tau}$ only weakly zero], $\vec{q}_{\phi}, \vec{P}_{\phi}, \mathbf{H}^{\prime}(\tau, \vec{k}), \mathbf{K}^{\prime}(\tau, \vec{k})$ containing relative variables with respect to the true center of mass of the field configuration. 
However it does not seem easy to identify this final canonical basis; in particular it is not clear how to find the 'time' variable $q_{\phi}^{\tau}$. Maybe the methods used in Ref. [5] can be extended from particles to fields.

The gauge fixing $\vec{q}_{\phi} \approx 0$ [it also implies $\vec{\lambda}(\tau)=0$ like $\vec{X}_{\phi} \approx 0$ ] forces all three internal center-of-mass variables to coincide with the origin $x_{s}^{\mu}$ of the Wigner hyperplane. We shall denote $x_{s}^{\left(\vec{q}_{\phi}\right) \mu}(\tau)=x_{s}^{\mu}(0)+\tau u^{\mu}\left(p_{s}\right)$ the origin in this gauge, because, as we shall see, in this case it enjoys of properties not present in the $\vec{X}_{\phi} \approx 0$ gauge [where we have that $x_{s}^{\left(\vec{X}_{\phi}\right) \mu}(\tau)=$ $x_{s}^{\mu}(0)+\tau u_{s}^{\mu}\left(p_{s}\right)$ is formally equal to $x_{s}^{\left(\vec{q}_{\phi}\right) \mu}(\tau)$, but, since $\tilde{t}_{T}^{\mu}\left(T_{s}\right) \neq 0, u_{\mu}\left(p_{s}\right) S_{T}^{\mu \nu}\left(T_{s}\right)[\phi] \neq 0$, it has to be interpreted as a different centroid].

In the gauge $\overrightarrow{q_{\phi}} \approx 0$ the origin becomes the Dixon's center of mass which coincide with the internal Moller center of energy as shown in Eq.(5.12) [and then Eq.(5.16) implies that the origin is also the Tulczyjew [16] and the Pirani [17] centroid (see Ref. [18] for a review of these concepts in relation with the Papapetrou-Dixon-Souriau pole-dipole approximation of an extended object), because they are defined by $p_{s \mu} S_{T}^{\mu \nu}\left(T_{s}\right)[\phi] \approx 0$ and by $u_{\mu}\left(p_{s}\right) S_{T}^{\mu \nu}\left(T_{s}\right)[\phi] \approx 0$ respectively and we have $\left.p_{s}^{\mu}=\epsilon_{s} u^{\mu}\left(p_{s}\right)\right]$. Therefore, the worldline $x_{s}^{\left(\vec{q}_{\phi}\right) \mu}$ is the unique centerof-mass worldline of special relativity in the sense of Refs. [19].

The rest-frame instant form "external" realization of the Poincaré algebra of Eq. (2.9) has the generators $p_{s}^{\mu}, J_{s}^{i j}=\tilde{x}_{s}^{i} p_{s}^{j}-\tilde{x}_{s}^{j} p_{s}^{i}+\delta^{i r} \delta^{j s} S_{\phi}^{r s}, K_{s}^{i}=J_{s}^{o i}=\tilde{x}_{s}^{o} p_{s}^{i}-\tilde{x}_{s}^{i} p_{s}^{o}-\frac{\delta^{i r} S_{\phi}^{r s} p_{s}^{s}}{p_{s}^{o}+\epsilon_{s}}=$ $\tilde{x}_{s}^{o} p_{s}^{i}-\tilde{x}_{s}^{i} p_{s}^{o}+\delta^{i r} \frac{\left(\vec{S}_{\phi} \times \vec{p}_{s}\right)^{r}}{p_{s}^{o}+\epsilon_{s}}$ [for $\tilde{x}_{s}^{o}=0$ this is the Newton-Wigner decomposition of $\left.J_{s}^{\mu \nu}\right]$. As already said the canonical variables $\tilde{x}_{s}^{\mu}, p_{s}^{\mu}$, may be replaced by the canonical pairs $\epsilon_{s}= \pm \sqrt{p_{s}^{2}}, T_{s}=p_{s} \cdot \tilde{x}_{s} / \epsilon_{s}$ [to be gauge fixed with $T_{s}-\tau \approx 0$ ]; $\vec{k}_{s}=\vec{p}_{s} / \epsilon_{s}=\vec{u}\left(p_{s}\right)$, $\vec{z}_{s}=\epsilon_{s}\left(\overrightarrow{\widetilde{x}}_{s}-\frac{\vec{p}_{s}}{p_{s}^{o}} \tilde{x}_{s}^{o}\right) \equiv \epsilon_{s} \vec{q}_{s}$.

One can build three "external" 3 -variables, the canonical $\vec{Q}_{s}$, the Moller $\vec{R}_{s}$ and the Fokker-Pryce $\vec{Y}_{s}$ by using this rest-frame "external" realization of the Poincaré algebra

$$
\begin{aligned}
\vec{R}_{s}= & -\frac{1}{p_{s}^{o}} \vec{K}_{s}=\left(\overrightarrow{\tilde{x}}_{s}-\frac{\vec{p}_{s}}{p_{s}^{o}} \tilde{x}_{s}^{o}\right)-\frac{\vec{S}_{\phi} \times \vec{p}_{s}}{p_{s}^{o}\left(p_{s}^{o}+\epsilon_{s}\right)} \\
\vec{Q}_{s}= & \overrightarrow{\tilde{x}}_{s}-\frac{\vec{p}_{s}}{p_{s}^{o}} \tilde{x}_{s}^{o}=\frac{\vec{z}_{s}}{\epsilon_{s}}=\vec{R}_{s}+\frac{\vec{S}_{\phi} \times \vec{p}_{s}}{p_{s}^{o}\left(p_{s}^{o}+\epsilon_{s}\right)}=\frac{p_{s}^{o} \vec{R}_{s}+\epsilon_{s} \vec{Y}_{s}}{p_{s}^{o}+\epsilon_{s}} \\
\vec{Y}_{s}= & \vec{Q}_{s}+\frac{\vec{S}_{\phi} \times \vec{p}_{s}}{\epsilon_{s}\left(p_{s}^{o}+\epsilon_{s}\right)}=\vec{R}_{s}+\frac{\vec{S}_{\phi} \times \vec{p}_{s}}{p_{s}^{o} \epsilon_{s}}, \\
& \left\{R_{s}^{r}, R_{s}^{s}\right\}=-\frac{1}{\left(p_{s}^{o}\right)^{2}} \epsilon^{r s u} \Omega_{s}^{u}, \quad \vec{\Omega}_{s}=\vec{J}_{s}-\vec{R}_{s} \times \vec{p}_{s}, \\
& \left\{Y_{s}^{r}, Y_{s}^{s}\right\}=\frac{1}{\epsilon_{s} p_{s}^{o}} \epsilon^{r s u}\left[S_{\phi}^{u}+\frac{\left(\vec{S}_{\phi} \cdot \vec{p}_{s}\right) p_{s}^{u}}{\epsilon_{s}\left(p_{s}^{o}+\epsilon_{s}\right)}\right] \\
\vec{p}_{s}=0 \Rightarrow & \vec{Q}_{s}=\vec{Q}_{s}=\vec{p}_{s} \cdot \vec{R}_{s}=\vec{R}_{s},
\end{aligned}
$$

with the same velocity and coinciding in the Lorentz rest frame where $\stackrel{\circ}{p}_{s}^{\mu}=\epsilon_{s}(1 ; \overrightarrow{0})$

In Ref. [10 in a one-time framework without constraints and at a fixed time, it is shown that the 3-vector $\vec{Y}_{s}$ [but not $\vec{Q}_{s}$ and $\vec{R}_{s}$ ] satisfies the condition $\left\{K_{s}^{r}, Y_{s}^{s}\right\}=Y_{s}^{r}\left\{Y_{s}^{s}, p_{s}^{o}\right\}$ for 
being the space component of a 4-vector $Y_{s}^{\mu}$. In the enlarged canonical treatment including time variables, it is not clear which are the time components to be added to $\vec{Q}_{s}, \vec{R}_{s}, \vec{Y}_{s}$, to rebuild 4-dimesnional quantities $\tilde{x}_{s}^{\mu}, R_{s}^{\mu}, Y_{s}^{\mu}$, in an arbitrary Lorentz frame $\Gamma$, in which the origin of the Wigner hyperplane is the 4 -vector $x_{s}^{\mu}=\left(x_{s}^{o} ; \vec{x}_{s}\right)$. We have

$$
\begin{aligned}
\tilde{x}_{s}^{\mu}(\tau) & =\left(\tilde{x}_{s}^{o}(\tau) ; \overrightarrow{\tilde{x}}_{s}(\tau)\right)=x_{s}^{\mu}-\frac{1}{\epsilon_{s}\left(p_{s}^{o}+\epsilon_{s}\right)}\left[p_{s \nu} S_{s}^{\nu \mu}+\epsilon_{s}\left(S_{s}^{o \mu}-S_{s}^{o \nu} \frac{p_{s \nu} p_{s}^{\mu}}{\epsilon_{s}^{2}}\right)\right], \quad p_{s}^{\mu}, \\
\tilde{x}_{s}^{o} & =\sqrt{1+\vec{k}_{s}^{2}}\left(T_{s}+\frac{\vec{k}_{s} \cdot \vec{z}_{s}}{\epsilon_{s}}\right)=\sqrt{1+\vec{k}_{s}^{2}}\left(T_{s}+\vec{k}_{s} \cdot \vec{q}_{s}\right) \neq x_{s}^{0}, \quad p_{s}^{o}=\epsilon_{s} \sqrt{1+\vec{k}_{s}^{2}} \\
\overrightarrow{\tilde{x}}_{s} & =\frac{\vec{z}_{s}}{\epsilon_{s}}+\left(T_{s}+\frac{\vec{k}_{s} \cdot \vec{z}_{s}}{\epsilon_{s}}\right) \vec{k}_{s}=\vec{q}_{s}+\left(T_{s}+\vec{k}_{s} \cdot \vec{q}_{s}\right) \vec{k}_{s}, \quad \vec{p}_{s}=\epsilon_{s} \vec{k}_{s} .
\end{aligned}
$$

for the non-covariant (frame-dependent) canonical center of mass and its conjugate momentum.

Each Wigner hyperplane intersects the worldline of the arbitrary origin 4-vector $x_{s}^{\mu}(\tau)=$ $z^{\mu}(\tau, \overrightarrow{0})$ in $\vec{\sigma}=0$, the pseudo worldline of $\tilde{x}_{s}^{\mu}(\tau)=z^{\mu}(\tau, \tilde{\vec{\sigma}})$ in some $\tilde{\vec{\sigma}}$ and the worldline of the Fokker-Pryce 4-vector $Y_{s}^{\mu}(\tau)=z^{\mu}\left(\tau, \vec{\sigma}_{Y}\right)$ in some $\vec{\sigma}_{Y}$ [on this worldline one can put the "internal center of mass" with the gauge fixing $\vec{q}_{\phi} \approx 0\left(\vec{q}_{\phi} \approx \vec{r}_{\phi} \approx \vec{y}_{\phi}\right.$ due to $\left.\left.\vec{P}_{\phi} \approx 0\right)\right]$; one also has $R_{s}^{\mu}=z^{\mu}\left(\tau, \vec{\sigma}_{R}\right)$. Since we have $T_{s}=u\left(p_{s}\right) \cdot x_{s}=u\left(p_{s}\right) \cdot \tilde{x}_{s} \equiv \tau$ on the Wigner hyperplane labelled by $\tau$, we require that also $Y_{s}^{\mu}, R_{s}^{\mu}$ have time components such that they too satisfy $u\left(p_{s}\right) \cdot Y_{s}=u\left(p_{s}\right) \cdot R_{s}=T_{s} \equiv \tau$. Therefore, it is reasonable to assume that $\tilde{x}_{s}^{\mu}$, $Y_{s}^{\mu}$ and $R_{s}^{\mu}$ satisfy the following equations consistently with Eqs. (6.1), (6.2) when $T_{s} \equiv \tau$ and $\vec{q}_{\phi} \approx 0$

$$
\begin{aligned}
\tilde{x}_{s}^{\mu} & =\left(\tilde{x}_{s}^{o} ; \overrightarrow{\tilde{x}}_{s}\right)=\left(\tilde{x}_{s}^{o} ; \frac{\vec{z}_{s}}{\epsilon_{s}}+\left(T_{s}+\frac{\vec{k}_{s} \cdot \vec{z}_{s}}{\epsilon_{s}}\right) \vec{k}_{s}\right)=x_{s}^{\left(\vec{q}_{\phi}\right) \mu}+\epsilon_{u}^{\mu}\left(u\left(p_{s}\right)\right) \tilde{\sigma}^{u} \\
Y_{s}^{\mu} & =\left(\tilde{x}_{s}^{o} ; \frac{1}{\epsilon_{s}}\left[\vec{z}_{s}+\frac{\vec{S}_{\phi} \times \vec{p}_{s}}{\epsilon_{s}\left[1+u^{o}\left(p_{s}\right)\right]}\right]+\left(T_{s}+\frac{\vec{z}_{s}}{\epsilon_{s}}\right) \vec{k}_{s}\right)= \\
& =\tilde{x}_{s}^{\mu}+\eta_{r}^{\mu} \frac{\left(\vec{S}_{\phi} \times \vec{p}_{s}\right)^{r}}{\epsilon_{s}\left[1+u^{o}\left(p_{s}\right)\right]}= \\
& =x_{s}^{\left(\vec{q}_{\phi}\right) \mu}+\epsilon_{u}^{\mu}\left(u\left(p_{s}\right)\right) \sigma_{Y}^{u}, \\
R_{s}^{\mu} & =\left(\tilde{x}_{s}^{o} ; \frac{1}{\epsilon_{s}}\left[\vec{z}_{s}-\frac{\vec{S}_{\phi} \times \vec{p}_{s}}{\epsilon_{s} u^{o}\left(p_{s}\right)\left[1+u^{o}\left(p_{s}\right)\right]}\right]+\left(T_{s}+\frac{\vec{k}_{s} \cdot \vec{z}_{s}}{\epsilon_{s}}\right) \vec{k}_{s}\right)= \\
& =\tilde{x}_{s}^{\mu}-\eta_{r}^{\mu} \frac{\left(\vec{S}_{\phi} \times \vec{p}_{s}\right)^{r}}{\epsilon_{s} u^{o}\left(p_{s}\right)\left[1+u^{o}\left(p_{s}\right)\right]}= \\
& =x_{s}^{\left(\vec{q}_{\phi}\right) \mu}+\epsilon_{u}^{\mu}\left(u\left(p_{s}\right)\right) \sigma_{R}^{u}, \\
T_{s} & =u\left(p_{s}\right) \cdot x_{s}^{\left(\vec{q}_{\phi}\right)}=u\left(p_{s}\right) \cdot \tilde{x}_{s}=u\left(p_{s}\right) \cdot Y_{s}=u\left(p_{s}\right) \cdot R_{s}, \\
\tilde{\sigma}^{r} & =\epsilon_{r \mu}\left(u\left(p_{s}\right)\right)\left[x_{s}^{\left(\vec{q}_{\phi}\right) \mu}-\tilde{x}_{s}^{\mu}\right]=\frac{\epsilon_{r \mu}\left(u\left(p_{s}\right)\right)\left[u_{\nu}\left(p_{s}\right) S_{s}^{\nu \mu}+S_{s}^{o \mu}\right]}{\left[1+u^{o}\left(p_{s}\right)\right]} \\
& =-S_{\phi}^{\tau r}+\frac{S_{\phi}^{r s} p_{s}^{s}}{\epsilon_{s}\left[1+u^{o}\left(p_{s}\right)\right]}=\epsilon_{s} r_{\phi}^{r}+\frac{S_{\phi}^{r s} u^{s}\left(p_{s}\right)}{1+u^{o}\left(p_{s}\right)} \approx
\end{aligned}
$$




$$
\begin{aligned}
& \approx \epsilon_{s} q_{\phi}^{r}+\frac{S_{\phi}^{r s} u^{s}\left(p_{s}\right)}{1+u^{o}\left(p_{s}\right)} \approx \frac{S_{\phi}^{r s} u^{s}\left(p_{s}\right)}{1+u^{o}\left(p_{s}\right)} \\
\sigma_{Y}^{r} & =\epsilon_{r \mu}\left(u\left(p_{s}\right)\right)\left[x_{s}^{\left(\vec{q}_{\phi}\right) \mu}-Y_{s}^{\mu}\right]=\tilde{\sigma}^{r}-\epsilon_{r u}\left(u\left(p_{s}\right)\right) \frac{\left(\vec{S}_{\phi} \times \vec{p}_{s}\right)^{u}}{\epsilon_{s}\left[1+u^{o}\left(p_{s}\right)\right]}= \\
& =\tilde{\sigma}^{r}+\frac{S_{\phi}^{r s} u^{s}\left(p_{s}\right)}{1+u^{o}\left(p_{s}\right)}=\epsilon_{s} r_{\phi}^{r} \approx \epsilon_{s} q_{\phi}^{r} \approx 0, \\
\sigma_{R}^{r} & =\epsilon_{r \mu}\left(u\left(p_{s}\right)\right)\left[x_{s}^{\left(\vec{q}_{\phi}\right) \mu}-R_{s}^{\mu}\right]=\tilde{\sigma}^{r}+\epsilon_{r u}\left(u\left(p_{s}\right)\right) \frac{\left(\vec{S}_{\phi} \times \vec{p}_{s}\right)^{u}}{\epsilon_{s} u^{o}\left(p_{s}\right)\left[1+u^{o}\left(p_{s}\right)\right]}= \\
& =\tilde{\sigma}^{r}-\frac{S_{\phi}^{r s} u^{s}\left(p_{s}\right)}{u^{o}\left(p_{s}\right)\left[1+u^{o}\left(p_{s}\right)\right]}=\epsilon_{s} r_{\phi}^{r}+\frac{\left[1-u^{o}\left(p_{s}\right)\right] S_{\phi}^{r s} u^{s}\left(p_{s}\right)}{u^{o}\left(p_{s}\right)\left[1+u^{o}\left(p_{s}\right)\right]} \approx \\
& \approx \frac{\left[1-u^{o}\left(p_{s}\right)\right] S_{\phi}^{r s} u^{s}\left(p_{s}\right)}{u^{o}\left(p_{s}\right)\left[1+u^{o}\left(p_{s}\right)\right]}, \\
& \Rightarrow x_{s}^{\left(\vec{q}_{\phi}\right) \mu}(\tau)=Y_{s}^{\mu}, \quad \text { for } \quad \vec{q}_{\phi} \approx 0,
\end{aligned}
$$

namely in the gauge $\vec{q}_{\phi} \approx 0$ the external Fokker-Pryce non canonical center of inertia coincides with the origin $x_{s}^{\left(\vec{q}_{\phi}\right) \mu}(\tau)$ carrying the "internal" center of mass (coinciding with the "internal" Möller center of energy and with the "internal" Fokker-Pryce center of inertia) and also being the Pirani centroid and the Tulczyjew centroid.

Therefore, if we would find the canonical basis $q_{\phi}^{\tau}, P_{\phi}^{\tau^{\prime}}=\sqrt{\left(P_{\phi}^{\tau}\right)^{2}-\vec{P}_{\phi}^{2}}, \vec{q}_{\phi}, \vec{P}_{\phi}, \mathbf{H}^{\prime}(\tau, \vec{q})$, $\mathbf{K}^{\prime}(\tau, \vec{q})$, then, in the gauge $\vec{q}_{\phi} \approx 0$ and $T_{s} \approx \tau$, the Klein-Gordon field configurations would have the four-momentum density peaked on the worldline $x_{s}^{\left(\vec{q}_{\phi}\right) \mu}\left(T_{s}\right)$; the canonical variables $\mathbf{H}^{\prime}(\tau, \vec{q}), \mathbf{K}^{\prime}(\tau, \vec{q})$ would characterize the relative motions with respect to the "monopole" configuration describing the center of mass of the field configuration. The "monopole" solutions of the Klein-Gordon equation would be identified by the conditions $\mathbf{H}^{\prime}(\tau, \vec{q})=$ $\mathbf{K}^{\prime}(\tau, \vec{q})=0$ [formally they are given by Eqs.(4.38) with $X_{\phi}^{\tau}, \vec{X}_{\phi}$ and $P_{\phi}^{\tau}$ replaced by $q_{\phi}^{\tau}, \vec{q}_{\phi}$ and $\sqrt{\left(P_{\phi}^{\tau}\right)^{2}-\vec{P}_{\phi}^{2}}$ ]: these field configurations have the same independent degrees of freedom of a free scalar particle at rest with mass $P_{\phi}^{\tau^{\prime}} \approx P_{\phi}^{\tau}$ [its conjugate "time" $q_{\phi}^{\tau}$ would satisfy $\partial / \partial T_{s} \stackrel{\circ}{=} \partial \partial q_{\phi}^{\tau}$ in the free case, see Subsection D of Section IV].

Remember that the canonical center of mass lies in between the Moller center of energy and the Fokker-Pryce center of inertia and that the noncovariance region around the FokkerPryce 4-vector extends to a worldtube with radius (the Moller radius) $\left|\vec{S}_{\phi}\right| / P_{\phi}^{\tau}$. 


\section{COUPLING TO SCALAR PARTICLES.}

In this Section we shall consider a Yukawa-type coupling of the Klein-Gordon field to the masses of scalar relativistic particles [1] to find which are the Dirac observables spanning the reduced phase space. The action is

$$
\begin{aligned}
S= & \int d \tau L(\tau)=\int d \tau d^{3} \sigma(N(\tau, \vec{\sigma}) \sqrt{\gamma(\tau, \vec{\sigma})} \\
& \frac{1}{2}\left[g^{\tau \tau} \dot{\phi}^{2}+2 g^{\tau \check{r}} \dot{\phi} \partial_{\check{r}} \phi+g^{\check{r} \check{s}} \partial_{\breve{r}} \phi \partial_{\breve{s}} \phi-m^{2} \phi^{2}\right](\tau, \vec{\sigma})- \\
- & \sum_{i=1}^{N} \delta^{3}\left(\vec{\sigma}-\vec{\eta}_{i}(\tau)\right)\left[\eta_{i} m_{i}+G \phi(\tau, \vec{\sigma})\right] \sqrt{\left.\left[g_{\tau \tau}+2 g_{\tau \check{r}} \ddot{\eta}_{i}^{\check{r}}(\tau)+g_{\check{r} \check{s}} \dot{\eta}_{i}^{\check{r}}(\tau) \dot{\eta}_{i}^{\check{s}}(\tau)\right](\tau, \vec{\sigma})\right)}= \\
= & \int d \tau d^{3} \sigma\left(\sqrt { \gamma ( \tau , \vec { \sigma } ) } \frac { 1 } { 2 } \left[\frac{1}{N}\left[\partial_{\tau}-N^{\check{r}} \partial_{\check{r}}\right] \phi\left[\partial_{\tau}-N^{\check{s}} \partial_{\check{s}}\right] \phi+\right.\right. \\
+ & \left.N\left[\gamma^{\check{r} \check{s}} \partial_{\check{r}} \phi \partial_{\check{s}} \phi-m^{2} \phi^{2}\right]\right](\tau, \vec{\sigma})-\sum_{i=1}^{N} \delta^{3}\left(\vec{\sigma}-\vec{\eta}_{i}(\tau)\right)\left[\eta_{i} m_{i}+G \phi(\tau, \vec{\sigma})\right] \\
& \left.\sqrt{\left[N^{2}+g_{\check{r} \check{s}}\left(N^{\check{r}}+\dot{\eta}_{i}^{\check{r}}(\tau)\right)\left(N^{\check{s}}+\dot{\eta}_{i}^{\check{s}}(\tau)\right)\right](\tau, \vec{\sigma})}\right)
\end{aligned}
$$

and the canonical momenta are

$$
\begin{aligned}
& \kappa_{i \check{r}}(\tau)=-\frac{\partial L(\tau)}{\partial \dot{\eta}_{i}^{r}}=\left[\eta_{i} m_{i}+G \phi\left(\tau, \vec{\eta}_{i}(\tau)\right)\right] \\
& \frac{g_{\check{r} \check{s}}\left(N^{\check{s}}+\dot{\eta}_{i}^{\check{s}}(\tau)\right)}{\sqrt{N^{2}+g_{\check{r} \check{s}}\left(N^{\check{r}}+\dot{\eta}_{i}^{\check{r}}(\tau)\right)\left(N^{\check{s}}+\dot{\eta}_{i}^{\check{s}}(\tau)\right)}}\left(\tau, \vec{\eta}_{i}(\tau)\right), \\
& \pi(\tau, \vec{\sigma})=\frac{\partial L}{\partial \partial_{\tau} \phi(\tau, \vec{\sigma})}= \\
& =\frac{\sqrt{\gamma}(\tau, \vec{\sigma})}{N(\tau, \vec{\sigma})}\left[\dot{\phi}-N^{\check{r}} \partial_{\check{r}} \phi\right](\tau, \vec{\sigma}), \\
& \rho_{\mu}(\tau, \vec{\sigma})=-\frac{\partial L}{\partial \partial_{\tau} z^{\mu}(\tau, \vec{\sigma})}= \\
& =l_{\mu}(\tau, \vec{\sigma})\left[\left(\frac{\sqrt{\gamma}}{2}\left[\frac{1}{N^{2}}\left(\dot{\phi}-N^{\check{r}} \partial_{\check{r}} \phi\right)^{2}-\gamma^{\check{r} \check{s}} \partial_{\check{r}} \phi \partial_{\breve{s}} \phi+m^{2} \phi^{2}\right]\right)(\tau, \vec{\sigma})+\right. \\
& \left.+\sum_{i=1}^{N} \delta^{3}\left(\vec{\sigma}-\vec{\eta}_{i}(\tau)\right) \frac{\left[\eta_{i} m_{i}+G \phi(\tau, \vec{\sigma})\right] N(\tau, \vec{\sigma})}{\sqrt{\left[N^{2}+g_{\check{r} \check{s}}\left(N^{\check{r}}+\dot{\eta}_{i}^{\check{r}}(\tau)\right)\left(N^{\check{s}}+\dot{\eta}_{i}^{\breve{s}}(\tau)\right)\right](\tau, \vec{\sigma})}}\right]+ \\
& +z_{\check{s} \mu}(\tau, \vec{\sigma}) \gamma^{\breve{s} \check{r}}(\tau, \vec{\sigma})\left[\left(\frac{\sqrt{\gamma}}{N} \partial_{\breve{r}} \phi\left(\dot{\phi}-N^{\check{u}} \partial_{\breve{u}} \phi\right)\right)(\tau, \vec{\sigma})+\right. \\
& \left.+\sum_{i=1}^{N} \delta^{3}\left(\vec{\sigma}-\vec{\eta}_{i}(\tau)\right) \frac{\left[\eta_{i} m_{i}+G \phi\right] g_{\check{r} \check{s}}\left(N^{\check{u}}+\dot{\eta}_{i}^{\check{u}}(\tau)\right)}{\sqrt{N^{2}+g_{\check{r} \check{s}}\left(N^{\check{r}}+\dot{\eta}_{i}^{\check{r}}(\tau)\right)\left(N^{\check{s}}+\dot{\eta}_{i}^{\breve{s}}(\tau)\right)}}(\tau, \vec{\sigma})\right] .
\end{aligned}
$$

We get the first class constraints

$$
\mathcal{H}_{\mu}(\tau, \vec{\sigma})=\rho_{\mu}(\tau, \vec{\sigma})-
$$




$$
\begin{aligned}
& -l_{\mu}(\tau, \vec{\sigma})\left[\left(\frac{\pi^{2}}{2 \sqrt{\gamma}}-\frac{\sqrt{\gamma}}{2}\left[\gamma^{\check{r} \check{s}} \partial_{\check{r}} \phi \partial_{\check{s}} \phi-m^{2} \phi^{2}\right]\right)(\tau, \vec{\sigma})+\right. \\
& +\sum_{i=1}^{N} \delta^{3}\left(\vec{\sigma}-\vec{\eta}_{i}(\tau)\right) \eta_{i} \sqrt{\left.\left[\eta_{i} m_{i}+G \phi(\tau, \vec{\sigma})\right]^{2}-\gamma^{\check{r} \check{s}}(\tau, \vec{\sigma}) \kappa_{i \check{r}}(\tau) \kappa_{i \check{s}}(\tau)\right]}- \\
& -z_{\breve{s} \mu}(\tau, \vec{\sigma}) \gamma^{\check{r} \check{s}}(\tau, \vec{\sigma})\left[\left(\pi \partial_{\check{r}} \phi\right)(\tau, \vec{\sigma})+\sum_{i=1}^{N} \delta^{3}\left(\vec{\sigma}-\vec{\eta}_{i}(\tau)\right) \kappa_{i \check{r}}(\tau)\right] \approx 0 .
\end{aligned}
$$

Following the procedure of Section II, one can arrive at the reduction on the Wigner hyperplanes, where the remaining four first class constraints and Dirac Hamiltonian are

$$
\begin{aligned}
\mathcal{H}(\tau) & =\epsilon_{s}-\left[\sum_{i=1}^{N} \eta_{i} \sqrt{\left[\eta_{i} m_{i}+G \phi\left(\tau, \vec{\eta}_{i}(\tau)\right)\right]^{2}+\vec{\kappa}_{i}^{2}(\tau)}+\right. \\
& \left.+\frac{1}{2} \int d^{3} \sigma\left[\pi^{2}+(\vec{\partial} \phi)^{2}+m^{2} \phi^{2}\right](\tau, \vec{\sigma})\right]=\epsilon_{s}-M \approx 0 \\
\overrightarrow{\mathcal{H}}_{p}(\tau) & =\sum_{i=1}^{N} \vec{\kappa}_{i}(\tau)+\int d^{3} \sigma[\pi \vec{\partial} \phi](\tau, \vec{\sigma}) \approx 0 \\
H_{D} & =\lambda(\tau) \mathcal{H}+\vec{\lambda}(\tau) \cdot \overrightarrow{\mathcal{H}}_{p} .
\end{aligned}
$$

In the gauge $T_{s}-\tau \approx 0$ the Hamiltonian is $H_{R}=M-\vec{\lambda}(\tau) \cdot \overrightarrow{\mathcal{H}}_{p}$.

Let us make a canonical transformation from the canonical basis $\vec{\eta}_{i}(\tau), \vec{\kappa}_{i}(\tau), \phi(\tau, \vec{\sigma})$, $\pi(\tau, \vec{\sigma})$, to the new basis $\vec{\eta}_{+}(\tau), \vec{\kappa}_{+}(\tau), \vec{\rho}_{a}(\tau), \vec{\pi}_{a}(\tau), X_{\phi}^{\tau}, P_{\phi}^{\tau}, \vec{X}_{\phi}, \vec{P}_{\phi}, \mathbf{H}(\tau, \vec{q}), \mathbf{K}(\tau, \vec{q})[\phi, \pi$, are assumed to satisfy Eq.(4.29)] with the particle variables defined by

$$
\begin{aligned}
\vec{\eta}_{i}= & \vec{\eta}_{+}+\frac{1}{\sqrt{N}} \sum_{a=1}^{N-1} \hat{\gamma}_{a i} \vec{\rho}_{a}, \\
\vec{\kappa}_{i}= & \frac{1}{N} \vec{\kappa}_{+}+\sqrt{N} \sum_{a=1}^{N-1} \hat{\gamma}_{a i} \vec{\pi}_{a}, \\
\vec{\eta}_{+}= & \frac{1}{N} \sum_{i=1}^{N} \vec{\eta}_{i}, \quad \vec{\rho}_{a}=\sqrt{N} \sum_{i=1}^{N} \hat{\gamma} \vec{\eta}_{i}, \\
\vec{\kappa}_{+}= & \sum_{i=1}^{N} \vec{\kappa}_{i}, \quad \vec{\pi}_{a}=\frac{1}{\sqrt{N}} \sum_{i=1}^{N} \hat{\gamma}_{a i} \vec{\kappa}_{i}, \\
& \sum_{i=1}^{N} \hat{\gamma}_{a i}=0, \quad \sum_{a=1}^{N-1} \hat{\gamma}_{a i} \hat{\gamma}_{a j}=\delta_{i j}-\frac{1}{N}, \quad \sum_{i=1}^{N} \hat{\gamma}_{a i} \hat{\gamma}_{b i}=\delta_{a b} .
\end{aligned}
$$

The variable $\vec{\eta}_{+}(\tau)$ is playing the role of a naive "internal" center of mass for the particles on the Wigner hyperplane. From the discussion of the previous Section, it is clear that the real "internal" center of mass of the $\mathrm{N}$ particles is a $\vec{q}_{+}$defined like $\vec{q}_{\phi}$ of Eq.(6.2) with $\vec{r}_{+}=\sum_{i=1}^{N} \sqrt{m_{i}^{2}+\vec{\kappa}_{i}^{2}} \vec{\eta}_{i} / \sum_{k=1}^{N} \sqrt{m_{k}^{2}+\vec{\kappa}_{k}^{2}}$. But, since it is not known (like it happens for the Klein-Gordon field) the canonical transformation $\vec{\eta}_{i}, \vec{\kappa}_{i} \mapsto \vec{q}_{+}, \vec{\kappa}_{+}, \vec{\rho}_{q a}, \vec{\pi}_{q a}$ identifying the real relative variables $\vec{\rho}_{q a}, \vec{\pi}_{q a}$ [see however Ref. [5]], we shall go on with the previous naive canonical transformation in the following discussion to see which kind of collective and relative variables emerge for the particles plus the real Klein-Gordon field. 
We also remark that we use the field "center of phase" $\vec{X}_{\phi}$ and not the real internal center of mass $\vec{q}_{\phi}$, because the knowledge of the canonical basis containing $\vec{X}_{\phi}$ allows us to illustrate interpretational aspects which will hold also with the canonical basis containing $\vec{q}_{\phi}$ when it will be found.

The constraints become

$$
\begin{aligned}
\mathcal{H}(\tau) & =\epsilon_{s}-\left[P_{\phi}^{\tau}+\sum_{i=1}^{N} \eta_{i}\left(\left[\eta_{i} m_{i}+G \phi\left(\tau, \vec{\eta}_{i}(\tau)\right)\right]^{2}+\right.\right. \\
& \left.+\left[\frac{1}{N} \vec{\kappa}_{+}(\tau)+\sqrt{N} \sum_{a=1}^{N-1} \hat{\gamma}_{a i} \vec{\pi}_{a}(\tau)\right]^{2}\right)^{1 / 2} \approx 0, \\
\overrightarrow{\mathcal{H}}_{p}(\tau) & =\vec{\kappa}_{+}+\vec{P}_{\phi} \approx 0,
\end{aligned}
$$

We can now replace the canonical variables $\vec{\eta}_{+}, \vec{\kappa}_{+}, \vec{X}_{\phi}, \vec{P}_{\phi}$ with the following canonical ones

$$
\begin{aligned}
\vec{Y} & =\frac{1}{2}\left(\vec{\eta}_{+}+\vec{X}_{\phi}\right), \\
\overrightarrow{\mathcal{H}}_{p} & =\vec{\kappa}_{+}+\vec{P}_{\phi} \approx 0, \\
\vec{\zeta} & =\vec{\eta}_{+}-\vec{X}_{\phi}, \\
\vec{\pi}_{\zeta} & =\frac{1}{2}\left(\vec{\kappa}_{+}-\vec{P}_{\phi}\right), \\
\vec{\eta}_{+} & =\frac{1}{2} \vec{\zeta}+\vec{Y}, \\
\vec{X}_{\phi} & =-\frac{1}{2} \vec{\zeta}+\vec{Y}, \\
\vec{\kappa}_{+} & =\frac{1}{2} \overrightarrow{\mathcal{H}}_{p}+\vec{\pi}_{\zeta} \approx \vec{\pi}_{\zeta}, \\
\vec{P}_{\phi} & =\frac{1}{2} \overrightarrow{\mathcal{H}}_{p}-\vec{\pi}_{\zeta} \approx-\vec{\pi}_{\zeta} .
\end{aligned}
$$

We see that $\vec{Y}$ is playing the role of the naive "internal" center of mass of the full "particles+field" system: it is the gauge variable conjugate to $\overrightarrow{\mathcal{H}}_{p} \approx 0$

The global relative variables $\vec{\zeta}(\tau), \vec{\pi}_{\zeta}(\tau)$, describe the relative motion of the particle and the field naive centers of mass and rule the action-reaction between the two subsystems.

With the natural gauge-fixings: $\vec{Y} \approx 0, T_{s}-\tau \approx 0$ [so that $\vec{\eta}_{+} \approx \frac{1}{2} \vec{\zeta} \approx-\vec{X}_{\phi}$ ] and the associated Dirac brackets, we get the reduced phase space $\vec{z}_{s}, \vec{k}_{s}, \vec{\zeta}, \vec{\pi}_{\zeta}, \vec{\rho}_{a}(\tau), \vec{\pi}_{a}(\tau), X_{\phi}^{\tau}$, $P_{\phi}^{\tau}, \mathbf{H}\left(T_{s}, \vec{q}\right), \mathbf{K}\left(T_{s}, \vec{q}\right)$ with the evolution in $T_{s} \equiv \tau$ ruled by the Hamiltonian

$$
\begin{aligned}
H & =P_{\phi}^{\tau}+\sum_{i=1}^{N} \eta_{i}\left(\left[\eta_{i} m_{i}+G \phi\left(T_{s}, \frac{1}{2} \vec{\zeta}\left(T_{s}\right)+\frac{1}{\sqrt{N}} \sum_{a=1}^{N-1} \hat{\gamma}_{a i} \vec{\rho}_{a}\left(T_{s}\right)\right)\right]^{2}+\right. \\
& \left.+\left[\frac{1}{N} \vec{\pi}_{\zeta}\left(T_{s}\right)+\sqrt{N} \sum_{a=1}^{N-1} \hat{\gamma}_{a i} \vec{\pi}_{a}\left(T_{s}\right)\right]^{2}\right)^{1 / 2}= \\
& \stackrel{\text { def }}{=} P_{\phi}^{\tau}+\sum_{i=1}^{N} \eta_{i} M_{i},
\end{aligned}
$$




$$
\begin{aligned}
M_{i}^{2}= & \left(\eta_{i} m_{i}+G \phi\right)^{2}+\left[\frac{\vec{\pi}_{\zeta}}{N}+\sqrt{N} \sum_{a=1}^{N-1} \hat{\gamma}_{a i} \vec{\pi}_{a}\right]^{2}, \\
\phi= & \phi\left(T_{s}, \frac{1}{2} \vec{\zeta}\left(T_{s}\right)+\frac{1}{\sqrt{N}} \sum_{a=1}^{N-1} \hat{\gamma}_{a i} \vec{\rho}_{a}\left(T_{s}\right)\right)= \\
= & 2 \int d \tilde{q} \sqrt{F^{\tau}(q) \omega(q) P_{\phi}^{\tau}+F(q) \vec{q} \cdot \vec{\pi}_{\zeta}\left(T_{s}\right)+\mathcal{D}_{\vec{q}} \mathbf{H}\left(T_{s}, \vec{q}\right)} \\
& \cos \left[\vec{q} \cdot\left(\vec{\zeta}\left(T_{s}\right)+\frac{1}{\sqrt{N}} \sum_{a=1}^{N-1} \hat{\gamma}_{a i} \vec{\rho}_{a}\left(T_{s}\right)\right)-\right. \\
- & \left.\omega(q)\left(T_{s}-X_{\phi}^{\tau}\right)+\int d \tilde{k} d \tilde{k}^{\prime} \mathbf{K}\left(T_{s}, \vec{k}\right) \mathcal{G}\left(\vec{k}, \vec{k}^{\prime}\right) \triangle\left(\vec{k}^{\prime}, \vec{q}\right)\right] .
\end{aligned}
$$

The equations of motion for $X_{\phi}^{\tau}, P_{\phi}^{\tau}, \vec{\zeta}(\tau), \vec{\pi}_{\zeta}(\tau), \vec{\rho}_{a}(\tau), \vec{\pi}_{a}(\tau), \mathbf{H}(\tau, \vec{q}), \mathbf{K}(\tau, \vec{q})$ are [remember that $\left.T_{s}-X_{\phi}^{\tau}=-\tilde{X}_{\phi}^{\tau}\right]$

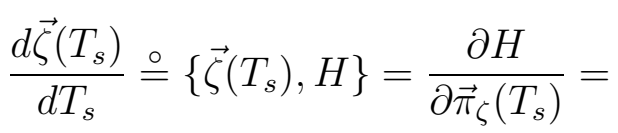

$$
\begin{aligned}
& =\sum_{i=1}^{N} \frac{1}{\eta_{i} M_{i}}\left[\frac{\vec{\pi}_{\zeta}}{N^{2}}+\frac{1}{\sqrt{N}} \sum_{a=1}^{N-1} \hat{\gamma}_{a i} \vec{\pi}_{a}+\right. \\
& +G\left(\eta_{i} m_{i}+G \phi\right) \int d \tilde{q} \frac{F(q) \vec{q}}{\sqrt{F^{\tau}(q) \omega(q) P_{\phi}^{\tau}+F(q) \vec{q} \cdot \vec{\pi}_{\zeta}\left(T_{s}\right)+\mathcal{D}_{\vec{q}} \mathbf{H}\left(T_{s}, \vec{q}\right)}} \\
& \cos \left(\vec{q} \cdot\left(\vec{\zeta}\left(T_{s}\right)+\frac{1}{\sqrt{N}} \sum_{a=1}^{N-1} \hat{\gamma}_{a i} \vec{\rho}_{a i}\left(T_{s}\right)\right)-\right. \\
& \left.\left.-\omega(q)\left(T_{s}-X_{\phi}^{\tau}\right)+\int d \tilde{k} d \tilde{k}^{\prime} \mathbf{K}\left(T_{s}, \vec{k}\right) \mathcal{G}\left(\vec{k}, \vec{k}^{\prime}\right) \triangle\left(\vec{k}^{\prime}, \vec{q}\right)\right)\right], \\
& \frac{d \vec{\pi}_{\zeta}\left(T_{s}\right)}{d T_{s}} \stackrel{\circ}{=}\left\{\vec{\pi}_{\zeta}\left(T_{s}\right), H\right\}=-\frac{\partial H}{\partial \vec{\zeta}\left(T_{s}\right)}= \\
& =2 G \sum_{i=1}^{N} \frac{\eta_{i} m_{i}+G \phi}{\eta_{i} M_{i}} \int d \tilde{q} \vec{q} \sqrt{F^{\tau}(q) \omega(q) P_{\phi}^{\tau}+F(q) \vec{q} \cdot \vec{\pi}_{\zeta}\left(T_{s}\right)+\mathcal{D}_{\vec{q}} \mathbf{H}\left(T_{s}, \vec{q}\right)} \\
& \sin \left(\vec{q} \cdot\left(\vec{\zeta}\left(T_{s}\right)+\frac{1}{\sqrt{N}} \sum_{a=1}^{N-1} \hat{\gamma}_{a i} \vec{\rho}_{a i}\left(T_{s}\right)\right)-\right. \\
& \left.-\omega(q)\left(T_{s}-X_{\phi}^{\tau}\right)+\int d \tilde{k} d \tilde{k}^{\prime} \mathbf{K}\left(T_{s}, \vec{k}\right) \mathcal{G}\left(\vec{k}, \vec{k}^{\prime}\right) \triangle\left(\vec{k}^{\prime}, \vec{q}\right)\right), \\
& \frac{d \vec{\rho}_{a}\left(T_{s}\right)}{d T_{s}} \stackrel{\circ}{=}\left\{\vec{\rho}_{a}\left(T_{s}\right), H\right\}=\frac{\partial H}{\partial \vec{\pi}_{a}\left(T_{s}\right)}= \\
& =\sum_{i=1}^{N} \frac{\hat{\gamma}_{a i}}{\eta_{i} M_{i}}\left(\frac{\vec{\pi}_{\zeta}}{\sqrt{N}}+N \sum_{b=1}^{N-1} \hat{\gamma}_{b i} \vec{\pi}_{b}\right), \\
& \frac{d \vec{\pi}_{a}\left(T_{s}\right)}{d T_{s}} \stackrel{\circ}{=}\left\{\vec{\pi}_{a}\left(T_{s}\right), H\right\}=-\frac{\partial H}{\partial \vec{\rho}_{a}\left(T_{s}\right)}=
\end{aligned}
$$




$$
\begin{aligned}
& =\frac{2 G}{\sqrt{N}} \sum_{i=1}^{N} \hat{\gamma}_{a i} \frac{\eta_{i} m_{i}+G \phi}{\eta_{i} M_{i}} \int d \tilde{q} \vec{q} \sqrt{F^{\tau}(q) \omega(q) P_{\phi}^{\tau}+F(q) \vec{q} \cdot \vec{\pi}_{\zeta}\left(T_{s}\right)+\mathcal{D}_{\vec{q}} \mathbf{H}\left(T_{s}, \vec{q}\right)} \\
& \sin \left(\vec{q} \cdot\left(\vec{\zeta}\left(T_{s}\right)+\frac{1}{\sqrt{N}} \sum_{a=1}^{N-1} \hat{\gamma}_{a i} \vec{\rho}_{a i}\left(T_{s}\right)\right)-\right. \\
& \left.-\omega(q)\left(T_{s}-X_{\phi}^{\tau}\right)+\int d \tilde{k} d \tilde{k}^{\prime} \mathbf{K}\left(T_{s}, \vec{k}\right) \mathcal{G}\left(\vec{k}, \vec{k}^{\prime}\right) \triangle\left(\vec{k}^{\prime}, \vec{q}\right)\right), \\
& \frac{d X_{\phi}^{\tau}}{d T_{s}} \stackrel{\circ}{=}\left\{X_{\phi}^{\tau}, H\right\}=-\frac{\partial H}{\partial P_{\phi}^{\tau}}= \\
& =-1+G \sum_{i=1}^{N} \frac{\eta_{i} m_{i}+G \phi}{\eta_{i} M_{i}} \int d \tilde{q} \frac{F^{\tau}(q) \omega(q)}{\sqrt{F^{\tau}(q) \omega(q) P_{\phi}^{\tau}+F(q) \vec{q} \cdot \vec{\pi}_{\zeta}\left(T_{s}\right)+\mathcal{D}_{\vec{q}} \mathbf{H}\left(T_{s}, \vec{q}\right)}} \\
& \cos \left(\vec{q} \cdot\left(\vec{\zeta}\left(T_{s}\right)+\frac{1}{\sqrt{N}} \sum_{a=1}^{N-1} \hat{\gamma}_{a i} \vec{\rho}_{a i}\left(T_{s}\right)\right)-\right. \\
& \left.-\omega(q)\left(T_{s}-X_{\phi}^{\tau}\right)+\int d \tilde{k} d \tilde{k}^{\prime} \mathbf{K}\left(T_{s}, \vec{k}\right) \mathcal{G}\left(\vec{k}, \vec{k}^{\prime}\right) \triangle\left(\vec{k}^{\prime}, \vec{q}\right)\right), \\
& \frac{d P_{\phi}^{\tau}}{d T_{s}} \stackrel{\circ}{=}\left\{P_{\phi}^{\tau}, H\right\}=\frac{\partial H}{\partial X_{\phi}^{\tau}}= \\
& =-2 G \sum_{i=1}^{N} \frac{\eta_{i} m_{i}+G \phi}{\eta_{i} M_{i}} \int d \tilde{q} \omega(q) \sqrt{F^{\tau}(q) \omega(q) P_{\phi}^{\tau}+F(q) \vec{q} \cdot \vec{\pi}_{\zeta}\left(T_{s}\right)+\mathcal{D}_{\vec{q}} \mathbf{H}\left(T_{s}, \vec{q}\right)} \\
& \sin \left(\vec{q} \cdot\left(\vec{\zeta}\left(T_{s}\right)+\frac{1}{\sqrt{N}} \sum_{a=1}^{N-1} \hat{\gamma}_{a i} \vec{\rho}_{a i}\left(T_{s}\right)\right)-\right. \\
& \left.-\omega(q)\left(T_{s}-X_{\phi}^{\tau}\right)+\int d \tilde{k} d \tilde{k}^{\prime} \mathbf{K}\left(T_{s}, \vec{k}\right) \mathcal{G}\left(\vec{k}, \vec{k}^{\prime}\right) \triangle\left(\vec{k}^{\prime}, \vec{q}\right)\right), \\
& \left.\frac{\partial \mathbf{H}\left(T_{s}, \vec{q}\right)}{\partial T_{s}} \stackrel{\circ}{=} \mathbf{H}\left(T_{s}, \vec{q}\right), H\right\}=\frac{\delta H}{\delta \mathbf{K}\left(T_{s}, \vec{q}\right)}= \\
& =-2 G \sum_{i=1}^{N} \frac{\eta_{i} m_{i}+G \phi}{\eta_{i} M_{i}} \int d \tilde{k} d \tilde{k}^{\prime} \mathcal{G}\left(\vec{q}, \vec{k}^{\prime}\right) \triangle\left(\vec{k}^{\prime}, \vec{k}\right) \\
& \sqrt{F^{\tau}(q) \omega(q) P_{\phi}^{\tau}+F(q) \vec{q} \cdot \vec{\pi}_{\zeta}\left(T_{s}\right)+\mathcal{D}_{\vec{q}} \mathbf{H}\left(T_{s}, \vec{q}\right)} \\
& \sin \left(\vec{k} \cdot\left(\vec{\zeta}\left(T_{s}\right)+\frac{1}{\sqrt{N}} \sum_{a=1}^{N-1} \hat{\gamma}_{a i} \vec{\rho}_{a i}\left(T_{s}\right)\right)-\right. \\
& \left.-\omega(k)\left(T_{s}-X_{\phi}^{\tau}\right)+\int d \tilde{k}_{1} d \tilde{k}_{2} \mathbf{K}\left(T_{s}, \vec{k}_{1}\right) \mathcal{G}\left(\vec{k}_{1}, \vec{k}_{2}\right) \triangle\left(\vec{k}_{2}, \vec{k}\right)\right) \text {, } \\
& \frac{\partial \mathbf{K}\left(T_{s}, \vec{q}\right)}{\partial T_{s}} \stackrel{\circ}{=}\left\{\mathbf{K}\left(T_{s}, \vec{q}\right), H\right\}=-\frac{\delta H}{\delta \mathbf{H}\left(T_{s}, \vec{q}\right)}= \\
& =-2 G \sum_{i=1}^{N} \frac{\eta_{i} m_{i}+G \phi}{\eta_{i} M_{i}} \\
& \mathcal{D}_{\vec{q}}\left[\operatorname { c o s } \left(\vec{q} \cdot\left(\vec{\zeta}\left(T_{s}\right)+\frac{1}{\sqrt{N}} \sum_{a=1}^{N-1} \hat{\gamma}_{a i} \vec{\rho}_{a i}\left(T_{s}\right)\right)-\right.\right.
\end{aligned}
$$




$$
\begin{array}{r}
\left.-\omega(q)\left(T_{s}-X_{\phi}^{\tau}\right)+\int d \tilde{k} d \tilde{k}^{\prime} \mathbf{K}\left(T_{s}, \vec{k}\right) \mathcal{G}\left(\vec{k}, \vec{k}^{\prime}\right) \triangle\left(\vec{k}^{\prime}, \vec{q}\right)\right) \\
\left.\left(\sqrt{F^{\tau}(q) \omega(q) P_{\phi}^{\tau}+F(q) \vec{q} \cdot \vec{\pi}_{\zeta}\left(T_{s}\right)+\mathcal{D}_{\vec{q}} \mathbf{H}\left(T_{s}, \vec{q}\right)}\right)^{-1}\right] .
\end{array}
$$

When the decomposition into center-of-mass and relative variables will be available for the transverse electromagnetic field in the Coulomb gauge, one will be able to make a similar treatment of the isolated system consisting of $\mathrm{N}$ charged particles (with Grassmann-valued electric charges) plus the electromagnetic field [1, 8 . 


\section{CHARGED KLEIN-GORDON FIELD.}

\section{A. On spacelike hypersurfaces.}

Following Ref. [8], let us now consider a charged Klein-Gordon field: $\phi=\frac{1}{\sqrt{2}}\left(\phi_{1}+i \phi_{2}\right)$, $\phi^{*}=\frac{1}{\sqrt{2}}\left(\phi_{1}-i \phi_{2}\right)\left[\phi_{1}=\frac{1}{\sqrt{2}}\left(\phi+\phi^{*}\right), \phi_{2}=\frac{-i}{\sqrt{2}}\left(\phi-\phi^{*}\right)\right]$, whose action is

$$
\begin{aligned}
S= & \int d \tau d^{3} \sigma N(\tau, \vec{\sigma}) \sqrt{\gamma(\tau, \vec{\sigma})}\left(g^{\tau \tau} \dot{\phi}^{*} \dot{\phi}+\right. \\
& \left.+g^{\tau \check{r}}\left[\dot{\phi}^{*} \partial_{\check{r}} \phi+\partial_{\check{r}} \phi^{*} \dot{\phi}\right]+g^{\check{r} \check{s}} \partial_{\check{r}} \phi^{*} \partial_{\check{s}} \phi-m^{2} \phi^{*} \phi\right)(\tau, \vec{\sigma})= \\
& =\int d \tau d^{3} \sigma \sqrt{\gamma(\tau, \vec{\sigma})}\left(\frac{1}{N}\left[\dot{\phi}^{*}-N^{\check{r}} \partial_{\check{r}} \phi^{*}\right]\left[\dot{\phi}-N^{\check{s}} \partial_{\check{s}} \phi\right]+\right. \\
+ & \left.N\left[\gamma^{\check{r} \check{s}} \partial_{\check{r}} \phi^{*} \partial_{\check{s}} \phi-m^{2} \phi^{*} \phi\right]\right)(\tau, \vec{\sigma}) .
\end{aligned}
$$

The canonical momenta are

$$
\begin{aligned}
\pi_{\phi}(\tau, \vec{\sigma})= & \frac{\partial L}{\partial \partial_{\tau} \phi(\tau, \vec{\sigma})}=\frac{1}{\sqrt{2}}\left(\pi_{1}-i \pi_{2}\right)(\tau, \vec{\sigma})= \\
= & \frac{\sqrt{\gamma(\tau, \vec{\sigma})}}{N(\tau, \vec{\sigma})}\left[\dot{\phi}^{*}-N^{\check{r}} \partial_{\check{r}} \phi^{*}\right](\tau, \vec{\sigma}), \\
\pi_{\phi^{*}}(\tau, \vec{\sigma})= & \frac{\partial L}{\partial \partial_{\tau} \phi^{*}(\tau, \vec{\sigma})}=\frac{1}{\sqrt{2}}\left(\pi_{1}+i \pi_{2}\right)(\tau, \vec{\sigma})= \\
= & \frac{\sqrt{\gamma(\tau, \vec{\sigma})}}{N(\tau, \vec{\sigma})}\left[\dot{\phi}-N^{\check{r}} \partial_{\check{r}} \phi\right](\tau, \vec{\sigma}), \\
\rho_{\mu}(\tau, \vec{\sigma})= & -\frac{\partial L}{\partial \partial_{\tau} z^{\mu}(\tau, \vec{\sigma})}= \\
= & l_{\mu}(\tau, \vec{\sigma})\left(\frac{\pi_{\phi} \pi_{\phi^{*}}}{\sqrt{\gamma}}-\sqrt{\gamma}\left[\gamma^{\check{r} \check{s}} \partial_{\check{r}} \phi^{*} \partial_{\check{s}} \phi-m^{2} \phi^{*} \phi\right]\right)(\tau, \vec{\sigma})+ \\
& +z_{\check{s} \mu}(\tau, \vec{\sigma}) \gamma^{\check{r} \check{s}}(\tau, \vec{\sigma})\left(\pi_{\phi^{*}} \partial_{\check{r}} \phi^{*}+\pi_{\phi} \partial_{\check{r}} \phi\right)(\tau, \vec{\sigma}) .
\end{aligned}
$$

Therefore, we have the following primary constraints

$$
\begin{aligned}
\mathcal{H}_{\mu}(\tau, \vec{\sigma})= & \rho_{\mu}(\tau, \vec{\sigma})- \\
& -l_{\mu}(\tau, \vec{\sigma})\left(\frac{\pi_{\phi} \pi_{\phi^{*}}}{\sqrt{\gamma}}-\sqrt{\gamma}\left[\gamma^{\check{r} \check{s}} \partial_{\check{r}} \phi^{*} \partial_{\check{s}} \phi-m^{2} \phi^{*} \phi\right]\right)(\tau, \vec{\sigma})+ \\
& +z_{\check{s} \mu}(\tau, \vec{\sigma}) \gamma^{\check{r} \check{s}}(\tau, \vec{\sigma})\left(\pi_{\phi^{*}} \partial_{\check{r}} \phi^{*}+\pi_{\phi} \partial_{\check{r}} \phi\right)(\tau, \vec{\sigma}) \approx 0,
\end{aligned}
$$

and the following Dirac Hamiltonian

$$
H_{D}=\int d^{3} \sigma \lambda^{\mu}(\tau, \vec{\sigma}) \mathcal{H}_{\mu}(\tau, \vec{\sigma})
$$

By using the Poisson brackets

$$
\begin{aligned}
\left\{z^{\mu}(\tau, \vec{\sigma}), \rho_{\nu}\left(\tau, \vec{\sigma}^{\prime}\right)\right\} & =\eta_{\nu}^{\mu} \delta^{3}\left(\vec{\sigma}-\vec{\sigma}^{\prime}\right), \\
\left\{\phi(\tau, \vec{\sigma}), \pi_{\phi}\left(\tau, \vec{\sigma}^{\prime}\right)\right\} & =\left\{\phi^{*}(\tau, \vec{\sigma}), \pi_{\phi^{*}}\left(\tau, \vec{\sigma}^{\prime}\right)\right\}=\delta^{3}\left(\vec{\sigma}-\vec{\sigma}^{\prime}\right),
\end{aligned}
$$


we find that the time constancy of the primary constraints implies the existence of no secondary constraint. The constraints turn out to be first class.

After the restriction to spacelike hyperplanes $z^{\mu}(\tau, \vec{\sigma})=x_{s}^{\mu}(\tau)+b_{\check{r}}^{\mu}(\tau) \sigma^{\check{r}}$, the constraints are reduced to the following ones

$$
\begin{aligned}
\tilde{\mathcal{H}}^{\mu}(\tau)= & \int d^{3} \sigma \mathcal{H}^{\mu}(\tau, \vec{\sigma})= \\
= & p_{s}^{\mu}-l^{\mu}\left(\int d^{3} \sigma\left[\pi_{\phi^{*}} \pi_{\phi}+\vec{\partial} \phi^{*} \cdot \vec{\partial} \phi+m^{2} \phi^{*} \phi\right](\tau, \vec{\sigma})\right)- \\
- & b_{\check{r}}^{\mu}(\tau)\left\{\int d^{3} \sigma\left[\pi_{\phi^{*}} \partial_{\check{r}} \phi^{*}+\pi_{\phi} \partial_{\check{r}} \phi\right](\tau, \vec{\sigma})\right\} \approx 0 \\
\tilde{\mathcal{H}}^{\mu \nu}(\tau)= & b_{\check{r}}^{\mu}(\tau) \int d^{3} \sigma \sigma^{\check{r}} \mathcal{H}^{\nu}(\tau, \vec{\sigma})-b_{\check{r}}^{\nu}(\tau) \int d^{3} \sigma \sigma^{\check{r}} \mathcal{H}^{\mu}(\tau, \vec{\sigma})= \\
= & S_{s}^{\mu \nu}-\left(b_{\check{r}}^{\mu}(\tau) l^{\nu}-b_{\check{r}}^{\nu}(\tau) l^{\mu}\right) \\
& \int d^{3} \sigma \sigma^{\check{r}}\left[\pi_{\phi^{*}} \pi_{\phi}+\vec{\partial} \phi^{*} \cdot \vec{\partial} \phi+m^{2} \phi^{*} \phi\right](\tau, \vec{\sigma})+ \\
+ & \left(b_{\check{r}}^{\mu}(\tau) b_{\check{s}}^{\nu}(\tau)-b_{\check{r}}^{\nu}(\tau) b_{\check{s}}^{\mu}(\tau)\right) \\
& \int d^{3} \sigma \sigma^{\check{r}}\left[\pi_{\phi^{*}} \partial_{\check{s}} \phi^{*}+\pi_{\phi} \partial_{\check{s}} \phi\right](\tau, \vec{\sigma}) \approx 0 .
\end{aligned}
$$

The only surviving constraints on the Wigner hyperplanes [with the reduced canonical variables $\tilde{x}_{s}^{\mu}(\tau), p_{s}^{\mu}, \phi(\tau, \vec{\sigma}), \pi_{\phi}(\tau, \vec{\sigma})=\dot{\phi}^{*}(\tau, \vec{\sigma}), \phi^{*}(\tau, \vec{\sigma}), \pi_{\phi^{*}}(\tau, \vec{\sigma})=\dot{\phi}(\tau, \vec{\sigma})$, satisfying standard Dirac brackets] are

$$
\begin{aligned}
\mathcal{H}(\tau) & =\epsilon_{s}-\int d^{3} \sigma\left[\pi_{\phi^{*}} \pi_{\phi}+\vec{\partial} \phi^{*} \cdot \vec{\partial} \phi+m^{2} \phi^{*} \phi\right](\tau, \vec{\sigma}) \approx 0, \\
\overrightarrow{\mathcal{H}}_{p}(\tau) & =\int d^{3} \sigma\left[\pi_{\phi^{*}} \vec{\partial} \phi^{*}+\pi_{\phi} \vec{\partial} \phi\right](\tau, \vec{\sigma}) \approx 0 .
\end{aligned}
$$

The Lorentz generators have the form of Eq.(2.9) with the spin tensor

$$
\bar{S}_{s}^{r s} \equiv S_{\phi}^{r s}=\left.J_{\phi}^{r s}\right|_{\vec{P}_{\phi}=0}=\int d^{3} \sigma\left(\sigma^{r}\left[\pi_{\phi^{*}} \partial^{s} \phi^{*}+\pi_{\phi} \partial^{s} \phi\right](\tau, \vec{\sigma})-(r \leftrightarrow s)\right) .
$$

With the gauge fixing $\chi=T_{s}-\tau \approx 0$ the final Hamiltonian becomes

$$
\begin{aligned}
& H_{R}=M_{\phi}-\vec{\lambda}\left(T_{s}\right) \cdot \overrightarrow{\mathcal{H}}_{p}\left(T_{s}\right), \\
& M_{\phi}=\int d^{3} \sigma\left[\pi_{\phi^{*}} \pi_{\phi}+\vec{\partial} \phi^{*} \cdot \vec{\partial} \phi+m^{2} \phi^{*} \phi\right]\left(T_{s}, \vec{\sigma}\right)
\end{aligned}
$$

\section{B. Collective and relative variables.}

A priori we have the following two possibilities for the description of the complex KleinGordon field $\phi(\tau, \vec{\sigma})$ in terms of a Fourier transform

$$
\begin{aligned}
\phi(\tau, \vec{\sigma}) & =\frac{1}{\sqrt{2}}\left[\phi_{1}(\tau, \vec{\sigma})+i \phi_{2}(\tau, \vec{\sigma})\right]= \\
& =\frac{1}{\sqrt{2}} \int d \tilde{q}\left(\left[a_{1}(\tau, \vec{q}) e^{-i(\omega(q) \tau-\vec{q} \cdot \vec{\sigma})}+a_{1}^{*}(\tau, \vec{q}) e^{+i(\omega(q) \tau-\vec{q} \cdot \vec{\sigma})}\right]+\right.
\end{aligned}
$$




$$
\begin{aligned}
& \left.+i\left[a_{2}(\tau, \vec{q}) e^{-i(\omega(q) \tau-\vec{q} \cdot \vec{\sigma})}+a_{2}^{*}(\tau, \vec{q}) e^{+i(\omega(q) \tau-\vec{q} \cdot \vec{\sigma})}\right]\right)= \\
& =\int d \tilde{q}\left[a(\tau, \vec{q}) e^{-i(\omega(q) \tau-\vec{q} \cdot \vec{\sigma})}+b^{*}(\tau, \vec{q}) e^{+i(\omega(q) \tau-\vec{q} \cdot \vec{\sigma})}\right], \\
& \phi^{*}(\tau, \vec{\sigma})=\frac{1}{\sqrt{2}}\left[\phi_{1}(\tau, \vec{\sigma})-i \phi_{2}(\tau, \vec{\sigma})\right]= \\
& =\frac{1}{\sqrt{2}} \int d \tilde{q}\left(\left[a_{1}(\tau, \vec{q}) e^{-i(\omega(q) \tau-\vec{q} \cdot \vec{\sigma})}+a_{1}^{*}(\tau, \vec{q}) e^{i(\omega(q) \tau-\vec{q} \cdot \vec{\sigma})}\right]-\right. \\
& \left.-i\left[a_{2}(\tau, \vec{q}) e^{-i(\omega(q) \tau-\vec{q} \cdot \vec{\sigma})}+a_{2}^{*}(\tau, \vec{q}) e^{+i(\omega(q) \tau-\vec{q} \cdot \vec{\sigma})}\right]\right)= \\
& =\int d \tilde{q}\left[b(\tau, \vec{q}) e^{-i(\omega(q) \tau-\vec{q} \cdot \vec{\sigma})}+a^{*}(\tau, \vec{q}) e^{+i(\omega(q) \tau-\vec{q} \cdot \vec{\sigma})}\right], \\
& \pi(\tau, \vec{\sigma})=\frac{1}{\sqrt{2}}\left[\pi_{1}(\tau, \vec{\sigma})-i \pi_{2}(\tau, \vec{\sigma})\right]= \\
& =\frac{-i}{\sqrt{2}} \int d \tilde{q}\left(\left[a_{1}(\tau, \vec{q}) e^{-i(\omega(q) \tau-\vec{q} \cdot \vec{\sigma})}-a_{1}^{*}(\tau, \vec{q}) e^{+i(\omega(q) \tau-\vec{q} \cdot \vec{\sigma})}\right]-\right. \\
& \left.-i\left[a_{2}(\tau, \vec{q}) e^{-i(\omega(q) \tau-\vec{q} \cdot \vec{\sigma})}-a_{2}^{*}(\tau, \vec{q}) e^{+i(\omega(q) \tau-\vec{q} \cdot \vec{\sigma})}\right]\right)= \\
& =-i \int d \tilde{q} \omega(q)\left[b(\tau, \vec{q}) e^{-i(\omega(q) \tau-\vec{q} \cdot \vec{\sigma})}-a^{*}(\tau, \vec{q}) e^{+i(\omega(\vec{q}) \tau-\vec{q} \cdot \vec{\sigma})}\right] \text {, } \\
& \pi^{*}(\tau, \vec{\sigma})=\frac{1}{\sqrt{2}}\left[\pi_{1}(\tau, \vec{\sigma})+i \pi_{2}(\tau, \vec{\sigma})\right]= \\
& =\frac{-i}{\sqrt{2}} \int d \tilde{q}\left(\left[a_{1}(\tau, \vec{q}) e^{-i(\omega(q) \tau-\vec{q} \cdot \vec{\sigma})}-a_{1}^{*}(\tau, \vec{q}) e^{+i(\omega(q) \tau-\vec{q} \cdot \vec{\sigma})}\right]+\right. \\
& \left.+i\left[a_{2}(\tau, \vec{q}) e^{-i(\omega(q) \tau-\vec{q} \cdot \vec{\sigma})}-a_{2}^{*}(\tau, \vec{q}) e^{+i(\omega(q) \tau-\vec{q} \cdot \vec{\sigma})}\right]\right)= \\
& =-i \int d \tilde{q} \omega(q)\left[a(\tau, \vec{q}) e^{-i(\omega(\vec{q}) \tau-\vec{q} \cdot \vec{\sigma})}-b^{*}(\tau, \vec{q}) e^{+i(\omega(q) \tau-\vec{q} \cdot \vec{\sigma})}\right], \\
& a_{i}(\tau, \vec{q})=\sqrt{I_{i}(\tau, \vec{q})} e^{i \varphi_{i}(\tau, \vec{q})}, \quad i=1,2, \\
& a(\tau, \vec{q})=\frac{1}{\sqrt{2}}\left[a_{1}(\tau, \vec{q})+i a_{2}(\tau, \vec{q})\right]=\frac{1}{\sqrt{2}}\left[\sqrt{I_{1}} e^{i \varphi_{1}}+i \sqrt{I_{2}} e^{i \varphi_{2}}\right](\tau, \vec{q})= \\
& =\int d^{3} \sigma\left[\omega(q) \phi(\tau, \vec{\sigma})+i \pi^{*}(\tau, \vec{\sigma})\right] e^{i(\omega(q) \tau-\vec{q} \cdot \vec{\sigma})}=\sqrt{I_{a}(\tau, \vec{q})} e^{i \varphi_{a}(\tau, \vec{q})}, \\
& b(\tau, \vec{q})=\frac{1}{\sqrt{2}}\left[a_{1}(\tau, \vec{q})-i a_{2}(\tau, \vec{q})\right]=\frac{1}{\sqrt{2}}\left[\sqrt{I_{1}} e^{i \varphi_{1}}-i \sqrt{I_{2}} e^{i \varphi_{2}}\right](\tau, \vec{q})= \\
& =\int d^{3} \sigma\left[\omega(q) \phi^{*}(\tau, \vec{\sigma})+i \pi(\tau, \vec{\sigma})\right] e^{i(\omega(q) \tau-\vec{q} \cdot \vec{\sigma})}=\sqrt{I_{b}(\tau, \vec{q})} e^{i \varphi_{b}(\tau, \vec{q})}, \\
& a^{*}(\tau, \vec{q})=\frac{1}{\sqrt{2}}\left[a_{1}^{*}(\tau, \vec{q})-i a_{2}^{*}(\tau, \vec{q})\right]= \\
& =\int d^{3} \sigma\left[\omega(q) \phi^{*}(\tau, \vec{\sigma})-i \pi(\tau, \vec{\sigma})\right] e^{-i(\omega(q) \tau-\vec{q} \cdot \vec{\sigma})}, \\
& b^{*}(\tau, \vec{q})=\frac{1}{\sqrt{2}}\left[a_{1}^{*}(\tau, \vec{q})+i a_{2}^{*}(\tau, \vec{q})\right]= \\
& =\int d^{3} \sigma\left[\omega(q) \phi(\tau, \vec{\sigma})-i \pi^{*}(\tau, \vec{\sigma})\right] e^{-i(\omega(q) \tau-\vec{q} \cdot \vec{\sigma})},
\end{aligned}
$$




$$
\begin{aligned}
& I_{i}(\tau, \vec{q})=a_{i}^{*}(\tau, \vec{q}) a_{i}(\tau, \vec{q}), \\
& I_{a}(\tau, \vec{q})=a^{*}(\tau, \vec{q}) a(\tau, \vec{q})=\frac{1}{2}\left[a_{1}^{*} a_{1}+a_{2}^{*} a_{2}+i\left(a_{1}^{*} a_{2}-a_{2}^{*} a_{1}\right)\right](\tau, \vec{q}), \\
& I_{b}(\tau, \vec{q})=b^{*}(\tau, \vec{q}) b(\tau, \vec{q})=\frac{1}{2}\left[a_{1}^{*} a_{1}+a_{2}^{*} a_{2}-i\left(a_{1}^{*} a_{2}-a_{2}^{*} a_{1}\right)\right], \\
& \left(I_{a}+I_{b}\right) \quad(\tau, \vec{q})=\left[I_{1}+I_{2}\right](\tau, \vec{q}), \\
& \left(I_{a}-I_{b}\right) \quad(\tau, \vec{q})=i\left[a_{1}^{*} a_{2}-a_{2}^{*} a_{1}\right](\tau, \vec{q}), \\
& N_{i \phi}=\int d \tilde{q} a_{i}^{*}(\tau, \vec{q}) a_{i}(\tau, \vec{q})=\int d \tilde{q} I_{i}(\tau, \vec{\sigma}), \\
& N_{a \phi}=\int d \tilde{q} a^{*}(\tau, \vec{q}) a(\tau, \vec{q})=\frac{1}{2}\left[N_{1 \phi}+N_{2 \phi}\right]+\frac{i}{2} \int d \tilde{q}\left[a_{1}^{*} a_{2}-a_{2}^{*} a_{1}\right](\tau, \vec{q})= \\
& =\int d \tilde{q} I_{a}(\tau, \vec{q})= \\
& =\frac{1}{2} \int d^{3} \sigma\left[\pi_{\phi^{*}} \frac{1}{\sqrt{m^{2}+\triangle}} \pi+\phi^{*} \sqrt{m^{2}+\triangle} \phi+i\left(\pi_{\phi^{*}} \phi^{*}-\pi \phi\right)\right](\tau, \vec{\sigma}), \\
& N_{b \phi}=\int d \tilde{q} b^{*}(\tau, \vec{q}) b(\tau, \vec{q})=\frac{1}{2}\left[N_{1 \phi}+N_{2 \phi}\right]-\frac{i}{2} \int d \tilde{q}\left[a_{1}^{*} a_{2}-a_{2}^{*} a_{1}\right](\tau, \vec{q})= \\
& +\int d \tilde{q} I_{b}(\tau, \vec{q})= \\
& =\frac{1}{2} \int d^{3} \sigma\left[\pi_{\phi^{*}} \frac{1}{\sqrt{m^{2}+\triangle}} \pi+\phi^{*} \sqrt{m^{2}+\triangle} \phi-i\left(\pi_{\phi^{*}} \phi^{*}-\pi \phi\right)\right](\tau, \vec{\sigma}), \\
& N_{\phi}=N_{1 \phi}+N_{2 \phi}=N_{a \phi}+N_{b \phi}= \\
& =\int d^{3} \sigma\left[\pi_{\phi^{*}} \frac{1}{\sqrt{m^{2}+\triangle}} \pi+\phi^{*} \sqrt{m^{2}+\triangle} \phi\right](\tau, \vec{\sigma})
\end{aligned}
$$

However, the description of the conserved electric charge of the Klein-Gordon field privileges the use of the Fourier coefficients $a(\tau, \vec{q}), b(\tau, \vec{q})$ rather than of the $a_{i}(\tau, \vec{q})$ 's, because we have

$$
q_{\phi}=i \int d^{3} \sigma\left[\pi_{\phi^{*}} \phi^{*}-\pi \phi\right](\tau, \vec{\sigma})=N_{b \phi}-N_{a \phi}, \quad \frac{d q_{\phi}}{d \tau} \stackrel{\circ}{=} 0
$$

on the Wigner hyperplanes the conservation $\partial_{\mu} J_{\phi}^{\mu} \stackrel{\circ}{=} 0$ of the electromagnetic current $J_{\phi}^{\mu}(x)=$ $-i\left[\partial^{\mu} \tilde{\phi}^{*} \tilde{\phi}-\tilde{\phi}^{*} \partial^{\mu} \tilde{\phi}\right](x)$ is replaced by the existence of the Hamiltonian constant of motion $q_{\phi}$.

The Fourier coefficients $a_{i}(\tau, \vec{\sigma}), \mathrm{i}=1,2$, correspond to the description of the field as two real Klein-Gordon fields $\phi_{i}$. Instead, as shown in Appendix D, the Fourier coefficients "a" and "b" correspond to two Klein-Gordon fields $\phi_{a}, \phi_{b}$, with positive $\left(\phi_{a}\right)$ or negative $\left(\phi_{b}\right)$ energy and electric charge, which are nonlocal combinations of $\phi$ and $\pi_{\phi^{*}}$.

The total 4-momentum of the Klein-Gordon field may be described either in terms of the "a" and "b" or of the " $a_{i}$ "

$$
\begin{aligned}
P_{\phi}^{\tau} & =\int d \tilde{q} \omega(q)\left[I_{1}+I_{2}\right](\tau, \vec{q})=P_{1 \phi}^{\tau}(\tau)+P_{2 \phi}^{\tau}(\tau)= \\
& =\int d \tilde{q} \omega(q)\left[I_{a}+I_{b}\right](\tau, \vec{q})=P_{a \phi}^{\tau}(\tau)+P_{b \phi}^{\tau}(\tau)= \\
& =\int d^{3} \sigma\left[\pi_{\phi^{*}} \pi_{\phi}+\vec{\partial} \phi^{*} \cdot \vec{\partial} \phi+m^{2} \phi^{*} \phi\right](\tau, \vec{\sigma}),
\end{aligned}
$$




$$
\begin{aligned}
\vec{P}_{\phi} & =\int d \tilde{q} \vec{q}\left[I_{1}+I_{2}\right](\tau, \vec{q})=\vec{P}_{1 \phi}(\tau)+\vec{P}_{2 \phi}(\tau)= \\
& =\int d \tilde{q} \vec{q}\left[I_{a}+I_{b}\right](\tau, \vec{q})=\vec{P}_{a \phi}(\tau)+\vec{P}_{b \phi}(\tau)= \\
& =\int d^{3} \sigma\left[\pi_{\phi^{*}} \vec{\partial} \phi^{*}+\pi_{\phi} \vec{\partial} \phi\right](\tau, \vec{\sigma}),
\end{aligned}
$$

but it is possible to define a global "relative 4-momentum" of the field only in terms of the "a" and "b"

$$
\begin{aligned}
Q_{\phi}^{\tau}(\tau) & =\frac{1}{2} \int d \tilde{q} \omega(q)\left[I_{a}-I_{b}\right](\tau, \vec{q})=P_{a \phi}^{\tau}(\tau)-P_{b \phi}^{\tau}(\tau)= \\
& =\frac{i}{2} \int d^{3} \sigma\left[\pi_{\phi^{*}} \sqrt{m^{2}+\triangle \phi^{*}}-\pi \sqrt{m^{2}+\triangle \phi}\right](\tau, \vec{\sigma}) \neq \\
& \neq \frac{1}{2} \int d \tilde{q} \omega(q)\left[I_{1}-I_{2}\right](\tau, \vec{q})=P_{1 \phi}^{\tau}(\tau)-P_{2 \phi}^{\tau}(\tau), \\
\vec{Q}_{\phi}(\tau) & =\frac{1}{2} \int d \tilde{q} \vec{q}\left[I_{a}-I_{b}\right](\tau, \vec{q})=\vec{P}_{a \phi}(\tau)-\vec{P}_{b \phi}(\tau)= \\
& =-\frac{i}{2} \int d^{3} \sigma\left[\pi_{\phi^{*}} \frac{\vec{\partial}}{\sqrt{m^{2}+\triangle}} \pi-\phi^{*} \sqrt{m^{2}+\triangle} \vec{\partial} \phi\right](\tau, \vec{\sigma}) \neq \\
& \neq \frac{1}{2} \int d \tilde{q} \vec{q}\left[I_{1}-I_{2}\right](\tau, \vec{q})=\vec{P}_{1 \phi}(\tau)-\vec{P}_{2 \phi}(\tau), \\
P_{a \phi}^{\tau} & =\frac{1}{2}\left(P_{\phi}^{\tau}+Q_{\phi}^{\tau}\right), \quad \vec{P}_{a \phi}=\frac{1}{2}\left(\vec{P}_{\phi}+\vec{Q}_{\phi}\right), \\
P_{b \phi}^{\tau} & =\frac{1}{2}\left(P_{\phi}^{\tau}-Q_{\phi}^{\tau}\right), \quad \vec{P}_{b \phi}=\frac{1}{2}\left(\vec{P}_{\phi}-\vec{Q}_{\phi}\right),
\end{aligned}
$$

The two partial centers of phase have coordinates

$$
\begin{aligned}
& X_{a \phi}^{\tau}=\int d \tilde{q} \omega(q) F^{\tau}(q) \varphi_{a}(\tau, \vec{q})=\frac{1}{2 i} \int d \tilde{q} \omega(q) F^{\tau}(q) \ln \frac{a(\tau, \vec{q})}{a^{*}(\tau, \vec{q})}=\tau+ \\
& +\frac{1}{2 \pi i m} \int d^{3} q \frac{e^{-\frac{4 \pi}{m^{2}} q^{2}}}{q^{2} \omega(q)} \ln \left[\frac{\omega(q) \int d^{3} \sigma e^{i \vec{q} \cdot \vec{\sigma}} \phi(\tau, \vec{\sigma})+i \int d^{3} \sigma e^{i \vec{q} \cdot \vec{\sigma}} \pi_{\phi^{*}}(\tau, \vec{\sigma})}{\omega(q) \int d^{3} \sigma e^{-i \vec{q} \cdot \vec{\sigma}} \phi^{*}(\tau, \vec{\sigma})-i \int d^{3} \sigma e^{-i \vec{q} \cdot \vec{\sigma}} \pi(\tau, \vec{\sigma})}\right]= \\
& \stackrel{\text { def }}{=} \tau+\tilde{X}_{a \phi}^{\tau} \\
& \vec{X}_{a \phi}=\int d \tilde{q} \vec{q} F(q) \varphi_{a}(\tau, \vec{q})=\frac{1}{2 i} \int d \tilde{q} \vec{q} F(q) \ln \frac{a(\tau, \vec{q})}{a^{*}(\tau, \vec{q})}= \\
& =\frac{2 i}{\pi m} \int d^{3} q \frac{\vec{q}}{q^{4}} e^{-\frac{4 \pi}{m^{2}} q^{2}} \ln \left[\frac{\sqrt{m^{2}+q^{2}} \int d^{3} \sigma e^{i \vec{q} \cdot \vec{\sigma}} \phi(\tau, \vec{\sigma})+i \int d^{3} \sigma e^{i \vec{q} \cdot \vec{\sigma}} \pi_{\phi^{*}}(\tau, \vec{\sigma})}{\sqrt{m^{2}+q^{2}} \int d^{3} \sigma e^{-i \vec{q} \cdot \vec{\sigma}} \phi^{*}(\tau, \vec{\sigma})-i \int d^{3} \sigma e^{-i \vec{q} \cdot \vec{\sigma}} \pi(\tau, \vec{\sigma})}\right], \\
& X_{b \phi}^{\tau}=\int d \tilde{q} \omega(q) F^{\tau}(q) \varphi_{b}(\tau, \vec{q})=\frac{1}{2 i} \int d \tilde{q} \omega(q) F^{\tau}(q) \ln \frac{b(\tau, \vec{q})}{b^{*}(\tau, \vec{q})}=\tau+ \\
& +\frac{1}{2 \pi i m} \int d^{3} q \frac{e^{-\frac{4 \pi}{m^{2}} q^{2}}}{q^{2} \omega(q)} \ln \left[\frac{\omega(q) \int d^{3} \sigma e^{i \vec{q} \cdot \vec{\sigma}} \phi^{*}(\tau, \vec{\sigma})+i \int d^{3} \sigma e^{i \vec{q} \cdot \vec{\sigma}} \pi(\tau, \vec{\sigma})}{\omega(q) \int d^{3} \sigma e^{-i \vec{q} \cdot \vec{\sigma}} \phi(\tau, \vec{\sigma})-i \int d^{3} \sigma e^{-i \vec{q} \cdot \vec{\sigma}} \pi_{\phi^{*}}(\tau, \vec{\sigma})}\right]= \\
& \stackrel{\text { def }}{=} \tau+\tilde{X}_{b \phi}^{\tau}, \\
& \vec{X}_{b \phi}=\int d \tilde{q} \vec{q} F(q) \varphi_{b}(\tau, \vec{q})=\frac{1}{2 i} \int d \tilde{q} \vec{q} F(q) \ln \frac{b(\tau, \vec{q})}{b^{*}(\tau, \vec{q})}=
\end{aligned}
$$




$$
=\frac{2 i}{\pi m} \int d^{3} q \frac{\vec{q}}{q^{4}} e^{-\frac{4 \pi}{m^{2}} q^{2}} \ln \left[\frac{\sqrt{m^{2}+q^{2}} \int d^{3} \sigma e^{i \vec{q} \cdot \vec{\sigma}} \phi^{*}(\tau, \vec{\sigma})+i \int d^{3} \sigma e^{i \vec{q} \cdot \vec{\sigma}} \pi(\tau, \vec{\sigma})}{\sqrt{m^{2}+q^{2}} \int d^{3} \sigma e^{-i \vec{q} \cdot \vec{\sigma}} \phi(\tau, \vec{\sigma})-i \int d^{3} \sigma e^{-i \vec{q} \cdot \vec{\sigma}} \pi_{\phi^{*}}(\tau, \vec{\sigma})}\right] .
$$

Therefore, we shall define the canonical transformation of Section IV separately to $a, a^{*} \mapsto P_{a \phi}^{A}, X_{a \phi}^{A}\left(X_{a \phi}^{\tau}=\tau+\tilde{X}_{a \phi}^{\tau}\right), \mathbf{H}_{a}, \mathbf{K}_{a}$ and to $b, b^{*} \mapsto P_{b \phi}^{A}, X_{b \phi}^{A}\left(X_{b \phi}^{\tau}=\tau+\tilde{X}_{b \phi}\right)$, $\mathbf{H}_{b}, \mathbf{K}_{b}$ and not to $a_{i}, a_{i}^{*}, \mathrm{i}=1,2, \mapsto P_{i \phi}^{A}, X_{i \phi}^{A}, \mathbf{H}_{i}, \mathbf{K}_{i}$ [assuming that the fields $\phi_{a}, \pi_{a}, \phi_{b}, \pi_{b}$ satisfy Eq.(4.29)]. Then, we shall do the further canonical transformation from the two sets of collective variables $X_{a \phi}^{A}, P_{a \phi}^{A}, X_{b \phi}^{A}, P_{b \phi}^{A}$, to global center of phase variables $X_{\phi}^{A}, P_{\phi}^{A}$, and global relative variables $R_{\phi}^{A}, Q_{\phi}^{A}\left[R_{\phi}^{A}\right.$ describes the action-reaction between the centers of phase of positive and negative energy field configutations]

$$
\begin{aligned}
X_{\phi}^{A} & =\frac{1}{2}\left(X_{a \phi}^{A}+X_{b \phi}^{A}\right)\left[=\frac{1}{2}\left(X_{1 \phi}^{A}+X_{2 \phi}^{A}\right)\right], \quad X_{\phi}^{\tau}=\tau+\tilde{X}_{\phi}^{\tau}, \\
P_{\phi}^{A} & =P_{a \phi}^{A}+P_{b \phi}^{A}\left[=P_{1 \phi}^{A}+P_{2 \phi}^{A}\right], \\
R_{\phi}^{A} & =X_{a \phi}^{A}-X_{b \phi}^{A}, \\
Q_{\phi}^{A} & =\frac{1}{2}\left(P_{a \phi}^{A}-P_{b \phi}^{A}\right) .
\end{aligned}
$$

The Poincaré generators are $P_{\phi}^{\tau}, \vec{P}_{\phi}$ and

$$
\begin{aligned}
J_{\phi}^{r s} & =\int d^{3} \sigma\left[\pi_{\phi^{*}}\left(\sigma^{r} \partial^{s}-\sigma^{s} \partial^{r}\right) \phi^{*}+\pi_{\phi}\left(\sigma^{r} \partial^{s}-\sigma^{s} \partial^{r}\right) \phi\right](\tau, \vec{\sigma})= \\
& =-i \sum_{i=1}^{2} \int d \tilde{q} a_{i}^{*}(\tau, \vec{q})\left(q^{r} \frac{\partial}{\partial q^{s}}-q^{s} \frac{\partial}{\partial q^{r}}\right) a_{i}(\tau, \vec{q})= \\
& =-i \int d \tilde{q}\left[a^{*}(\tau, \vec{q})\left(q^{r} \frac{\partial}{\partial q^{s}}-q^{s} \frac{\partial}{\partial q^{r}}\right) a(\tau, \vec{q})+b^{*}(\tau, \vec{q})\left(q^{r} \frac{\partial}{\partial q^{s}}-q^{s} \frac{\partial}{\partial q^{r}}\right) b(\tau, \vec{q})\right], \\
J_{\phi}^{\tau r} & =-\tau P_{\phi}^{r}+\frac{1}{2} \int d^{3} \sigma \sigma^{r}\left[\pi_{\phi^{*}} \pi_{\phi}+\vec{\partial} \phi^{*} \cdot \vec{\partial} \phi+m^{2} \phi^{*} \phi\right](\tau, \vec{\sigma})= \\
& =-\tau P_{\phi}^{r}+i \sum_{i=1}^{2} \int d \tilde{q} \omega(q) a_{i}^{*}(\tau, \vec{q}) \frac{\partial}{\partial q^{r}} a_{i}(\tau, \vec{q})= \\
& =-\tau P_{\phi}^{r}+i \int d \tilde{q} \omega(q)\left[a^{*}(\tau, \vec{q}) \frac{\partial}{\partial q^{r}} a(\tau, \vec{q})+b^{*}(\tau, \vec{q}) \frac{\partial}{\partial q^{r}} b(\tau, \vec{q}) .\right.
\end{aligned}
$$

To find the real center of mass $\vec{q}_{\phi}$ we must use Eq.(6.1) of Section VI, namely $q_{\phi}^{r} \approx r_{\phi}^{r}=$ $-J_{\phi}^{\tau r} /\left.P_{\phi}^{\tau}\right|_{\tau=0}$.

The original variables have the following expression

$$
\begin{aligned}
a(\tau, \vec{k})= & \sqrt{F^{\tau}(k) \omega(k) P_{a \phi}^{\tau}-F(k) \vec{k} \cdot \vec{P}_{a \phi}(\tau)+\mathcal{D}_{k} \mathbf{H}_{a}(\tau, \vec{k})} \\
& e^{i \int d \tilde{q} \int d \tilde{q}^{\prime} \mathbf{K}_{a}(\tau, \vec{q}) \mathcal{G}\left(\vec{q}, \vec{q}^{\prime}\right) \Delta\left(\vec{q}^{\prime}, \vec{k}\right)+i \omega(k) X_{a \phi}^{\tau}(\tau)-i \vec{k} \cdot \vec{X}_{a \phi}(\tau)}, \\
b(\tau, \vec{q})= & \sqrt{F^{\tau}(k) \omega(k) P_{b \phi}^{\tau}(\tau)-F(k) \vec{k} \cdot \vec{P}_{b \phi}(\tau)+\mathcal{D}_{k} \mathbf{H}_{b}(\tau, \vec{k})} \\
& e^{i \int d \tilde{q} \int d \tilde{q}^{\prime} \mathbf{K}_{b}(\tau, \vec{q}) \mathcal{G}\left(\vec{q}, \vec{q}^{\prime}\right) \Delta\left(\vec{q}^{\prime}, \vec{k}\right)+i \omega(k) X_{b \phi}^{\tau}(\tau)-i \vec{k} \cdot \vec{X}_{b \phi}(\tau)},
\end{aligned}
$$




$$
\begin{aligned}
& \phi(\tau, \sigma)=\int d \tilde{k}\left(\sqrt{F^{\tau}(k) \omega(k) P_{a \phi}^{\tau}(\tau)-F(k) \vec{k} \cdot \vec{P}_{a \phi}(\tau)+\mathcal{D}_{k} \mathbf{H}_{a}(\tau, \vec{k})}\right. \\
& e^{i \int d \tilde{q} \int d \tilde{q}^{\prime} \mathbf{K}_{a}(\tau, \vec{q}) \mathcal{G}\left(\vec{q}, \vec{q}^{\prime}\right) \Delta\left(\vec{q}^{\prime}, \vec{k}\right)+i \omega(k) \tilde{X}_{a \phi}^{\tau}(\tau)+i \vec{k} \cdot\left(\vec{\sigma}-\vec{X}_{a \phi}(\tau)\right)}+ \\
& +\sqrt{F^{\tau}(k) \omega(k) P_{b \phi}^{\tau}(\tau)-F(k) \vec{k} \cdot \vec{P}_{b \phi}(\tau)+\mathcal{D}_{k} \mathbf{H}_{b}(\tau, \vec{k})} \\
& \left.e^{-i \int d \tilde{q} \int d \tilde{q}^{\prime} \mathbf{K}_{b}(\tau, \vec{q}) \mathcal{G}\left(\vec{q}, \vec{q}^{\prime}\right) \Delta\left(\vec{q}^{\prime}, \vec{k}\right)-i \omega(k) \tilde{X}_{b \phi}^{\tau}(\tau)-i \vec{k} \cdot\left(\vec{\sigma}-\vec{X}_{b \phi}(\tau)\right)}\right)= \\
& =\int d \tilde{k}\left(\sqrt{F^{\tau}(k) \omega(k)\left[\frac{1}{2} P_{\phi}^{\tau}+Q_{\phi}^{\tau}(\tau)\right]-F(k) \vec{k} \cdot\left[\frac{1}{2} \vec{P}_{\phi}+\vec{Q}_{\phi}(\tau)\right]+\mathcal{D}_{k} \mathbf{H}_{a}(\tau, \vec{k})}\right.
\end{aligned}
$$

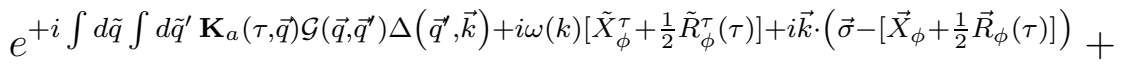

$$
\begin{aligned}
& +\sqrt{F^{\tau}(k) \omega(k)\left[\frac{1}{2} P_{\phi}^{\tau}-Q_{\phi}^{\tau}(\tau)\right]-F(k) \vec{k} \cdot\left[\frac{1}{2} \vec{P}_{\phi}-\vec{Q}_{\phi}(\tau)\right]+\mathcal{D}_{k} \mathbf{H}_{b}(\tau, \vec{k})} \\
& \left.e^{-i \int d \tilde{q} \int d \tilde{q}^{\prime} \mathbf{K}_{b}(\tau, \vec{q}) \mathcal{G}\left(\vec{q}, \vec{q}^{\prime}\right) \Delta\left(\vec{q}^{\prime}, \vec{k}\right)-i \omega(k)\left[\tilde{X}_{\phi}^{\tau}-\frac{1}{2} \tilde{R}_{\phi}^{\tau}(\tau)\right]-i \vec{k} \cdot\left(\vec{\sigma}-\left[\vec{X}_{\phi}-\frac{1}{2} \vec{R}_{\phi}(\tau)\right]\right)}\right)= \\
& =\int d \tilde{k}\left[\mathbf{A}_{a \vec{k}}\left(\tau ; \frac{1}{2} P_{\phi}^{A}+Q_{\phi}^{A}, \mathbf{H}_{a}\right] e^{i\left(\vec{k} \cdot \vec{\sigma}+\mathbf{B}_{a \vec{k}}\left(\tau ; X_{\phi}^{A}+\frac{1}{2} R_{\phi}^{A}, \mathbf{K}_{a}\right]\right)}+\right. \\
& \left.+\mathbf{A}_{b \vec{k}}\left(\tau ; \frac{1}{2} P_{\phi}^{A}-Q_{\phi}^{A}, \mathbf{H}_{b}\right] e^{-i\left(\vec{k} \cdot \vec{\sigma}+\mathbf{B}_{b \vec{k}}\left(\tau ; X_{\phi}^{A}-\frac{1}{2} R_{\phi}^{A}, \mathbf{K}_{b}\right]\right)}\right], \\
& \pi(\tau, \vec{\sigma})=-i \int d \tilde{k} \omega(k) \\
& \left(\sqrt{F^{\tau}(k) \omega(k)\left[\frac{1}{2} P_{\phi}^{\tau}-Q_{\phi}^{\tau}(\tau)\right]-F(k) \vec{k} \cdot\left[\frac{1}{2} \vec{P}_{\phi}-\vec{Q}_{\phi}(\tau)\right]+\mathcal{D}_{k} \mathbf{H}_{b}(\tau, \vec{k})}\right. \\
& e^{+i \int d \tilde{q} \int d \tilde{q}^{\prime} \mathbf{K}_{b}(\tau, \vec{q}) \mathcal{G}\left(\vec{q}, \vec{q}^{\prime}\right) \Delta\left(\vec{q}^{\prime}, \vec{k}\right)+i \omega(k)\left[\tilde{X}_{\phi}^{\tau}-\frac{1}{2} \tilde{R}_{\phi}^{\tau}(\tau)\right]+i \vec{k} \cdot\left(\vec{\sigma}-\left[\vec{X}_{\phi}-\frac{1}{2} \vec{R}_{\phi}(\tau)\right]\right)} \\
& -\sqrt{F^{\tau}(k) \omega(k)\left[\frac{1}{2} P_{\phi}^{\tau}+Q_{\phi}^{\tau}(\tau)\right]-F(k) \vec{k} \cdot\left[\frac{1}{2} \vec{P}_{\phi}+\vec{Q}_{\phi}(\tau)\right]+\mathcal{D}_{k} \mathbf{H}_{a}(\tau, \vec{k})} \\
& \left.e^{-i \int d \tilde{q} \int d \tilde{q}^{\prime} \mathbf{K}_{a}(\tau, \vec{q}) \mathcal{G}\left(\vec{q}, \vec{q}^{\prime}\right) \Delta\left(\vec{q}^{\prime}, \vec{k}\right)-i \omega(k)\left[\tilde{X}_{\phi}^{\tau}+\frac{1}{2} \tilde{R}_{\phi}^{\tau}(\tau)\right]-i \vec{k} \cdot\left(\vec{\sigma}-\left[\vec{X}_{\phi}+\frac{1}{2} \vec{R}_{\phi}(\tau)\right]\right)}\right)= \\
& =i \int d \tilde{k} \omega(k) \\
& {\left[\mathbf{A}_{a \vec{k}}\left(\tau ; \frac{1}{2} P_{\phi}^{A}+Q_{\phi}^{A}, \mathbf{H}_{a}\right] e^{-i\left(\vec{k} \cdot \vec{\sigma}+\mathbf{B}_{a \vec{k}}\left(\tau ; X_{\phi}^{A}+\frac{1}{2} R_{\phi}^{A}, \mathbf{K}_{a}\right]\right)}-\right.} \\
& \left.-\mathbf{A}_{b \vec{k}}\left(\tau ; \frac{1}{2} P_{\phi}^{A}-Q_{\phi}^{A}, \mathbf{H}_{b}\right] e^{i\left(\vec{k} \cdot \vec{\sigma}+\mathbf{B}_{b \vec{k}}\left(\tau ; X_{\phi}^{A}-\frac{1}{2} R_{\phi}^{A}, \mathbf{K}_{b}\right]\right)}\right],
\end{aligned}
$$

$$
\begin{aligned}
& \mathbf{A}_{a \vec{k}}\left(\tau ; \frac{1}{2} P_{\phi}^{A}+Q_{\phi}^{A}, \mathbf{H}_{a}\right]= \\
& \sqrt{F^{\tau}(k) \omega(k)\left[\frac{1}{2} P_{\phi}^{\tau}+Q_{\phi}^{\tau}(\tau)\right]-F(k) \vec{k} \cdot\left[\frac{1}{2} \vec{P}_{\phi}+\vec{Q}_{\phi}(\tau)\right]+\mathcal{D}_{\vec{k}} \mathbf{H}_{a}(\tau, \vec{k})}, \\
& \mathbf{A}_{b \vec{k}}\left(\tau ; \frac{1}{2} P_{\phi}^{A}-Q_{\phi}^{A}, \mathbf{H}_{b}\right]= \\
& \sqrt{F^{\tau}(k) \omega(k)\left[\frac{1}{2} P_{\phi}^{\tau}-Q_{\phi}^{\tau}(\tau)\right]-F(k) \vec{k} \cdot\left[\frac{1}{2} \vec{P}_{\phi}-\vec{Q}_{\phi}(\tau)\right]+\mathcal{D}_{\vec{k}} \mathbf{H}_{b}(\tau, \vec{k})},
\end{aligned}
$$




$$
\begin{aligned}
& \mathbf{B}_{a \vec{k}}\left(\tau ; X_{\phi}^{A}+\frac{1}{2} R_{\phi}^{A}, \mathbf{K}_{a}\right]=i \omega(k)\left[\tilde{X}_{\phi}^{\tau}+\frac{1}{2} R_{\phi}^{\tau}(\tau)\right]-i \vec{k} \cdot\left[\vec{X}_{\phi}+\frac{1}{2} \vec{R}_{\phi}(\tau)\right]+ \\
+ & \int d \tilde{q} d \tilde{q}^{\prime} \mathbf{K}_{a}(\tau, \vec{q}) \mathcal{G}\left(\vec{q}, \vec{q}^{\prime}\right) \triangle(\vec{q}, \vec{k}), \\
& \mathbf{B}_{b \vec{k}}\left(\tau ; X_{\phi}^{A}-\frac{1}{2} R_{\phi}^{A}, \mathbf{K}_{a}\right]=i \omega(k)\left[\tilde{X}_{\phi}^{\tau}-\frac{1}{2} R_{\phi}^{\tau}(\tau)\right]-i \vec{k} \cdot\left[\vec{X}_{\phi}-\frac{1}{2} \vec{R}_{\phi}(\tau)\right]+ \\
+ & \int d \tilde{q} d \tilde{q}^{\prime} \mathbf{K}_{b}(\tau, \vec{q}) \mathcal{G}(\vec{q}, \vec{q}) \triangle(\vec{q}, \vec{k}),
\end{aligned}
$$

In the gauge $\chi=T_{s}-\tau \approx 0$ we get

$$
\begin{aligned}
& \phi\left(T_{s}, \vec{\sigma}\right)=\int d \tilde{k}\left(\sqrt{F^{\tau}(k) \omega(k)\left[\frac{1}{2} P_{\phi}^{\tau}+Q_{\phi}^{\tau}\left(T_{s}\right)\right]-F(k) \vec{k} \cdot \vec{Q}_{\phi}\left(T_{s}\right)+\mathcal{D}_{k} \mathbf{H}_{a}\left(T_{s}, \vec{k}\right)}\right. \\
& e^{i \int d \tilde{q} \int d \tilde{q}^{\prime} \mathbf{K}_{a}\left(T_{s}, \vec{q}\right) \mathcal{G}\left(\vec{q}, \vec{q}^{\prime}\right) \Delta\left(\vec{q}^{\prime}, \vec{k}\right)+i \omega(k)\left[\tilde{X}_{\phi}^{\tau}+\frac{1}{2} \tilde{R}_{\phi}^{\tau}\left(T_{s}\right)\right]+i \vec{k} \cdot\left(\vec{\sigma}-\frac{1}{2} \vec{R}_{\phi}\left(T_{s}\right)\right)}+ \\
& +\sqrt{F^{\tau}(k) \omega(k)\left[\frac{1}{2} P_{\phi}^{\tau}-Q_{\phi}^{\tau}\left(T_{s}\right)\right]+F(k) \vec{k} \cdot \vec{Q}_{\phi}\left(T_{s}\right)+\mathcal{D}_{k} \mathbf{H}_{b}\left(T_{s}, \vec{k}\right)} \\
& \left.e^{-i \int d \tilde{q} \int d \tilde{q}^{\prime} \mathbf{K}_{b}\left(T_{s}, \vec{q}\right) \mathcal{G}\left(\vec{q}, \vec{q}^{\prime}\right) \Delta\left(\vec{q}^{\prime}, \vec{k}\right)-i \omega(k)\left[\tilde{X}_{\phi}^{\tau}-\frac{1}{2} \tilde{R}_{\phi}^{\tau}\left(T_{s}\right)\right]-i \vec{k} \cdot\left(\vec{\sigma}+\frac{1}{2} \vec{R}_{\phi}\left(T_{s}\right)\right)}\right), \\
& \pi\left(T_{s}, \vec{\sigma}\right)=-i \int d \tilde{k} \omega(k) \\
& \left(\sqrt{F^{\tau}(k) \omega(k)\left[\frac{1}{2} P_{\phi}^{\tau}-Q_{\phi}^{\tau}\left(T_{s}\right)\right]+F(k) \vec{k} \cdot \vec{Q}_{\phi}\left(T_{s}\right)+\mathcal{D}_{k} \mathbf{H}_{b}\left(T_{s}, \vec{k}\right)}\right. \\
& e^{+i \int d \tilde{q} \int d \tilde{q}^{\prime} \mathbf{K}_{b}\left(T_{s}, \vec{q}\right) \mathcal{G}\left(\vec{q}, \vec{q}^{\prime}\right) \Delta\left(\vec{q}^{\prime}, \vec{k}\right)+i \omega(k)\left[\tilde{X}_{\phi}^{\tau}-\frac{1}{2} \tilde{R}_{\phi}^{\tau}\left(T_{s}\right)\right]+i \vec{k} \cdot\left(\vec{\sigma}+\frac{1}{2} \vec{R}_{\phi}\left(T_{s}\right)\right)} \\
& -\sqrt{F^{\tau}(k) \omega(k)\left[\frac{1}{2} P_{\phi}^{\tau}+Q_{\phi}^{\tau}\left(T_{s}\right)\right]-F(k) \vec{k} \cdot \vec{Q}_{\phi}\left(T_{s}\right)+\mathcal{D}_{k} \mathbf{H}_{a}\left(T_{s}, \vec{k}\right)} \\
& \left.e^{-i \int d \tilde{q} \int d \tilde{q}^{\prime} \mathbf{K}_{a}\left(T_{s}, \vec{q}\right) \mathcal{G}\left(\vec{q}, \vec{q}^{\prime}\right) \Delta\left(\vec{q}^{\prime}, \vec{k}\right)-i \omega(k)\left[\tilde{X}_{\phi}^{\tau}+\frac{1}{2} \tilde{R}_{\phi}^{\tau}\left(T_{s}\right)\right]-i \vec{k} \cdot\left(\vec{\sigma}-\frac{1}{2} \vec{R}_{\phi}\left(T_{s}\right)\right)}\right), \\
& N_{\phi}=P_{\phi}^{\tau} \int d \tilde{k} \omega(k) F^{\tau}(k)+\int d \tilde{k}\left(-F(k) \vec{k} \cdot \vec{P}_{\phi}+\mathcal{D}_{k}\left[\mathbf{H}_{a}\left(T_{s}, \vec{k}\right)+\mathbf{H}_{b}\left(T_{s}, \vec{k}\right)\right]\right) \approx \\
& \approx P_{\phi}^{\tau} \int d \tilde{k} \omega(k) F^{\tau}(k)+\int d \tilde{k} \mathcal{D}_{k}\left[\mathbf{H}_{a}\left(T_{s}, \vec{k}\right)+\mathbf{H}_{b}\left(T_{s}, \vec{k}\right)\right] \\
& q_{\phi}=2 Q_{\phi}^{\tau}\left(T_{s}\right) \int d \tilde{k} \omega(k) F^{\tau}(k)+\int d \tilde{k}\left(-2 F(k) \vec{k} \cdot \vec{Q}_{\phi}\left(T_{s}\right)+\mathcal{D}_{k}\left[\mathbf{H}_{a}\left(T_{s}, \vec{k}\right)-\mathbf{H}_{b}\left(T_{s}, \vec{k}\right)\right]\right) .
\end{aligned}
$$

\section{The coupling to the electromagnetic field.}

Let us consider the action describing a charged Klein Gordon field interacting with the electromagnetic field on spacelike hypersurfaces following the scheme of Ref. [8]

$$
\begin{aligned}
S= & \int d \tau d^{3} \sigma N(\tau, \vec{\sigma}) \sqrt{\gamma(\tau, \vec{\sigma})} \\
& \left\{g^{\tau \tau}\left(\partial_{\tau}+i e A_{\tau}\right) \phi^{*}\left(\partial_{\tau}-i e A_{\tau}\right) \phi+\right. \\
& +g^{\tau \check{r}}\left[\left(\partial_{\tau}+i e A_{\tau}\right) \phi^{*}\left(\partial_{\check{r}}-i e A_{\check{r}}\right) \phi+\left(\partial_{\check{r}}+i e A_{\check{r}}\right) \phi^{*}\left(\partial_{\tau}-i e A_{\tau}\right) \phi\right]+
\end{aligned}
$$




$$
\begin{aligned}
& \left.+g^{\check{r} \check{s}}\left(\partial_{\check{r}}+i e A_{\check{r}}\right) \phi^{*}\left(\partial_{\check{s}}-i e A_{\check{s}}\right) \phi-m^{2} \phi^{*} \phi-\frac{1}{4} g^{\check{A} \check{C}} g^{\check{B} \check{D}} F_{\check{A} \check{B}} F_{\check{C} \check{D}}\right\}(\tau, \vec{\sigma})= \\
& =\int d \tau d^{3} \sigma \sqrt{\gamma(\tau, \vec{\sigma})}\left\{\frac{1}{N}\left[\partial_{\tau}+i e A_{\tau}-N^{\check{r}}\left(\partial_{\check{r}}+i e A_{\check{r}}\right)\right] \phi^{*}\right. \\
& {\left[\partial_{\tau}-i e A_{\tau}-N^{\check{s}}\left(\partial_{\check{s}}-i e A_{\check{s}}\right)\right] \phi+N\left[\gamma^{\check{r} \check{s}}\left(\partial_{\check{r}}+i e A_{\check{r}}\right) \phi^{*}\left(\partial_{\check{s}}-i e A_{\check{s}}\right) \phi-m^{2} \phi^{*} \phi\right]-} \\
& \left.-\frac{1}{2 N}\left(F_{\tau \check{r}}-N^{\check{u}} F_{\check{u} \check{r}}\right) \gamma^{\check{r} \check{s}}\left(F_{\tau \check{s}}-N^{\check{v}} F_{\check{v} \check{s}}\right)-\frac{N}{4} \gamma^{\check{r} \check{s}} \gamma^{\check{u} \check{v}} F_{\check{r} \check{u}} F_{\check{s} \check{v}}\right\}(\tau, \vec{\sigma}) .
\end{aligned}
$$

where the configuration variables are $z^{\mu}(\tau, \vec{\sigma}), \phi(\tau, \vec{\sigma})=\tilde{\phi}(z(\tau, \vec{\sigma}))$ and $A_{\check{A}}(\tau, \vec{\sigma})=$ $z_{\tilde{A}}^{\mu}(\tau, \vec{\sigma}) \tilde{A}_{\mu}(z(\tau, \vec{\sigma}))\left[\tilde{\phi}(z)\right.$ and $\tilde{A}_{\mu}(z)$ are the standard Klein-Gordon field and electromagnetic potential, which do not know the embedding of the spacelike hypersurface $\Sigma$ in Minkowski spacetime like $\phi$ and $\left.A_{\check{A}}\right]$.

The canonical momenta are

$$
\begin{aligned}
& \pi^{\tau}(\tau, \vec{\sigma})=\frac{\partial L}{\partial \partial_{\tau} A_{\tau}(\tau, \vec{\sigma})}=0, \\
& \pi^{\check{r}}(\tau, \vec{\sigma})=\frac{\partial L}{\partial \partial_{\tau} A_{\check{r}}(\tau, \vec{\sigma})}=-\frac{\sqrt{\gamma(\tau, \vec{\sigma})}}{N(\tau, \vec{\sigma})} \gamma^{\check{r} \check{s}}(\tau, \vec{\sigma})\left(F_{\tau \check{s}}-N^{\check{u}} F_{\breve{u} \check{s}}\right)(\tau, \vec{\sigma}), \\
& \pi_{\phi}(\tau, \vec{\sigma})=\frac{\partial L}{\partial \partial_{\tau} \phi(\tau, \vec{\sigma})}=\frac{\sqrt{\gamma(\tau, \vec{\sigma})}}{N(\tau, \vec{\sigma})}\left[\partial_{\tau}+i e A_{\tau}-N^{\check{r}}\left(\partial_{\check{r}}+i e A_{\check{r}}\right)\right](\tau, \vec{\sigma}) \phi^{*}(\tau, \vec{\sigma}), \\
& \pi_{\phi^{*}}(\tau, \vec{\sigma})=\frac{\partial L}{\partial \partial_{\tau} \phi^{*}(\tau, \vec{\sigma})}=\frac{\sqrt{\gamma(\tau, \vec{\sigma})}}{N(\tau, \vec{\sigma})}\left[\partial_{\tau}-i e A_{\tau}-N^{\check{r}}\left(\partial_{\check{r}}-i e A_{\check{r}}\right)\right](\tau, \vec{\sigma}) \phi(\tau, \vec{\sigma}), \\
& \rho_{\mu}(\tau, \vec{\sigma})=-\frac{\partial L}{\partial \partial_{\tau} z^{\mu}(\tau, \vec{\sigma})}= \\
& =l_{\mu}(\tau, \vec{\sigma})\left\{\frac{\pi_{\phi} \pi_{\phi^{*}}}{\sqrt{\gamma}}-\sqrt{\gamma}\left[\gamma^{\check{r} \check{s}}\left(\partial_{\check{r}}+i e A_{\check{r}}\right) \phi^{*}\left(\partial_{\check{s}}-i e A_{\check{s}}\right) \phi-\right.\right. \\
& \left.\left.-m^{2} \phi^{*} \phi\right]+\frac{1}{2 \sqrt{\gamma}} \pi^{\check{r}} g_{\check{r} \check{s}} \pi^{\check{s}}-\frac{\sqrt{\gamma}}{4} \gamma^{\check{r} \check{s}} \gamma^{\check{u} \check{v}} F_{\check{r} \check{u}} F_{\check{s} \check{v}}\right\}(\tau, \vec{\sigma})+ \\
& +z_{\check{s} \mu}(\tau, \vec{\sigma}) \gamma^{\check{r} \check{s}}(\tau, \vec{\sigma})\left\{\pi_{\phi^{*}}\left(\partial_{\check{r}}+i e A_{\check{r}}\right) \phi^{*}+\pi_{\phi}\left(\partial_{\check{r}}-i e A_{\check{r}}\right) \phi-F_{\check{r} \check{u}} \pi^{\check{u}}\right\}(\tau, \vec{\sigma}) .
\end{aligned}
$$

Therefore, we have the following primary constraints

$$
\begin{aligned}
\pi^{\tau}(\tau, \vec{\sigma}) \approx & 0, \\
\mathcal{H}_{\mu}(\tau, \vec{\sigma})= & \rho_{\mu}(\tau, \vec{\sigma})- \\
& -l_{\mu}(\tau, \vec{\sigma})\left\{\frac{\pi_{\phi} \pi_{\phi^{*}}}{\sqrt{\gamma}}-\sqrt{\gamma}\left[\gamma^{\check{r} \check{s}}\left(\partial_{\check{r}}+i e A_{\check{r}}\right) \phi^{*}\left(\partial_{\check{s}}-i e A_{\breve{s}}\right) \phi-\right.\right. \\
& \left.\left.-m^{2} \phi^{*} \phi\right]+\frac{1}{2 \sqrt{\gamma}} \pi^{\check{r}} g_{\check{r} \check{s} \check{s}} \pi^{\check{s}}-\frac{\sqrt{\gamma}}{4} \gamma^{\check{r} \check{s}} \gamma^{\breve{u} \check{v}} F_{\check{r} \check{u}} F_{\check{s} \check{v}}\right\}(\tau, \vec{\sigma})+ \\
& +z_{\check{s} \mu}(\tau, \vec{\sigma}) \gamma^{\check{r} \check{s}}(\tau, \vec{\sigma})\left\{\pi_{\phi^{*}}\left(\partial_{\check{r}}+i e A_{\check{r}}\right) \phi^{*}+\pi_{\phi}\left(\partial_{\check{r}}-i e A_{\check{r}}\right) \phi-F_{\check{r} \check{u}} \pi^{\check{u}}\right\}(\tau, \vec{\sigma}) \approx 0,
\end{aligned}
$$

and the following Dirac Hamiltonian $\left[\lambda(\tau, \vec{\sigma})\right.$ and $\lambda^{\mu}(\tau, \vec{\sigma})$ are Dirac multiplier $]$

$$
H_{D}=\int d^{3} \sigma\left[-A_{\tau}(\tau, \vec{\sigma}) \Gamma(\tau, \vec{\sigma})+\lambda(\tau, \vec{\sigma}) \pi^{\tau}(\tau, \vec{\sigma})+\lambda^{\mu}(\tau, \vec{\sigma}) \mathcal{H}_{\mu}(\tau, \vec{\sigma})\right] .
$$


By using the Poisson brackets

$$
\begin{aligned}
\left\{z^{\mu}(\tau, \vec{\sigma}), \rho_{\nu}\left(\tau, \vec{\sigma}^{\prime}\right)\right\} & =\eta_{\nu}^{\mu} \delta^{3}\left(\vec{\sigma}-\vec{\sigma}^{\prime}\right), \\
\left\{A_{\check{A}}(\tau, \vec{\sigma}), \pi^{\check{B}}\left(\tau, \vec{\sigma}^{\prime}\right)\right\} & =\eta_{\check{A}}^{\breve{B}} \delta^{3}\left(\vec{\sigma}-\vec{\sigma}^{\prime}\right), \\
\left\{\phi(\tau, \vec{\sigma}), \pi_{\phi}\left(\tau, \vec{\sigma}^{\prime}\right)\right\} & =\left\{\phi^{*}(\tau, \vec{\sigma}), \pi_{\phi^{*}}\left(\tau, \vec{\sigma}^{\prime}\right)\right\}=\delta^{3}\left(\vec{\sigma}-\vec{\sigma}^{\prime}\right),
\end{aligned}
$$

we find that the time constancy of the primary constraints implies the existence of only one secondary constraint

$$
\Gamma(\tau, \vec{\sigma})=\partial_{\check{r}} \pi^{\check{r}}(\tau, \vec{\sigma})+i e\left(\pi_{\phi^{*}} \phi^{*}-\pi_{\phi} \phi\right)(\tau, \vec{\sigma}) \approx 0
$$

We can verify that these constraints are first class with the algebra given in Eqs.(125) of Ref. [1].

The Poincaré generators are like in Eq.(2.6).

On spacelike hyperplanes $z^{\mu}(\tau, \vec{\sigma})=x_{s}^{\mu}(\tau)+b_{\check{r}}^{\mu}(\tau) \sigma^{\check{r}}$, the constraints are reduced to the following ones

$$
\begin{aligned}
\pi^{\tau}(\tau, \vec{\sigma}) & \approx 0 \\
\Gamma(\tau, \vec{\sigma}) & =-\vec{\partial} \vec{\pi}(\tau, \vec{\sigma})+i e\left[\pi_{\phi^{*}} \phi^{*}-\pi_{\phi} \phi\right](\tau, \vec{\sigma}) \approx 0 \\
\tilde{\mathcal{H}}^{\mu}(\tau) & =\int d^{3} \sigma \mathcal{H}^{\mu}(\tau, \vec{\sigma})= \\
& =p_{s}^{\mu}-l^{\mu}\left\{\frac{1}{2} \int d^{3} \sigma\left[\vec{\pi}^{2}+\vec{B}^{2}\right](\tau, \vec{\sigma})+\right. \\
& \left.+\int d^{3} \sigma\left[\pi_{\phi^{*}} \pi_{\phi}+(\vec{\partial}+i e \vec{A}) \phi^{*} \cdot(\vec{\partial}-i e \vec{A}) \phi+m^{2} \phi^{*} \phi\right](\tau, \vec{\sigma})\right\}- \\
& -b_{\check{r}}^{\mu}(\tau)\left\{\int d^{3} \sigma(\vec{\pi} \times \vec{B})_{\check{r}}(\tau, \vec{\sigma})+\int d^{3} \sigma\left[\pi_{\phi^{*}}\left(\partial_{\check{r}}+i e A_{\check{r}}\right) \phi^{*}+\right.\right. \\
& \left.\left.+\pi_{\phi}\left(\partial_{\check{r}}-i e A_{\check{r}}\right) \phi\right](\tau, \vec{\sigma})\right\} \approx 0 \\
\tilde{\mathcal{H}}^{\mu \nu}(\tau) & =b_{\check{r}}^{\mu}(\tau) \int d^{3} \sigma \sigma^{\check{r}} \mathcal{H}^{\nu}(\tau, \vec{\sigma})-b_{\check{r}}^{\nu}(\tau) \int d^{3} \sigma \sigma^{\check{r}} \mathcal{H}^{\mu}(\tau, \vec{\sigma})= \\
& =S_{s}^{\mu \nu}-\left(b_{\check{r}}^{\mu}(\tau) l^{\nu}-b_{\check{r}}^{\nu}(\tau) l^{\mu}\right)\left[\frac{1}{2} \int d^{3} \sigma \sigma^{\check{r}}\left(\vec{\pi}^{2}+\vec{B}^{2}\right)(\tau, \vec{\sigma})+\right. \\
& \left.+\int d^{3} \sigma \sigma^{\check{r}}\left[\pi_{\phi^{*}} \pi_{\phi}+(\vec{\partial}+i e \vec{A}) \phi^{*} \cdot(\vec{\partial}-i e \vec{A}) \phi+m^{2} \phi^{*} \phi\right](\tau, \vec{\sigma})\right\}+ \\
& +\left(b_{\check{r}}^{\mu}(\tau) b_{\check{s}}^{\nu}(\tau)-b_{\check{r}}^{\nu}(\tau) b_{\check{s}}^{\mu}(\tau)\right)\left\{\int d^{3} \sigma \sigma^{\check{r}}(\vec{\pi} \times \vec{B})_{\check{s}}(\tau, \vec{\sigma})+\right. \\
& \left.+\int d^{3} \sigma \sigma^{\check{r}}\left[\pi_{\phi^{*}}\left(\partial_{\check{s}}+i e A_{\check{s}}\right) \phi^{*}+\pi_{\phi}\left(\partial_{\check{s}}-i e A_{\check{s}}\right) \phi\right](\tau, \vec{\sigma})\right\} \approx 0
\end{aligned}
$$

With the final restriction to Wigner hyperplanes, where the canonical variables are $\tilde{x}_{s}^{\mu}(\tau), p_{s}^{\mu}, A_{\tau}(\tau, \vec{\sigma}), \pi^{\tau}(\tau, \vec{\sigma}), \vec{A}(\tau, \vec{\sigma}), \vec{\pi}(\tau, \vec{\sigma}), \phi(\tau, \vec{\sigma}), \pi_{\phi}(\tau, \vec{\sigma})=\left[\dot{\phi}^{*}-i e A_{\tau}\right](\tau, \vec{\sigma}), \phi^{*}(\tau, \vec{\sigma})$, $\pi_{\phi^{*}}(\tau, \vec{\sigma})=\left[\dot{\phi}-i e A_{\tau}\right](\tau, \vec{\sigma})$, the only surviving constraints are

$$
\begin{aligned}
\pi^{\tau}(\tau, \vec{\sigma}) & \approx 0, \\
\Gamma(\tau, \vec{\sigma}) & =-\vec{\partial} \vec{\pi}(\tau, \vec{\sigma})+i e\left[\pi_{\phi^{*}} \phi^{*}-\pi_{\phi} \phi\right](\tau, \vec{\sigma}) \approx 0, \\
\mathcal{H}(\tau) & =\epsilon_{s}-\left\{\frac{1}{2} \int d^{3} \sigma\left(\vec{\pi}^{2}+\vec{B}^{2}\right)(\tau, \vec{\sigma})+\right.
\end{aligned}
$$




$$
\begin{aligned}
& \left.+\int d^{3} \sigma\left[\pi_{\phi^{*}} \phi^{*} \pi_{\phi}+(\vec{\partial}+i e \vec{A}) \phi^{*} \cdot(\vec{\partial}-i e \vec{A}) \phi+m^{2} \phi^{*} \phi\right](\tau, \vec{\sigma})\right\} \approx 0, \\
\overrightarrow{\mathcal{H}}_{p}(\tau) & =\int d^{3} \sigma(\vec{\pi} \times \vec{B})(\tau, \vec{\sigma})+ \\
& +\int d^{3} \sigma\left[\pi_{\phi^{*}}(\vec{\partial}+i e \vec{A}) \phi^{*}+\pi_{\phi}(\vec{\partial}-i e \vec{A}) \phi\right](\tau, \vec{\sigma}) \approx 0 .
\end{aligned}
$$

In the Lorentz generators of Eq.(2.9) the spin tensor has the form

$$
\begin{aligned}
\bar{S}_{s}^{r s} & \equiv S_{\phi}^{r s}=\int d^{3} \sigma\left\{\sigma^{r}(\vec{\pi} \times \vec{B})^{s}(\tau, \vec{\sigma})-\sigma^{s}(\vec{\pi} \times \vec{B})^{r}(\tau, \vec{\sigma})\right\}+ \\
& +\int d^{3} \sigma\left\{\sigma^{r}\left[\pi_{\phi^{*}}\left(\partial^{s}+i e A^{s}\right) \phi^{*}+\pi_{\phi}\left(\partial^{s}-i e A^{s}\right) \phi\right](\tau, \vec{\sigma})-(r \leftrightarrow s)\right\} .
\end{aligned}
$$

To make the reduction to Dirac's observables with respect to the electromagnetic gauge transformations, let us recall [20,21] that the electromagnetic gauge degrees of freedom are described by the two pairs of conjugate variables $A_{\tau}(\tau, \vec{\sigma}), \pi_{\tau}(\tau, \vec{\sigma})[\approx 0], \eta_{e m}(\tau, \vec{\sigma})=$ $-\frac{1}{\Delta} \frac{\partial}{\partial \vec{\sigma}} \cdot \vec{A}(\tau, \vec{\sigma}), \Gamma(\tau, \vec{\sigma})[\approx 0]$, so that we have the decompositions

$$
\begin{aligned}
A^{r}(\tau, \vec{\sigma}) & =\frac{\partial}{\partial \sigma^{r}} \eta_{e m}(\tau, \vec{\sigma})+A_{\perp}^{r}(\tau, \vec{\sigma}), \\
\pi^{r}(\tau, \vec{\sigma}) & =\pi_{\perp}^{r}(\tau, \vec{\sigma})+ \\
& +\frac{1}{\triangle} \frac{\partial}{\partial \sigma^{r}}\left[-\Gamma(\tau, \vec{\sigma})+i e\left(\pi_{\phi^{*}} \phi^{*}-\pi_{\phi} \phi\right)(\tau, \vec{\sigma})\right] \approx 0, \\
\left\{A_{\perp}^{r}(\tau, \vec{\sigma}),\right. & \left.\pi_{\perp}^{s}\left(\tau, \vec{\sigma}^{\prime}\right)\right\}=-P_{\perp}^{r s}(\vec{\sigma}) \delta^{3}\left(\vec{\sigma}-\vec{\sigma}^{\prime}\right),
\end{aligned}
$$

where $P_{\perp}^{r s}(\vec{\sigma})=\delta^{r s}+\frac{\partial^{r} \partial^{s}}{\triangle}, \triangle=-\vec{\partial}^{2}$. Then, we have

$$
\begin{aligned}
\int d^{3} \sigma & \vec{\pi}^{2}(\tau, \vec{\sigma})=\int d^{3} \sigma \vec{\pi}_{\perp}^{2}(\tau, \vec{\sigma})- \\
- & \frac{e^{2}}{4 \pi} \int d^{3} \sigma_{1} d^{3} \sigma_{2} \frac{i\left(\pi_{\phi^{*}} \phi^{*}-\pi_{\phi} \phi\right)\left(\tau, \vec{\sigma}_{1}\right) i\left(\pi_{\phi^{*}} \phi^{*}-\pi_{\phi} \phi\right)\left(\tau, \vec{\sigma}_{2}\right)}{\left|\vec{\sigma}_{1}-\vec{\sigma}_{2}\right|} .
\end{aligned}
$$

Since we have

$$
\begin{aligned}
\left\{\phi(\tau, \vec{\sigma}), \Gamma\left(\tau, \vec{\sigma}^{\prime}\right)\right\} & =-i e \phi(\tau, \vec{\sigma}) \delta^{3}\left(\vec{\sigma}-\vec{\sigma}^{\prime}\right) \\
\left\{\phi^{*}(\tau, \vec{\sigma}), \Gamma\left(\tau, \vec{\sigma}^{\prime}\right)\right\} & =i e \phi^{*}(\tau, \vec{\sigma}) \delta^{3}\left(\vec{\sigma}-\vec{\sigma}^{\prime}\right) \\
\left\{\pi_{\phi}(\tau, \vec{\sigma}), \Gamma\left(\tau, \vec{\sigma}^{\prime}\right)\right\} & =i e \pi_{\phi}(\tau, \vec{\sigma}) \delta^{3}\left(\vec{\sigma}-\vec{\sigma}^{\prime}\right) \\
\left\{\pi_{\phi^{*}}(\tau, \vec{\sigma}), \Gamma\left(\tau, \vec{\sigma}^{\prime}\right)\right\} & =-i e \pi_{\phi^{*}}(\tau, \vec{\sigma}) \delta^{3}\left(\vec{\sigma}-\vec{\sigma}^{\prime}\right)
\end{aligned}
$$

the Dirac observables for the Klein-Gordon field are

$$
\begin{aligned}
\hat{\phi}(\tau, \vec{\sigma}) & =e^{-i e \eta_{e m}(\tau, \vec{\sigma})} \phi(\tau, \vec{\sigma}), \\
\hat{\phi}^{*}(\tau, \vec{\sigma}) & =e^{i e \eta_{e m}(\tau, \vec{\sigma})} \phi^{*}(\tau, \vec{\sigma}), \\
\hat{\pi}_{\phi}(\tau, \vec{\sigma}) & =e^{i e \eta_{e m}(\tau, \vec{\sigma})} \pi_{\phi}(\tau, \vec{\sigma}), \\
\hat{\pi}_{\phi^{*}}(\tau, \vec{\sigma}) & =e^{-i e \eta_{e m}(\tau, \vec{\sigma})} \pi_{\phi^{*}}(\tau, \vec{\sigma}),
\end{aligned}
$$




$$
\begin{array}{r}
\left\{\hat{\phi}(\tau, \vec{\sigma}), \Gamma\left(\tau, \vec{\sigma}^{\prime}\right)\right\}=\left\{\hat{\pi}_{\phi}(\tau, \vec{\sigma}), \Gamma\left(\tau, \vec{\sigma}^{\prime}\right)\right\}=0, \\
\left\{\hat{\phi}^{*}(\tau, \vec{\sigma}), \Gamma\left(\tau, \vec{\sigma}^{\prime}\right)\right\}=\left\{\hat{\pi}_{\phi^{*}}(\tau, \vec{\sigma}), \Gamma\left(\tau, \vec{\sigma}^{\prime}\right)\right\}=0, \\
\left\{\hat{\phi}(\tau, \vec{\sigma}), \hat{\pi}_{\phi}\left(\tau, \vec{\sigma}^{\prime}\right)\right\}=\left\{\hat{\phi}^{*}(\tau, \vec{\sigma}), \hat{\pi}_{\phi^{*}}\left(\tau, \vec{\sigma}^{\prime}\right)\right\}=\delta^{3}\left(\vec{\sigma}, \vec{\sigma}^{\prime}\right) .
\end{array}
$$

Therefore, in the generalized Wigner-covariant Coulomb gauge $A_{\tau}(\tau, \vec{\sigma})=\eta_{e m}(\tau, \vec{\sigma})=0$, we have $\hat{\phi}^{*}(\tau, \vec{\sigma})=[\hat{\phi}(\tau, \vec{\sigma})]^{*}, \hat{\pi}_{\phi^{*}}(\tau, \vec{\sigma})=\left[\hat{\pi}_{\phi}(\tau, \vec{\sigma})\right]^{*}$ like in the free case.

The constraints take the following form

$$
\begin{aligned}
\mathcal{H}(\tau) & =\epsilon_{s}-\left\{\frac{1}{2} \int d^{3} \sigma\left(\vec{\pi}_{\perp}^{2}+\vec{B}^{2}\right)(\tau, \vec{\sigma})+\right. \\
& +\int d^{3} \sigma\left[\hat{\pi}_{\phi^{*}} \hat{\pi}_{\phi}+\left(\vec{\partial}+i e \vec{A}_{\perp}\right) \hat{\phi}^{*} \cdot\left(\vec{\partial}-i e \vec{A}_{\perp}\right) \hat{\phi}+m^{2} \hat{\phi}^{*} \hat{\phi}\right](\tau, \vec{\sigma})- \\
& \left.-\frac{e^{2}}{8 \pi} \int d^{3} \sigma_{1} d^{3} \sigma_{2} \frac{i\left(\hat{\pi}_{\phi^{*}} \hat{\phi}^{*}-\hat{\pi}_{\phi} \hat{\phi}\right)\left(\tau, \vec{\sigma}_{1}\right) i\left(\hat{\pi}_{\phi^{*}} \hat{\phi}^{*}-\hat{\pi}_{\phi} \hat{\phi}\right)\left(\tau, \vec{\sigma}_{2}\right)}{\left|\vec{\sigma}_{1}-\vec{\sigma}_{2}\right|}\right\} \\
\overrightarrow{\mathcal{H}}_{p}(\tau) & =\int d^{3} \sigma\left(\vec{\pi}_{\perp} \times \vec{B}\right)(\tau, \vec{\sigma})+\int d^{3} \sigma\left(\hat{\pi}_{\phi^{*}} \vec{\partial} \hat{\phi}^{*}+\hat{\pi}_{\phi} \vec{\partial} \hat{\phi}\right)(\tau, \vec{\sigma}) \approx 0
\end{aligned}
$$

where the Coulomb self-interaction appears in the invariant mass and where the 3 constraints defining the rest frame do not depend on the interaction since we are in an instant form of the dynamics. The final form of the rest-frame spin tensor is

$$
\begin{aligned}
\bar{S}_{s}^{r s} & =\int d^{3} \sigma\left\{\sigma^{r}\left[\left(\vec{\pi}_{\perp} \times \vec{B}\right)^{s}+\hat{\pi}_{\phi^{*}} \partial^{s} \hat{\phi}^{*}+\hat{\pi}_{\phi} \partial^{s} \hat{\phi}\right]-(r \leftrightarrow s)\right\}(\tau, \vec{\sigma})= \\
& =S_{\phi}^{r s}(\tau)+\int d^{3} \sigma\left\{\sigma^{r}\left[\left(\vec{\pi}_{\perp} \times \vec{B}\right)^{s}-(r \leftrightarrow s)\right\}(\tau, \vec{\sigma}) .\right.
\end{aligned}
$$

If we go to the gauge $\chi=T_{s}-\tau \approx 0$, we can eliminate the variables $\epsilon_{s}, T_{s}$, and the $\tau$-evolution (in the Lorentz scalar rest-frame time) is governed by the Hamiltonian

$$
\begin{aligned}
H_{R} & =M-\vec{\lambda}(\tau) \cdot \overrightarrow{\mathcal{H}}_{p}(\tau) \\
M & =\frac{1}{2} \int d^{3} \sigma\left(\vec{\pi}_{\perp}^{2}+\vec{B}^{2}\right)(\tau, \vec{\sigma})+ \\
& +\int d^{3} \sigma\left[\hat{\pi}_{\phi^{*}} \hat{\pi}_{\phi}+\left(\vec{\partial}+i e \vec{A}_{\perp}\right) \hat{\phi}^{*} \cdot\left(\vec{\partial}-i e \vec{A}_{\perp}\right) \hat{\phi}+m^{2} \hat{\phi}^{*} \hat{\phi}\right](\tau, \vec{\sigma})- \\
& -\frac{e^{2}}{8 \pi} \int d^{3} \sigma_{1} d^{3} \sigma_{2} \frac{\left.i\left(\hat{\pi}_{\phi^{*}} \hat{\phi}^{*}-\hat{\pi}_{\phi} \hat{\phi}\right)\left(\tau, \vec{\sigma}_{1}\right) i\left(\hat{\pi}_{\phi^{*}} \hat{\phi}^{*}-\hat{\pi}_{\phi} \hat{\phi}\right)(\tau, \vec{\sigma})_{2}\right)}{\left|\vec{\sigma}_{1}-\vec{\sigma}_{2}\right|}
\end{aligned}
$$

In the gauge $\vec{\lambda}(\tau)=0$, the Hamilton equations are

$$
\begin{aligned}
& \partial_{\tau} \hat{\phi}(\tau, \vec{\sigma}) \stackrel{\circ}{=} \hat{\pi}_{\phi^{*}}(\tau, \vec{\sigma})+ \\
&+\frac{i e^{2}}{4 \pi} \hat{\phi}(\tau, \vec{\sigma}) \int d^{3} \bar{\sigma} \frac{i\left(\hat{\pi}_{\phi^{*}} \hat{\phi}^{*}-\hat{\pi}_{\phi} \hat{\phi}\right)(\tau, \vec{\sigma})}{|\vec{\sigma}-\overrightarrow{\vec{\sigma}}|}, \\
& \partial_{\tau} \hat{\pi}_{\phi^{*}}(\tau, \vec{\sigma}) \stackrel{\circ}{=}\left[\left(\vec{\partial}-i e \vec{A}_{\perp}(\tau, \vec{\sigma})\right)^{2}-m^{2}\right] \hat{\phi}(\tau, \vec{\sigma})+ \\
&+\frac{i e^{2}}{4 \pi} \hat{\pi}_{\phi^{*}}(\tau, \vec{\sigma}) \int d^{3} \overline{\bar{\sigma}} \frac{i\left(\hat{\pi}_{\phi^{*}} \hat{\phi}^{*}-\hat{\pi}_{\phi} \hat{\phi}\right)(\tau, \vec{\sigma})}{|\vec{\sigma}-\overrightarrow{\vec{\sigma}}|}
\end{aligned}
$$




$$
\begin{aligned}
\partial_{\tau} A_{\perp}^{r}(\tau, \vec{\sigma}) & \stackrel{\circ}{=}-\pi_{\perp}^{r}(\tau, \vec{\sigma}), \\
\partial_{\tau} \pi_{\perp}^{r}(\tau, \vec{\sigma}) & \stackrel{\circ}{=} \triangle A_{\perp}^{r}(\tau, \vec{\sigma})+ \\
& +i e P_{\perp}^{r s}(\vec{\sigma})\left[\hat{\phi}^{*}\left(\partial^{s}-i e A_{\perp}^{s}\right) \hat{\phi}-\hat{\phi}\left(\partial^{s}+i e A_{\perp}^{s}\right) \hat{\phi}^{*}\right](\tau, \vec{\sigma}) .
\end{aligned}
$$

The equations for $\hat{\phi}^{*}$ and $\pi_{\phi}$ are the complex conjugate of those for $\hat{\phi}$ and for $\hat{\pi}_{\phi^{*}}$.

By using the results of Ref. [21, we have the following inversion formula

$$
\begin{aligned}
& \hat{\pi}_{\phi^{*}} \stackrel{\circ}{=} \partial_{\tau} \hat{\phi}+i e^{2} \hat{\phi} \frac{1}{\triangle} i\left(\hat{\pi}_{\phi^{*}} \hat{\phi}^{*}-\hat{\pi}_{\phi} \hat{\phi}\right)= \\
& =\partial_{\tau} \hat{\phi}+i e^{2} \hat{\phi} \frac{1}{\triangle+2 e^{2} \hat{\phi}^{*} \hat{\phi}} i\left(\hat{\phi}^{*} \partial_{\tau} \hat{\phi}-\hat{\phi} \partial_{\tau} \hat{\phi}^{*}\right) \text {, }
\end{aligned}
$$

since we have $i\left(\hat{\phi}^{*} \partial_{\tau} \hat{\phi}-\hat{\phi} \partial_{\tau} \hat{\phi}^{*}\right)=\left(1+2 e^{2} \hat{\phi}^{*} \hat{\phi} \frac{1}{\triangle}\right) i\left(\hat{\pi}_{\phi^{*}} \hat{\phi}^{*}-\hat{\pi}_{\phi} \hat{\phi}\right)$ and where use has been done of the operator identity $\frac{1}{A} \frac{1}{1+B \frac{1}{A}}=\frac{1}{A}\left[1-B \frac{1}{A}+B \frac{1}{A} B \frac{1}{A}-\ldots\right]=\frac{1}{A+B}$ (valid for B a small perturbation of A) for $A=\triangle$ and $B=2 e^{2} \hat{\phi}^{*} \hat{\phi}$.

Using this formula, we get the following second order equations of motion

$$
\begin{aligned}
& \left\{\left[\partial_{\tau}+i e^{2} \frac{1}{\triangle+2 e^{2} \hat{\phi}^{*} \hat{\phi}} i\left(\hat{\phi}^{*} \partial_{\tau} \hat{\phi}-\hat{\phi} \partial_{\tau} \hat{\phi}^{*}\right)\right]^{2}-\left(\vec{\partial}-i e \vec{A}_{\perp}\right)^{2}+m^{2}\right\} \hat{\phi}^{\circ}=0, \\
& {\left[\partial_{\tau}^{2}+\triangle\right] A_{\perp}^{r} \stackrel{\circ}{=} i e P_{\perp}^{r s}(\vec{\sigma})\left[\hat{\phi}^{*}\left(\partial^{s}-i e A_{\perp}^{s}\right) \hat{\phi}-\hat{\phi}\left(\partial^{s}+i e A_{\perp}^{s}\right) \hat{\phi}^{*}\right] .}
\end{aligned}
$$

We see that the non-local velocity-dependent self-energy is formally playing the role of a scalar potential.

Due to Eqs. 8.31), in the gauge $A_{\tau}(\tau, \vec{\sigma})=\eta_{e m}(\tau, \vec{\sigma})=0$ the Dirac observables $\hat{\phi}, \hat{\pi}_{\phi}$, $\hat{\phi}^{*}, \hat{\pi}_{\phi^{*}}$, admit a Fourier decomposition like in the free case, see Eqs.(8.10) and (D5), with Fourier coefficients $\hat{a}, \hat{b}$ [the fields $\hat{\phi}_{a}, \hat{\phi}_{b}$ and the Fourier coefficients $\hat{a}, \hat{b}$, tend to the free ones $\phi_{a}, \phi_{b}, a, b$, when we turn off the electromagnetic interaction, switching off the electric charge]

$$
\begin{aligned}
\hat{a}(\tau, \vec{q}) & =\sqrt{2 m \omega(q)} \int d^{3} \sigma \hat{\phi}_{a}(\tau, \vec{\sigma}) e^{i(\omega(q) \tau-\vec{q} \cdot \vec{\sigma})}= \\
& =\int d^{3} \sigma\left[\omega(q) \hat{\phi}(\tau, \vec{\sigma})+i \hat{\pi}_{\phi^{*}}(\tau, \vec{\sigma})\right] e^{i(\omega(q) \tau-\vec{q} \cdot \vec{\sigma})}= \\
& =\sqrt{\hat{I}_{a}(\tau, \vec{q})} e^{i \hat{\varphi}_{a}(\tau, \vec{q})}, \\
\hat{a}^{*}(\tau, \vec{q}) & =\sqrt{2 m \omega(q)} \int d^{3} \sigma \hat{\phi}_{a}^{*}(\tau, \vec{\sigma}) e^{-i(\omega(q) \tau-\vec{q} \cdot \vec{\sigma})}= \\
& =\int d^{3} \sigma\left[\omega(q) \hat{\phi}^{*}(\tau, \vec{\sigma})+i \hat{\pi}_{\phi}(\tau, \vec{\sigma})\right] e^{-i(\omega(q) \tau-\vec{q} \cdot \vec{\sigma})}= \\
& =\sqrt{\hat{I}_{a}(\tau, \vec{q})} e^{-i \hat{\varphi}_{a}(\tau, \vec{q})}, \\
\hat{b}(\tau, \vec{q}) & =\sqrt{2 m \omega(q)} \int d^{3} \sigma \hat{\phi}_{b}(\tau, \vec{\sigma}) e^{i(\omega(q) \tau-\vec{q} \cdot \vec{\sigma})}= \\
& =\int d^{3} \sigma\left[\omega(q) \hat{\phi}(\tau, \vec{\sigma})-i \hat{\pi}_{\phi^{*}}(\tau, \vec{\sigma})\right] e^{i(\omega(q) \tau-\vec{q} \cdot \vec{\sigma})}= \\
& =\sqrt{\hat{I}_{b}(\tau, \vec{q})} e^{i \hat{\varphi}_{b}(\tau, \vec{q})},
\end{aligned}
$$




$$
\begin{aligned}
& \hat{b}^{*}(\tau, \vec{q})=\sqrt{2 m \omega(q)} \int d^{3} \sigma \hat{\phi}_{b}^{*}(\tau, \vec{\sigma}) e^{-i(\omega(q) \tau-\vec{q} \cdot \vec{\sigma})}= \\
& =\int d^{3} \sigma\left[\omega(q) \hat{\phi}^{*}(\tau, \vec{\sigma})+i \hat{\pi}_{\phi}(\tau, \vec{\sigma})\right] e^{-i(\omega(q) \tau-\vec{q} \cdot \vec{\sigma})}= \\
& =\sqrt{\hat{I}_{b}(\tau, \vec{q})} e^{-i \hat{\varphi}_{b}(\tau, \vec{q})}, \\
& \hat{\phi}_{a}(\tau, \vec{\sigma})=\frac{1}{\sqrt{2 m \sqrt{m^{2}+\triangle}}}\left[\sqrt{m^{2}+\triangle} \hat{\phi}(\tau, \vec{\sigma})+i \hat{\pi}_{\phi^{*}}(\tau, \vec{\sigma})\right]= \\
& =\sqrt{\frac{2}{m}} \int d \tilde{q} \sqrt{\omega(q)} \hat{a}(\tau, \vec{q}) e^{-i(\omega(q) \tau-\vec{q} \cdot \vec{\sigma})}, \\
& \hat{\phi}_{b}(\tau, \vec{\sigma})=\frac{1}{\sqrt{2 m \sqrt{m^{2}+\triangle}}}\left[\sqrt{m^{2}+\triangle} \hat{\phi}(\tau, \vec{\sigma})+i \hat{\pi}_{\phi^{*}}(\tau, \vec{\sigma})\right]= \\
& =\sqrt{\frac{2}{m}} \int d \tilde{q} \sqrt{\omega(q)} \hat{b}(\tau, \vec{q}) e^{-i(\omega(q) \tau-\vec{q} \cdot \vec{\sigma})}, \\
& \hat{\phi}(\tau, \vec{\sigma})=\left[\hat{\phi}^{*}(\tau, \vec{\sigma})\right]^{*}=\sqrt{\frac{m}{2}} \frac{1}{\left(m^{2}+\triangle\right)^{1 / 4}}\left[\hat{\phi}_{a}+\hat{\phi}_{b}^{*}\right](\tau, \vec{\sigma}), \\
& \hat{\pi}_{\phi^{*}}(\tau, \vec{\sigma})=\left[\hat{\pi}_{\phi}(\tau, \vec{\sigma})\right]^{*}=-i \sqrt{\frac{m}{2}}\left(m^{2}+\triangle\right)^{1 / 4}\left[\hat{\phi}_{a}-\hat{\phi}_{b}^{*}\right](\tau, \vec{\sigma}) .
\end{aligned}
$$

Let us now assume that the fields satisfy Eq.(4.29). Then, we can define the canonical transformations $\hat{a}, \hat{a}^{*} \mapsto \hat{P}_{a \phi}^{A}, \hat{X}_{a \phi}^{A}\left(\hat{X}_{a \phi}^{\tau}=\tau+\hat{\tilde{X}}_{a \phi}\right), \hat{\mathbf{H}}_{a}, \hat{\mathbf{K}}_{a}$ and $\hat{b}, \hat{b}^{*} \mapsto \hat{P}_{b \phi}^{A}, \hat{X}_{b \phi}^{A}\left(\hat{X}_{b \phi}^{\tau}=\right.$ $\left.\tau+\hat{\tilde{X}}_{b \phi}^{\tau}\right), \hat{\mathbf{H}}_{b}, \hat{\mathbf{K}}_{b}$, and then $\hat{X}_{a \phi}^{A}, \hat{P}_{a \phi}^{A}, \hat{X}_{b \phi}^{A}, \hat{P}_{b \phi}^{A} \mapsto \hat{X}_{\phi}^{A}, \hat{P}_{\phi}^{A}, \hat{R}_{\phi}^{A}, \hat{Q}_{\phi}^{A}$. We get

$$
\begin{aligned}
& \hat{P}_{\phi}^{\tau}=m \int d^{3} \sigma\left[\hat{\phi}_{a}^{*} \sqrt{m^{2}+\triangle} \hat{\phi}_{a}+\hat{\phi}_{b}^{*} \sqrt{m^{2}+\triangle} \hat{\phi}_{b}\right](\tau, \vec{\sigma}), \\
& \hat{\vec{P}}_{\phi}=i m \int d^{3} \sigma\left[\hat{\phi}_{a}^{*} \vec{\partial} \hat{\phi}_{a}+\hat{\phi}_{b}^{*} \vec{\partial} \hat{\phi}_{b}\right](\tau, \vec{\sigma}), \\
& \hat{Q}_{\phi}^{\tau}=\frac{m}{2} \int d^{3} \sigma\left[\hat{\phi}_{a}^{*} \sqrt{m^{2}+\triangle} \hat{\phi}_{a}-\hat{\phi}_{b}^{*} \sqrt{m^{2}+\triangle} \hat{\phi}_{b}\right](\tau, \vec{\sigma}), \\
& \hat{\vec{Q}}_{\phi}=i \frac{m}{2} \int d^{3} \sigma\left[\hat{\phi}_{a}^{*} \vec{\partial} \hat{\phi}_{a}-\hat{\phi}_{b}^{*} \vec{\partial} \hat{\phi}_{b}\right](\tau, \vec{\sigma}), \\
& \hat{X}_{\phi}^{\tau}=\tau+\hat{\tilde{X}}_{\phi}^{\tau}=\tau+ \\
& +\frac{1}{4 \pi i m} \int d^{3} q \frac{e^{-\frac{4 \pi}{m^{2}} q^{2}}}{q^{2} \omega(q)} \\
& \left(\ln \frac{\int d^{3} \sigma e^{i \vec{q} \cdot \vec{\sigma}}\left(m^{2}+\triangle\right)^{-1 / 4}\left[\left(\omega(q)+\sqrt{m^{2}+\triangle}\right) \hat{\phi}_{a}+\left(\omega(q)-\sqrt{m^{2}+\triangle}\right) \hat{\phi}_{b}^{*}\right](\tau, \vec{\sigma})}{\int d^{3} \sigma e^{-i \vec{q} \cdot \vec{\sigma}}\left(m^{2}+\triangle\right)^{-1 / 4}\left[\left(\omega(q)+\sqrt{m^{2}+\triangle}\right) \hat{\phi}_{a}^{*}+\left(\omega(q)-\sqrt{m^{2}+\triangle}\right) \hat{\phi}_{b}\right](\tau, \vec{\sigma})}+\right. \\
& +\ln \frac{\int d^{3} \sigma e^{i \vec{q} \cdot \vec{\sigma}}\left(m^{2}+\triangle\right)^{-1 / 4}\left[\left(\omega(q)+\sqrt{m^{2}-\triangle}\right) \hat{\phi}_{a}^{*}+\left(\omega(q)+\sqrt{m^{2}+\triangle}\right) \hat{\phi}_{b}\right](\tau, \vec{\sigma})}{\int d^{3} \sigma e^{-i \vec{q} \cdot \vec{\sigma}}\left(m^{2}+\triangle\right)^{-1 / 4}\left[\left(\omega(q)-\sqrt{m^{2}+\triangle}\right) \hat{\phi}_{a}+\left(\omega(q)+\sqrt{m^{2}+\triangle}\right) \hat{\phi}_{b}^{*}\right](\tau, \vec{\sigma})}, \\
& \hat{\vec{X}}_{\phi}=\frac{2 i}{\pi m} \int d^{3} q \frac{\vec{q}}{q^{4}} e^{-\frac{4 \pi}{m^{2}} q^{2}} \\
& \left(\ln \frac{\int d^{3} \sigma e^{i \vec{q} \cdot \vec{\sigma}}\left(m^{2}+\triangle\right)^{-1 / 4}\left[\left(\omega(q)+\sqrt{m^{2}+\triangle}\right) \hat{\phi}_{a}+\left(\omega(q)-\sqrt{m^{2}+\triangle}\right) \hat{\phi}_{b}^{*}\right](\tau, \vec{\sigma})}{\int d^{3} \sigma e^{-i \vec{q} \cdot \vec{\sigma}}\left(m^{2}+\triangle\right)^{-1 / 4}\left[\left(\omega(q)+\sqrt{m^{2}+\triangle}\right) \hat{\phi}_{a}^{*}+\left(\omega(q)-\sqrt{m^{2}+\triangle}\right) \hat{\phi}_{b}\right](\tau, \vec{\sigma})}-\right.
\end{aligned}
$$




$$
\begin{aligned}
& -\ln \frac{\int d^{3} \sigma e^{i \vec{q} \cdot \vec{\sigma}}\left(m^{2}+\triangle\right)^{-1 / 4}\left[\left(\omega(q)+\sqrt{m^{2}-\triangle}\right) \hat{\phi}_{a}^{*}+\left(\omega(q)+\sqrt{m^{2}+\triangle}\right) \hat{\phi}_{b}\right](\tau, \vec{\sigma})}{\int d^{3} \sigma e^{-i \vec{q} \cdot \vec{\sigma}}\left(m^{2}+\triangle\right)^{-1 / 4}\left[\left(\omega(q)-\sqrt{m^{2}+\triangle}\right) \hat{\phi}_{a}+\left(\omega(q)+\sqrt{m^{2}+\triangle}\right) \hat{\phi}_{b}^{*}\right](\tau, \vec{\sigma})}, \\
& \hat{R}_{\phi}^{\tau}=\frac{1}{2 \pi i m} \int d^{3} q \frac{e^{-\frac{4 \pi}{m^{2}} q^{2}}}{q^{2} \omega(q)} \\
& \left(\ln \frac{\int d^{3} \sigma e^{i \vec{q} \cdot \vec{\sigma}}\left(m^{2}+\triangle\right)^{-1 / 4}\left[\left(\omega(q)+\sqrt{m^{2}+\triangle}\right) \hat{\phi}_{a}+\left(\omega(q)-\sqrt{m^{2}+\triangle}\right) \hat{\phi}_{b}^{*}\right](\tau, \vec{\sigma})}{\int d^{3} \sigma e^{-i \vec{q} \cdot \vec{\sigma}}\left(m^{2}+\triangle\right)^{-1 / 4}\left[\left(\omega(q)+\sqrt{m^{2}+\triangle}\right) \hat{\phi}_{a}^{*}+\left(\omega(q)-\sqrt{m^{2}+\triangle}\right) \hat{\phi}_{b}\right](\tau, \vec{\sigma})}-\right. \\
& -\ln \frac{\int d^{3} \sigma e^{i \vec{q} \cdot \vec{\sigma}}\left(m^{2}+\triangle\right)^{-1 / 4}\left[\left(\omega(q)+\sqrt{m^{2}-\triangle}\right) \hat{\phi}_{a}^{*}+\left(\omega(q)+\sqrt{m^{2}+\triangle}\right) \hat{\phi}_{b}\right](\tau, \vec{\sigma})}{\int d^{3} \sigma e^{-i \vec{q} \cdot \vec{\sigma}}\left(m^{2}+\triangle\right)^{-1 / 4}\left[\left(\omega(q)-\sqrt{m^{2}+\triangle}\right) \hat{\phi}_{a}+\left(\omega(q)+\sqrt{m^{2}+\triangle}\right) \hat{\phi}_{b}^{*}\right](\tau, \vec{\sigma})}, \\
& \hat{\vec{R}}_{\phi}=\frac{i}{\pi m} \int d^{3} q \frac{\vec{q}}{q^{4}} e^{-\frac{4 \pi}{m^{2}} q^{2}} \\
& \left(\ln \frac{\int d^{3} \sigma e^{i \vec{q} \cdot \vec{\sigma}}\left(m^{2}+\triangle\right)^{-1 / 4}\left[\left(\omega(q)+\sqrt{m^{2}+\triangle}\right) \hat{\phi}_{a}+\left(\omega(q)-\sqrt{m^{2}+\triangle}\right) \hat{\phi}_{b}^{*}\right](\tau, \vec{\sigma})}{\int d^{3} \sigma e^{-i \vec{q} \cdot \vec{\sigma}}\left(m^{2}+\triangle\right)^{-1 / 4}\left[\left(\omega(q)+\sqrt{m^{2}+\triangle}\right) \hat{\phi}_{a}^{*}+\left(\omega(q)-\sqrt{m^{2}+\triangle}\right) \hat{\phi}_{b}\right](\tau, \vec{\sigma})}+\right. \\
& +\ln \frac{\int d^{3} \sigma e^{i \vec{q} \cdot \vec{\sigma}}\left(m^{2}+\triangle\right)^{-1 / 4}\left[\left(\omega(q)+\sqrt{m^{2}-\triangle}\right) \hat{\phi}_{a}^{*}+\left(\omega(q)+\sqrt{m^{2}+\triangle}\right) \hat{\phi}_{b}\right](\tau, \vec{\sigma})}{\int d^{3} \sigma e^{-i \vec{q} \cdot \vec{\sigma}}\left(m^{2}+\triangle\right)^{-1 / 4}\left[\left(\omega(q)-\sqrt{m^{2}+\triangle}\right) \hat{\phi}_{a}+\left(\omega(q)+\sqrt{m^{2}+\triangle}\right) \hat{\phi}_{b}^{*}\right](\tau, \vec{\sigma})}, \\
& \hat{\phi}_{a}(\tau, \vec{\sigma})=\int d \tilde{k} \sqrt{\frac{2 \omega(k)}{m}} \mathbf{A}_{a \vec{k}}\left(\tau ; \frac{1}{2} \hat{P}_{\phi}^{A}+\hat{Q}_{\phi}^{A}, \hat{\mathbf{B}}_{a}\right] e^{i\left(\vec{k} \cdot \vec{\sigma}+\mathbf{B}_{a \vec{k}}\left(\tau ; \hat{X}_{\phi}^{A}+\frac{1}{2} \hat{R}_{\phi}^{A}, \hat{\mathbf{K}}_{a}\right]\right)}, \\
& \hat{\phi}_{b}(\tau, \vec{\sigma})=\int d \tilde{k} \sqrt{\frac{2 \omega(k)}{m}} \mathbf{A}_{b \vec{k}}\left(\tau ; \frac{1}{2} \hat{P}_{\phi}^{A}+\hat{Q}_{\phi}^{A}, \hat{\mathbf{B}}_{b}\right] e^{i\left(\vec{k} \cdot \vec{\sigma}+\mathbf{B}_{b \vec{k}}\left(\tau ; \hat{X}_{\phi}^{A}+\frac{1}{2} \hat{R}_{\phi}^{A}, \hat{\mathbf{K}}_{b}\right]\right)} .
\end{aligned}
$$

In terms of these variables Eqs. (8.34) and (8.33) become

$$
\begin{aligned}
H_{R}= & M-\vec{\lambda}(\tau) \cdot \overrightarrow{\mathcal{H}}_{p}(\tau) \\
M= & \frac{1}{2} \int d^{3} \sigma\left(\vec{\pi}_{\perp}^{2}+\vec{B}^{2}\right)(\tau, \vec{\sigma})+\hat{P}_{\phi}^{\tau}(\tau)+ \\
+ & m \int d^{3} \sigma\left[i e \vec{A}_{\perp}(\tau, \vec{\sigma}) \cdot \frac{1}{\sqrt{m^{2}+\triangle^{2}}}\left[\hat{\phi}_{a}^{*}+\hat{\phi}_{b}\right](\tau, \vec{\sigma}) \frac{\vec{\partial}}{\sqrt{m^{2}+\triangle^{2}}}\left[\hat{\phi}_{a}+\hat{\phi}_{b}^{*}\right](\tau, \vec{\sigma})+\right. \\
+ & \left.\frac{e^{2}}{2} \vec{A}_{\perp}^{2}(\tau, \vec{\sigma}) \frac{1}{\sqrt{m^{2}+\triangle}}\left[\hat{\phi}_{a}^{*}+\hat{\phi}_{b}\right](\tau, \vec{\sigma}) \frac{1}{\sqrt{m^{2}+\triangle}}\left[\hat{\phi}_{a}+\hat{\phi}_{b}^{*}\right](\tau, \vec{\sigma})\right]- \\
- & \frac{e^{2} m^{2}}{32 \pi} \int \frac{d^{3} \sigma_{1} d^{3} \sigma_{2}}{\left|\vec{\sigma}_{1}-\vec{\sigma}_{2}\right|}\left[\sqrt{m^{2}+\triangle_{1}}\left[\hat{\phi}_{a}-\hat{\phi}_{b}^{*}\right] \frac{1}{\sqrt{m^{2}+\triangle_{1}}}\left[\hat{\phi}_{a}^{*}+\hat{\phi}_{b}\right]-\right. \\
- & \left.\sqrt{m^{2}+\triangle_{1}}\left[\hat{\phi}_{a}^{*}-\hat{\phi}_{b}\right] \frac{1}{\sqrt{m^{2}+\triangle_{1}}}\left[\hat{\phi}_{a}+\hat{\phi}_{b}^{*}\right]\right]\left(\tau, \vec{\sigma}_{1}\right) \\
& {\left[\sqrt{m^{2}+\triangle_{2}}\left[\hat{\phi}_{a}-\hat{\phi}_{b}^{*}\right] \frac{1}{\sqrt{m^{2}+\triangle_{2}}}\left[\hat{\phi}_{a}^{*}+\hat{\phi}_{b}\right]-\right.} \\
- & \left.\sqrt{m^{2}+\triangle_{2}}\left[\hat{\phi}_{a}^{*}-\hat{\phi}_{b}\right] \frac{1}{\sqrt{m^{2}+\triangle_{2}}}\left[\hat{\phi}_{a}+\hat{\phi}_{b}^{*}\right]\right]\left(\tau, \vec{\sigma}_{2}\right), \\
\overrightarrow{\mathcal{H}}_{p}(\tau)= & \hat{\vec{P}}_{\phi}(\tau)+\int d^{3} \sigma\left(\vec{\pi}_{\perp} \times \vec{B}\right)(\tau, \vec{\sigma}) \approx 0, \\
\bar{S}_{s}^{r s} \equiv & \hat{S}_{\phi}^{r s}(\tau)+\int d^{3} \sigma\left[\sigma^{r}\left(\vec{\pi}_{\perp} \times \vec{B}\right)^{s}-\sigma^{s}\left(\vec{\pi}_{\perp} \times \vec{B}\right)^{r}\right] .
\end{aligned}
$$


In Appendix Ethere is the expression of the interaction terms in $\mathrm{M}$ in terms of $\hat{a}, \hat{b}$.

The fields $\hat{\phi}_{a}, \hat{\phi}_{b}$, are solutions of the coupled Hamilton equations generated by $H_{R}$ and these equations cannot be decoupled when the associated Feshbach-Villars Hamiltonian cannot be diagonalized: in these cases $\hat{\phi}_{a}\left[\hat{\phi}_{b}\right]$ does not correspond to a positive [negative] energy solution.

Only when we have the collective and relative canonical variables of the transverse electromagnetic fields $\vec{A}_{\perp}, \vec{\pi}_{\perp}$, we will be able to add the gauge fixings for the constraints $\overrightarrow{\mathcal{H}}_{p} \approx 0$, defining the rest frame, and to get the final form of the physical Hamiltonian for classical scalar electrodynamics in the rest-frame Wigner-covariant instant form of the dynamics.

\section{The energy-momentum tensor.}

The conserved energy-momentum tensor of the isolated system formed by the KleinGordon field plus the electromagnetic field is $\left[D_{\mu}^{(A)} \tilde{\phi}(x)=\left[\partial_{\mu}-i e A_{\mu}(x)\right] \tilde{\phi}(x) ; \tilde{\phi}(z(\tau, \vec{\sigma}))=\right.$ $\left.\phi(\tau, \vec{\sigma}) ; \tilde{A}_{\mu}(z(\tau, \vec{\sigma}))=z_{\mu}^{A}(\tau, \vec{\sigma}) A_{A}(\tau, \vec{\sigma}) ; T_{e m}^{\mu \nu}(x)=\tilde{F}^{\mu \alpha}(x) \tilde{F}_{\alpha}^{\nu}(x)+\frac{1}{4} \eta^{\mu \nu} \tilde{F}^{\alpha \beta}(x) \tilde{F}_{\alpha \beta}(x)\right]$

$$
\begin{aligned}
\Theta^{\mu \nu}(x) & =\tilde{F}^{\mu \alpha}(x) \tilde{F}_{\alpha}{ }^{\nu}(x)+\frac{1}{4} \eta^{\mu \nu} \tilde{F}^{\alpha \beta}(x) \tilde{F}_{\alpha \beta}(x)+ \\
& +\left(D^{(A) \mu} \tilde{\phi}(x)\right)^{*} D^{(A) \nu} \tilde{\phi}(x)+\left(D^{(A) \nu} \tilde{\phi}(x)\right)^{*} D^{(A) \mu} \tilde{\phi}(x)- \\
& -\eta^{\mu \nu}\left[\left(D^{(A) \alpha} \tilde{\phi}(x)\right)^{*} D^{(A)} \tilde{\phi}(x)\right]= \\
& =\left[z _ { A } ^ { \mu } z _ { B } ^ { \nu } \left(\left[g^{A D} g^{C E} g^{B F}+\frac{1}{4} g^{A B} g^{C D} g^{E F}\right] F_{D E} F_{C F}+g^{A B} m^{2} \phi^{*} \phi+\right.\right. \\
& \left.\left.+\left[g^{A C} g^{B D}+g^{B C} g^{A D}-g^{A B} g^{C D}\right]\left(\partial_{C}+i e A_{C}\right) \phi^{*}\left(\partial_{D}-i e A_{D}\right) \phi\right)\right](\tau, \vec{\sigma})= \\
& =T_{e m}^{\mu \nu}(x)+T_{\phi, A}^{\mu \nu}(x), \\
\partial_{\nu} \Theta^{\nu \mu}(x) & \stackrel{\circ}{=} 0 \\
\Rightarrow & \partial_{\nu} T_{\phi, A}^{\mu \nu}(x) \stackrel{\circ}{=}-\tilde{F}^{\mu \nu}(x) J_{\phi \nu}(x),
\end{aligned}
$$

where the conserved electromagnetic current of the Klein-Gordon field $J_{\phi}^{\mu}$ is $\left[\partial_{\mu} J_{\phi}^{\mu} \stackrel{\circ}{=} 0\right.$ is replaced by $\frac{d q_{\phi}}{d T_{s}} \stackrel{\circ}{=} 0$ at the Hamiltonian level on the Wigner hyperplanes with $q_{\phi}=e$ given in Eq.(8.11)]

$$
\begin{aligned}
J_{\phi}^{\mu}(\tau, \vec{\sigma}) & =-i\left[\left(\partial^{\mu}+i e \tilde{A}^{\mu}\right) \tilde{\phi}^{*} \tilde{\phi}-\tilde{\phi}^{*}\left(\partial^{\mu}-i e \tilde{A}^{\mu}\right) \tilde{\phi}\right](z)= \\
& =-i z_{A}^{\mu}(\tau, \vec{\sigma})\left[\left(\partial^{A}+i e A^{A}\right) \phi^{*} \phi-\phi^{*}\left(\partial^{A}-i e A^{A}\right) \phi\right](\tau, \vec{\sigma})= \\
& =i z_{\tau}^{\mu}(\tau, \vec{\sigma})\left[\pi_{\phi^{*}} \phi^{*}-\pi_{\phi} \phi\right](\tau, \vec{\sigma})+ \\
& +i z_{r}^{\mu}(\tau, \vec{\sigma})\left[\phi^{*}\left(\partial^{r}-i e A^{r}\right) \phi-\left(\partial^{r}+i e A^{r}\right) \phi^{*} \phi\right](\tau, \vec{\sigma}) .
\end{aligned}
$$

On Wigner hyperplanes with $T_{s}=\tau$ in the Coulomb gauge we have

$$
\begin{aligned}
\hat{J}_{\phi}^{\mu}\left(T_{s}, \vec{\sigma}\right) & =u^{\mu}\left(p_{s}\right) i\left[\hat{\pi}_{\phi^{*}} \hat{\phi}^{*}-\hat{\pi}_{\phi} \hat{\phi}\right]\left(T_{s}, \vec{\sigma}\right)+ \\
& \left.+\epsilon_{r}^{\mu}\left(u\left(p_{s}\right)\right) i\left[\hat{\phi}^{*}\left(\partial^{r}-i e A_{\perp}^{r}\right) \hat{\phi}-\left(\partial^{r}+i e A_{\perp}^{r}\right) \hat{\phi}^{*} \hat{\phi}\right]\left(T_{s}, \vec{\sigma}\right)\right] .
\end{aligned}
$$

On the Wigner hyperplanes we get the following expression for the energy-momentum tensor 


$$
\begin{aligned}
\Theta^{\mu \nu} & {\left[x_{s}^{\beta}\left(T_{s}\right)+\epsilon_{u}^{\beta}\left(u\left(p_{s}\right)\right) \sigma^{u}\right]=} \\
= & u^{\mu}\left(p_{s}\right) u^{\nu}\left(p_{s}\right)\left[\frac{1}{2}\left(\vec{\pi}^{2}+\vec{B}^{2}\right)+\pi_{\phi^{*}} \pi_{\phi}+(\vec{\partial}+i e \vec{A}) \phi^{*} \cdot(\vec{\partial}-i e \vec{A}) \phi+m^{2} \phi^{*} \phi\right]\left(T_{s}, \vec{\sigma}\right)+ \\
+ & \epsilon_{r}^{\mu}\left(u\left(p_{s}\right)\right) \epsilon_{s}^{\nu}\left(u\left(p_{s}\right)\right)\left[\frac{1}{2} \delta^{r s}\left(\vec{\pi}^{2}+\vec{B}^{2}\right)-\left(\pi^{r} \pi^{s}+B^{r} B^{s}\right)+\delta^{r s}\left(\pi_{\phi^{*}} \pi_{\phi}-m^{2} \phi^{*} \phi\right)+\right. \\
+ & \left.\left(\delta^{r u} \delta^{s v}+\delta^{r v} \delta^{s u}-\delta^{r s} \delta^{u v}\right)\left(\partial^{u}+i e A^{u}\right) \phi^{*}\left(\partial^{v}-i e A^{v}\right) \phi\right]\left(T_{s}, \vec{\sigma}\right)+ \\
+ & {\left[u^{\mu}\left(p_{s}\right) \epsilon_{r}^{\nu}\left(u\left(p_{s}\right)\right)+u^{\nu}\left(p_{s}\right) \epsilon_{r}^{\mu}\left(u\left(p_{s}\right)\right)\right] } \\
& {\left[(\vec{\pi} \times \vec{B})^{r}+\pi_{\phi^{*}}\left(\partial^{r}+i e A^{r}\right) \phi^{*}+\pi_{\phi}\left(\partial^{r}-i e A^{r}\right) \phi\right]\left(T_{s}, \vec{\sigma}\right), }
\end{aligned}
$$

whose expression in the $A_{\tau}\left(T_{s}, \vec{\sigma}\right)=\eta_{e m}\left(T_{s}, \vec{\sigma}\right)=0$ Coulomb gauge, where, from Eq.(3.28), we have $\pi^{r}=\pi_{\perp}^{r}+\frac{e}{\triangle} \hat{\mathcal{Q}}$ with $\hat{\mathcal{Q}}=i\left[\hat{\pi}_{\phi^{*}} \hat{\phi}^{*}-\hat{\pi}_{\phi} \hat{\phi}\right]$ [so that Eq. (8.11]) becomes $q_{\phi}=$ $\left.\int d^{3} \sigma \hat{\mathcal{Q}}(\tau, \vec{\sigma})\right]$, is

$$
\begin{aligned}
& \hat{\Theta}^{\mu \nu} \quad\left[x_{s}^{\beta}\left(T_{s}\right)+\epsilon_{u}^{\beta}\left(u\left(p_{s}\right)\right) \sigma^{u}\right]= \\
& =u^{\mu}\left(p_{s}\right) u^{\nu}\left(p_{s}\right)\left[\frac{1}{2}\left(\vec{\pi}_{\perp}^{2}+\vec{B}^{2}\right)+e \vec{\pi}_{\perp} \cdot \frac{\vec{\partial}}{\triangle} \hat{\mathcal{Q}}+\frac{e^{2}}{2}\left(\frac{\vec{\partial}}{\triangle} \hat{\mathcal{Q}}\right)^{2}+\right. \\
& \left.+\hat{\pi}_{\phi^{*}} \hat{\pi}_{\phi}+\left(\vec{\partial}+i e \vec{A}_{\perp}\right) \hat{\phi}^{*} \cdot\left(\vec{\partial}-i e \vec{A}_{\perp}\right) \hat{\phi}+m^{2} \hat{\phi}^{*} \hat{\phi}\right]\left(T_{s}, \vec{\sigma}\right)+ \\
& +\epsilon_{r}^{\mu}\left(u\left(p_{s}\right)\right) \epsilon_{s}^{\nu}\left(u\left(p_{s}\right)\right)\left[\frac{1}{2} \delta^{r s}\left(\vec{\pi}_{\perp}^{2}+\vec{B}^{2}\right)-\left(\pi_{\perp}^{r} \pi_{\perp}^{s}+B^{r} B^{s}\right)+\right. \\
& +e\left(\delta^{r s} \vec{\pi}_{\perp} \cdot \frac{\vec{\partial}}{\triangle} \hat{\mathcal{Q}}-\left[\pi_{\perp}^{r} \frac{\partial^{s}}{\triangle}+\pi_{\perp}^{s} \frac{\partial^{r}}{\triangle} \hat{\mathcal{Q}}\right)+\right. \\
& +e^{2}\left(\frac{1}{2} \delta^{r s}\left(\frac{\vec{\partial}}{\triangle} \hat{\mathcal{Q}}\right)^{2}-\frac{\partial^{r}}{\triangle} \hat{\mathcal{Q}} \frac{\partial^{s}}{\triangle} \hat{\mathcal{Q}}\right)+ \\
& +\delta^{r s}\left(\hat{\pi}_{\phi^{*}} \hat{\pi}_{\phi}-m^{2} \hat{\phi}^{*} \hat{\phi}\right)+ \\
& \left.+\left(\delta^{r u} \delta^{s v}+\delta^{r v} \delta^{s u}-\delta^{r s} \delta^{u v}\right)\left(\partial^{u}+i e A_{\perp}^{u}\right) \hat{\phi}^{*}\left(\partial^{v}-i e A_{\perp}^{v}\right) \hat{\phi}\right]\left(T_{s}, \vec{\sigma}\right)+ \\
& +\left[u^{\mu}\left(p_{s}\right) \epsilon_{r}^{\nu}\left(u\left(p_{s}\right)\right)+u^{\nu}\left(p_{s}\right) \epsilon_{r}^{\mu}\left(u\left(p_{s}\right)\right)\right] \\
& {\left[\left(\vec{\pi}_{\perp} \times \vec{B}\right)^{r}+\hat{\pi}_{\phi^{*}}\left(\partial^{r}+i e A_{\perp}^{r}\right) \hat{\phi}^{*}+\hat{\pi}_{\phi}\left(\partial^{r}-i e A_{\perp}^{r}\right) \hat{\phi}\right]\left(T_{s}, \vec{\sigma}\right),} \\
& \hat{\Theta}_{\mu}^{\mu} \quad\left[x_{s}^{\beta}\left(T_{s}\right)+\epsilon_{u}^{\beta}\left(u\left(p_{s}\right)\right) \sigma^{u}\right]= \\
& =-2\left[\hat{\pi}_{\phi^{*}} \hat{\pi}_{\phi}-\left(\vec{\partial}+i e \vec{A}_{\perp}\right) \hat{\phi}^{*} \cdot\left(\vec{\partial}-i e \vec{A}_{\perp}\right) \hat{\phi}-2 m^{2} \hat{\phi}^{*} \hat{\phi}\right]\left(T_{s}, \vec{\sigma}\right), \\
& \hat{P}_{\Theta}^{\mu}=\int d^{3} \sigma \hat{\Theta}^{\mu \nu} u_{\nu}\left(p_{s}\right)= \\
& =M u^{\mu}\left(p_{s}\right)+\mathcal{H}_{p}^{r}\left(T_{s}\right) \epsilon_{r}^{\mu}\left(u\left(p_{s}\right)\right) \approx M u^{\mu}\left(p_{s}\right), \\
& \hat{\Theta}_{S}^{r s}=\epsilon_{\mu}^{r}\left(u\left(p_{s}\right)\right) \epsilon_{\nu}^{s}\left(u\left(p_{s}\right)\right) \hat{\Theta}^{\mu \nu}= \\
& =\left[\frac{1}{2} \delta^{r s}\left(\vec{\pi}_{\perp}^{2}+\vec{B}^{2}\right)-\left(\pi_{\perp}^{r} \pi_{\perp}^{s}+B^{r} B^{s}\right)+\right. \\
& +e\left(\delta^{r s} \vec{\pi}_{\perp} \cdot \frac{\vec{\partial}}{\triangle} \hat{\mathcal{Q}}-\left[\pi_{\perp}^{r} \frac{\partial^{s}}{\triangle}+\pi_{\perp}^{s} \frac{\partial^{r}}{\triangle} \hat{\mathcal{Q}}\right)+\right. \\
& +e^{2}\left(\frac{1}{2} \delta^{r s}\left(\frac{\vec{\partial}}{\triangle} \hat{\mathcal{Q}}\right)^{2}-\frac{\partial^{r}}{\triangle} \hat{\mathcal{Q}} \frac{\partial^{s}}{\triangle} \hat{\mathcal{Q}}\right)+
\end{aligned}
$$




$$
\begin{aligned}
& +\delta^{r s}\left(\hat{\pi}_{\phi^{*}} \hat{\pi}_{\phi}-m^{2} \hat{\phi}^{*} \hat{\phi}\right)+ \\
& \left.+\left(\delta^{r u} \delta^{s v}+\delta^{r v} \delta^{s u}-\delta^{r s} \delta^{u v}\right)\left(\partial^{u}+i e A_{\perp}^{u}\right) \hat{\phi}^{*}\left(\partial^{v}-i e A_{\perp}^{v}\right) \hat{\phi}\right]\left(T_{s}, \vec{\sigma}\right) .
\end{aligned}
$$

The Dixon multipoles with respect to the origin $x_{s}^{\mu}(\tau)$ are

$$
\begin{aligned}
t_{\Theta}^{\mu_{1} \ldots \mu_{n} \mu \nu}\left(T_{s}\right) & =\int d^{3} \sigma \delta x_{s}^{\mu_{1}}(\vec{\sigma}) \ldots \delta x_{s}^{\mu_{n}}(\vec{\sigma}) \hat{\Theta}^{\mu \nu}\left[x_{s}^{\beta}\left(T_{s}\right)+\epsilon_{u}^{\beta}\left(u\left(p_{s}\right)\right) \sigma^{u}\right]= \\
& =\epsilon_{r_{1}}^{\mu_{1}}\left(u\left(p_{s}\right)\right) \ldots \epsilon_{r_{n}}^{\mu_{n}}\left(u\left(p_{s}\right)\right) \epsilon_{A}^{\mu}\left(u\left(p_{s}\right)\right) \epsilon_{B}^{\nu}\left(u\left(p_{s}\right)\right) I_{\Theta}^{r_{1} . . r_{n} A B}\left(T_{s}\right)
\end{aligned}
$$

and all the derived multipoles for the interacting case can be obtained from these ones following the scheme of Section $\mathrm{V}$.

With the same techniques we can study the multipoles $t_{\phi, A}^{\mu_{1} \ldots \mu_{n} \mu \nu}\left(T_{s}\right)$ and all the related ones $\hat{P}_{\phi, A}^{\mu}, \hat{S}_{\phi, A}^{\mu \nu}, \hat{J}_{\phi, A}^{\mu_{1} \ldots \mu_{n} \mu \nu}$ of $\hat{T}_{\phi, A}^{\mu \nu}$. The Dixon multipoles of the electromagnetic field in the rest-frame instant form will be studied in a future paper.

Let us remark that in absence of the electromagnetic field, namely in the free theory of a complex Klein-Gordon field, we have

$$
\begin{aligned}
\Theta^{\mu \nu}\left[x_{s}^{\beta}\left(T_{s}\right)+\epsilon_{u}^{\beta}\left(u\left(p_{s}\right)\right) \sigma^{u}\right] & =T_{\phi}^{\mu \nu}\left[x_{s}^{\beta}\left(T_{s}\right)+\epsilon_{u}^{\beta}\left(u\left(p_{s}\right)\right) \sigma^{u}\right]= \\
& =u^{\mu}\left(p_{s}\right) u^{\nu}\left(p_{s}\right)\left[\pi_{\phi^{*}} \pi_{\phi}+\vec{\partial} \phi^{*} \cdot \vec{\partial} \phi+m^{2} \phi^{*} \phi\right]\left(T_{s}, \vec{\sigma}\right)+ \\
& +\left[u^{\mu}\left(p_{s}\right) \epsilon_{r}^{\nu}\left(u\left(p_{s}\right)\right)+u^{\nu}\left(p_{s}\right) \epsilon_{r}^{\mu}\left(u\left(p_{s}\right)\right)\right]\left[\pi_{\phi^{*}} \partial^{r} \phi^{*}+\pi_{\phi} \partial^{r} \phi\right]\left(T_{s}, \vec{\sigma}\right)+ \\
& +\epsilon^{\mu}\left(u\left(p_{s}\right)\right) \epsilon_{s}^{\nu}\left(u\left(p_{s}\right)\right)\left[\delta^{r s}\left(\pi_{\phi^{*}} \pi_{\phi}-m^{2} \phi^{*} \phi\right)+\right. \\
& \left.+\left(\delta^{r u} \delta^{s v}+\delta^{r v} \delta^{s u}-\delta^{r s} \delta^{u v}\right) \partial^{u} \phi^{*} \partial^{v} \phi\right]\left(T_{s}, \vec{\sigma}\right), \\
t_{T *}^{\mu_{1} \ldots \mu_{n} \mu \nu}\left(T_{s}\right) & =\int d^{3} \sigma \delta x_{s}^{\mu_{1}}(\vec{\sigma}) \ldots \delta x_{s}^{\mu_{n}}(\vec{\sigma}) T_{\phi}^{\mu \nu}\left[x_{s}^{\beta}\left(T_{s}\right)+\epsilon_{u}^{\beta}\left(u\left(p_{s}\right)\right) \sigma^{u}\right]= \\
& =\epsilon_{r_{1}}^{\mu_{1}}\left(u\left(p_{s}\right)\right) \ldots \epsilon_{r_{n}}^{\mu_{n}}\left(u\left(p_{s}\right)\right) \epsilon_{A}^{\mu}\left(u\left(p_{s}\right)\right) \epsilon_{B}^{\nu}\left(u\left(p_{s}\right)\right) I_{T *}^{r_{1} . r_{n} A B}\left(T_{s}\right),
\end{aligned}
$$

and, again with the methods of Section $\mathrm{V}$, we can define the Dixon multipoles $\left[t_{\phi}^{\mu_{1} \ldots \mu_{n} \mu \nu}\left(T_{s}\right)\right.$ and the related ones] of the complex Klein-Gordon field in absence of interaction. In particular, from Eq. (5.10) we get $\tilde{t}_{\phi}^{\mu_{1}}\left(T_{s}\right)=\epsilon_{r_{1}}^{\mu_{1}}\left(u\left(p_{s}\right)\right) I_{T *}^{r_{1} \tau \tau}\left(T_{s}\right)$ with $I_{T *}^{r \tau \tau}\left(T_{s}\right) \equiv-P_{\phi}^{\tau} r_{\phi}^{r}=$ $\int d^{3} \sigma \sigma^{r}\left[\pi_{\phi^{*}} \pi_{\phi}+\vec{\partial} \phi^{*} \cdot \vec{\partial} \phi+m^{2} \phi^{*} \phi\right]\left(T_{s}, \vec{\sigma}\right)$ and its vanishing identifies Dixon's center of mass (Möller noncanonical center of energy) $\vec{r}_{\phi}$; then the canonical 3 -center of mass $\vec{q}_{\phi}$ can be obtained with the methods of Setion VI.

Then, as in the case of a real Klein-Gordon field, one can look for the canonical transformation from the center-of-phase canonical basis $X_{\phi}^{A}, P_{\phi}^{A}, R_{\phi}^{A}, Q_{\phi}^{A}, \mathbf{H}_{a}(\tau, \vec{k}), \mathbf{K}_{a}(\tau, \vec{k})$, to a center-of-mass canonical basis $q_{\phi}^{\tau}, P_{\phi}^{\tau^{\prime}}=\sqrt{\left(P_{\phi}^{\tau}\right)^{2}-\vec{P}_{\phi}^{2}}, \vec{q}_{\phi}, \vec{P}_{\phi}, R_{\phi}^{A^{\prime}}, Q_{\phi}^{A^{\prime}}, \mathbf{H}_{a}^{\prime}(\tau, \vec{k}), \mathbf{K}_{a}^{\prime}(\tau, \vec{k})$.

Now $\vec{R}_{\phi}^{\prime}$ shouls describe the relative position of the "centers of mass" for the " $a$ " and " $b$ " modes of the field configuration. In the free case Eqs.(D6) show that the theory can be reformulated in a non-local way as the sum of two theories, one for each mode: see Ref. 25] for the Lagrangian associated to Eqs.(D6) in the framework of pseudo-differential operators. This implies that the energy momentum tensor can be written as the sum of two pieces, one for each mode, and that the multipole expansion and the associated definition of center of mass can be applied to each piece. Already in the present framework, by using Eqs.(D5) and by making integrations by parts, we get $I_{T *}^{\tau \tau}\left(T_{s}\right)=\int d^{3} \sigma\left[\pi_{\phi^{*}} \pi_{\phi}+\vec{\partial} \phi^{*} \cdot \vec{\partial} \phi+\right.$ 
$\left.m^{2} \phi^{*} \phi\right]\left(T_{s}, \vec{\sigma}\right)=m \int d^{3} \sigma\left[\phi_{a}^{*} \sqrt{m^{2}+\triangle} \phi_{a}+\phi_{b}^{*} \sqrt{m^{2}+\triangle} \phi_{b}\right]\left(T_{s}, \vec{\sigma}\right)=I_{a *}^{\tau \tau}\left(T_{s}\right)+I_{b *}^{\tau \tau}\left(T_{s}\right)$ and $I_{T *}^{r \tau \tau}\left(T_{s}\right)=\int d^{3} \sigma\left[\pi_{\phi^{*}} \partial^{r} \phi+\pi_{\phi} \partial^{r} \phi\right]\left(T_{s}, \vec{\sigma}\right)=i m \int d^{3} \sigma\left[\phi_{a}^{*} \partial^{r} \phi_{a}+\phi_{b}^{*} \partial^{r} \phi_{b}\right]\left(T_{s}, \vec{\sigma}\right)=I_{a *}^{r \tau \tau}\left(T_{s}\right)+$ $I_{b *}^{r \tau \tau}\left(T_{s}\right) \equiv-P_{a \phi}^{\tau} r_{a \phi}^{r}-P_{b \phi}^{\tau} r_{b \phi}^{r}$. Then we could evaluate $\vec{q}_{a \phi}$ and $\vec{q}_{b \phi}$ and we expect to have $\vec{q}_{a \phi}=\vec{X}_{a \phi}^{\prime}=\vec{q}_{\phi}+\frac{1}{2} \vec{R}_{\phi}^{\prime}$ and $\vec{q}_{b \phi}=\vec{X}_{b \phi}^{\prime}=\vec{q}_{\phi}-\frac{1}{2} \vec{R}_{\phi}^{\prime}$.

The Dixon multipoles [9] for the electromagnetic current $\hat{J}_{\phi}^{\mu}$ are

$$
\begin{aligned}
& n \geq 0 \text {, } \\
& \hat{j}_{\phi}^{\mu_{1} \ldots \mu_{n} \mu}\left(T_{s}\right)=\hat{j}_{\phi}^{\left(\mu_{1} \ldots \mu_{n}\right) \mu}\left(T_{s}\right)=\int d^{3} \sigma \delta x_{s}^{\mu_{1}}(\vec{\sigma}) \ldots \delta x_{s}^{\mu_{n}}(\vec{\sigma}) \hat{J}_{\phi}^{\mu}\left(T_{s}, \vec{\sigma}\right)= \\
& =\epsilon_{r_{1}}^{\mu_{1}}\left(u\left(p_{s}\right)\right) \ldots \epsilon_{r_{n}}^{\mu_{n}}\left(u\left(p_{s}\right)\right) \\
& {\left[u^{\mu}\left(p_{s}\right) \int d^{3} \sigma \sigma^{r_{1}} \ldots \sigma^{r_{n}} i\left[\hat{\pi}_{\phi^{*}} \hat{\phi}^{*}-\hat{\pi}_{\phi} \hat{\phi}\right]\left(T_{s}, \vec{\sigma}\right)+\right.} \\
& \left.+\epsilon_{r}^{\mu}\left(u\left(p_{s}\right)\right) \int d^{3} \sigma \sigma^{r_{1}} \ldots \sigma^{r_{n}} i\left[\hat{\phi}^{*}\left(\partial^{r}-i e A_{\perp}^{r}\right) \hat{\phi}-\left(\partial^{r}+i e A_{\perp}^{r}\right) \hat{\phi}^{*} \hat{\phi}\right]\left(T_{s}, \vec{\sigma}\right)\right] \text {, } \\
& u_{\mu_{1}}\left(p_{s}\right) \hat{j}_{\phi}^{\mu_{1} \ldots \mu_{n} \mu}\left(T_{s}\right)=0, \\
& n=0 \text {, } \\
& \hat{j}_{\phi}^{\mu}\left(T_{s}\right)=q_{\phi} u^{\mu}\left(p_{s}\right)+\epsilon_{r}^{\mu}\left(u\left(p_{s}\right)\right) \\
& \int d^{3} \sigma i\left[\hat{\phi}^{*}\left(\partial^{r}-i e A_{\perp}^{r}\right) \hat{\phi}-\left(\partial^{r}+i e A_{\perp}^{r}\right) \hat{\phi}^{*} \hat{\phi}\right]\left(T_{s}, \vec{\sigma}\right)= \\
& =q_{\phi} u^{\mu}\left(p_{s}\right)+\epsilon_{r}^{\mu}\left(u\left(p_{s}\right)\right) i m \int d^{3} \sigma\left(\left(\hat{\phi}_{a}^{*}+\hat{\phi}_{b}\right) \frac{\partial^{r}}{\triangle}\left(\hat{\phi}_{a}+\hat{\phi}_{b}^{*}\right)-\right. \\
& \left.-i e A_{\perp}^{r}\left[\left(m^{2}+\triangle\right)^{-1 / 4}\left(\hat{\phi}_{a}^{*}+\hat{\phi}_{b}\right)\right]\left[\left(m^{2}+\triangle\right)^{-1 / 4}\left(\hat{\phi}_{a}+\hat{\phi}_{b}^{*}\right)\right]\right)\left(T_{s}, \vec{\sigma}\right), \\
& \hat{q}_{\phi}^{\mu_{1} \ldots \mu_{n}}\left(T_{s}\right)=\hat{q}_{\phi}^{\left(\mu_{1} \ldots \mu_{n}\right)}\left(T_{s}\right)=\hat{j}_{\phi}^{\mu_{1} \ldots \mu_{n} \mu}\left(T_{s}\right) u_{\mu}\left(p_{s}\right)=\quad(n>0) \\
& =\epsilon_{r_{1}}^{\mu_{1}}\left(u\left(p_{s}\right)\right) \ldots \epsilon_{r_{n}}^{\mu_{n}}\left(u\left(p_{s}\right)\right) \int d^{3} \sigma \sigma^{r_{1}} \ldots \sigma^{r_{n}} i\left[\hat{\pi}_{\phi^{*}} \hat{\phi}^{*}-\hat{\pi}_{\phi} \hat{\phi}\right]\left(T_{s}, \vec{\sigma}\right), \\
& u_{\mu_{1}}\left(p_{s}\right) \hat{q}_{\phi}^{\mu_{1} \ldots \mu_{n}}\left(T_{s}\right)=0 \text {. }
\end{aligned}
$$

In Ref. [9] it is shown how to arrive to the following reconstruction of the electromagnetic current in terms of the multipoles (if $f(k)$ is analytic)

$$
\begin{aligned}
& <\hat{J}_{\phi}^{\mu}, f>=\int d^{4} x \hat{J}_{\phi}^{\mu}(x) f(x)=\int d T_{s} \int \frac{d^{4} k}{(2 \pi)^{4}} \tilde{f}(k) e^{-i k \cdot x_{s}\left(T_{s}\right)} \\
& \int d^{3} \sigma \hat{J}_{\phi}^{\mu}\left[x_{s}\left(T_{s}\right)+\delta x_{s}(\vec{\sigma})\right] \sum_{n=0}^{\infty} \frac{(-i)^{n}}{n !} k_{\mu_{1}} \ldots k_{\mu_{n}} \hat{j}_{\phi}^{\mu_{1} \ldots \mu_{n} \mu}\left(T_{s}\right)= \\
& =\left.\int d T_{s} \sum_{n=0}^{\infty} \frac{1}{n !} \hat{j}_{\phi}^{\mu_{1} \ldots \mu_{n} \mu}\left(T_{s}\right) \frac{\partial^{n} f(x)}{\partial x_{s}^{\mu_{1}} \ldots \partial x_{s}^{\mu_{n}}}\right|_{x=x_{s}\left(T_{s}\right)}, \\
& \hat{J}_{\phi}^{\mu}(x)=\sum_{n=0}^{\infty} \frac{(-)^{n}}{n !} \frac{\partial^{n}}{\partial x_{s}^{\mu_{1}} \ldots \partial x_{s}^{\mu_{n}}} \int d T_{s} \delta^{4}\left(x-x_{s}\left(T_{s}\right)\right) \hat{j}_{\phi}^{\mu_{1} \ldots \mu_{n} \mu}\left(T_{s}\right) .
\end{aligned}
$$

It is also shown that if the multipoles $\hat{j}_{\phi}^{\mu_{1} \ldots \mu_{n} \mu}\left(T_{s}\right)$ are known for all $n>N$ for some fixed $\mathrm{N}$, then $\hat{J}_{\phi}^{\mu}$ and the multipoles with $n \leq N$ are completely determined.

See Appendix $\mathrm{C}$ for other types of multipoles. 


\section{CONCLUSIONS.}

In this paper we have made a detailed study of the kinematical description of scalar KleinGordon fields on the Wigner hyperplanes of the rest-frame Wigner-covariant instant form of dynamics. We have considered a Klein-Gordon field configuration as a relativistic extended object and we utilized both phase space techniques from relativistic particle mechanics and multipolar expansions from relativistic fluidodynamics to study aspects of the Klein-Gordon field which are usually ignored notwithstanding the relevance of scalar fields in physics: Higgs particles, Bose-Einstein condensation, Brans-Dicke scalar-tensor general relativity and multipolar expansions for the theory of gravitational waves, boson stars.

Simultaneously, we have used the Klein-Gordon field as an example to explore the description of isolated systems on the Wigner hyperplanes showing how the elusive concept of "relativistic center of mass" has to be divided in an "external" part (with respect to an arbitrary Lorentz frame) and in an "internal" part (inside the Wigner hyperplane). In both cases a canonical noncovariant Newton-Wigner-like center of mass, a covariant noncanonical Fokker-Pryce center of inertia and a noncanonical noncovariant Möller center of energy may be defined only in terms of the generators of suitable realizations of the Poincaé algebra. The three "internal" centers weakly coincide due to the three first class constraints defining the rest frame of the isolated system and are, therefore, gauge variables inside the Wigner hyperplanes. Namely there is the gauge freedom in the choice of the "external" timelike worldline to which they have to be attached. Now, in the description of the Wigner hyperplane with respect to an arbitrary Lorentz frame in Minkowski spacetime this gauge freedom is reflected in the arbitrariness of the choice of a timelike worldline $x_{s}^{\mu}(\tau)$, with noncanonical 4-coordinates, to be used as origin of the "internal" 3-coordinates. The natural gauge fixing for this gauge freedom is to put the three weakly coinciding "internal" centers in this origin: in this way the origin $x_{s}^{\mu}(\tau)$ is forced to coincide with the "external" covariant noncanonical Fokker-Pryce center of inertia and simultaneously to satisfy the conditions for being both the Pirani and the Tulczyjew centroid. Around this worldline there is a noncovariance worldtube (whose finite extension is measured by the Möller radius [22,24) containing all the pseudoworldlines of the noncovariant "external" canonical Newton-Wigner-like center of mass and noncanonical Möller center of energy.

Naturally, one could fix the gauge freedom in a different way by identifying a different "internal" collective 3 -vector with the origin $x_{s}^{\mu}$ : in such a case $x_{s}^{\mu}$ becomes one of the many possible covariant noncanonical centroids existing in literature and does not coincide with the "external" Fokker-Pryce center of inertia. For instance this happens for the Klein-Gordon field, because we do not yet know its canonical basis containing the "internal" center of mass 3-vector. In the Longhi-Materassi canonical decomposition of the Klein-Gordon field in collective and relative variables this 3-vector is replaced by another 3-vector, which can be named the "internal" center of phase of the field configuration by its construction.

The other main result of this paper is the identification of the canonical basis containing the "internal" center of phase of the Klein-Gordon field and of its multipoles in the framework of the rest-frame Wigner-covariant instant form of dynamics.

Therefore, many unrelated kinematical concepts find a well defined setting on the Wigner hyperplanes of the rest-frame Wigner-covariant instant form of dynamics, which seems to be the natural tool (like the separation of the center-of-mass motion in the nonrelativistic 
case) to be used in relativistic statistical mechanics and in lattice gauge theories due to its intrinsic Euclidean signature (without any Wick rotation) and to its associated description of the evolution by means of the Lorentz scalar rest-frame time measured by the clock of the decoupled (but noncovariant) point particle observer defined by the "external" canonical center of mass.

Even if much work is still needed to clarify all the aspects of the multipolar expansions of the Klein-Gordon field and of its properties as a relativistic extended object, especially for the charged fields of scalar electrodynamics, now we have a well defined framework in which to make further investigations and a first completely worked out canonical decomposition of the field in collective and relative variables.

A final comment on quantization. The canonical basis of the Klein-Gordon field containing the center of phase does not seem a good candidate for the quantization of the field in the rest-frame instant form of dynamics both due to the complicated expression of the original fields in terms of the new variables and to the fact that there is no sound canonical quantization of phases and angles (different is the case with their exponentials) as can be seen from the various articles contained in Ref. [23]. Let us remark that the problem of the non measurability of absolute phases (see the previous reference) is connected with the open problem of the measurability of the "external" decoupled noncovariant canonical center of mass of an isolated system (the quantization of these degrees of freedom would generate an equivalent of the "wave function of the universe") as it is clear from the Longhi-Materassi canonical basis, in which the collective position variable is built starting from the phases of the Fourier coefficients of the Klein-Gordon field. 


\section{APPENDIX A: NOTATIONS FOR SPACELIKE HYPERSURFACES.}

In this Appendix we will review the background material from Ref. [1] needed in the description of physical systems on spacelike hypersurfaces.

Let $\left\{\Sigma_{\tau}\right\}$ be a one-parameter family of spacelike hypersurfaces foliating Minkowski spacetime $M^{4}$ and giving a $3+1$ decomposition of it. At fixed $\tau$, let $z^{\mu}(\tau, \vec{\sigma})$ be the coordinates of the points on $\Sigma_{\tau}$ in $M^{4},\{\vec{\sigma}\}$ a system of coordinates on $\Sigma_{\tau}$. If $\sigma^{\check{A}}=\left(\sigma^{\tau}=\tau ; \vec{\sigma}=\left\{\sigma^{\check{r}}\right\}\right)$ [the notation $\check{A}=(\tau, \check{r})$ with $\check{r}=1,2,3$ will be used; note that $\check{A}=\tau$ and $\check{A}=\check{r}=1,2,3$ are Lorentz-scalar indices] and $\partial_{\check{A}}=\partial / \partial \sigma^{\check{A}}$, one can define the vierbeins

$$
z_{\check{A}}^{\mu}(\tau, \vec{\sigma})=\partial_{\check{A}} z^{\mu}(\tau, \vec{\sigma}), \quad \partial_{\check{B}} z_{\check{A}}^{\mu}-\partial_{\check{A}} z_{\check{B}}^{\mu}=0,
$$

so that the metric on $\Sigma_{\tau}$ is

$$
\begin{aligned}
& g_{\check{A} \check{B}}(\tau, \vec{\sigma})=z_{\check{A}}^{\mu}(\tau, \vec{\sigma}) \eta_{\mu \nu} z_{\breve{B}}^{\nu}(\tau, \vec{\sigma}), \quad g_{\tau \tau}(\tau, \vec{\sigma})>0, \\
& g(\tau, \vec{\sigma})=-\operatorname{det}\left\|g_{\check{A} \check{B}}(\tau, \vec{\sigma})\right\|=\left(\operatorname{det}\left\|z_{\check{A}}^{\mu}(\tau, \vec{\sigma})\right\|\right)^{2}, \\
& \gamma(\tau, \vec{\sigma})=-\operatorname{det}\left\|g_{\check{r} \check{s}}(\tau, \vec{\sigma})\right\| .
\end{aligned}
$$

If $\gamma^{\check{r} \check{s}}(\tau, \vec{\sigma})$ is the inverse of the 3-metric $g_{\check{r} \check{s}}(\tau, \vec{\sigma})\left[\gamma^{\check{r} \check{u}}(\tau, \vec{\sigma}) g_{\check{u} \check{s}}(\tau, \vec{\sigma})=\delta_{\breve{s}}^{\check{r}}\right]$, the inverse $g^{\check{A} \check{B}}(\tau, \vec{\sigma})$ of $g_{\check{A} \check{B}}(\tau, \vec{\sigma})\left[g^{\check{A} \check{C}}(\tau, \vec{\sigma}) g_{\check{c} \breve{b}}(\tau, \vec{\sigma})=\delta_{\breve{B}}^{\check{A}}\right]$ is given by

$$
\begin{aligned}
g^{\tau \tau}(\tau, \vec{\sigma}) & =\frac{\gamma(\tau, \vec{\sigma})}{g(\tau, \vec{\sigma})}, \\
g^{\tau \check{r}}(\tau, \vec{\sigma}) & =-\left[\frac{\gamma}{g} g_{\tau \check{u}} \gamma^{\check{r} \check{r}}\right](\tau, \vec{\sigma}), \\
g^{\check{r} \check{s}}(\tau, \vec{\sigma}) & =\gamma^{\check{r} \check{s}}(\tau, \vec{\sigma})+\left[\frac{\gamma}{g} g_{\tau \check{u}} g_{\tau \check{v}} \gamma^{\check{r} \check{r}} \gamma^{\check{v} \check{s}}\right](\tau, \vec{\sigma}),
\end{aligned}
$$

so that $1=g^{\tau \check{C}}(\tau, \vec{\sigma}) g_{\check{C} \tau}(\tau, \vec{\sigma})$ is equivalent to

$$
\frac{g(\tau, \vec{\sigma})}{\gamma(\tau, \vec{\sigma})}=g_{\tau \tau}(\tau, \vec{\sigma})-\gamma^{\check{r} \check{s}}(\tau, \vec{\sigma}) g_{\tau \check{r}}(\tau, \vec{\sigma}) g_{\tau \check{s}}(\tau, \vec{\sigma}) .
$$

We have

$$
z_{\tau}^{\mu}(\tau, \vec{\sigma})=\left(\sqrt{\frac{g}{\gamma}} l^{\mu}+g_{\tau \check{r}} \gamma^{\check{s} \breve{s}} z_{\breve{s}}^{\mu}\right)(\tau, \vec{\sigma}),
$$

and

$$
\begin{aligned}
\eta^{\mu \nu} & =z_{\tilde{A}}^{\mu}(\tau, \vec{\sigma}) g^{\check{A} \check{B}}(\tau, \vec{\sigma}) z_{\breve{B}}^{\nu}(\tau, \vec{\sigma})= \\
& =\left(l^{\mu} l^{\nu}+z_{\tilde{r}}^{\mu} \gamma^{\check{r} \check{s}} z_{\breve{s}}^{\nu}\right)(\tau, \vec{\sigma}),
\end{aligned}
$$

where

$$
\begin{aligned}
l^{\mu}(\tau, \vec{\sigma})= & \left(\frac{1}{\sqrt{\gamma}} \epsilon_{\alpha \beta \gamma}^{\mu} z_{1}^{\alpha} z_{2}^{\beta} z_{3}^{\gamma}\right)(\tau, \vec{\sigma}), \\
& l^{2}(\tau, \vec{\sigma})=1, \quad l_{\mu}(\tau, \vec{\sigma}) z_{\tilde{r}}^{\mu}(\tau, \vec{\sigma})=0,
\end{aligned}
$$


is the unit (future pointing) normal to $\Sigma_{\tau}$ at $z^{\mu}(\tau, \vec{\sigma})$.

For the volume element in Minkowski spacetime we have

$$
\begin{aligned}
d^{4} z & =z_{\tau}^{\mu}(\tau, \vec{\sigma}) d \tau d^{3} \Sigma_{\mu}=d \tau\left[z_{\tau}^{\mu}(\tau, \vec{\sigma}) l_{\mu}(\tau, \vec{\sigma})\right] \sqrt{\gamma(\tau, \vec{\sigma})} d^{3} \sigma= \\
& =\sqrt{g(\tau, \vec{\sigma})} d \tau d^{3} \sigma .
\end{aligned}
$$

Let us remark that according to the geometrical approach of Ref. [24], one can use Eq.(A5) in the form $z_{\tau}^{\mu}(\tau, \vec{\sigma})=N(\tau, \vec{\sigma}) l^{\mu}(\tau, \vec{\sigma})+N^{\check{r}}(\tau, \vec{\sigma}) z_{\check{r}}^{\mu}(\tau, \vec{\sigma})$, where $N=\sqrt{g / \gamma}=$ $\sqrt{g_{\tau \tau}-\gamma^{\check{r} \check{s}} g_{\tau \check{r}} g_{\tau \check{s}}}$ and $N^{\check{r}}=g_{\tau \check{s}} \gamma^{\check{s} \check{r}}$ are the standard lapse and shift functions, so that $g_{\tau \tau}=$ $N^{2}+g_{\check{r} \check{s}} N^{\check{r}} N^{\check{s}}, g_{\tau \check{r}}=g_{\check{r} \check{s}} N^{\check{s}}, g^{\tau \tau}=N^{-2}, g^{\tau \check{r}}=-N^{\check{r}} / N^{2}, g^{\check{r} \check{s}}=\gamma^{\check{r} \check{s}}+\frac{N^{\check{r}} N^{\check{s}}}{N^{2}}, \frac{\partial}{\partial z_{\tau}^{\mu}}=l_{\mu} \frac{\partial}{\partial N}+$ $z_{\check{s} \mu} \gamma^{\check{s} \check{r}} \frac{\partial}{\partial N^{\check{r}}}, d^{4} z=N \sqrt{\gamma} d \tau d^{3} \sigma$.

The rest frame form of a timelike fourvector $p^{\mu}$ is $\stackrel{\circ}{p}^{\mu}=\eta \sqrt{p^{2}}(1 ; \overrightarrow{0})=\eta^{\mu o} \eta \sqrt{p^{2}}, \stackrel{\circ}{p}^{2}=p^{2}$, where $\eta=\operatorname{sign} p^{o}$. The standard Wigner boost transforming $\stackrel{\circ}{p}^{\mu}$ into $p^{\mu}$ is

$$
\begin{aligned}
& L_{\nu}^{\mu}(p, \stackrel{\circ}{p})=\epsilon_{\nu}^{\mu}(u(p))= \\
&=\eta_{\nu}^{\mu}+2 \frac{p^{\mu} \stackrel{\circ}{p}_{\nu}}{p^{2}}-\frac{\left(p^{\mu}+\stackrel{\circ}{p}^{\mu}\right)\left(p_{\nu}+\stackrel{\circ}{p}_{\nu}\right)}{p \cdot \stackrel{\circ}{p}+p^{2}}= \\
&=\eta_{\nu}^{\mu}+2 u^{\mu}(p) u_{\nu}(\stackrel{\circ}{p})-\frac{\left(u^{\mu}(p)+u^{\mu}(\stackrel{\circ}{p})\right)\left(u_{\nu}(p)+u_{\nu}(\stackrel{\circ}{p})\right)}{1+u^{o}(p)} \\
& \nu=0 \quad \epsilon_{o}^{\mu}(u(p))=u^{\mu}(p)=p^{\mu} / \eta \sqrt{p^{2}} \\
& \nu=r \quad \epsilon_{r}^{\mu}(u(p))=\left(-u_{r}(p) ; \delta_{r}^{i}-\frac{u^{i}(p) u_{r}(p)}{1+u^{o}(p)}\right)
\end{aligned}
$$

The inverse of $L^{\mu}{ }_{\nu}(p, \stackrel{\circ}{p})$ is $L^{\mu}{ }_{\nu}(\stackrel{\circ}{p}, p)$, the standard boost to the rest frame, defined by

$$
L_{\nu}^{\mu}(\stackrel{\circ}{p}, p)=L_{\nu}{ }^{\mu}(p, \stackrel{\circ}{p})=\left.L_{\nu}^{\mu}(p, \stackrel{\circ}{p})\right|_{\vec{p} \rightarrow-\vec{p}}
$$

Therefore, we can define the following vierbeins [the $\epsilon_{r}^{\mu}(u(p))$ 's are also called polarization vectors; the indices $\mathrm{r}$, s will be used for $\mathrm{A}=1,2,3$ and $\bar{o}$ for $\mathrm{A}=0$ ]

$$
\begin{aligned}
& \epsilon_{A}^{\mu}(u(p))=L_{A}^{\mu}(p, \stackrel{\circ}{p}), \\
& \epsilon_{\mu}^{A}(u(p))=L_{\mu}^{A}(\stackrel{\circ}{p}, p)=\eta^{A B} \eta_{\mu \nu} \epsilon_{B}^{\nu}(u(p)), \\
& \epsilon_{\mu}^{\bar{o}}(u(p))=\eta_{\mu \nu} \epsilon_{o}^{\nu}(u(p))=u_{\mu}(p), \\
& \epsilon_{\mu}^{r}(u(p))=-\delta^{r s} \eta_{\mu \nu} \epsilon_{r}^{\nu}(u(p))=\left(\delta^{r s} u_{s}(p) ; \delta_{j}^{r}-\delta^{r s} \delta_{j h} \frac{u^{h}(p) u_{s}(p)}{1+u^{o}(p)}\right), \\
& \epsilon_{o}^{A}(u(p))=u_{A}(p)
\end{aligned}
$$

which satisfy 


$$
\begin{aligned}
& \epsilon_{\mu}^{A}(u(p)) \epsilon_{A}^{\nu}(u(p))=\eta_{\nu}^{\mu} \\
& \epsilon_{\mu}^{A}(u(p)) \epsilon_{B}^{\mu}(u(p))=\eta_{B}^{A} \\
& \eta^{\mu \nu}=\epsilon_{A}^{\mu}(u(p)) \eta^{A B} \epsilon_{B}^{\nu}(u(p))=u^{\mu}(p) u^{\nu}(p)-\sum_{r=1}^{3} \epsilon_{r}^{\mu}(u(p)) \epsilon_{r}^{\nu}(u(p)), \\
& \eta_{A B}=\epsilon_{A}^{\mu}(u(p)) \eta_{\mu \nu} \epsilon_{B}^{\nu}(u(p)) \\
& p_{\alpha} \frac{\partial}{\partial p_{\alpha}} \epsilon_{A}^{\mu}(u(p))=p_{\alpha} \frac{\partial}{\partial p_{\alpha}} \epsilon_{\mu}^{A}(u(p))=0 .
\end{aligned}
$$

The Wigner rotation corresponding to the Lorentz transformation $\Lambda$ is

$$
\begin{aligned}
R_{\nu}^{\mu}(\Lambda, p) & =\left[L(\stackrel{\circ}{p}, p) \Lambda^{-1} L(\Lambda p, \stackrel{\circ}{p})\right]_{\nu}^{\mu}=\left(\begin{array}{cc}
1 & 0 \\
0 & R^{i}{ }_{j}(\Lambda, p)
\end{array}\right), \\
R_{j}^{i}(\Lambda, p) & =\left(\Lambda^{-1}\right)^{i}{ }_{j}-\frac{\left(\Lambda^{-1}\right)^{i}{ }_{o} p_{\beta}\left(\Lambda^{-1}\right)^{\beta}{ }_{j}}{p_{\rho}\left(\Lambda^{-1}\right)_{o}{ }_{o}+\eta \sqrt{p^{2}}}- \\
& -\frac{p^{i}}{p^{o}+\eta \sqrt{p^{2}}}\left[\left(\Lambda^{-1}\right)^{o}{ }_{j}-\frac{\left(\left(\Lambda^{-1}\right)_{o}^{o}-1\right) p_{\beta}\left(\Lambda^{-1}\right)^{\beta}}{p_{\rho}\left(\Lambda^{-1}\right)^{\rho}{ }_{o}+\eta \sqrt{p^{2}}}\right] .
\end{aligned}
$$

The polarization vectors transform under the Poincaré transformations $(a, \Lambda)$ in the following way

$$
\epsilon_{r}^{\mu}(u(\Lambda p))=\left(R^{-1}\right)_{r}{ }^{s} \Lambda^{\mu}{ }_{\nu} \epsilon_{s}^{\nu}(u(p))
$$




\section{APPENDIX B: THE KLEIN-GORDON FIELD.}

Let us review the main results of Ref. [6]. For the sake of simplicity we shall denote $\phi(x)$ the Klein-Gordon field $\tilde{\phi}(x)$ of Section III.

Given a real field, solution of the Klein-Gordon equation, $\phi(x)=\phi^{*}(x)=\phi\left(x^{o}, \vec{x}\right)=$ $\int d \tilde{k}\left[a(\vec{k}) e^{-i k \cdot x}+a^{*}(\vec{k}) e^{i k \cdot x}\right]\left[\right.$ with $d \tilde{k}=d^{3} k / \Omega(k), \Omega(k)=(2 \pi)^{3} \omega(k), \omega(k)=k^{o}=\sqrt{m^{2}+k^{2}}$, $k=|\vec{k}| ; a(\vec{k})$ and $\partial a(\vec{k}) / \partial k^{i}$ are assumed to be in $L_{2}(d \tilde{k}) ; a(\vec{k})$ is $x^{o}$-independent in the free case without self interactions $V(\phi)]$, its conjugate momentum is $\pi(x)=\frac{\partial \phi(x)}{\partial x^{\circ}}=$ $-i \int d \tilde{k} \omega(k)\left[a(\vec{k}) e^{-i k \cdot x}-a^{*}(\vec{k}) e^{i k \cdot x}\right]$. The 10 Poincaré generators

$$
\begin{aligned}
P_{\phi}^{\mu}= & \frac{1}{2} \int d^{3} x\left[\pi \partial^{\mu} \phi-\frac{1}{2} \eta^{o \mu}\left[\pi^{2}-(\vec{\partial} \phi)^{2}-m^{2} \phi^{2}\right]\right]\left(x^{o}, \vec{x}\right)= \\
= & \int d \tilde{k} k^{\mu} a^{*}(\vec{k}) a(\vec{k}) \\
J_{\phi}^{\mu \nu}= & \int d^{3} x\left[\left(\pi\left(x^{\mu} \partial^{\nu}-x^{\nu} \partial^{\mu}\right) \phi\right)\left(x^{o}, \vec{x}\right)-\frac{1}{2}\left(x^{\mu} \eta^{\nu o}-x^{\nu} \eta^{\mu o}\right)\left(\pi^{2}-(\vec{\partial} \phi)^{2}-m^{2} \phi^{2}\right)\right]\left(x^{o}, \vec{x}\right), \\
J_{\phi}^{i j}= & \int d^{3} x\left[\pi\left(x^{i} \partial^{j}-x^{j} \partial^{i}\right) \phi\right]\left(x^{o}, \vec{x}\right)= \\
= & -i \int d \tilde{k} a^{*}(\vec{k})\left(k^{i} \frac{\partial}{\partial k^{j}}-k^{j} \frac{\partial}{\partial k^{i}}\right) a(\vec{k}) \\
J_{\phi}^{o i}= & x^{o} \int d^{3} x\left(\pi \partial^{i} \phi\right)\left(x^{o}, \vec{x}\right)-\frac{1}{2} \int d^{3} x x^{i}\left[\pi^{2}+(\vec{\partial} \phi)^{2}+m^{2} \phi^{2}\right]\left(x^{o}, \vec{x}\right)= \\
= & x^{o} P_{\phi}^{i}-i \int d \tilde{k} a^{*}(\vec{k}) \omega(k) \frac{\partial}{\partial k^{i}} a(\vec{k}) \\
& \left\{P_{\phi}^{\mu}, P_{\phi}^{\nu}\right\}=0, \quad\left\{J_{\phi}^{\mu \nu}, p_{\phi}^{\rho}\right\}=P_{\phi}^{\mu} \eta^{\nu \rho}-P_{\phi}^{\nu} \eta^{\mu \rho} \\
& \left\{J_{\phi}^{\mu \nu}, J_{\phi}^{\rho \sigma}\right\}=\eta^{\mu \sigma} J_{\phi}^{\nu \rho}+\eta^{\nu \rho} J_{\phi}^{\mu \sigma}-\eta^{\mu \rho} J_{\phi}^{\nu \sigma}-\eta^{\nu \sigma} J_{\phi}^{\mu \rho}
\end{aligned}
$$

are finite if $|a(\vec{k})| \rightarrow_{k>>m} k^{-\frac{3}{2}-\sigma}, \sigma>0$, and $|a(\vec{k})| \rightarrow_{k<<m} k^{-\frac{3}{2}+\epsilon}, \epsilon>0$.

By putting $a(\vec{k})=\sqrt{I(\vec{k})} e^{i \varphi(\vec{k})}$, one gets

$P_{\phi}^{\mu}=\int d \tilde{k} k^{\mu} I(\vec{k})$,

$J_{\phi}^{i j}=\int d \tilde{k} I(\vec{k})\left(k^{i} \frac{\partial}{\partial k^{j}}-k^{j} \frac{\partial}{\partial k^{i}}\right) \varphi(\vec{k})$,

$J_{\phi}^{o i}=x^{o} P_{\phi}^{i}+\int d \tilde{k} I(\vec{k}) \omega(k) \frac{\partial}{\partial k^{i}} \varphi(\vec{k})$,

and they are finite if: a) for $k \rightarrow \infty$ one has $\left.|I(\vec{k})| \rightarrow k^{-3-\sigma}, \sigma>0,|\varphi(\vec{k})| \rightarrow k ; \mathrm{b}\right)$ for $k \rightarrow 0$ one has $|I(\vec{k})| \rightarrow k^{-3+\epsilon}, \epsilon>0,|\varphi(\vec{k})| \rightarrow k^{\eta}, \eta>-\epsilon$.

The non vanishing Poisson brackets of these 3 canonical bases are: $\left\{\phi\left(x^{o}, \vec{x}\right), \pi\left(x^{o}, \vec{y}\right)\right\}=$ $\delta^{3}(\vec{x}-\vec{y}),\left\{a(\vec{k}), a^{*}(\vec{q})\right\}=-i \Omega(k) \delta^{3}(\vec{k}-\vec{q}),\{I(\vec{k}), \varphi(\vec{q})\}=\Omega(k) \delta^{3}(\vec{k}-\vec{q})$.

Let $F\left(P_{\phi}, k\right)=F\left(P_{\phi} \cdot k, P_{\phi}^{2}\right)$ be a real scalar weight function, arbitrary apart the normalization $\int d \tilde{k} k^{\mu} F\left(P_{\phi}, k\right)=P_{\phi}^{\mu}$; a simple choice for $F$ is $F \approx e^{-P_{\phi} \cdot k}$. One assumes a behaviour of $F$ for $k \rightarrow \infty$ and $k \rightarrow 0$ such that all the integrals, which depend on $F$, are finite.

Then one can define the following canonical basis 


$$
\begin{aligned}
X_{\phi}^{\mu}= & \int d \tilde{k} \varphi(\vec{k}) \frac{\partial}{\partial P_{\phi \mu}} F\left(P_{\phi}, k\right), \\
P_{\phi}^{\mu}= & \int d \tilde{k} k^{\mu} I(\vec{k})=\int d \tilde{k} k^{\mu} F\left(P_{\phi}, k\right), \\
\mathcal{H}(\vec{k})= & \int d \tilde{k}^{\prime} G\left(\vec{k}, \vec{k}^{\prime}\right)\left[I\left(\vec{k}^{\prime}\right)-F\left(P_{\phi}, k^{\prime}\right)\right] \\
& \rightarrow_{k \rightarrow \infty} k^{-3-\sigma}, \quad \sigma>0 ; \quad \rightarrow_{k \rightarrow 0} k^{-1+\epsilon}, \quad \epsilon>0, \\
\mathcal{K}(\vec{k})= & \mathcal{D} \varphi(\vec{k}) \rightarrow_{k \rightarrow \infty} k^{1-\epsilon}, \quad \epsilon>0 ; \quad \rightarrow_{k \rightarrow 0} k^{\eta-2}, \quad \eta>-\epsilon, \\
& \left\{X_{\phi}^{\mu}, P_{\phi}^{\nu}\right\}=-\eta^{\mu \nu}, \quad\{\mathcal{H}(\vec{k}), \mathcal{K}(\vec{q})\}=\Omega(k) \delta^{3}(\vec{k}-\vec{q}), \\
I(\vec{k})= & \mathcal{D H}(\vec{k})+F\left(P_{\phi}, k\right), \quad \\
\varphi(\vec{k})= & k \cdot X_{\phi}+\int d \tilde{k}^{\prime} \int d \tilde{k}^{\prime \prime} \mathcal{K}\left(\vec{k}^{\prime}\right) G\left(\vec{k}^{\prime}, \vec{k}^{\prime \prime}\right) \triangle(\vec{k}, \vec{k}) .
\end{aligned}
$$

Here, $\mathcal{D}$ is the non compact operator $\mathcal{D}=3-m^{2} \triangle_{L B}$, with $\triangle_{L B}$ being the LaplaceBeltrami operator on the mass shell submanifold $H_{3}^{1}$ defined by $k^{\mu} k_{\mu}=m^{2}$ and $k^{o}>0$. The Green function $G$ satisfies $\mathcal{D}_{\vec{k}} G(\vec{k}, \vec{q})=\Omega(k) \delta^{3}(\vec{k}-\vec{q})$. Finally, one has $\triangle(\vec{k}, \vec{q})=$ $\Omega(k) \delta^{3}(\vec{k}-\vec{q})-q^{\mu} \frac{\partial}{\partial P_{\phi}^{\mu}} F\left(P_{\phi}, k\right)$.

The Poincaré generators are given by

$$
\begin{aligned}
& P_{\phi}^{\mu}, \\
& J_{\phi}^{i j}=X_{\phi}^{i} P_{\phi}^{j}-X_{\phi}^{j} P_{\phi}^{i}+\int d \tilde{k} \mathcal{H}(\vec{k})\left(k^{i} \frac{\partial}{\partial k^{j}}-k^{j} \frac{\partial}{\partial k^{i}}\right) \mathcal{K}(\vec{k}), \\
& J_{\phi}^{o i}=\left(x^{o}+X_{\phi}^{o}\right) P_{\phi}^{i}-X_{\phi}^{i} P_{\phi}^{o}+\int d \tilde{k} \mathcal{H}(\vec{k}) \omega(k) \frac{\partial}{\partial k^{i}} \mathcal{K}(\vec{k}) .
\end{aligned}
$$

This canonical transformation exists (without problems from the existence of the zero modes of the operator $\mathcal{D}$ ) only if the field configurations $\phi(x)$, solution of the Klein-Gordon equation, with $\pi(x)=\partial \phi(x) / \partial x^{o}$, satisfy the conditions:

A) $P_{l m}=\int d \tilde{k} I(\vec{k}) v_{1,-3, l m}^{(o)}(\vec{k}) \equiv \int d \tilde{k} F\left(P_{\phi}, k\right) v_{1,-3, l m}^{(o)}(\vec{k})=0$, and, in the case that $I(\vec{k}) \rightarrow_{k \rightarrow 0} k^{-3+\eta}$ with $\eta>l+1$, also B) $Q_{l m}=\int d \tilde{k} I(\vec{k}) v_{2,-3, l m}^{(o)}(\vec{k}) \equiv \int d \tilde{k} F\left(P_{\phi}, k\right) v_{2,-3, l m}^{(o)}(\vec{k})=0$, where the $v^{(o)}$ 's are zero modes of $\mathcal{D}$. The conditions A) are non void only for $l \geq 2$ and are called "no supertranslations conditions" in Ref. [7]: when they are satisfied, the constants of motion $P_{l m}$ are functionally dependent on the momenta of the new canonical basis [when they are not satisfied, the $P_{l m}$ are independent constants of motion allowing the definition of a BMS algebra and this destroys the possibility of definining a unique Poincaré algebra in a non ambigous way].

One has

$$
\begin{aligned}
\phi(x)= & \int d \tilde{k} \sqrt{F\left(P_{\phi}, k\right)+\mathcal{D} \mathcal{H}(\vec{k})} \\
& {\left[e^{i k \cdot\left(X_{\phi}-x\right)+i \int d \tilde{k}^{\prime} \int d \tilde{k} " \mathcal{K}\left(\vec{k}^{\prime}\right) G\left(\vec{k}^{\prime}, \vec{k}^{\prime \prime}\right) \triangle(\vec{k}, \vec{k})}+c . c .\right], } \\
\pi(x)= & -i \int d \tilde{k} \omega(k) \sqrt{F\left(P_{\phi}, k\right)+\mathcal{D} \mathcal{H}(\vec{k})}
\end{aligned}
$$




$$
\left[e^{i k \cdot\left(X_{\phi}-x\right)+i \int d \tilde{k}^{\prime} \int d \tilde{k}^{\prime \prime} \mathcal{K}\left(\vec{k}^{\prime}\right) G\left(\vec{k}^{\prime}, \vec{k}^{\prime \prime}\right) \triangle\left(\vec{k}^{\prime}, \vec{k}\right)}-c . c .\right] .
$$

Let us remark that on the Wigner hyperplane $F\left(P_{\phi}, k\right)$ may be replaced with two scalar functions $\tilde{F}^{\tau}\left(P_{\phi}^{\tau}, q\right), \tilde{F}\left(\vec{P}_{\phi}, \vec{q}\right)\left[F \mapsto \tilde{\mathcal{F}}\left(P_{\phi}, q\right)=\tilde{F}^{\tau}\left(P_{\phi}^{\tau},|\vec{q}|\right)-\tilde{F}\left(\vec{P}_{\phi}, \vec{q}\right)\right]$, so that the centerof-mass variables would be

$$
\begin{aligned}
X_{\phi}^{\tau} & =\int d \tilde{q} \varphi(\tau, \vec{q}) \frac{\partial \tilde{F}^{\tau}\left(P_{\phi}^{\tau}, q\right)}{\partial P_{\phi}^{\tau}}, \\
P_{\phi}^{\tau} & =\int d \tilde{q} \omega(q) I(\tau, \vec{q})=\int d \tilde{q} \omega(q) \tilde{F}^{\tau}\left(P_{\phi}^{\tau}, q\right), \\
\vec{X}_{\phi} & =\int d \tilde{q} \varphi(\tau, \vec{q}) \frac{\partial \tilde{F}\left(\vec{P}_{\phi}, \vec{q}\right)}{\partial \vec{P}_{\phi}} \\
\vec{P}_{\phi} & =\int d \tilde{q} \vec{q} I(\tau, \vec{q})=\int d \tilde{q} \vec{q} \tilde{F}\left(\vec{P}_{\phi}, \vec{q}\right) .
\end{aligned}
$$

Instead of this generically non polynomial weight functions, we use in this paper weight functions linear in the Poincaré momenta: $\tilde{F}^{\tau}\left(P_{\phi}^{\tau}, q\right)=P_{\phi}^{\tau} F^{\tau}(q), \tilde{F}\left(\vec{P}_{\phi}, \vec{q}\right)=\vec{P}_{\phi} \cdot \vec{q} F(q)$. 


\section{APPENDIX C: MORE ON DIXON'S MULTIPOLES.}

Let us add other forms of the Dixon multipoles.

In the case of the real Klein-Gordon field treated in Section V, the Hamilton equations (4.40) imply [in Ref. [9] this is a consequence of $\partial_{\mu} T^{\mu \nu} \stackrel{\circ}{=} 0$ ]

$$
\begin{gathered}
\frac{d p_{T}^{\mu}\left(T_{s}\right)}{d T_{s}} \stackrel{\circ}{=} 0, \quad \text { for } n=0, \\
\frac{d p_{T}^{\mu_{1} \ldots \mu_{n} \mu}\left(T_{s}\right)}{d T_{s}} \stackrel{\circ}{=}-n u^{\left(\mu_{1}\right.}\left(p_{s}\right) p_{T}^{\left.\mu_{2} \ldots \mu_{n}\right) \mu}\left(T_{s}\right)+n t_{T}^{\left(\mu_{1} \ldots \mu_{n}\right) \mu}\left(T_{s}\right), \quad n \geq 1 .
\end{gathered}
$$

Let us define for $n \geq 1$

$$
\begin{aligned}
b_{T}^{\mu_{1} \ldots \mu_{n} \mu}\left(T_{s}\right)= & p_{T}^{\left(\mu_{1} \ldots \mu_{n} \mu\right)}\left(T_{s}\right)= \\
= & \epsilon_{r_{1}}^{\left(\mu_{1}\right.}\left(u\left(p_{s}\right)\right) \ldots \epsilon_{r_{n}}^{\mu_{n}}\left(u\left(p_{s}\right)\right) \epsilon_{A}^{\mu)}\left(u\left(p_{s}\right)\right) I_{T}^{r_{1} \ldots r_{n} A \tau}\left(T_{s}\right), \\
c_{T}^{\mu_{1} \ldots \mu_{n} \mu}\left(T_{s}\right)= & c_{T}^{\left(\mu_{1} \ldots \mu_{n}\right) \mu}\left(T_{s}\right)=p_{T}^{\mu_{1} \ldots \mu_{n} \mu}\left(T_{s}\right)-p_{T}^{\left(\mu_{1} \ldots \mu_{n} \mu\right)}\left(T_{s}\right)= \\
= & {\left[\epsilon_{r_{1}}^{\mu_{1}}\left(u\left(p_{s}\right)\right) \ldots \epsilon_{r_{n}}^{\mu_{n}} \epsilon_{A}^{\mu}\left(u\left(p_{s}\right)\right)-\epsilon_{r_{1}}^{\left(\mu_{1}\right.}\left(u\left(p_{s}\right)\right) \ldots \epsilon_{r_{n}}^{\mu_{n}}\left(u\left(p_{s}\right)\right) \epsilon_{A}^{\mu)}\left(u\left(p_{s}\right)\right)\right] I_{T}^{r_{1} \ldots r_{n} A \tau}\left(T_{s}\right), } \\
& c_{T}^{\mu_{1} \ldots \mu_{n} \mu}\left(T_{s}\right)=0,
\end{aligned}
$$

and then for $n \geq 2$

$$
\begin{aligned}
d_{T}^{\mu_{1} \ldots \mu_{n} \mu \nu}\left(T_{s}\right)= & d_{T}^{\left(\mu_{1} \ldots \mu_{n}\right)(\mu \nu)}\left(T_{s}\right)=t_{T}^{\mu_{1} \ldots \mu_{n} \mu \nu}\left(T_{s}\right)- \\
& -\frac{n+1}{n}\left[t_{T}^{\left(\mu_{1} \ldots \mu_{n} \mu\right) \nu}\left(T_{s}\right)+t_{T}^{\left(\mu_{1} \ldots \mu_{n} \nu\right) \mu}\left(T_{s}\right)\right]+\frac{n+2}{n} t_{T}^{\left(\mu_{1} \ldots \mu_{n} \mu \nu\right)}\left(T_{s}\right)= \\
= & {\left[\epsilon_{r_{1} \ldots}^{\mu_{1}} \ldots \epsilon_{r_{n}}^{\mu_{n}} \epsilon_{A}^{\mu} \epsilon_{B}^{\nu}-\frac{n+1}{n}\left(\epsilon_{r_{1}}^{\left(\mu_{1} \ldots\right.} \epsilon_{r_{n}}^{\mu_{n}} \epsilon_{A}^{\mu)} \epsilon_{B}^{\nu}+\right.\right.} \\
+ & \left.\left.\epsilon_{r_{1}}^{\left(\mu_{1} \ldots\right.} \epsilon_{r_{n}}^{\mu_{n}} \epsilon_{B}^{\nu)} \epsilon_{A}^{\mu}\right)+\frac{n+2}{n} \epsilon_{r_{1}}^{\left(\mu_{1}\right.} . . \epsilon_{r_{n}}^{\mu_{n}} \epsilon_{A}^{\mu} \epsilon_{B}^{\nu}\right]\left(u\left(p_{s}\right)\right) I_{T}^{r_{1} . . r_{n} A B}\left(T_{s}\right), \\
& d_{T}^{\left(\mu_{1} \ldots \mu_{n} \mu\right) \nu}\left(T_{s}\right)=0 .
\end{aligned}
$$

Then Eqs.(C1) may be rewritten in the form

$$
\begin{aligned}
1) & n=1 \\
t_{T}^{\mu \nu}\left(T_{s}\right)= & t_{T}^{(\mu \nu)}\left(T_{s}\right) \stackrel{\circ}{=} p_{T}^{\mu}\left(T_{s}\right) u^{\nu}\left(p_{s}\right)+\frac{1}{2} \frac{d}{d T_{s}}\left(S_{T}^{\mu \nu}\left(T_{s}\right)[\phi]+2 b_{T}^{\mu \nu}\left(T_{s}\right)\right), \\
& \Downarrow \\
t_{T}^{\mu \nu}\left(T_{s}\right) \stackrel{\circ}{=} & p_{T}^{(\mu}\left(T_{s}\right) u^{\nu)}\left(p_{s}\right)+\frac{d}{d T_{s}} b_{T}^{\mu \nu}\left(T_{s}\right)=P_{\phi}^{\tau} u^{\mu}\left(p_{s}\right) u^{\nu}\left(p_{s}\right)+P_{\phi}^{r} u^{(\mu}\left(p_{s}\right) \epsilon_{r}^{\nu)}\left(u\left(p_{s}\right)\right)+ \\
+ & \epsilon_{r}^{(\mu}\left(u\left(p_{s}\right)\right) u^{\nu)}\left(p_{s}\right) \int d^{3} \sigma \sigma^{r} \frac{1}{2}\left[\pi^{2}+(\vec{\partial} \phi)^{2}+m^{2} \phi^{2}\right]\left(T_{s}, \vec{\sigma}\right)+ \\
+ & \epsilon_{r}^{(\mu}\left(u\left(p_{s}\right)\right) \epsilon_{s}^{\nu)}\left(u\left(p_{s}\right)\right) \int d^{3} \sigma \sigma^{r}\left[\pi \partial^{s} \phi\right]\left(T_{s}, \vec{\sigma}\right),
\end{aligned}
$$




$$
\begin{aligned}
& \frac{d}{d T_{s}} S_{T}^{\mu \nu}\left(T_{s}\right)[\phi] \stackrel{\circ}{=} 2 p_{T}^{[\mu}\left(T_{s}\right) u^{\nu]}\left(p_{s}\right)=2 P_{\phi}^{r} \epsilon_{r}^{[\mu}\left(u\left(p_{s}\right)\right) u^{\nu]}\left(p_{s}\right) \approx 0 \\
&2) \quad n=2 \quad\left[\text { identity } t_{T}^{\rho \mu \nu}=t_{T}^{(\rho \mu) \nu}+t_{T}^{(\rho \nu) \mu}+t_{T}^{(\mu \nu) \rho}\right] \\
& 2 t_{T}^{(\rho \mu) \nu}\left(T_{s}\right) \stackrel{\circ}{=} 2 u^{(\rho}\left(p_{s}\right) b_{T}^{\mu) \nu}\left(T_{s}\right)+u^{(\rho}\left(p_{s}\right) S_{T}^{\mu) \nu}\left(T_{s}\right)[\phi]+\frac{d}{d T_{s}}\left(b_{T}^{\rho \mu \nu}\left(T_{s}\right)+c_{T}^{\rho \mu \nu}\left(T_{s}\right)\right), \\
& \Downarrow \\
& t_{T}^{\rho \mu \nu}\left(T_{s}\right) \stackrel{\circ}{=} u^{\rho}\left(p_{s}\right) b_{T}^{\mu \nu}\left(T_{s}\right)+S_{T}^{\rho(\mu}\left(T_{s}\right)[\phi] u^{\nu)}\left(p_{s}\right)+\frac{d}{d T_{s}}\left(\frac{1}{2} b_{T}^{\rho \mu \nu}\left(T_{s}\right)-c_{T}^{\rho \mu \nu}\left(T_{s}\right)\right), \\
&\quad 3) \quad n \geq 3 \\
& t_{T}^{\mu_{1} \ldots \mu_{n} \mu \nu}\left(T_{s}\right) \stackrel{\circ}{=} d_{T}^{\mu_{1} \ldots \mu_{n} \mu \nu}\left(T_{s}\right)+u^{\left(\mu_{1}\right.}\left(p_{s}\right) b_{T}^{\left.\mu_{2} \ldots \mu_{n}\right) \mu \nu}\left(T_{s}\right)+2 u^{\left(\mu_{1}\right.}\left(p_{s}\right) c_{T}^{\left.\mu_{2} \ldots \mu_{n}\right)(\mu \nu)}\left(T_{s}\right)+ \\
&= \frac{2}{n} c_{T}^{\mu_{1} \ldots \mu_{n}(\mu}\left(T_{s}\right) u^{\nu)}\left(p_{s}\right)+\frac{d}{d T_{s}}\left[\frac{1}{n+1} b_{T}^{\mu_{1} \ldots \mu_{n} \mu \nu}\left(T_{s}\right)+\frac{2}{n} c_{T}^{\mu_{1} \ldots \mu_{n}(\mu \nu)}\left(T_{s}\right)\right],
\end{aligned}
$$

This allows [9] to rewrite $\left\langle T^{\mu \nu}, f\right\rangle$ in the following form

$$
\begin{aligned}
<T^{\mu \nu}, f> & =\int d T_{s} \int \frac{d^{4} k}{(2 \pi)^{4}} \tilde{f}(k) e^{-i k \cdot x_{s}\left(T_{s}\right)}\left[u^{(\mu}\left(p_{s}\right) p_{T}^{\nu)}\left(T_{s}\right)-i k_{\rho} S_{T}^{\rho(\mu}\left(T_{s}\right)[\phi] u^{\nu)}\left(p_{s}\right)+\right. \\
& \left.+\sum_{n=2}^{\infty} \frac{(-i)^{n}}{n !} k_{\rho_{1}} \ldots k_{\rho_{n}} \mathcal{I}_{T}^{\rho_{1} \ldots \rho_{n} \mu \nu}\left(T_{s}\right)\right]
\end{aligned}
$$

with

$$
\begin{aligned}
\mathcal{I}_{T}^{\mu_{1} \ldots \mu_{n} \mu \nu}\left(T_{s}\right)= & \mathcal{I}_{T}^{\left(\mu_{1} \ldots \mu_{n}\right)(\mu \nu)}\left(T_{s}\right)=d_{T}^{\mu_{1} \ldots \mu_{n} \mu \nu}\left(T_{s}\right)- \\
- & \frac{2}{n-1} u^{\left(\mu_{1}\right.}\left(p_{s}\right) c_{T}^{\left.\mu_{2} \ldots \mu_{n}\right)(\mu \nu)}\left(T_{s}\right)+\frac{2}{n} c_{T}^{\mu_{1} \ldots \mu_{n}(\mu}\left(T_{s}\right) u^{\nu)}\left(p_{s}\right)= \\
= & {\left[\epsilon_{r_{1}}^{\mu_{1}} \ldots \epsilon_{r_{n}}^{\mu_{n}} \epsilon_{A}^{\mu} \epsilon_{B}^{\nu}-\frac{n+1}{n}\left(\epsilon_{r_{1}}^{\left(\mu_{1}\right.} \ldots \epsilon_{r_{n}}^{\mu_{n}} \epsilon_{A}^{\mu)} \epsilon_{B}^{\nu}+\right.\right.} \\
+ & \left.\left.\epsilon_{r_{1}}^{\left(\mu_{1} \ldots\right.} \epsilon_{r_{n}}^{\mu_{n}} \epsilon_{B}^{\nu)} \epsilon_{A}^{\mu}\right)+\frac{n+2}{n} \epsilon_{r_{1}}^{\left(\mu_{1}\right.} \ldots \epsilon_{r_{n}}^{\mu_{n}} \epsilon_{A}^{\mu} \epsilon_{B}^{\nu)}\right]\left(u\left(p_{s}\right) 0 I_{T}^{r_{1} . . r_{n} A B}\left(T_{s}\right)-\right. \\
- & {\left[\frac{2}{n-1} u^{\left(\mu_{1}\right.}\left(p_{s}\right)\left(\epsilon_{r_{1}}^{\mu_{2} \ldots} \epsilon_{r_{n-1}}^{\left.\mu_{n}\right)} \epsilon_{r_{n}}^{(\mu} \epsilon_{A}^{\nu)}-\epsilon_{r_{1}}^{\left(\mu_{2} \ldots\right.} \epsilon_{r_{n-1}}^{\left.\mu_{n}\right)} \epsilon_{r_{n}}^{(\mu} \epsilon_{A}^{\nu))}\right)-\right.} \\
- & \frac{2}{n}\left(\epsilon_{r_{1}}^{\mu_{1}} \ldots \epsilon_{r_{n}}^{\mu_{n}} \epsilon_{A}^{(\mu}-\epsilon_{r_{1}}^{\left(\mu_{1} \ldots\right.} \epsilon_{r_{n}}^{\mu_{n}} \epsilon_{A}^{(\mu)} u^{\nu)}\left(p_{s}\right)\right]\left(u\left(p_{s}\right) 0 I_{T}^{r_{1} . . r_{n} A \tau}\left(T_{s}\right),\right. \\
& \mathcal{I}_{T}^{\left(\mu_{1} \ldots \mu_{n} \mu\right) \nu}\left(T_{s}\right)=0 .
\end{aligned}
$$

Finally, a set of multipoles equivalent to the $\mathcal{I}_{T}^{\mu_{1} \ldots \mu_{n} \mu \nu}$ is

$$
\begin{gathered}
n \geq 0 \\
J_{T}^{\mu_{1} \ldots \mu_{n} \mu \nu \rho \sigma}\left(T_{s}\right)=J_{T}^{\left(\mu_{1} \ldots \mu_{n}\right)[\mu \nu][\rho \sigma]}\left(T_{s}\right)=\mathcal{I}_{T}^{\mu_{1} \ldots \mu_{n}[\mu[\rho \nu] \sigma]}\left(T_{s}\right)= \\
=t_{T}^{\mu_{1} \ldots \mu_{n}[\mu[\rho \nu] \sigma]}\left(T_{s}\right)-\frac{1}{n+1}\left[u^{[\mu}\left(p_{s}\right) p_{T}^{\nu] \mu_{1} \ldots \mu_{n}[\rho \sigma]}\left(T_{s}\right)+\right.
\end{gathered}
$$




$$
\begin{aligned}
+ & \left.u^{[\rho}\left(p_{s}\right) p_{T}^{\sigma] \mu_{1} \ldots \mu_{n}[\mu \nu]}\left(T_{s}\right)\right]= \\
= & {\left[\epsilon_{r_{1}}^{\mu_{1}} . . \epsilon_{r_{n}}^{\mu_{n}} \epsilon_{r}^{[\mu} \epsilon_{s}^{[\rho} \epsilon_{A}^{\nu]} \epsilon_{B}^{\sigma]}\right]\left(u\left(p_{s}\right)\right) I_{T}^{r_{1} . . r_{n} A B}\left(T_{s}\right)-} \\
- & \frac{1}{n+1}\left[u^{[\mu}\left(p_{s}\right) \epsilon_{r}^{\nu]}\left(u\left(p_{s}\right)\right) \epsilon_{s}^{[\rho}\left(u\left(p_{s}\right)\right) \epsilon_{A}^{\sigma]}\left(u\left(p_{s}\right)\right)+\right. \\
+ & \left.u^{[\rho}\left(p_{s}\right) \epsilon_{r}^{\sigma]}\left(u\left(p_{s}\right)\right) \epsilon_{s}^{[\mu}\left(u\left(p_{s}\right)\right) \epsilon_{A}^{\nu]}\left(u\left(p_{s}\right)\right)\right] \\
& \epsilon_{r_{1}}^{\mu_{1}}\left(u\left(p_{s}\right)\right) \ldots \epsilon_{r_{n}}^{\mu_{n}}\left(u\left(p_{s}\right)\right) I_{T}^{r r_{1} . . r_{n} s A \tau}\left(T_{s}\right), \\
& {[(n+4)(3 n+5) \text { linearly independent components]},} \\
& n \geq 1 \\
& J_{T}^{\mu_{1} \ldots \mu_{n} \mu \nu \rho \sigma}\left(T_{s}\right)=J_{T}^{\mu_{1} \ldots \mu_{n-1}\left(\mu_{n} \mu \nu\right) \rho \sigma}\left(T_{s}\right)=0, \\
u_{\mu_{1}}\left(p_{s}\right) & n \geq 2 \\
\mathcal{I}_{T}^{\mu_{1} \ldots \mu_{n} \mu \nu}\left(T_{s}\right)= & \frac{4(n-1)}{n+1} J_{T}^{\left(\mu_{1} \ldots \mu_{n-1}|\mu| \mu_{n}\right) \nu}\left(T_{s}\right) .
\end{aligned}
$$

The $J_{T}^{\mu_{1} \ldots \mu_{n} \mu \nu \rho \sigma}$ are the Dixon " $2^{n+2}$-pole inertial moment tensors" of the extended system: they [or equivalently the $\mathcal{I}_{T}^{\mu_{1} \ldots \mu_{n} \mu \nu}$ 's] determine its energy-momentum tensor together with the monopole $p_{T}^{\mu}$ and the spin dipole $S_{T}^{\mu \nu}$. The equations $\partial_{\mu} T^{\mu \nu} \stackrel{\circ}{=} 0$ are satisfied due to the equations of motion (C4) for $P_{T}^{\mu}$ and $S_{T}^{\mu \nu}$ [the so called Papapetrou-Dixon-Souriau equations given in Eqs. (5.18)] without the need of the equations of motion for the $J_{T}^{\mu_{1} \ldots \mu_{n} \mu \nu \rho \sigma}$. When all the multipoles $J_{T}^{\mu_{1} \ldots \mu_{n} \mu \nu \rho \sigma}$ are zero [or negligible] one speaks of a pole-dipole field configuration of the real Klein-Gordon field.

For the electromagnatic current of Section VIII the Hamilton equations (8.35) [equivalent to $\left.\partial_{\mu} \hat{J}_{\phi}^{\mu} \stackrel{\circ}{=} 0\right]$ give

$$
\begin{aligned}
& \frac{d \hat{q}_{\phi}}{d T_{s}} \stackrel{\circ}{=} 0 \\
& \frac{d \hat{q}_{\phi}^{\mu_{1} \ldots \mu_{n}}\left(T_{s}\right)}{d T_{s}} \stackrel{\circ}{=} \hat{j}_{\phi}^{\left(\mu_{1} \ldots \mu_{n}\right)}\left(T_{s}\right)-n u^{\left(\mu_{1}\right.}\left(p_{s}\right) \hat{q}_{\phi}^{\left.\mu_{2} \ldots \mu_{n}\right)}\left(T_{s}\right), \quad n \geq 1,
\end{aligned}
$$

By introducing the multipoles

$$
\hat{a}_{\phi}^{\mu_{1} \ldots \mu_{n} \mu}\left(T_{s}\right)=\hat{j}_{\phi}^{\mu_{1} \ldots \mu_{n} \mu}\left(T_{s}\right)-\hat{j}_{\phi}^{\left(\mu_{1} \ldots \mu_{n} \mu\right)}\left(T_{s}\right) .
$$

the previous equations become

$$
\begin{aligned}
\hat{j}_{\phi}^{\mu}\left(T_{s}\right) & \stackrel{\circ}{=} \hat{q}_{\phi} u^{\mu}\left(p_{s}\right)+\frac{d \hat{q}_{\phi}^{\mu}\left(T_{s}\right)}{d T_{s}}, \quad n=0, \\
\hat{j}_{\phi}^{\mu_{1} \ldots \mu_{n} \mu}\left(T_{s}\right) & \stackrel{\circ}{=} \hat{a}_{\phi}^{\mu_{1} \ldots \mu_{n} \mu}\left(T_{s}\right)+\hat{q}_{\phi}^{\left(\mu_{1} \ldots \mu_{n}\right.}\left(T_{s}\right) u^{\mu)}\left(p_{s}\right)+ \\
& +\frac{1}{n+1} \frac{d \hat{q}_{\phi}^{\mu_{1} \ldots \mu_{n} \mu}\left(T_{s}\right)}{d T_{s}}, \quad n \geq 1 .
\end{aligned}
$$


Let us introduce the new multipoles

$$
\begin{aligned}
\hat{m}_{\phi}^{\mu_{1} \ldots \mu_{n} \mu}\left(T_{s}\right)= & \hat{m}_{\phi}^{\left(\mu_{1} \ldots \mu_{n}\right) \mu}\left(T_{s}\right)= \\
= & \hat{a}_{\phi}^{\mu_{1} \ldots \mu_{n} \mu}\left(T_{s}\right)+\hat{q}_{\phi}^{\left(\mu_{1} \ldots \mu_{n}\right.}\left(T_{s}\right) u^{\mu)}\left(p_{s}\right)-u^{\left(\mu_{1}\right.}\left(p_{s}\right) \hat{q}_{\phi}^{\left.\mu_{2} \ldots \mu_{n}\right) \mu}\left(T_{s}\right)= \\
= & \hat{j}_{\phi}^{\mu_{1} \ldots \mu_{n} \mu}\left(T_{s}\right)-u^{\left(\mu_{1}\right.}\left(p_{s}\right) \hat{q}_{\phi}^{\left.\mu_{2} \ldots \mu_{n}\right) \mu}\left(T_{s}\right)-\frac{1}{n+1} \frac{d \hat{q}_{\phi}^{\mu_{1} \ldots \mu_{n} \mu}\left(T_{s}\right)}{d T_{s}}, \quad n \geq 1, \\
& \hat{m}_{\phi}^{\left(\mu_{1} \ldots \mu_{n} \mu\right)}\left(T_{s}\right)=0 \\
\hat{q}_{\phi}^{\mu_{1} \ldots \mu_{n}}\left(T_{s}\right)= & -n u_{\alpha}\left(p_{s}\right) \hat{m}_{\phi}^{\alpha \mu_{1} \ldots \mu_{n}}\left(T_{s}\right)=-n u_{\alpha}\left(p_{s}\right) \hat{m}_{\phi}^{\alpha\left(\mu_{1} \ldots \mu_{n}\right)}\left(T_{s}\right) .
\end{aligned}
$$

In Ref. 91 it is shown that we have

$$
\begin{aligned}
<\hat{J}_{\phi}^{\mu}, f> & =\int d T_{s} \int \frac{d^{4} k}{(2 \pi)^{4}} \tilde{f}(k) Q_{\phi}^{\mu}\left(k, T_{s}\right) \\
Q_{\phi}^{\mu}\left(k, T_{s}\right) & =e^{-i k \cdot x_{s}\left(T_{s}\right)}\left[\hat{q}_{\phi} u^{\mu}\left(p_{s}\right)+\sum_{n=1}^{\infty} \frac{(-i)^{n}}{n !} k_{\mu_{1}} \ldots k_{\mu_{n}} \hat{m}_{\phi}^{\mu_{1} \ldots \mu_{n} \mu}\left(T_{s}\right)\right]= \\
& =e^{-i k \cdot x_{s}\left(T_{s}\right)}\left[\hat{q}_{\phi} u^{\mu}\left(p_{s}\right)+\sum_{n=1}^{\infty} \frac{(-i)^{n} 2 n}{(n+1) !} k_{\mu_{1} \ldots} k_{\mu_{n}} \hat{Q}_{\phi}^{\left(\mu_{1} \ldots \mu_{n}\right) \mu}\left(T_{s}\right)\right]
\end{aligned}
$$

where the following new multipoles have been introduced [for $\mathrm{n}=0$ we get $Q_{\phi}^{[\mu \nu]}=\hat{j}_{\phi}^{[\mu \nu]}+$ $\left.\hat{q}_{\phi}^{[\mu} u^{\nu]}\left(p_{s}\right)\right]$

$$
\begin{aligned}
\hat{Q}_{\phi}^{\mu_{1} \ldots \mu_{n} \mu \nu}\left(T_{s}\right)= & \hat{Q}_{\phi}^{\left(\mu_{1} \ldots \mu_{n}\right)[\mu \nu]}\left(T_{s}\right)=\hat{m}_{\phi}^{\mu_{1} \ldots \mu_{n}[\mu \nu]}\left(T_{s}\right)= \\
= & \hat{j}_{\phi}^{\mu_{1} \ldots \mu_{n}[\mu \nu]}\left(T_{s}\right)+\frac{1}{n+1} \hat{q}_{\phi}^{\mu_{1} \ldots \mu_{n}[\mu}\left(T_{s}\right) u^{\nu]}\left(p_{s}\right), \quad n \geq 0 \\
& \hat{Q}_{\phi}^{\mu_{1} \ldots \mu_{n-1}\left[\mu_{n} \mu \nu\right]}\left(T_{s}\right)=0, \quad n \geq 1 \\
& u_{\mu_{1}}\left(p_{s}\right) \hat{Q}_{\phi}^{\mu_{1} \ldots \mu_{n} \mu \nu}\left(T_{s}\right)=0 \\
\hat{m}_{\phi}^{\mu_{1} \ldots \mu_{n} \mu}\left(T_{s}\right)= & \frac{2 n}{n+1} \hat{Q}_{\phi}^{\left(\mu_{1} \ldots \mu_{n}\right) \mu}\left(T_{s}\right) .
\end{aligned}
$$

The multipole $\hat{Q}_{\phi}^{\mu_{1} \ldots \mu_{n} \mu \nu}\left(T_{s}\right)$ is called the Dixon " $2^{n+1}$-pole electromagnetic moment tensor". The charge $q_{\phi}$ and these multipoles determine the electromagnetic current $\hat{J}_{\phi}^{\mu}$, whose conservation is simply equivalent to $\frac{d q_{\phi}}{d T_{s}} \stackrel{\circ}{=} 0$.

Therefore the complex Klein-Gordon field interacting with the electromagnetic field is an extended object defined by the inertial multipoles $\hat{P}_{\phi, A}^{\mu}, \hat{S}_{\phi, A}^{\mu \nu}, \hat{J}_{\phi, A}^{\mu_{1} \ldots \mu_{n} \mu \nu}$ and by the electromagnetic multipoles $q_{\phi}, \hat{Q}_{\phi}^{\mu_{1} \ldots \mu_{n} \mu}$.

Then in Ref. 9] there is a study of the Lorentz force $\hat{F}^{\mu}=-\hat{F}^{\mu \nu} \hat{J}_{\phi \nu}$ and it is shown that the equations $\partial_{\nu} \hat{T}_{\phi, A}^{\mu \nu} \stackrel{\circ}{=}-\hat{F}^{\mu}$ and $\partial_{\mu} \hat{J}_{\phi}^{\mu} \stackrel{\circ}{=} 0$ imply only the relations $\frac{d q_{\phi}}{d T_{s}} \stackrel{\circ}{=} 0$, $\frac{d \hat{P}_{\phi, A}^{\mu}}{d T_{s}} \stackrel{\circ}{=} \mathcal{F}^{\mu}\left(\hat{F}^{\alpha \beta}, \hat{Q}_{\phi}^{\nu_{1} \ldots \nu_{n} \rho \sigma}\right), \frac{d \hat{S}_{\phi, A}^{\mu \nu}}{d T_{s}} \stackrel{\circ}{=} \mathcal{F}^{\mu \nu}\left(\hat{F}^{\alpha \beta}, \hat{Q}_{\phi}^{\nu_{1} \ldots \nu_{n} \rho \sigma}\right)$ [see Dixon's paper for the criticism of the standard pole-dipole approximation]. 


\section{APPENDIX D: THE FESHBACH-VILLARS FORMALISM.}

As in Ref. [8], let us consider the two-component Feshbach-Villars formalism for the complex Klein-Gordon field [11] [see also Ref. [12,13]]. If we put ( $\tau_{i}$ are the Pauli matrices)

$$
\begin{aligned}
& \phi(\tau, \vec{\sigma})=\frac{1}{\sqrt{2}}[\varphi+\chi](\tau, \vec{\sigma})=\frac{1}{\sqrt{2}}\left(\phi_{1}+i \phi_{2}\right)(\tau, \vec{\sigma})= \\
& =\int d \tilde{q}\left[a(\tau, \vec{q}) e^{-i(\omega(q) \tau-\vec{q} \cdot \vec{\sigma})}+b^{*}(\tau, \vec{q}) e^{+i(\omega(q) \tau-\vec{q} \cdot \vec{\sigma})}\right], \\
& \frac{i}{m} \pi_{\phi^{*}}(\tau, \vec{\sigma})=\frac{1}{\sqrt{2}}[\varphi-\chi](\tau, \vec{\sigma})=\frac{i}{\sqrt{2} m}\left(\pi_{1}+i \pi_{2}\right)(\tau, \vec{\sigma})= \\
& =\int d \tilde{q} \frac{\omega(q)}{m}\left[a(\tau, \vec{q}) e^{-i(\omega(q) \tau-\vec{q} \cdot \vec{\sigma})}-b^{*}(\tau, \vec{q}) e^{+i(\omega(q) \tau-\vec{q} \cdot \vec{\sigma})}\right], \\
& \varphi(\tau, \vec{\sigma})=\frac{1}{\sqrt{2}}\left[\phi+\frac{i}{m} \pi_{\phi^{*}}\right](\tau, \vec{\sigma})=\frac{1}{2}\left[\phi_{1}+\frac{i}{m} \pi_{1}+i\left(\phi_{2}+\frac{i}{m} \pi_{2}\right)\right](\tau, \vec{\sigma})= \\
& =\frac{1}{\sqrt{2}} \int d \tilde{q}\left[\frac{m+\omega(q)}{m} a(\tau, \vec{q}) e^{-i(\omega(q) \tau-\vec{q} \cdot \vec{\sigma})}+\frac{m-\omega(q)}{m} b^{*}(\tau, \vec{q}) e^{+i(\omega(q) \tau-\vec{q} \cdot \vec{\sigma})}\right], \\
& \chi(\tau, \vec{\sigma})=\frac{1}{\sqrt{2}}\left[\phi-\frac{i}{m} \pi_{\phi^{*}}\right](\tau, \vec{\sigma})=\frac{1}{2}\left[\phi_{1}-\frac{i}{m} \pi_{1}+i\left(\phi_{2}-\frac{i}{m} \pi_{2}\right)\right](\tau, \vec{\sigma})= \\
& =\frac{1}{\sqrt{2}} \int d \tilde{q}\left[\frac{m-\omega(q)}{m} a(\tau, \vec{q}) e^{-i(\omega(q) \tau-\vec{q} \cdot \vec{\sigma})}+\frac{m+\omega(q)}{m} b^{*}(\tau, \vec{q}) e^{+i(\omega(q) \tau-\vec{q} \cdot \vec{\sigma})}\right], \\
& \Phi(\tau, \vec{\sigma})=\left(\begin{array}{c}
\varphi \\
\chi
\end{array}\right)(\tau, \vec{\sigma})=\int d \tilde{q} e^{-i \vec{q} \cdot \vec{\sigma}} \tilde{\Phi}(\tau, \vec{q})=\int d \tilde{q} e^{-i \vec{q} \cdot \vec{\sigma}}\left(\begin{array}{c}
\tilde{\varphi} \\
\tilde{\chi}
\end{array}\right)(\tau, \vec{q})= \\
& =\frac{1}{\sqrt{2}} \int d \tilde{q}\left[\left(\frac{\frac{m+\omega(q)}{m}}{\frac{m-\omega(q)}{m}}\right) a(\tau, \vec{q}) e^{-i(\omega(q) \tau-\vec{q} \cdot \vec{\sigma})}+\right. \\
& \left.+\left(\begin{array}{c}
\frac{m-\omega(q)}{m} \\
\frac{m+\omega(q)}{m}
\end{array}\right) b^{*}(\tau, \vec{q}) e^{+i(\omega(q) \tau-\vec{q} \cdot \vec{\sigma})}\right]
\end{aligned}
$$

the Hamilton equations for the Klein-Gordon field become

$$
\begin{aligned}
& i \partial_{\tau} \varphi(\tau, \vec{\sigma}) \stackrel{\circ}{=}\left[\frac{1}{2 m}(-i \vec{\partial})^{2}(\varphi+\chi)+m \varphi\right](\tau, \vec{\sigma}), \\
& i \partial_{\tau} \chi(\tau, \vec{\sigma}) \stackrel{\circ}{=}\left[-\frac{1}{2 m}(-i \vec{\partial})^{2}(\varphi+\chi)-m \chi\right](\tau, \vec{\sigma}),
\end{aligned}
$$

In the $2 \times 2$ matrix formalism we have

$$
\begin{aligned}
i \partial_{\tau} \Phi(\tau, \vec{\sigma}) & =\left[\frac{1}{2 m}(-i \vec{\partial})^{2}\left(\tau_{3}+i \tau_{2}\right)+m \tau_{3}\right] \Phi(\tau, \vec{\sigma})= \\
& =\hat{H} \Phi(\tau, \vec{\sigma}) .
\end{aligned}
$$

Since $\rho=\frac{i}{m} \Phi^{*} \tau_{3} \Phi=\frac{i}{m}\left(\varphi^{*} \varphi-\chi^{*} \chi\right)=\frac{i}{m}\left(\hat{\pi}_{\phi^{*}} \hat{\phi}^{*}-\hat{\pi}_{\phi} \hat{\phi}\right)$ is the density of the conserved charge $\frac{e}{m}$ (see the Gauss law), the normalization of $\Phi$ can be taken $\int d^{3} \sigma\left(\Phi^{*} \tau_{3} \Phi\right)(\tau, \vec{\sigma})=$ $\frac{q_{\phi}}{m}=\frac{N_{a \phi}-N_{b \phi}}{m}=\frac{e}{m}$. 
As shown in Ref. [1], the free Klein- Gordon field has the Hamiltonian $H_{o}=\frac{\vec{p}^{2}}{2 m}\left(\tau_{3}+\right.$ $\left.i \tau_{2}\right)+m \tau_{3}$ in the momentum representation and this Hamiltonian can be diagonalized $(\omega(p)=$ $\left.+\sqrt{m^{2}+\vec{p}^{2}}\right)$ with a $\tau_{3}$-unitary matrix $U(\omega(p))\left[U^{-1}=\tau_{3} U^{\dagger} \tau_{3} ; H_{o}\right.$ is $\tau_{3}$-hermitean, $H_{o}=$ $\left.\tau_{3} H^{\dagger} \tau_{3}\right]$

$$
\begin{aligned}
& H_{o, U}=U^{-1}(\omega(p)) H_{o} U(\omega(p))=\omega(p) \tau_{3}=\left(\begin{array}{cc}
\sqrt{m^{2}+\vec{p}^{2}} & 0 \\
0 & -\sqrt{m^{2}+\vec{p}^{2}}
\end{array}\right) \\
& \tilde{\Phi}_{U}(\tau, \vec{p})=U^{-1}(\omega(p)) \tilde{\Phi}(\tau, \vec{p})=\left(\begin{array}{c}
\tilde{\varphi}_{U} \\
\tilde{\chi}_{U}
\end{array}\right)(\tau, \vec{p})= \\
& =\frac{\sqrt{\omega(p)}}{m \sqrt{2 m}}\left[\left(\begin{array}{l}
1 \\
0
\end{array}\right) a(\tau, \vec{p}) e^{-i \omega(p) \tau}+\left(\begin{array}{l}
0 \\
1
\end{array}\right) b^{*}(\tau, \vec{p}) e^{+i \omega(p) \tau}\right], \\
& i \partial_{\tau} \tilde{\Phi}_{U}(\tau, \vec{p})=\left(\begin{array}{c}
i \partial_{\tau} \tilde{\varphi}_{U}(\tau, \vec{p}) \\
i \partial_{\tau} \tilde{\chi}_{U}(\tau, \vec{p})
\end{array}\right)=H_{o, U} \tilde{\Phi}_{U}(\tau, \vec{p})=\left(\begin{array}{c}
\omega(p) \tilde{\varphi}_{U}(\tau, \vec{p}) \\
-\omega(p) \tilde{\chi}_{U}(\tau, \vec{p})
\end{array}\right), \\
& U(\omega(p))=\frac{1}{2 \sqrt{m \omega(p)}}\left[(m+\omega(p)) 1+(m-\omega(p)) \tau_{1}\right], \\
& U^{-1}(\omega(p))=\frac{1}{2 \sqrt{m \omega(p)}}\left[(m+\omega(p)) 1-(m-\omega(p)) \tau_{1}\right] \\
& \left(\begin{array}{c}
\tilde{\varphi}_{U}(\tau, \vec{p}) \\
\tilde{\chi}_{U}(\tau, \vec{p})
\end{array}\right)=\frac{1}{2 \sqrt{m \omega(p)}}\left(\begin{array}{c}
(m+\omega(p)) \tilde{\varphi}(\tau, \vec{p})-(m-\omega(p)) \tilde{\chi}(\tau, \vec{p}) \\
(m+\omega(p)) \tilde{\chi}(\tau, \vec{p})-(m-\omega(p)) \tilde{\varphi}(\tau, \vec{p})
\end{array}\right)= \\
& =\frac{1}{\sqrt{2 m}}\left(\begin{array}{c}
\sqrt{\omega(p)} \tilde{\phi}(\tau, \vec{p})+\frac{i}{\sqrt{\omega(p)}} \tilde{\pi}_{\phi^{*}}(\tau, \vec{p}) \\
\sqrt{\omega(p)} \tilde{\phi}(\tau, \vec{p})-\frac{i}{\sqrt{\omega(p)}} \tilde{\pi}_{\phi^{*}}(\tau, \vec{p})
\end{array}\right)=\left(\begin{array}{c}
\tilde{\phi}_{a}(\tau, \vec{p}) \\
\tilde{\phi}_{b}^{*}(\tau, \vec{p})
\end{array}\right), \\
& \left(\begin{array}{c}
\tilde{\varphi}(\tau, \vec{p}) \\
\tilde{\chi}(\tau, \vec{p})
\end{array}\right)=U(\omega(p))\left(\begin{array}{c}
\tilde{\phi}_{a}(\tau, \vec{p}) \\
\tilde{\phi}_{b}^{*}(\tau, \vec{p})
\end{array}\right)= \\
& =\frac{1}{2 \sqrt{m \omega(p)}}\left(\begin{array}{c}
(m+\omega(p)) \tilde{\phi}_{a}(\tau, \vec{p})+(m-\omega(p)) \tilde{\phi}_{b}^{*}(\tau, \vec{p}) \\
(m+\omega(p)) \tilde{\phi}_{b}^{*}(\tau, \vec{p})+(m-\omega(p)) \tilde{\phi}_{a}(\tau, \vec{p})
\end{array}\right) .
\end{aligned}
$$

Therefore, we can introduce the following two Klein-Gordon fields

$$
\begin{aligned}
\phi_{a}(\tau, \vec{\sigma}) & =\varphi_{U}(\tau, \vec{\sigma})=\frac{1}{2 \sqrt{m \sqrt{m^{2}+\triangle}}}\left[\left(m+\sqrt{m^{2}+\triangle}\right) \varphi-\left(m-\sqrt{m^{2}+\triangle}\right) \chi\right](\tau, \vec{\sigma})= \\
& =\frac{1}{\sqrt{2 m \sqrt{m^{2}+\triangle}}}\left[\sqrt{m^{2}+\triangle} \phi+i \pi_{\phi^{*}}\right](\tau, \vec{\sigma})= \\
& =\sqrt{\frac{2}{m}} \int d \tilde{q} \sqrt{\omega(q)} a(\tau, \vec{q}) e^{-i(\omega(q) \tau-\vec{q} \cdot \vec{\sigma})}= \\
& =\sqrt{\frac{2}{m}} \int d \tilde{q} \sqrt{\omega(q)} \sqrt{F^{\tau}(q) \omega(q) P_{a \phi}^{\tau}-F(q) \vec{q} \cdot \vec{P}_{a \phi}+\mathcal{D}_{q} \mathbf{H}_{a}(\tau, \vec{q})} \\
& e^{i \int d \tilde{k} \int d \tilde{k}^{\prime} \mathbf{K}_{a}(\tau, \vec{k}) \mathcal{G}\left(\vec{k}, \vec{k}^{\prime}\right) \Delta\left(\vec{k}^{\prime}, \vec{q}\right)-i \omega(q)\left(\tau-X_{a \phi}^{\tau}\right)+i \vec{q} \cdot\left(\vec{\sigma}-\vec{X}_{a \phi}\right)=}= \\
& \sqrt{\frac{2}{m}} \int d \tilde{q} \sqrt{\omega(q)} \sqrt{F^{\tau}(q) \omega(q)\left[\frac{1}{2} P_{\phi}^{\tau}+Q_{\phi}^{\tau}\right]-F(q) \vec{q} \cdot\left[\frac{1}{2} \vec{P}_{\phi}+\vec{Q}_{\phi}\right]+\mathcal{D}_{q} \mathbf{H}_{a}(\tau, \vec{q})}
\end{aligned}
$$




$$
\begin{aligned}
& e^{+i \int d \tilde{k} \int d \tilde{k}^{\prime} \mathbf{K}_{a}(\tau, \vec{k}) \mathcal{G}\left(\vec{k}, \vec{k}^{\prime}\right) \Delta\left(\vec{k}^{\prime}, \vec{q}\right)-i \omega(q)\left(\tau-\left[X_{\phi}^{\tau}+\frac{1}{2} R_{\phi}^{\tau}\right]\right)+i \vec{q} \cdot\left(\vec{\sigma}-\left[\vec{X}_{\phi}+\frac{1}{2} \vec{R}_{\phi}\right]\right)} \\
& \phi_{b}(\tau, \vec{\sigma})=\chi_{U}^{*}(\tau, \vec{\sigma})=\frac{1}{2 \sqrt{m \sqrt{m^{2}+\triangle}}}\left[\left(m+\sqrt{m^{2}+\triangle}\right) \chi^{*}-\left(m-\sqrt{m^{2}+\triangle}\right) \varphi^{*}\right](\tau, \vec{\sigma})= \\
& =\frac{1}{\sqrt{2 m \sqrt{m^{2}+\triangle}}}\left[\sqrt{m^{2}+\triangle} \phi^{*}+i \pi\right](\tau, \vec{\sigma})= \\
& =\sqrt{\frac{2}{m}} \int d \tilde{q} \sqrt{\omega(q)} b(\tau, \vec{q}) e^{-i(\omega(q) \tau-\vec{q} \cdot \vec{\sigma})}= \\
& =\sqrt{\frac{2}{m}} \int d \tilde{q} \sqrt{\omega(q)} \sqrt{F^{\tau}(q) \omega(q) P_{b \phi}^{\tau}-F(q) \vec{q} \cdot \vec{P}_{b \phi}+\mathcal{D}_{q} \mathbf{H}_{b}(\tau, \vec{q})} \\
& e^{i \int d \tilde{k} \int d \tilde{k}^{\prime} \mathbf{K}_{b}(\tau, \vec{k}) \mathcal{G}\left(\vec{k}, \vec{k}^{\prime}\right) \Delta\left(\vec{k}^{\prime}, \vec{q}\right)-i \omega(q)\left(\tau-X_{b \phi}^{\tau}\right)+i \vec{q} \cdot\left(\vec{\sigma}-\vec{X}_{b \phi}\right)}= \\
& =\sqrt{\frac{2}{m}} \int d \tilde{q} \sqrt{\omega(q)} \sqrt{F^{\tau}(q) \omega(q)\left[\frac{1}{2} P_{\phi}^{\tau}-Q_{\phi}^{\tau}\right]-F(q) \vec{q} \cdot\left[\frac{1}{2} \vec{P}_{\phi}-\vec{Q}_{\phi}\right]+\mathcal{D}_{q} \mathbf{H}_{b}(\tau, \vec{q})} \\
& e^{+i \int d \tilde{k} \int d \tilde{k}^{\prime} \mathbf{K}_{b}(\tau, \vec{k}) \mathcal{G}\left(\vec{k}, \vec{k}^{\prime}\right) \Delta\left(\vec{k}^{\prime}, \vec{q}\right)-i \omega(q)\left(\tau-\left[X_{\phi}^{\tau}-\frac{1}{2} R_{\phi}^{\tau}\right]\right)+i \vec{q} \cdot\left(\vec{\sigma}-\left[\vec{X}_{\phi}-\frac{1}{2} \vec{R}_{\phi}\right]\right)}, \\
& \phi(\tau, \vec{\sigma})=\sqrt{\frac{m}{2}} \frac{1}{\left(m^{2}+\triangle\right)^{1 / 4}}\left[\phi_{a}(\tau, \vec{\sigma})+\phi_{b}^{*}(\tau, \vec{\sigma})\right]=\left[\phi^{*}(\tau, \vec{\sigma})\right]^{*}, \\
& \pi_{\phi^{*}}(\tau, \vec{\sigma})=\dot{\phi}(\tau, \vec{\sigma})=-i \sqrt{\frac{m}{2}}\left(m^{2}+\triangle\right)^{1 / 4}\left[\phi_{a}(\tau, \vec{\sigma})-\phi_{b}^{*}(\tau, \vec{\sigma})\right]=\left[\pi_{\phi}(\tau, \vec{\sigma})\right]^{*}, \\
& a(\tau, \vec{q})=\sqrt{2 m \omega(q)} \int d^{3} \sigma \phi_{a}(\tau, \vec{\sigma}) e^{i(\omega(q) \tau-\vec{q} \cdot \vec{\sigma})}= \\
& =\int d^{3} \sigma\left[\omega(q) \phi(\tau, \vec{\sigma})+i \pi_{\phi^{*}}(\tau, \vec{\sigma})\right] e^{i(\omega(q) \tau-\vec{q} \cdot \vec{\sigma})}, \\
& a^{*}(\tau, \vec{q})=\sqrt{2 m \omega(q)} \int d^{3} \sigma \phi_{a}^{*}(\tau, \vec{\sigma}) e^{-i(\omega(q) \tau-\vec{q} \cdot \vec{\sigma})}= \\
& =\int d^{3} \sigma\left[\omega(q) \phi^{*}(\tau, \vec{\sigma})+i \pi_{\phi}(\tau, \vec{\sigma})\right] e^{-i(\omega(q) \tau-\vec{q} \cdot \vec{\sigma})}, \\
& b(\tau, \vec{q})=\sqrt{2 m \omega(q)} \int d^{3} \sigma \phi_{b}(\tau, \vec{\sigma}) e^{i(\omega(q) \tau-\vec{q} \cdot \vec{\sigma})}= \\
& =\int d^{3} \sigma\left[\omega(q) \phi(\tau, \vec{\sigma})-i \pi_{\phi^{*}}(\tau, \vec{\sigma})\right] e^{i(\omega(q) \tau-\vec{q} \cdot \vec{\sigma})}, \\
& b^{*}(\tau, \vec{q})=\sqrt{2 m \omega(q)} \int d^{3} \sigma \phi_{b}^{*}(\tau, \vec{\sigma}) e^{-i(\omega(q) \tau-\vec{q} \cdot \vec{\sigma})}= \\
& =\int d^{3} \sigma\left[\omega(q) \phi^{*}(\tau, \vec{\sigma})+i \pi_{\phi}(\tau, \vec{\sigma})\right] e^{-i(\omega(q) \tau-\vec{q} \cdot \vec{\sigma})} .
\end{aligned}
$$

They are solutions of the square-root Klein-Gordon equation [see Ref. 25] for the study of this equation by using the theory of pseudodifferential operators] with both the energy and the electric charge of the same sign: positive for $\phi_{a}$ and negative for $\phi_{b}$.

$$
\begin{aligned}
& i \partial_{\tau} \phi_{a}(\tau, \vec{\sigma}) \stackrel{\circ}{=} \sqrt{m^{2}+\triangle \phi_{a}}(\tau, \vec{\sigma}), \quad q_{\phi}=N_{a \phi}, \\
& i \partial_{\tau} \phi_{b}(\tau, \vec{\sigma}) \stackrel{\circ}{=}-\sqrt{m^{2}+\triangle} \phi_{b}(\tau, \vec{\sigma}), \quad q_{\phi}=-N_{b \phi} .
\end{aligned}
$$

Like in the case of the Foldy-Wouthuysen transformation for particles of spin $1 / 2$, also in the spin 0 case the exact diagonalization of the Hamiltonian cannot be achieved in presence 
of an arbitrary external electromagnetic field [11], when Eqs.(D2) become [see Ref. [8]]

$$
\begin{aligned}
& i \partial_{\tau} \varphi(\tau, \vec{\sigma}) \stackrel{\circ}{=}\left[\frac{1}{2 m}\left(-i \vec{\partial}-e \vec{A}_{\perp}\right)^{2}(\varphi+\chi)+(m+K) \varphi\right](\tau, \vec{\sigma}), \\
& i \partial_{\tau} \chi(\tau, \vec{\sigma}) \stackrel{\circ}{=}\left[-\frac{1}{2 m}\left(-i \vec{\partial}-e \vec{A}_{\perp}\right)^{2}(\varphi+\chi)+(-m+K) \chi\right](\tau, \vec{\sigma}), \\
& K(\tau, \vec{\sigma})=-\frac{e^{2}}{4 \pi} \int d^{3} \sigma_{1} \frac{i\left(\hat{\pi}_{\phi^{*}} \hat{\phi}^{*}-\hat{\pi}_{\phi} \hat{\phi}\right)\left(\tau, \vec{\sigma}_{1}\right)}{\left|\vec{\sigma}-\vec{\sigma}_{1}\right|}= \\
& =-\frac{m e^{2}}{4 \pi} \int d^{3} \sigma_{1} \frac{\left(\varphi^{*} \varphi-\chi^{*} \chi\right)\left(\tau, \vec{\sigma}_{1}\right)}{\left|\vec{\sigma}-\vec{\sigma}_{1}\right|}= \\
& =-\frac{m e^{2}}{4 \pi} \int d^{3} \sigma_{1} \frac{\left(\Phi^{*} \tau_{3} \Phi\right)\left(\tau, \vec{\sigma}_{1}\right)}{\left|\vec{\sigma}-\vec{\sigma}_{1}\right|}=\int d \tilde{k} K(\tau, \vec{k}) e^{-i \vec{k} \cdot \vec{\sigma}} .
\end{aligned}
$$

In this case, Eq.(D3) assumes the following form after Fourier transform

$$
\begin{aligned}
i \partial_{\tau} \tilde{\Phi}(\tau, \vec{p}) & =\tilde{H} \tilde{\Phi}(\tau, \vec{p}), \\
\tilde{H} & =\frac{1}{2 m}\left[\vec{p}-e \int d^{3} k \vec{A}_{\perp}(\tau, \vec{k}) e^{-\vec{k} \cdot \vec{\partial}}\right]^{2}\left(\tau_{3}+i \tau_{2}\right)+m \tau_{3}+ \\
& +\int d^{3} k K(\tau, \vec{k}) e^{-\vec{k} \cdot \vec{\partial}} \mathbb{1} .
\end{aligned}
$$

If we put $\tilde{\Phi}(\tau, \vec{p})=U(\omega(p)) \tilde{\Phi}_{U}(\tau, \vec{p})$ with the same $U(\vec{p})$ of the free case, we get [see Ref. [11]]

$$
\begin{aligned}
i \partial_{\tau} \tilde{\Phi}_{U}(\tau, \vec{p})= & \sqrt{m^{2}+\vec{p}^{2}} \tau_{3} \tilde{\Phi}_{U}(\tau, \vec{p})+ \\
+ & \int d^{3} k K(\tau, \vec{p}-\vec{k}) \frac{\left(\sqrt{m^{2}+\vec{p}^{2}}+\sqrt{m^{2}+\vec{k}^{2}}\right) \mathbb{1}+\left(\sqrt{m^{2}+\vec{p}^{2}}-\sqrt{m^{2}+\vec{k}^{2}}\right) \tau_{1}}{2 \sqrt{\sqrt{m^{2}+\vec{p}^{2}} \sqrt{m^{2}+\vec{k}^{2}}}} \\
& \mathbb{1} \tilde{\Phi}_{U}(\tau, \vec{k})+ \\
+ & \left.\int d^{3} k \frac{e}{m} \vec{k} \cdot \overrightarrow{A_{\perp}}(\tau, \vec{p}-\vec{k})+\frac{e^{2}}{2 m}\left(\vec{A}_{\perp}^{2}\right)(\tau, \vec{p}-\vec{k})\right] \\
& \left(\mathbb{1}+\tau_{1}\right) \tilde{\Phi}_{U}(\tau, \vec{k}),
\end{aligned}
$$

where $\left(\vec{A}_{\perp}^{2}\right)(\tau, \vec{p})$ means the Fourier transform of $\vec{A}_{\perp}^{2}(\tau, \vec{\sigma})$.

In ref. [1], it is shown that this Hamiltonian cannot be diagonalized, because the separation of positive and negative energies is inhibited by effects which (in a second quantized formalism) can be ascribed to the vacuum polarization, namely to the pair production. This (i.e. the nonseparability of positive and negative energies) is also the source of the zitterbewegung effects for localized Klein-Gordon wave packets as discussed in Ref. [11].

With an integration by parts, the constraint $\mathcal{H}(\tau)$ of Eq. 8.32) can be rewritten as

$$
\begin{aligned}
\epsilon_{s} & -\frac{1}{2} \int d^{3} \sigma\left(\vec{\pi}_{\perp}^{2}+\vec{B}^{2}\right)(\tau, \vec{\sigma})- \\
& -\int d^{3} \sigma \Phi^{*}(\tau, \vec{\sigma}) \tau_{3}\left[\frac{1}{2}\left(-i \vec{\partial}-e \vec{A}_{\perp}(\tau, \vec{\sigma})\right)^{2}\left(\tau_{3}+i \tau_{2}\right)+m^{2} \tau_{3}\right] \Phi(\tau, \vec{\sigma})+
\end{aligned}
$$




$$
+\int d^{3} \sigma \Phi^{*}(\tau, \vec{\sigma}) \tau_{3}\left[\frac{e^{2} m^{2}}{8 \pi} \int d^{3} \sigma_{1} \frac{\left(\Phi^{*} \tau_{3} \Phi\right)\left(\tau, \vec{\sigma}_{1}\right)}{\left|\vec{\sigma}-\vec{\sigma}_{1}\right|} \mathbb{1}\right] \Phi(\tau, \vec{\sigma}) \approx 0
$$

If we suppose that $\Phi(\tau, \vec{\sigma})$ is normalized to $\int d^{3} \sigma \Phi^{*}(\tau, \vec{\sigma}) \tau_{3} \Phi(\tau, \vec{\sigma})=1 / m$ [this is a charge normalization compatible with the nonlinear equations of motion, because the electric charge is conserved], we can rewrite the previous formula as

$$
\begin{aligned}
& \int d^{3} \sigma \Phi^{*}(\tau, \vec{\sigma}) \tau_{3}\left\{\left[\epsilon_{s}-\frac{1}{2} \int d^{3} \sigma_{1}\left(\vec{\pi}_{\perp}^{2}+\vec{B}^{2}\right)\left(\tau, \vec{\sigma}_{1}\right)+\right.\right. \\
+ & \left.\frac{e^{2} m}{8 \pi} \int d^{3} \sigma_{1} \frac{\left(\Phi^{*} \tau_{3} \Phi\right)\left(\tau, \vec{\sigma}_{1}\right)}{\left|\vec{\sigma}-\vec{\sigma}_{1}\right|}\right] \mathbb{1}- \\
- & {\left.\left[\frac{1}{2 m}\left(-i \vec{\partial}-e \vec{A}_{\perp}(\tau, \vec{\sigma})\right)^{2}\left(\tau_{3}+i \tau_{2}\right)+m \tau_{3}\right]\right\} \Phi(\tau, \vec{\sigma}) \approx 0 . }
\end{aligned}
$$

If we assume that the nonlinear equations for the reduced Klein-Gordon field have solutions of the form $\Phi(\tau, \vec{\sigma})=\Phi(\tau, \vec{p}) e^{i \vec{p} \cdot \vec{\sigma}}+\Phi_{1}(\tau, \vec{\sigma})$ with $\Phi_{1}$ negligible, namely that the global form of the nonlinear wave admits a sensible eikonal approximation, then, neglecting $\Phi_{1}$, we get approximately

$$
\begin{aligned}
& \int d^{3} \sigma \Phi^{*}(\tau, \vec{\sigma}) \tau_{3}\left\{\left[\epsilon_{s}-\frac{1}{2} \int d^{3} \sigma\left(\vec{\pi}_{\perp}^{2}+\vec{B}^{2}\right)(\tau, \vec{\sigma})+\right.\right. \\
+ & \left.\frac{e^{2} m}{8 \pi} \int d^{3} \sigma_{1} \frac{\left(\Phi^{*} \tau_{3} \Phi\right)\left(\tau, \vec{\sigma}_{1}\right)}{\left|\vec{\sigma}-\vec{\sigma}_{1}\right|}\right] \mathbb{1}- \\
- & {\left.\left[\frac{1}{2 m}\left(\vec{p}-e \vec{A}_{\perp}(\tau, \vec{\sigma})\right)^{2}\left(\tau_{3}+i \tau_{2}\right)+m \tau_{3}\right]\right\} \Phi(\tau, \vec{\sigma}) \approx 0 . }
\end{aligned}
$$

If we now redefine $\Phi_{U}(\tau, \vec{\sigma})=U^{-1}\left(\sqrt{m^{2}+\left(\vec{p}-e \overrightarrow{A_{\perp}}(\tau, \vec{\sigma})\right)^{2}}\right) \Phi(\tau, \vec{\sigma})$ with the same $\mathrm{U}$ of Eq.(D4), we get

$$
\begin{aligned}
& \int d^{3} \sigma \Phi_{U}^{*}(\tau, \vec{\sigma}) \tau_{3}\left(\begin{array}{cc}
\mathcal{H}_{+}(\tau, \vec{\sigma}) & 0 \\
0 & \mathcal{H}_{-}(\tau, \vec{\sigma})
\end{array}\right) \Phi_{U}(\tau, \vec{\sigma})= \\
= & \int d^{3} \sigma\left[\phi_{a}^{*} \mathcal{H}_{+} \phi_{a}-\phi_{b}^{*} \mathcal{H}_{-} \phi_{b}\right](\tau, \vec{\sigma}) \approx 0 \\
& \mathcal{H}_{ \pm}(\tau, \vec{\sigma})=\epsilon_{s} \mp \sqrt{m^{2}+\left(\vec{p} \mp|e| \vec{A}_{\perp}(\tau, \vec{\sigma})\right)^{2}}+ \\
+ & \frac{e^{2} m}{8 \pi} \int d^{3} \sigma_{1} \frac{\left(\Phi^{*} \tau_{3} \Phi\right)\left(\tau, \vec{\sigma}_{1}\right)}{\left|\vec{\sigma}-\vec{\sigma}_{1}\right|}-\frac{1}{2} \int d^{3} \sigma_{1}\left(\vec{\pi}_{\perp}^{2}+\vec{B}^{2}\right)\left(\tau, \vec{\sigma}_{1}\right) .
\end{aligned}
$$

where $\mathcal{H}_{ \pm}(\tau, \vec{\sigma}) \approx 0$ are the form for $\mathrm{N}=1$ of the constraints

$$
\begin{aligned}
\mathcal{H}(\tau) & =\epsilon_{s}-\left\{\sum_{i=1}^{N} \eta_{i} \sqrt{m_{i}^{2}+\left(\check{\vec{\kappa}}_{i}(\tau)-Q_{i} \vec{A}_{\perp}\left(\tau, \vec{\eta}_{i}(\tau)\right)\right)^{2}}+\right. \\
& \left.\left.+\sum_{i \neq j} \frac{Q_{i} Q_{j}}{4 \pi\left|\vec{\eta}_{i}(\tau)-\vec{\eta}_{j}(\tau)\right|}+\int d^{3} \sigma \frac{1}{2}^{2} \check{\breve{\pi}}_{\perp}^{2}(\tau, \vec{\sigma})+\check{\vec{B}}^{2}(\tau, \vec{\sigma})\right]\right\} \approx 0, \\
\overrightarrow{\mathcal{H}}_{p}(\tau) & =\check{\vec{\kappa}}_{+}(\tau)+\int d^{3} \sigma\left[\check{\vec{\pi}}_{\perp} \times \check{\vec{B}}\right](\tau, \vec{\sigma}) \approx 0
\end{aligned}
$$


given in Ref. [8] for the invariant mass of charged scalar particles plus the electromagnetic field, which was determined in Ref. [臬] for the two possible signs of the energy $\eta= \pm$ [with the Grassmann charges $Q_{i}$ replaced with $\left.\pm|e|\right]$, when evaluated at $\vec{\sigma}=\vec{\eta}_{i}(\tau)$. Under the square root there is only a magnetic coupling to $\vec{A}_{\perp}$ : in this way the quoted problems of the interconnession of electric fields with pair production are avoided. The Klein-Gordon selfenergy should go in the particle limit (eikonal approximation of field theory) in the Coulomb self-energy of the classical particle, which is absent in Ref. [1] because it is regularized by assuming that the particle electric charge $Q$ is pseudoclassically described by Grassmann variables so that $Q^{2}=0$. Therefore, the particle description of Ref. [1] is valid only when one disregards the effects induced by vacuum polarization and pair production and uses a strong eikonal approximation neglecting diffractive effects. 


\section{APPENDIX E: THE INVARIANT MASS IN TERMS OF $\hat{A}, \hat{B}$.}

The invariant mass $M$ of Eq. 8.40) has the following form in terms of the Fourier coefficients $\hat{a}$ and $\hat{b}$ [see Eqs.(D5) for their expression in terms of the final canonical variables]:

$$
\begin{aligned}
& M=\frac{1}{2} \int d^{3} \sigma\left(\vec{\pi}_{\perp}^{2}+\vec{B}^{2}\right)(\tau, \vec{\sigma})+\hat{P}_{\phi}^{\tau}(\tau)+ \\
& +m \int d \tilde{q}_{1} d \tilde{q}_{2} \int d^{3} \sigma\left[e^{i\left(\omega\left(q_{1}\right) \tau-\vec{q}_{1} \cdot \vec{\sigma}\right)} \hat{a}^{*}\left(\tau, \vec{q}_{1}\right)+e^{i\left(\omega\left(q_{1}\right) \tau-\vec{q}_{1} \cdot \vec{\sigma}\right)} \hat{b}\left(\tau, \vec{q}_{1}\right)\right] \\
& \left(-2 e \vec{q}_{2} \cdot \vec{A}_{\perp}(\tau, \vec{\sigma})\left[e^{-i\left(\omega\left(q_{2}\right) \tau-\vec{q}_{2} \cdot \vec{\sigma}\right)} \hat{a}\left(\tau, \vec{q}_{2}\right)-e^{i\left(\omega\left(q_{2}\right) \tau-\vec{q}_{2} \cdot \vec{\sigma}\right)} \hat{b}^{*}\left(\tau, \vec{q}_{2}\right)\right]+\right. \\
& \left.+e^{2} \vec{A}_{\perp}^{2}(\tau, \vec{\sigma})\left[e^{-i\left(\omega\left(q_{2}\right) \tau-\overrightarrow{q_{2}} \cdot \vec{\sigma}\right)} \hat{a}\left(\tau, \vec{q}_{2}\right)+e^{i\left(\omega\left(q_{2}\right) \tau-\vec{q}_{2} \cdot \vec{\sigma}\right)} \hat{b}^{*}\left(\tau, \vec{q}_{2}\right)\right]\right)- \\
& -\frac{e^{2} m^{2}}{8}(2 \pi)^{3} \int d \tilde{q}_{1} d \tilde{q}_{2} d \tilde{q}_{3} d \tilde{q}_{4} \sqrt{\frac{\omega\left(q_{1}\right) \omega\left(q_{3}\right)}{\omega\left(q_{2}\right) \omega\left(q_{4}\right)}} \\
& \left(\frac{1}{\left(\vec{q}_{1}-\vec{q}_{2}\right)^{2}}\right. \\
& {\left[\delta ^ { 3 } ( \vec { q _ { 1 } } - \vec { q _ { 2 } } + \vec { q _ { 3 } } - \vec { q _ { 4 } } ) \left(e^{-i\left[\omega\left(q_{1}\right)-\omega\left(q_{2}\right)+\omega\left(q_{3}\right)-\omega\left(q_{4}\right)\right] \tau}\right.\right.} \\
& \left(\hat{a}\left(\tau, \vec{q}_{1}\right) \hat{a}^{*}\left(\tau, \vec{q}_{2}\right)+\hat{b}\left(\tau, \vec{q}_{1}\right) \hat{b}^{*}\left(\tau, \vec{q}_{2}\right)\right)\left(\hat{a}\left(\tau, \vec{q}_{3}\right) \hat{a}^{*}\left(\tau, \vec{q}_{4}\right)+\hat{b}\left(\tau, \vec{q}_{3}\right) \hat{b}^{*}\left(\tau, \vec{q}_{4}\right)\right)+ \\
& +e^{i\left[\omega\left(q_{1}\right)-\omega\left(q_{2}\right)+\omega\left(q_{3}\right)-\omega\left(q_{4}\right)\right] \tau} \\
& \left.\left(\hat{a}^{*}\left(\tau, \vec{q}_{1}\right) \hat{a}\left(\tau, \vec{q}_{2}\right)+\hat{b}^{*}\left(\tau, \overrightarrow{q_{1}}\right) \hat{b}\left(\tau, \vec{q}_{2}\right)\right)\left(\hat{a}^{*}\left(\tau, \vec{q}_{3}\right) \hat{a}\left(\tau, \vec{q}_{4}\right)+\hat{b}^{*}\left(\tau, \vec{q}_{3}\right) \hat{b}\left(\tau, \vec{q}_{4}\right)\right)\right)- \\
& -\delta^{3}\left(\vec{q}_{1}-\vec{q}_{2}-\vec{q}_{3}+\vec{q}_{4}\right)\left(e^{-i\left[\omega\left(q_{1}\right)-\omega\left(q_{2}\right)-\omega\left(q_{3}\right)+\omega\left(q_{4}\right)\right] \tau}\right. \\
& \left(\hat{a}\left(\tau, \vec{q}_{1}\right) \hat{a}^{*}\left(\tau, \vec{q}_{2}\right)+\hat{b}\left(\tau, \vec{q}_{1}\right) \hat{b}^{*}\left(\tau, \vec{q}_{2}\right)\right)\left(\hat{a}^{*}\left(\tau, \vec{q}_{3}\right) \hat{a}\left(\tau, \vec{q}_{4}\right)+\hat{b}^{*}\left(\tau, \vec{q}_{3}\right) \hat{b}\left(\tau, \vec{q}_{4}\right)\right)+ \\
& +e^{i\left[\omega\left(q_{1}\right)-\omega\left(q_{2}\right)-\omega\left(q_{3}\right)+\omega\left(q_{4}\right)\right] \tau} \\
& \left.\left(\hat{a}^{*}\left(\tau, \vec{q}_{1}\right) \hat{a}\left(\tau, \vec{q}_{2}\right)+\hat{b}^{*}\left(\tau, \vec{q}_{1}\right) \hat{b}\left(\tau, \vec{q}_{2}\right)\right)\left(\hat{a}\left(\tau, \vec{q}_{3}\right) \hat{a}^{*}\left(\tau, \vec{q}_{4}\right)+\hat{b}\left(\tau, \vec{q}_{3}\right) \hat{b}^{*}\left(\tau, \vec{q}_{4}\right)\right)\right)+ \\
& +\delta^{3}\left(\vec{q}_{1}-\vec{q}_{2}+\vec{q}_{3}+\vec{q}_{4}\right)\left(e^{-i\left[\omega\left(q_{1}\right)-\omega\left(q_{2}\right)+\omega\left(q_{3}\right)+\omega\left(q_{4}\right)\right] \tau}\right. \\
& \left(\hat{a}\left(\tau, \vec{q}_{1}\right) \hat{a}^{*}\left(\tau, \vec{q}_{2}\right)+\hat{b}\left(\tau, \vec{q}_{1}\right) \hat{b}^{*}\left(\tau, \vec{q}_{2}\right)\right)\left(\hat{a}\left(\tau, \vec{q}_{3}\right) \hat{b}\left(\tau, \vec{q}_{4}\right)+\hat{b}\left(\tau, \vec{q}_{3}\right) \hat{a}\left(\tau, \vec{q}_{4}\right)\right)+ \\
& +e^{i\left[\omega\left(q_{1}\right)-\omega\left(q_{2}\right)+\omega\left(q_{3}\right)+\omega\left(q_{4}\right)\right] \tau} \\
& \left.\left(\hat{a}^{*}\left(\tau, \vec{q}_{1}\right) \hat{a}\left(\tau, \vec{q}_{2}\right)+\hat{b}^{*}\left(\tau, \vec{q}_{1}\right) \hat{b}\left(\tau, \vec{q}_{2}\right)\right)\left(\hat{a}^{*}\left(\tau, \vec{q}_{3}\right) \hat{b}^{*}\left(\tau, \vec{q}_{4}\right)+\hat{b}^{*}\left(\tau, \vec{q}_{3}\right) \hat{a}^{*}\left(\tau, \vec{q}_{4}\right)\right)\right)- \\
& -\delta^{3}\left(\vec{q}_{1}-\vec{q}_{2}-\vec{q}_{3}-\vec{q}_{4}\right)\left(e^{-i\left[\omega\left(q_{1}\right)-\omega\left(q_{2}\right)-\omega\left(q_{3}\right)-\omega\left(q_{4}\right)\right] \tau}\right. \\
& \left(\hat{a}\left(\tau, \vec{q}_{1}\right) \hat{a}^{*}\left(\tau, \vec{q}_{2}\right)+\hat{b}\left(\tau, \vec{q}_{1}\right) \hat{b}^{*}\left(\tau, \vec{q}_{2}\right)\right)\left(\hat{a}^{*}\left(\tau, \vec{q}_{3}\right) \hat{b}^{*}\left(\tau, \vec{q}_{4}\right)+\hat{b}^{*}\left(\tau, \vec{q}_{3}\right) \hat{a}^{*}\left(\tau, \vec{q}_{4}\right)\right)+ \\
& +e^{i\left[\omega\left(q_{1}\right)-\omega\left(q_{2}\right)-\omega\left(q_{3}\right)-\omega\left(q_{4}\right)\right] \tau} \\
& \left.\left.\left(\hat{a}^{*}\left(\tau, \vec{q}_{1}\right) \hat{a}\left(\tau, \vec{q}_{2}\right)+\hat{b}^{*}\left(\tau, \vec{q}_{1}\right) \hat{b}\left(\tau, \vec{q}_{2}\right)\right)\left(\hat{a}\left(\tau, \vec{q}_{3}\right) \hat{b}\left(\tau, \vec{q}_{4}\right)+\hat{b}\left(\tau, \vec{q}_{3}\right) \hat{a}\left(\tau, \vec{q}_{4}\right)\right)\right)\right]+ \\
& +\frac{1}{\left(\vec{q}_{1}+\vec{q}_{2}\right)^{2}} \\
& {\left[\delta ^ { 3 } ( \vec { q _ { 1 } } + \vec { q _ { 2 } } + \vec { q _ { 3 } } - \vec { q _ { 4 } } ) \left(e^{-i\left[\omega\left(q_{1}\right)+\omega\left(q_{2}\right)+\omega\left(q_{3}\right)-\omega\left(q_{4}\right)\right] \tau}\right.\right.} \\
& \left(\hat{a}\left(\tau, \vec{q}_{1}\right) \hat{b}\left(\tau, \vec{q}_{2}\right)+\hat{b}\left(\tau, \vec{q}_{1}\right) \hat{a}\left(\tau, \vec{q}_{2}\right)\right)\left(\hat{a}\left(\tau, \vec{q}_{3}\right) \hat{a}^{*}\left(\tau, \vec{q}_{4}\right)+\hat{b}\left(\tau, \vec{q}_{3}\right) \hat{b}^{*}\left(\tau, \vec{q}_{4}\right)\right)+ \\
& +e^{i\left[\omega\left(q_{1}\right)+\omega\left(q_{2}\right)+\omega\left(q_{3}\right)-\omega\left(q_{4}\right)\right] \tau} \\
& \left.\left(\hat{a}^{*}\left(\tau, \vec{q}_{1}\right) \hat{b}^{*}\left(\tau, \vec{q}_{2}\right)+\hat{b}^{*}\left(\tau, \vec{q}_{1}\right) \hat{a}^{*}\left(\tau, \vec{q}_{2}\right)\right)\left(\hat{a}^{*}\left(\tau, \vec{q}_{3}\right) \hat{a}\left(\tau, \vec{q}_{4}\right)+\hat{b}^{*}\left(\tau, \overrightarrow{q_{3}}\right) \hat{b}\left(\tau, \vec{q}_{4}\right)\right)\right)-
\end{aligned}
$$




$$
\begin{aligned}
& -\delta^{3}\left(\vec{q}_{1}+\vec{q}_{2}-\vec{q}_{3}+\vec{q}_{4}\right)\left(e^{-i\left[\omega\left(q_{1}\right)+\omega\left(q_{2}\right)-\omega\left(q_{3}\right)+\omega\left(q_{4}\right)\right] \tau}\right. \\
& \left(\hat{a}\left(\tau, \vec{q}_{1}\right) \hat{b}\left(\tau, \vec{q}_{2}\right)+\hat{b}\left(\tau, \vec{q}_{1}\right) \hat{a}\left(\tau, \vec{q}_{2}\right)\right)\left(\hat{a}^{*}\left(\tau, \vec{q}_{3}\right) \hat{a}\left(\tau, \vec{q}_{4}\right)+\hat{b}^{*}\left(\tau, \vec{q}_{3}\right) \hat{b}\left(\tau, \vec{q}_{4}\right)\right)+ \\
& +e^{i\left[\omega\left(q_{1}\right)+\omega\left(q_{2}\right)-\omega\left(q_{3}\right)+\omega\left(q_{4}\right)\right] \tau} \\
& \left.\left(\hat{a}^{*}\left(\tau, \vec{q}_{1}\right) \hat{b}^{*}\left(\tau, \vec{q}_{2}\right)+\hat{b}^{*}\left(\tau, \vec{q}_{1}\right) \hat{a}^{*}\left(\tau, \vec{q}_{2}\right)\right)\left(\hat{a}\left(\tau, \vec{q}_{3}\right) \hat{a}^{*}\left(\tau, \vec{q}_{4}\right)+\hat{b}\left(\tau, \vec{q}_{3}\right) \hat{b}^{*}\left(\tau, \vec{q}_{4}\right)\right)\right)+ \\
& +\delta^{3}\left(\vec{q}_{1}+\vec{q}_{2}+\vec{q}_{3}+\vec{q}_{4}\right)\left(e^{-i\left[\omega\left(q_{1}\right)+\omega\left(q_{2}\right)+\omega\left(q_{3}\right)+\omega\left(q_{4}\right)\right] \tau}\right. \\
& \left(\hat{a}\left(\tau, \vec{q}_{1}\right) \hat{b}\left(\tau, \vec{q}_{2}\right)+\hat{b}\left(\tau, \vec{q}_{1}\right) \hat{a}\left(\tau, \vec{q}_{2}\right)\right)\left(\hat{a}\left(\tau, \vec{q}_{3}\right) \hat{b}\left(\tau, \vec{q}_{4}\right)+\hat{b}\left(\tau, \vec{q}_{3}\right) \hat{a}\left(\tau, \vec{q}_{4}\right)\right)+ \\
& +e^{i\left[\omega\left(q_{1}\right)+\omega\left(q_{2}\right)+\omega\left(q_{3}\right)+\omega\left(q_{4}\right)\right] \tau} \\
& \left.\left(\hat{a}^{*}\left(\tau, \vec{q}_{1}\right) \hat{b}^{*}\left(\tau, \vec{q}_{2}\right)+\hat{b}^{*}\left(\tau, \vec{q}_{1}\right) \hat{a}^{*}\left(\tau, \vec{q}_{2}\right)\right)\left(\hat{a}^{*}\left(\tau, \vec{q}_{3}\right) \hat{b}^{*}\left(\tau, \vec{q}_{4}\right)+\hat{b}^{*}\left(\tau, \vec{q}_{3}\right) \hat{a}^{*}\left(\tau, \vec{q}_{4}\right)\right)\right)- \\
& -\delta^{3}\left(\vec{q}_{1}+\vec{q}_{2}-\vec{q}_{3}-\vec{q}_{4}\right)\left(e^{-i\left[\omega\left(q_{1}\right)+\omega\left(q_{2}\right)-\omega\left(q_{3}\right)-\omega\left(q_{4}\right)\right] \tau}\right. \\
& \left(\hat{a}\left(\tau, \vec{q}_{1}\right) \hat{b}\left(\tau, \vec{q}_{2}\right)+\hat{b}\left(\tau, \vec{q}_{1}\right) \hat{a}\left(\tau, \vec{q}_{2}\right)\right)\left(\hat{a}^{*}\left(\tau, \vec{q}_{3}\right) \hat{b}^{*}\left(\tau, \vec{q}_{4}\right)+\hat{b}^{*}\left(\tau, \vec{q}_{3}\right) \hat{a}^{*}\left(\tau, \vec{q}_{4}\right)\right)+ \\
& +e^{i\left[\omega\left(q_{1}\right)+\omega\left(q_{2}\right)-\omega\left(q_{3}\right)-\omega\left(q_{4}\right)\right] \tau} \\
& \left.\left.\left.\left(\hat{a}^{*}\left(\tau, \vec{q}_{1}\right) \hat{b}^{*}\left(\tau, \vec{q}_{2}\right)+\hat{b}^{*}\left(\tau, \vec{q}_{1}\right) \hat{a}^{*}\left(\tau, \vec{q}_{2}\right)\right)\left(\hat{a}\left(\tau, \vec{q}_{3}\right) \hat{b}\left(\tau, \vec{q}_{4}\right)+\hat{b}\left(\tau, \vec{q}_{3}\right) \hat{a}\left(\tau, \vec{q}_{4}\right)\right)\right)\right]\right) .
\end{aligned}
$$




\section{REFERENCES}

[1] L.Lusanna, Int.J.Mod.Phys. A4 (1997) 645.

[2] L.Lusanna, 'The N-body Problem in Tetrad Gravity: a First Step towards the Unified Description of the Four Interactions', talk given at: XI Int.Conf. "Problems of Quantum Field Theory", Dubna 1998; III W.Fairbank Meeting and I ICRA Network Workshop "The Lense-Thirring Effect", Roma-Pescara 1998; 13th SIGRAV National Congress, Bari 1998; "Towards a Unified Description of the Four Interactions in Terms of DiracBergmann Observables", invited contribution to the book of the Indian National Science Academy for the International Mathematics Year 2000 AD (hep-th/9907081).

[3] S.Weinberg, "The Quantum Theory of Fields" (Cambridge Univ. Press, Cambridge, 1995).

[4] N.Nakanishi, 'Relative Time and Abnormal Solutions in the Theory of the BetheSalpeter Equation' and H.Sazdjian, 'Connection of Constraint Dynamics with the BetheSalpeter Equation', in "Extended Objects and Bound Systems", Karuizawa 1992, eds. O.Hara, S.Ishida and S.Naka (World Scientific, Singapore, 1993).

[5] D.Alba, L.Lusanna and M.Pauri, "Center of Mass, Rotational Kinematics and Multipolar Expansions for the Relativistic and Non-Relativistic N-Body Problem in the Rest-Frame Instant Form", in preparation.

[6] G.Longhi and M.Materassi, "Collective and Relative Variables for a Classical Real KleinGordon Field", Int.J.Mod.Phys. A14, 3387 (1999) (hep-th/9809024).

[7] G.Longhi and M.Materassi, "A Canonical Realization of the BMS Algebra", J.Math.Phys. 40, 480 (1999) (hep-th/9803128).

[8] D.Alba and L.Lusanna, Int.J.Mod.Phys A13, 2791 (1998) (hep-th/9705155).

[9] W.G.Dixon, J.Math.Phys. 8, 1591 (1967); "Extended Objects in General Relativity: Their Description and Motion" in "Isolated Gravitating Systems in General Relativity", ed.J.Ehlers (North-Holland, Amsterdam, 1979).

[10] M.Pauri and M.Prosperi, J.Math.Phys. 16, 1503 (1975).

[11] H.Feshbach and F.Villars, Rev.Mod.Phys. 30, 24 (1958).

[12] E.Corinaldesi and F.Strocchi, "Relativistic Wave Equations" (North-Holland, Amsterdam, 1963).

[13] F.Gross, "Relativistic Quantum Mechanics and Field Theory" (Wiley-Interscience, New York, 1993).

[14] W.Israel, "Covariant Fluid Mechanics anf Thermodynamics: an Introduction", in "Relativistic Fluid Dynamics", Noto 1987, eds. A.Anile and Y.Choquet-Bruhat, Lecture Notes in Math. 1385 (Springer, Berlin, 1989).

[15] K.S.Thorne, "The Theory of Gravitational Radiation: an Introductory Review" in 'Gravitational Radiation' eds. N.Deruelle and T.Piran, 1982 NATO-ASI School at Les Houches (North Holland, Amsterdam, 1983); Rev.Mod.Phys. 52, 299 (1980).

[16] W.Tulczyjew, Acta Phys.Polon. 18, 393 (1959).

[17] F.Pirani, Acta Phys.Polon. 15, 398 (1956).

[18] D.Bini, G.Gemelli and R.Ruffini, "Spinning Test Particles in General Relativity: Nongeodesic Motion in the Reissner-Nordström Spacetime" talk at the III W.Fairbank Meeting and I ICRA Network Workshop "The Lense-Thirring Effect", Roma-Pescara 1998.

[19] W.Beiglböck, Commun.Math.Phys. 5, 106 (1967). 
J.Ehlers and E.Rudolph, Gen.Rel.Grav. 8, 197 (1977).

R.Schattner, Gen.Rel.Grav. 10, 377 and 395 (1978).

[20] L.Lusanna, Int.J.Mod.Phys. A10, 3531, 3675 (1995).

[21] L.Lusanna and P.Valtancoli, Int.J.Mod.Phys. A12, 4769 (1997).

[22] C.Möller, "The Theory of Relativity" (Oxford Univ.Press, Oxford, 1957).

M.Pauri, "Invariant Localization and Mass-Spin Relations in the Hamiltonian Formulation of Classical Relativistic Dynamics", Parma Univ. preprint IFPR-T-019 (1971); in "Group Theoretical Methods in Physics" Lecture Notes in Physics No. 135, ed. K.B.Wolf (Springer, Berlin, 1980).

[23] Eds. W.P.Schleich and S.M.Barnett, "Quantum Phase and Phase Dependent Measurements", Physica Scripta vol. T48, (1993).

[24] K.Kuchar, J.Math.Phys. 17, 777, 792, 801 (1976).

[25] C.Lämmerzahl, J.Math.Phys. 34, 3918 (1993). 